
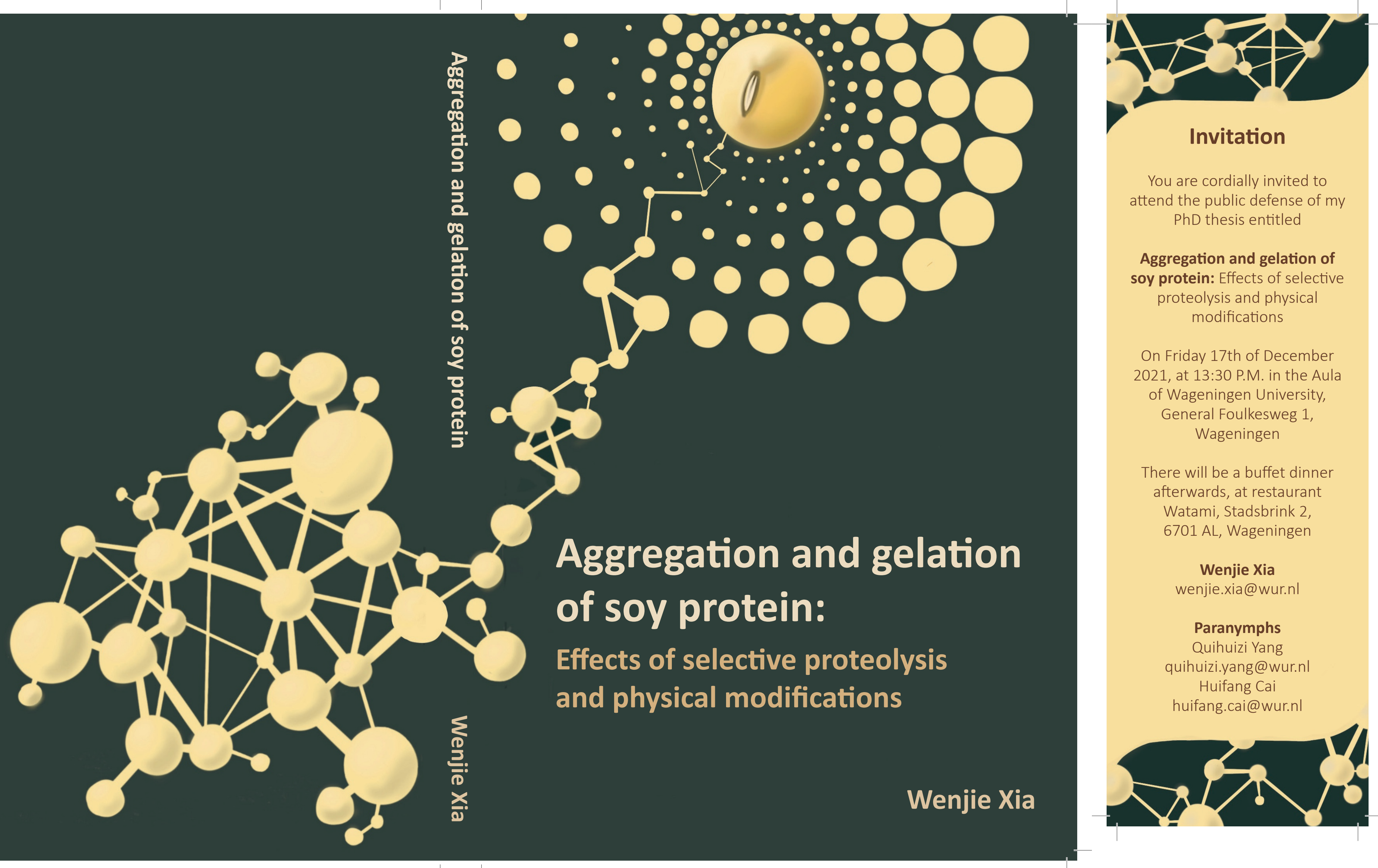


\section{Propositions}

1. The aggregation and gelation properties of soy protein can be tuned by selective proteolysis.

(this thesis)

2. Large amplitude oscillatory shear (LAOS) tests provide additional detailed information on protein hydrogel microstructures.

(this thesis)

3. The boost in soy protein-based foods does not drive deforestation in the Brazilian Amazon.

(Tyukavina et al. (2017) Types and rates of forest disturbance in Brazilian Legal Amazon, 2000-2013. Science Advances, 3(4), e1601047)

4. The development of meat analogues does not sufficiently consider different cuisine cultures.

5. Supervising students in your first PhD year is a burden, but in your last $\mathrm{PhD}$ year it is a blessing.

6. Life is fractal: great success often springs from rough times and repetitive work. (Mandelbrot, B. (2012). The fractalist: Memoir of a scientific maverick)

Propositions belonging to the thesis, entitled

Aggregation and gelation of soy protein: Effects of selective proteolysis and physical modifications

Wenjie Xia

Wageningen, 17 December 2021 


\section{Aggregation and gelation of soy protein:}

Effects of selective proteolysis and physical modifications

Wenjie Xia 


\section{Thesis committee}

\section{Promotor}

Dr L.M.C. Sagis

Associate professor, Physics and Physical Chemistry of Foods

Wageningen University \& Research

\section{Co-promotors}

Prof. Dr Siyi Pan

Professor, College of Food Science and Technology,

Huazhong Agricultural University, Wuhan, China

\section{Other members}

Prof. Dr Atze Jan van der Goot, Wageningen University \& Research

Dr LAM (Laurice) Pouvreau, Wageningen University \& Research

Dr Michel Mellema, Unilever, Wageningen, the Netherlands

Prof. Dr Anja Maria Wagemans, Technical University of Berlin, Germany

This research was conducted under the auspices of Graduate School VLAG

(Advanced Studies in Food Technology, Agrobiotechnology, Nutrition and Health Sciences). 


\title{
Aggregation and gelation of soy protein: \\ Effects of selective proteolysis and physical modifications
}

\author{
Wenjie Xia
}

\author{
Thesis \\ submitted in fulfilment of the requirements for the degree of doctor \\ at Wageningen University \& Research \\ by the authority of the Rector Magnificus, \\ Prof. Dr. A.P.J. Mol, \\ in the presence of the \\ Thesis Committee appointed by the Academic Board \\ to be defended in public \\ on Friday 17 December 2021 \\ at 1.30 p.m. in the Aula.
}


Wenjie Xia

Aggregation and gelation of soy protein: Effects of selective proteolysis and physical modifications

208 pages

PhD thesis, Wageningen University, Wageningen, the Netherlands (2021)

With references, with summary in English

ISBN: 978-94-6395-975-9

DOI: https://doi.org/10.18174/553455 


\section{Table of Contents}

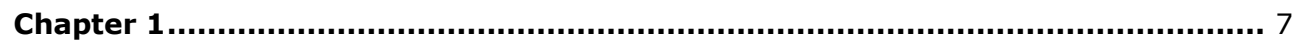

General introduction

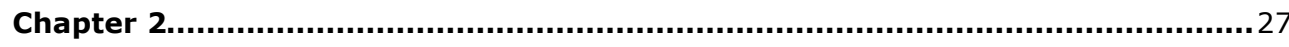

Formation of amyloid fibrils from soy protein hydrolysate: Effects of selective proteolysis on $\beta$-conglycinin

Chapter 3.

High-intensity ultrasound treatment on soy protein after selectively proteolyzing glycinin component: Physical, structural, and aggregation properties

Chapter 4 .

Linear and non-linear rheology of heat-set soy protein gels: Effects of selective proteolysis of $\beta$-conglycinin and glycinin

Chapter 5 .

Acid-induced gels from soy and whey protein thermally-induced mixed aggregates: Rheology and microstructure

Chapter 6.

General discussion

Summary

References 


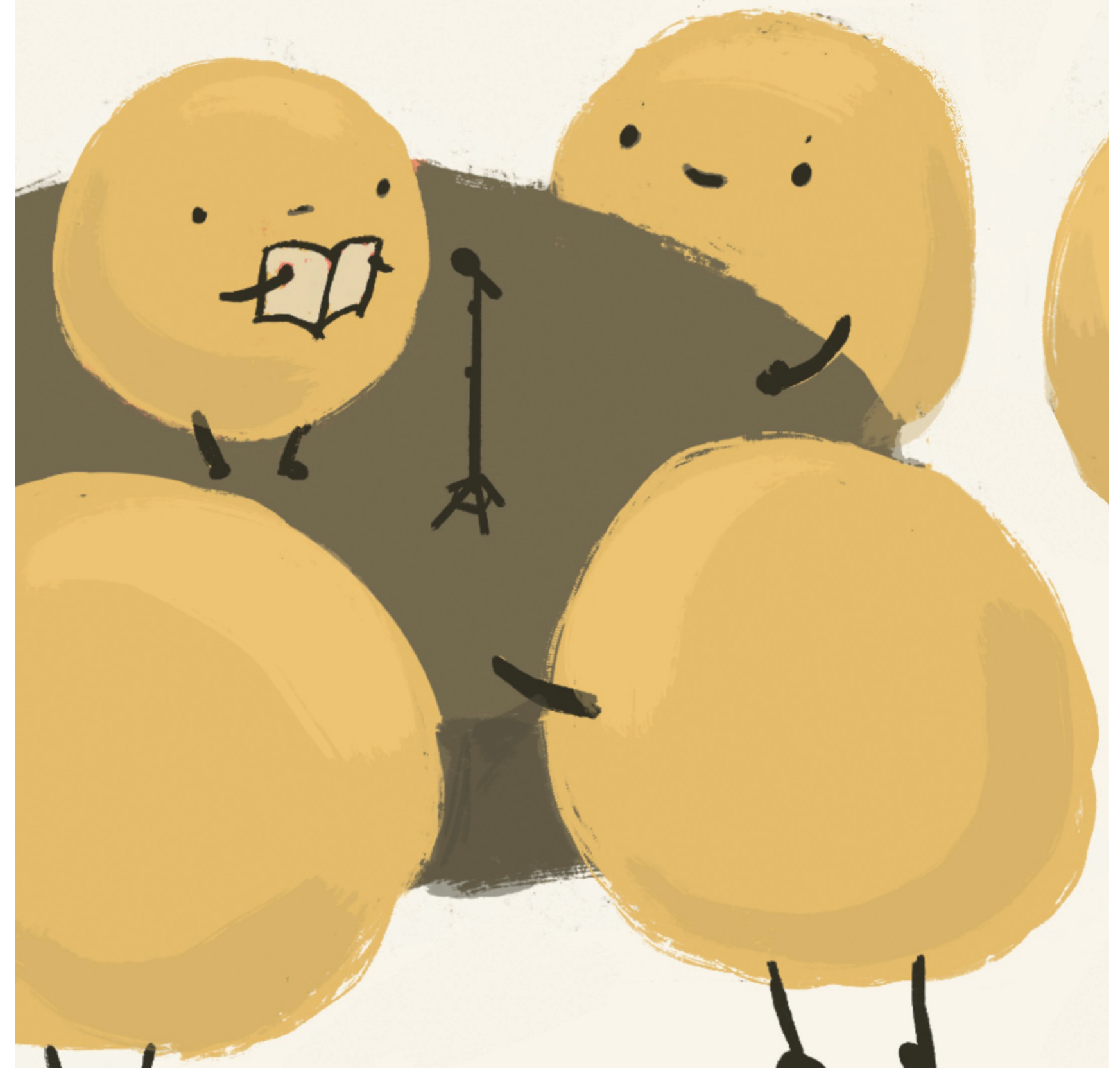




\section{Chapter 1}

General Introduction 


\subsection{Introduction}

Soybean or soya bean (Glycine max) is a species of legume that has been cultivated for thousands of years in China and other Asian countries, such as Japan and Korea. Since the 1960s, the cultivation and production of soybean have increased dramatically. The global production of soybean exceeded 334 million tons by 2019, led by countries such as the United States, Brazil, and Argentina (Fig. 1.1), which made soybean one of the most abundant protein resources in the world. Soy protein has been part of the daily diet for people in many countries, and various soy protein-based foods have been created such as soy milk, tofu (soybean curd), natto, and tempeh. In some Asian countries, these foods have played an indispensable role in their traditional cuisine and culture.
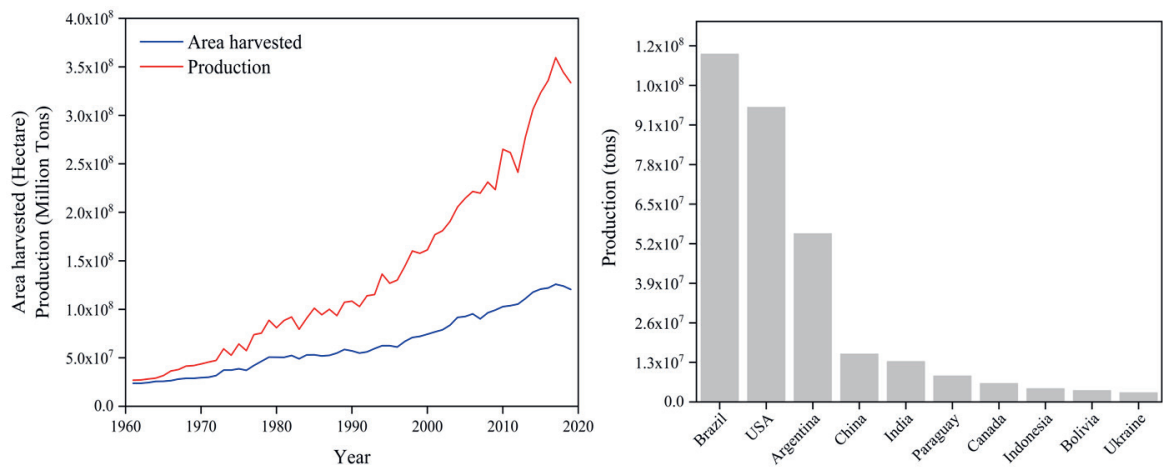

Figure 1.1. Global yield/ production quantities of soybean from 1961 to 2019 (A); Top 10 producers of soybean in 2019 (B). Data from FAOSTAT (The Food and Agriculture Organization Corporate Statistical Database).

Although there is a burgeoning interest in using soy protein as an ingredient in the food industry, the functional properties of soy protein still need to be improved before they are able to replace traditional animal-based proteins. The strategy to alter these functional properties, so-called protein modification, has been a research area of continued interest. 
To date, such modifications have been induced by chemical treatments (e.g., pH shifting and glycation/ Maillard reactions), physical treatments (e.g. heat treatment, ultrasonic treatment, and blending with other biopolymers), biological treatments (e.g. via enzymatic hydrolysis), and a combination of these treatments. A schematic overview of the protein modification methods is illustrated in Fig. 1.2. As the involvement of chemical reagents normally reduces consumers' acceptability of food products, food industries are shifting away from chemical modifications. Among the functional properties of soy protein, the aggregation and gelation properties are very important for their application, as these properties play an important role in the texture of many food products, such as tofu and meat analogues. This thesis contributes to the understanding of the aggregation and gelation properties of soy protein by investigating how these properties are affected by some emerging modification methods.

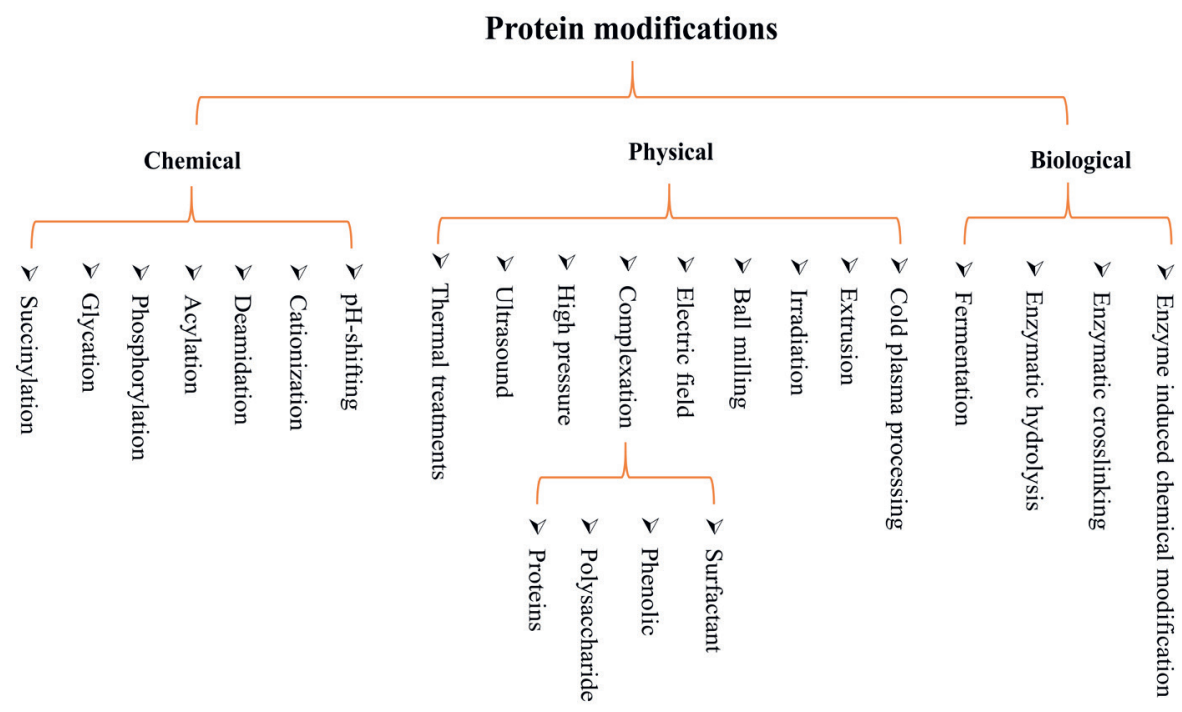

Figure 1.2. A schematic overview of the protein modification methods.

As an oil crop, soybean contains approximately $40 \%$ protein and $20 \%$ oil on an average dry matter base. After oil extraction, a large amount of defatted soy flour (protein content: $10 \sim 20 \%$ ) remains. From this defatted soy flour, soy protein ingredients with high purity can be prepared, such as soy protein concentrate (SPC, protein content: 55 60\%) and soy protein isolate (SPI, protein content: $>80 \%$ ), which are normally used in the food industry. Like many plant-based proteins, soy protein is a protein mixture that consists of many components. Based on the sedimentation coefficients, soy protein components can be 
classified into four protein categories i.e. $2 \mathrm{~S}, 7 \mathrm{~S}, 11 \mathrm{~S}$ and $15 \mathrm{~S}$, with more than $80 \% \beta$ conglycinin (7S) and glycinin (11S). The ratio of $\beta$-conglycinin/glycinin in soy protein depends on the soybean varieties as well as the cultivation environments. $\beta$-conglycinin is a trimeric glycoprotein with a molecular weight (MW) of $180 \sim 210 \mathrm{kDa}$ and an isoelectric point (IP) at pH 4.5 to 5.0. It consists of three subunits: a ( $67 \mathrm{kDa}), \mathrm{a}^{\prime}(\sim 71 \mathrm{kDa})$, and $\beta$ ( $50 \mathrm{kDa})$, associated by noncovalent interactions [2]. Glycinin in 11S form is a hexamer with an MW of $\sim 350 \mathrm{kDa}$ and an IP at pH 6.3 to 7.0. It consists of five different subunits, each subunit consists of an acidic polypeptide A (acidic pI) with a MW of 37-42 kDa and a basic polypeptide $B$ (basic pI) with a MW of $17-20 \mathrm{kDa}$, linked by a disulfide bond. $A B$ subunits are believed to associate into two hexagonal rings forming a hollow cylinder, by electrostatic interactions and hydrogen bonding, as shown in Fig. 1.3.
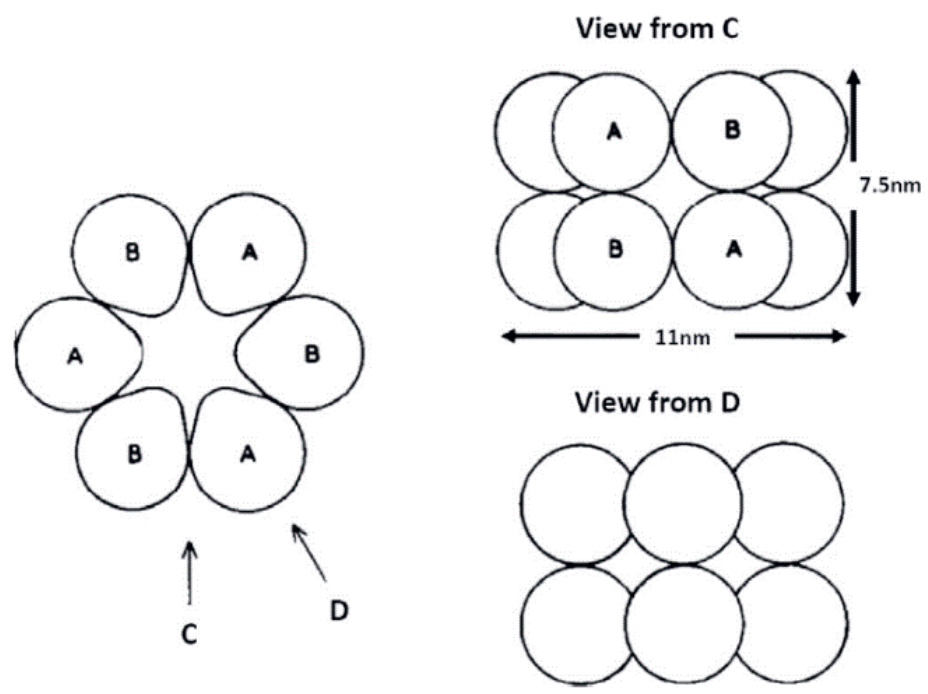

Figure 1.3. Schematic diagram of glycinin molecule consisting of acidic, A, and basic, B, subunits [3].

\subsection{Aggregation of soy protein}

\subsubsection{Random aggregates}

When globular proteins such as soy protein are denatured, their rigid conformational structures become looser, and convert into intermediate states (e.g. pre-molten globule, molten globule) or a totally unfolded state. Proteins in these denatured states tend to interact with each other, leading to a phenomenon known as protein aggregation. Despite many efforts, the aggregation of soy protein has not been understood thoroughly due to the complexity of this process and the fact that it is a mixed protein system. The dominant components of soy protein, $\beta$-conglycinin and glycinin possess different thermal aggregation 
behaviors due to different structures. Guo et al. compared the different thermal aggregation behaviors of $\beta$-conglycinin and glycinin at pH 7.0 (illustrated in Fig. 1.4) [2]. They found that $\beta$-conglycinin tended to form soluble aggregates via the consumption of monomers, while glycinin formed insoluble aggregates via further association between aggregates with high MW [2]. Soluble aggregates of $\beta$-conglycinin possessed a less compact conformation while insoluble aggregates of glycinin had a conformation of a denser core and a looser outer shell [2]. In addition, $\beta$-conglycinin could contribute to the solubilization of soy protein thermal aggregates, as heating in the presence of $\beta$-conglycinin restricted the self-assembly between the glycinin aggregates and recovered their solubility [2]. This was consistent with the findings of Damodaran et al. that adding purified $\beta$-conglycinin prevented thermal aggregation of glycinin and its isolated basic subunits, due to the formation of a soluble complex between the $\beta$-conglycinin and the basic subunits of glycinin [4].
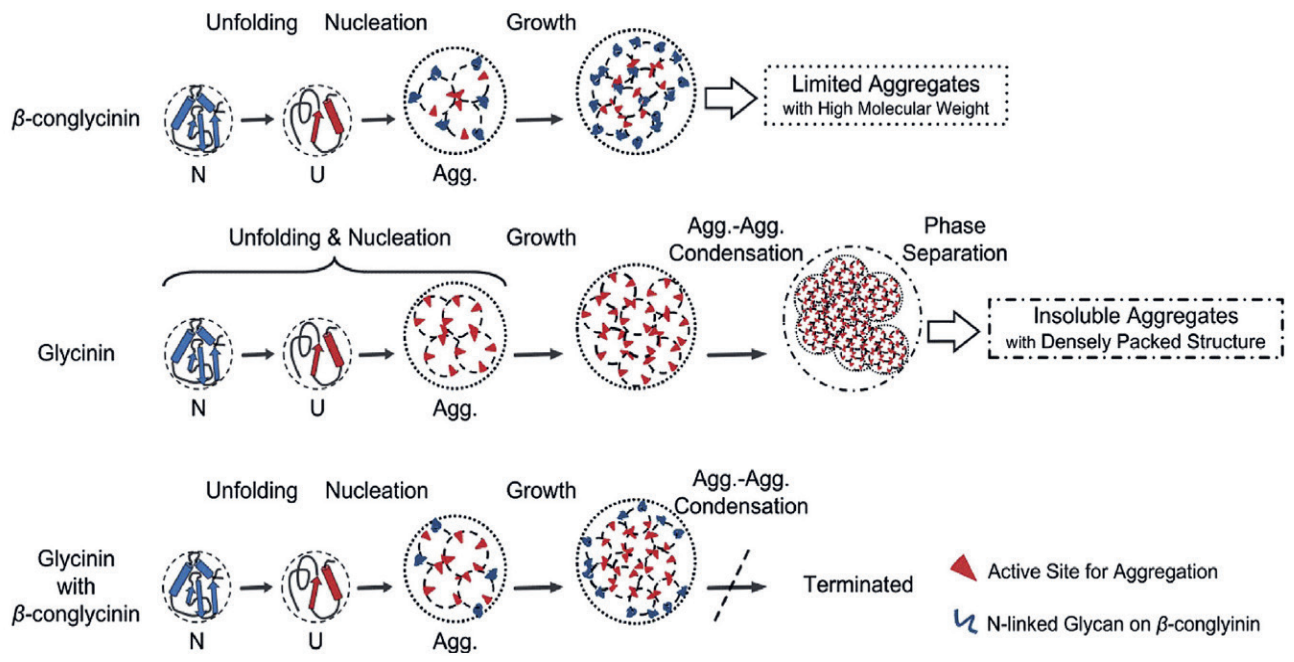

Figure 1.4. A schematic illustration of the thermal aggregation behavior of $\beta$-conglycinin and glycinin at pH 7.0. N: native state; U: unfolded state; Agg.: aggregates [2].

\subsubsection{Fibrillar aggregates}

Recently, the aggregation of proteins heated at low ionic strength and low $\mathrm{pH}$ has gained a lot of attention, as in this condition proteins have been found to form amyloid fibrils. Amyloid fibrils are ordered aggregates of peptides or proteins that are fibrillar in structure [5]. Nilsson (2004) proposed three criteria that define a protein aggregate as an amyloid fibril: 1) green birefringence upon staining with Congo Red, 2) fibrillar morphology, and 3) $\beta$-sheet secondary structure [5]. These proteinaceous aggregates with a typical cross- $\beta$ secondary structure, have a diameter of several nanometers, contour lengths up to a few micrometers, and an extremely high Young's modulus [5]. Although the interest originally arose from the 
clinical findings that amyloid fibrils are related to many diseases (e.g., Alzheimer's disease, type 2 diabetes mellitus, and Creutzfeldt-Jacob disease), amyloid fibrils have drawn attention from many food scientists because of their potential usage as thickening agents or texture modifiers. The current view is that amyloid fibrillation, as a special aggregation behavior, is a generic feature of almost all food proteins. Akkermans et al. reported that long and semiflexible fibrillar aggregates can be formed from soy protein isolate when heated at $85^{\circ} \mathrm{C}$ and $\mathrm{pH} 2.0$ [6]. They showed that the soy protein fibrils have a contour length of $\sim 1 \mu \mathrm{m}$, persistence length of $2.3 \mu \mathrm{m}$, and thickness of a few nanometers [6]. Tang and Wang (2010) also demonstrated the formation of amyloid-like fibrils when heating soy $\beta$ conglycinin and glycinin and their mixture $(1: 1)$ at $80^{\circ} \mathrm{C}$ and $\mathrm{pH} 2.0$, as all three criteria for amyloid fibrils proposed by Nilsson were satisfied [7]. More recently, Wang et al. revealed the effects of heating time $\left(85^{\circ} \mathrm{C}, \mathrm{pH} 2.0,0-24 \mathrm{~h}\right)$ and incubation times (0-7 days) on the formation and physicochemical properties of SPI amyloid fibrils, which showed that longer heating time enhanced the aggregation potential of protein hydrolysates while longer incubation time led to a slight increase of the particle size of fibrillar aggregates [8]. All findings indicated that the fibrillation of soy protein, like their aggregation in any other conditions, are affected by many intrinsic factors (e.g. protein composition, concentration, conformational structures) and extrinsic factors (e.g. heating time, incubation time, shear flow).

\subsection{Gelation of soy protein}

It is generally accepted that the gelation process is essential for the sensory and textural properties of many food products. Soy protein is the only plant-based protein that has been widely consumed in gel-based foods (e.g., tofu, yuba, and tofu pudding). It has also been added to other food products such as sausages and meat analogues because of its gelling ability. Gelation of soy protein often involves protein denaturation/unfolding, dissociation, association, and aggregation. The building blocks of protein gels can be particulate aggregate or strand aggregates. However, compared to the gelation processes of linear biomacromolecules such as polysaccharides, the understanding of the gelation process of soy protein is still limited because of its complexity.

\subsubsection{Heat-set gels}

Gelation of soy protein is most often induced by heat treatment. A mechanism for the network formation in the thermal gelation process of glycinin has been proposed (illustrated in Fig. 1.5): (1) Within a short time of heating (about $15 \mathrm{~s}$ ), glycinin molecules are moderately unfolded but still in a globular form, and six of these molecules are bound to a short strand (strand I); (2) Subsequently, strand I associates with itself to form a long 
straight strand (strand II); (3) Strand II associates with itself and/or strand I to form both branched and unbranched strands (strand III) [9]. The gel network could then form from these strand III units.

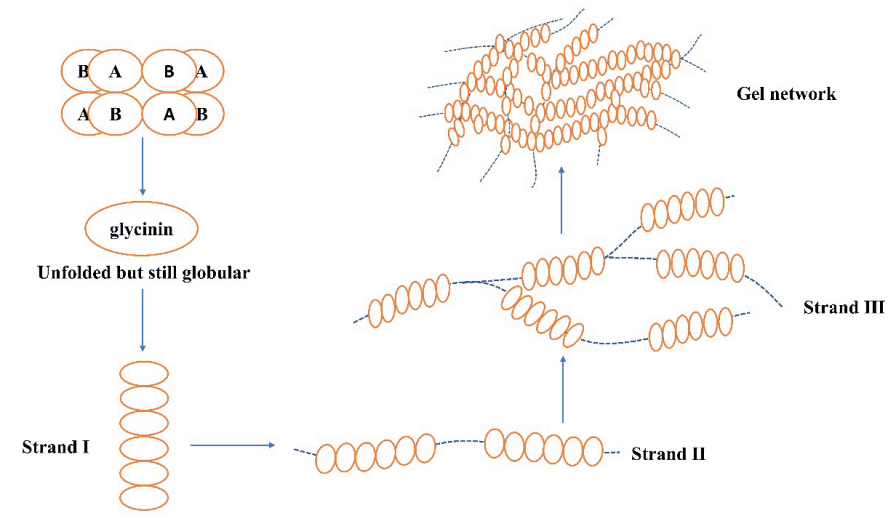

Figure 1.5. Thermal gelation mechanism of soy glycinin [9].

Heat-induced gelation process can be affected by many factors $(\mathrm{pH}$, temperature, salt concentration, etc.), and so are the final gel properties. For example, Renkema and colleagues found soy protein solutions gelled at a lower temperature at $\mathrm{pH} 3.8$ than at $\mathrm{pH}$ 7.6 [10]. SPI and soy glycinin were found to form coarse gels at pH 3.8 with a granulated, white appearance, while more fine-stranded gels were formed at $\mathrm{pH} 7.6$ with a smooth, slightly turbid appearance [11,12]. They also investigated the effects of different heating/cooling rates, heating times, and heating temperatures on the thermal gelation of SPI at $\mathrm{pH} 7.0$ [13]. Their results showed that the storage modulus $\left(\mathrm{G}^{\prime}\right)$, which is a measure of gel stiffness, increased with prolonged heating time but decreased with heating rate [13]. Higher heating temperature also led to a higher $\mathrm{G}^{\prime}$ as more proteins were thermally denatured, while no further increase of $\mathrm{G}^{\prime}$ was observed when the temperature was higher than $90^{\circ} \mathrm{C}$ [13]. The cooling step increased the $\mathrm{G}^{\prime}$ significantly, but this stiffening was found thermoreversible and unaffected by the cooling rates. Salt concentration also influenced the gelation [13]. It has been found that increased $\mathrm{NaCl}$ concentration slowed down the gelling rate and postponed the onset time of $\beta$-conglycinin gelation, which was related to the fact that the denaturation temperature of soy protein shifted to higher temperature with increasing ionic strength, as revealed by DSC measurements $[12,14,15]$. In addition, the influence of $\mathrm{NaCl}$ concentrations on the $\mathrm{G}^{\prime}$ of SPI gels was found to be $\mathrm{pH}$-dependent: the $\mathrm{G}^{\prime}$ was highest at $0,0.5$, and $0.2 \mathrm{M} \mathrm{NaCl}$ concentration when the $\mathrm{pH}$ was $3.8,5.2$, and 7.6, respectively [16]. Both the $\mathrm{pH}$ and salt concentration could affect the net charges of protein, which thus change the gel properties like transparency. Typically, at low salt concentration and $\mathrm{pH}$ values away from the isoelectric point of the protein, protein molecules maintain 
high net charges, and electrostatic repulsive forces between each other are relatively strong, which limits the formation of large aggregates and thus lowers the gel turbidity.

\subsubsection{Cold-set gels}

In contrast to the heat-induced gelation where the denaturation, aggregation and gelation are intertwined and occur simultaneously, soy protein gels can also be prepared by two separated steps: Firstly, native proteins are denatured and protein aggregates are formed by preheating a protein solution at a $\mathrm{pH}$ far away from the isoelectric point and a relatively low concentration. Secondly, by adding coagulants such as acidifiers and salts, the $\mathrm{pH}$ of the protein solution is lowered, the net charge and strength of the electrostatic repulsion between proteins are reduced, and salt bridges may also be formed, which all favor the formation of a space-filling network. The aggregates formed during preheating could be the structural units of cold-set gels since the molecular structures of the proteins in preheated solutions and formed gels were found to be similar [17]. Since the second step can be performed at ambient temperature, this type of protein gels is also known as cold-set gels. Cold-set gels have shown some superior properties compared to conventional heat-induced gels, such as higher transparency and lower critical gelation concentration. Furthermore, since heat-sensitive or volatile compounds, such as riboflavin and essential oil, can be added to the cooled protein suspension before the addition of coagulants, cold-set gels are highly desirable as an encapsulation and delivery system in not only the food sector but also the pharmaceutical industry $[18,19]$.

While cold-set gels have been widely produced with whey proteins, the number of studies on soy protein cold-set gels are still limited so far. Ais and colleagues proved that preheated SPI solutions can form cold-set gels by adding $\mathrm{Ca}^{2+}$, and gels with different opacity, waterholding capacity and rheological properties can be obtained by varying the concentration of SPI and $\mathrm{CaCl}_{2}$ [20]. As an acidic coagulant, Glucono- $\delta$-lactone (GDL) is also widely used to prepare cold-set soy protein gels in the food industry such as lactone Tofu. It continuously releases protons in the protein solution and thus lowers the $\mathrm{pH}$ toward the isoelectric point of the protein, and reduces the electrostatic repulsion, bringing the protein aggregates closer together to form progressively a three-dimensional network. The GDL-induced gelation can be affected by GDL/protein ratio and the preheating/gelation conditions (temperature, time, ionic strength, etc.). For example, like for heat-set gels, Campbell et al. found the gel strength and hardness of GDL-induced SPI gels increased with protein concentration [21]. At the same protein concentration, a higher GDL concentration led to a lower final $\mathrm{pH}$ of the gel, of which the strength and hardness peaked at pH 4.5 [21]. Schuldt et al. reported that under the same addition of GDL, a higher temperature can accelerate the release of gluconic acid, and thus the acidification rate, leading to an earlier gelation onset [22]. A faster 
acidification rate also resulted in a weaker gel because less time was available for the formation and rearrangement of the bonds between proteins [22]. In addition to the gelation step, the conditions of the preheating step can also regulate the properties of acid-induced SPI gels. Recently, it has been found that a higher SPI concentration during the preheating step could increase the particle size and surface charges of soluble aggregates, and decrease their surface hydrophobicity, which resulted in a more uniform gel network with improved hardness, elasticity, water holding capacity and toughness [23].

\subsection{Food rheology}

As a study of the flow and deformation of materials, rheology has been applied in food researches for decades, with the aim to monitor the texture development of food products and predict their sensory properties. Rheological tests can be divided into several types, including compressive, rotational, and oscillatory shear tests. Among them, oscillatory shear tests prove to be useful to study semisolid materials such as food biopolymer gels. Depending on the deformation amplitude, oscillatory shear tests can be further divided into two types, i.e. small amplitude oscillatory shear (SAOS) and large amplitude oscillatory shear (LAOS) tests. SAOS tests subject gels to sufficiently small strain amplitudes that will not disrupt the gel microstructure. SAOS tests characterize the rheological properties of gels in the linear viscoelastic (LVE) regime where the shear moduli are independent of the magnitude of the applied strain or strain rate. In the LAOS test, the strain amplitude is increased to a level where the gel microstructure is affected, and the mechanical response of gels falls in the non-linear viscoelastic (NLVE) regime where the shear modules become a function of the strain and strain rate.

So far, SAOS tests are almost routinely applied in the studies of food protein gels. For example, the evolution of storage modulus $\left(G^{\prime}\right)$ and loss modulus $\left(G^{\prime \prime}\right)$ of soy protein solutions can be recorded during a complete temperature cycle (heating and cooling), which simulates the thermal gelation process [13]. The $\mathrm{G}^{\prime}$ represents the elastic response while the $G^{\prime \prime}$ measures the viscous dissipation per cycle of the sinusoidal deformation of a gel. Typically, the point when $\mathrm{G}^{\prime}$ of the protein solution surpasses the $\mathrm{G}^{\prime \prime}$ is defined as the onset of gelation (i.e. gelling point). Some studies also take the point where $\mathrm{G}^{\prime}$ starts to increase significantly as the gelling point. Various geometries can be used for SAOS tests, such as parallel plate, cone-plate, and concentric cylinder. The selection of geometry depends on the nature of testing samples and research purposes. In this thesis, we used a concentric cylinder since it could measure a large sample volume and generates a homogeneous strain field, which is beneficial for performing more sensitive measurements such as the LAOS tests we performed subsequently. Besides, this geometry could better prevent water evaporation 
during thermal gelation, since the shear gap is covered from above by a thick layer of excess samples and a solvent trap.

\subsubsection{LAOS tests}

In the production (e.g., mixing and transportation) or in the digestion process (e.g., chewing and swallowing), food materials are inevitably subjected to large strains and stresses. Therefore, the results obtained from LAOS tests can be better linked to the actual processing and sensory properties of food materials than SAOS tests. Although this has been widely recognized, only a few food studies have applied LAOS tests so far. Performing LAOS tests itself is not difficult, as neither a special rheometer nor measuring geometry is needed to handle this test. Beyond the LVE regime, the stress signal in LAOS tests becomes a distorted waveform instead of the perfect sine or cosine waveform obtained in SAOS tests. The challenges lie in how to analyze this distorted raw signal and how to extract some parameters with physical meaning that can be robustly linked with structural features and sensory attributes. For this reason, linking the results of LAOS tests on soy protein gels to their microstructures forms an integral part of this thesis.

Till now, two main strategies for systematically interpreting LAOS data has been proposed: One strategy is output wave data decomposition, often accompanied by the analysis of various harmonics in the wave data to extract parameters that describe NLVE behavior. In this strategy, Fourier transforms (FTs) are often used to convert the output waveform to a Fourier series, and an FT of a LAOS wave normally shows multiple peaks at odd harmonics $(n=1,3,5, \ldots)$. The number and intensity of the peaks above the noise level are thus used as a fingerprint for LAOS material behaviors. Although this Fourier transform rheology can be useful to differentiate materials with similar SAOS behaviors, it is difficult to interpret the obtained harmonics data, and connect it to the microstructural changes.

This thesis adapted another strategy that focuses on the plots of viscoelastic moduli vs. strain or stress. The patterns in these plots are signatures of certain types of rheological behaviors and linked to the microstructural changes in materials. As shown in Fig. 1.6, Hyun et al. classified four main types of LAOS behavior for complex fluids, based on how their viscoelastic moduli changed beyond the linear viscoelastic region (LVR): type I, strain thinning ( $G^{\prime}, G^{\prime \prime}$ both smoothly decreasing with increasing strain); type II, strain hardening ( $G^{\prime}, G^{\prime \prime}$ both increasing); type III, weak strain overshoot ( $G^{\prime}$ decreasing, $G^{\prime \prime}$ first increasing followed by decreasing); type IV, strong strain overshoot ( $G^{\prime}, G^{\prime \prime}$ first both increasing followed by decreasing) [24]. Like Fourier transformation, this qualitative analysis can differentiate materials with similar SAOS behaviors. However, this method only works for 
homogeneous fluids and semisolids, and the microstructural features behind the observed behaviors are not completely evident.

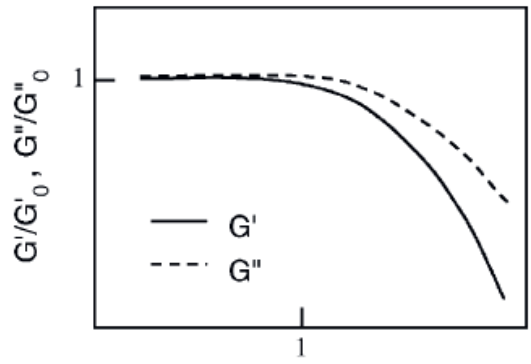

(a)

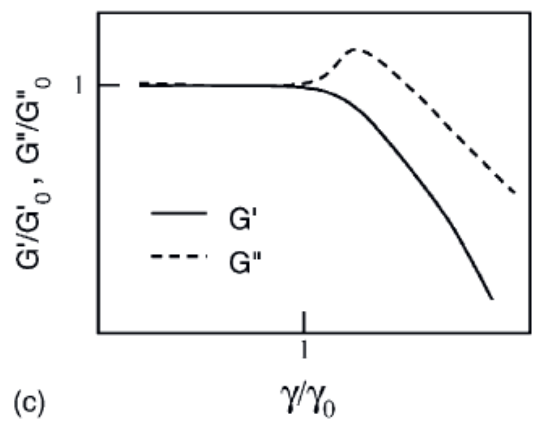

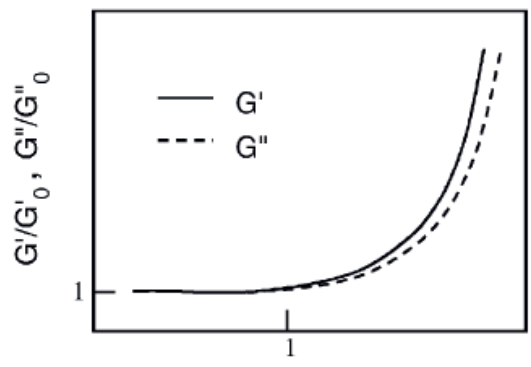

(b)

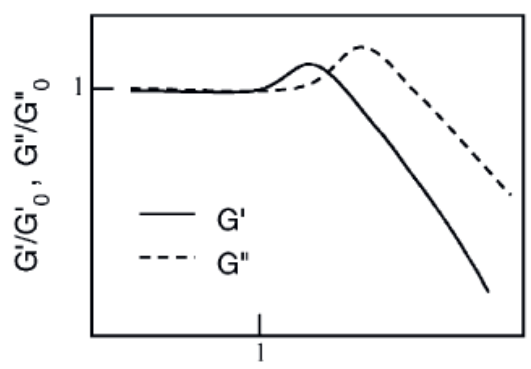

(d)

Figure 1.6. Four main types of LAOS behavior for complex fluids. (a) strain thinning; (b) strain hardening; (c) weak strain overshoot; (d) strong strain overshoot [24].

Another method that is extensively used in this thesis, is based on Lissajous-Bowditch plots (often called Lissajous plots), which has been extensively discussed by Ewoldt et al. [25]. Lissajous plots are the plots of intracycle stress versus intracycle strain or intracycle strain rate at a specific amplitude and frequency (Fig. 1.7). These plots can be obtained at different strains and frequencies, and then arranged in a Pipkin diagram, and thus another kind of rheological fingerprint can be established for a particular material. Based on the shape of Lissajous plots, the relative extent and type of non-linear viscoelastic (NLVE) behavior can be qualitatively evaluated. If the Lissajous plots are normalized, semiquantitative analysis is possible by calculating and comparing the enclosed area of loops. Ewoldt et al. defined two new viscoelastic moduli and two new instantaneous viscosities which are rheological parameters that can be obtained from Lissajous plots (Fig. 1.7): $\mathrm{G}^{\prime} \mathrm{L}$, the large-strain elastic modulus $(\mathrm{Pa})$, which is the slope of the secant line at maximum intracycle strain; $\mathrm{G}^{\prime}{ }_{\mathrm{M}}$, the minimum-strain elastic modulus ( $\mathrm{Pa}$ ), is the slope of the tangent line at zero intracycle strain [25]. Similarly, in the viscous Lissajous-Bowditch plot, $\eta^{\prime}\llcorner$, the large-rate instantaneous viscosity (Pa.s), and is the slope of the secant line at maximum strain rate; $\eta^{\prime} \mathrm{m}$, the 
minimum-rate viscosity (Pa.s), which is the slope of the tangent line at minimum strain rate [25]. These four parameters can be converted into two unitless indices, i.e. the strainstiffening ratio (S-factor) and shear-thickening ratio ( $T$-factor):

$$
\begin{gathered}
S=\frac{G_{L}^{\prime}-G_{M}^{\prime}}{G_{L}^{\prime}}\left\{\begin{array}{l}
>0 \\
=0 \\
<0
\end{array}\right. \\
T=\frac{\eta_{L}^{\prime}-\eta_{M}^{\prime}}{\eta_{L}^{\prime}}\left\{\begin{array}{l}
>0 \\
=0 \\
<0
\end{array}\right.
\end{gathered}
$$

Strain stiffening linear elastic Strain softening

Shear thickening linear viscous Shear thinning

In this way, quantitative analysis of strain- and shear-dependent NLVE behaviors can also be achieved based on Lissajous plots.
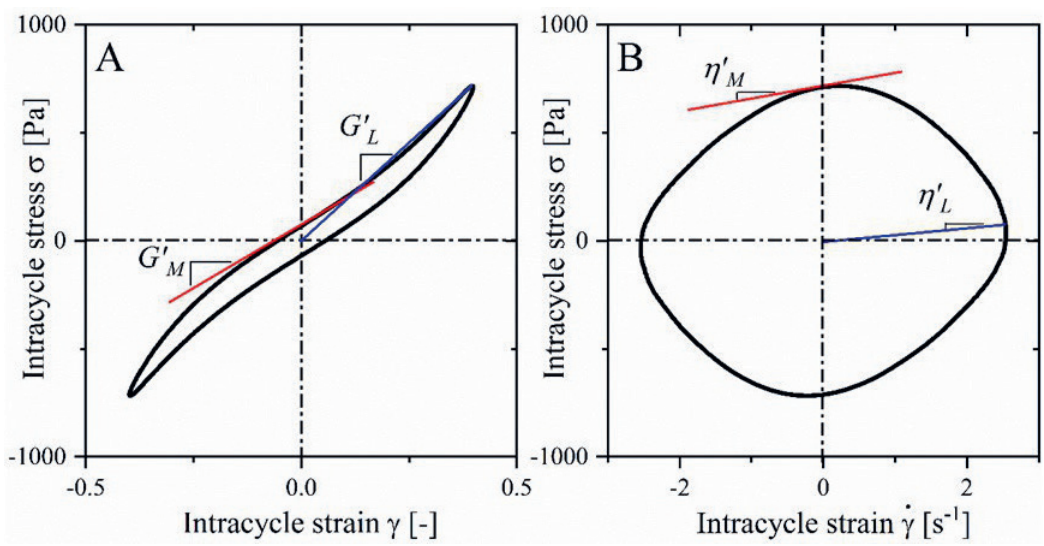

Figure 1.7. A schematic of elastic moduli (A) in an elastic Lissajous plot, and dynamic viscosities (B) in a viscous Lissajous plot.

In this thesis, fractal scaling theory was used to link the rheological properties to gel microstructures. Shih et al. developed a fractal scaling model relating the rheological properties ( $\mathrm{G}^{\prime}$ and linear critical strain $y_{0}$ ) to the particle volume fraction $\varphi$ for colloid gels, by considering the gel network as a collection of fractal flocs which are closely packed throughout the sample [26]. A schematic representation of colloidal gels is displayed in Fig. 1.8 , where the details of colloid flocs (aggregates) are not shown and they will look more complicated in reality. Depending on the relative strength of inter-floc and intra-floc links, gels are classified either as strong-link (inter-floc links stronger than intra-floc links) or weak-link (inter-floc links weaker than intra-floc links). The increase of $\mathrm{G}^{\prime}$ with increasing $\varphi$ is slower in the weak-link regime than that in the strong-link regime. Furthermore, yo increases with increasing $\varphi$ in the weak-link regime, while $y_{0}$ shows a decreasing trend with 
increasing $\varphi$ in the strong-link regime. Different scaling relations hold in these two regimes:

Weak-link regime $\left\{\begin{array}{l}G^{\prime} \sim \varphi^{(d-2) /\left(d-D_{f}\right)} \\ \gamma_{0} \sim \varphi^{1 /\left(d-D_{f}\right)}\end{array}\right.$ Strong-link regime $\left\{\begin{array}{l}G^{\prime} \sim \varphi^{(d+x) /\left(d-D_{f}\right)} \\ \gamma_{0} \sim \varphi^{-(1+x) /\left(d-D_{f}\right)}\end{array}\right.$

where $d$ is the Euclidean dimension (for gels this is 3 ), $D_{f}$ is the fractal dimension of the flocs, and $x$ is the backbone fractal dimension of the flocs or the tortuosity of the network (ranging from 1 to 1.3 for colloidal gels). The advantage of this model is that it can extract structural information of the colloid gels, such as the fractal dimension of the flocs, simply from rheological measurements. A disadvantage is that the strong-link and weak-link regimes in this model only represent two extreme situations, and it does not describe the intermediate regime where both the inter- and intra-floc links contribute to the overall elasticity of gels.

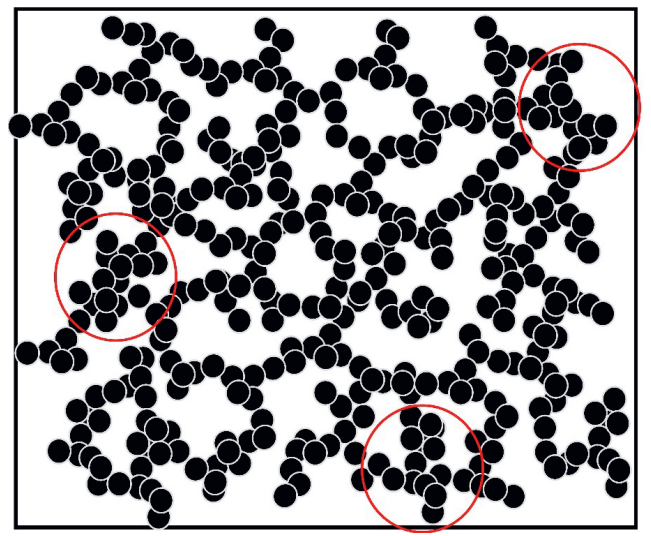

To make Shih's model more generally applicable, Wu and Morbidelli further developed this model by introducing a new parameter, $a$ (ranging from 0 to 1 ), which represents the relative importance of the elastic contribution from inter- and intra-floc links, and allows one to identify the different types of gelation regimes prevailing in the system [27]. The scaling model can be described as follows: 


$$
\begin{aligned}
& G^{\prime} \sim \varphi^{\beta /\left(d-D_{f}\right)} \\
& \gamma_{0} \sim \varphi^{(d-\beta-1) /\left(d-D_{f}\right)} \\
& \beta=(d-2)+(2+x)(1-\alpha)
\end{aligned}
$$

\subsection{Protein modification}

\subsubsection{Enzymatic proteolysis}

Enzymatic proteolysis converts protein into peptides of various sizes and free amino acids, which is a bioreaction that is comprised of the cleavage of the covalent peptide bond between the amino and carboxyl groups of two adjacent amino acids, catalyzed by a peptidase [28]. Compared to chemical hydrolysis, enzymatic hydrolysis not only avoids exogenous chemicals and preserves/improves the nutritive values of soy proteins to a larger extent, but also provides mild and more controllable processes by selecting different enzymes and reaction conditions. As an important concept and parameter, the degree of hydrolysis (DH) has been used to control the hydrolysis process and obtain reproducible hydrolysates. $\mathrm{DH}$ is normally defined as the percentage of peptide bonds cleaved but it also has been defined as the amount of nitrogen soluble in trichloroacetic acid after hydrolysis [29]. To measure the $\mathrm{DH}$, the $\mathrm{pH}$-stat method is often used, as it allows one to monitor the $\mathrm{DH}$ of the system in realtime. Depending on the $\mathrm{pH}$ of the medium, the carboxyl group and amino groups generated by proteolysis may lead to the release or consumption of protons, which results in $\mathrm{pH}$ changes. In response, $\mathrm{pH}$-stat devices automatically add acid or base to keep the $\mathrm{pH}$ unchanged during proteolysis, so the titration rate is proportional to the reaction rate, and the quantity of titrant added can be converted into the $\mathrm{DH}$ [30]. Other methods such as ophthaldialdehyde (OPA) and trinitrobenzenesulfonic acid (TNBS) methods can also measure the $\mathrm{DH}$, via the quantification of free amino groups. When the desired $\mathrm{DH}$ is reached, the proteolysis reaction is terminated by inactivating the enzyme via heating, $\mathrm{pH}$ shifting, or adding protease inactivators such as antipain. 
As DH only represents the proportion of cleaved peptide bonds after proteolysis, when the substrate is a multicomponent protein mixture like soy protein, it is hard to control which component of the protein is hydrolyzed. To overcome this problem, a novel method called selective proteolysis has been explored in this thesis. The principle of this method was based on the different denaturation conditions of soy protein components as well as the characteristics of different enzymes [31]. According to the results of differential scanning calorimetry (DSC) measurements (Fig. 1.9), $\beta$-conglycinin of native SPI starts to denature at around $70^{\circ} \mathrm{C}$ at pH 7.0, which is approximately $15^{\circ} \mathrm{C}$ lower than for glycinin. When native SPI is subjected to a temperature of $75^{\circ} \mathrm{C}$ at pH 7.0, $\beta$-conglycinin will be denatured to a much higher extent than glycinin in a certain time range. In contrast, it has been shown that glycinin is more preferentially denatured at $\mathrm{pH} 2.0$, compared to $\beta$-conglycinin. As a result, $\beta$-conglycinin and glycinin can be preferentially denatured at these two different reaction conditions, and the denatured component can be much more accessible to the enzyme than the other undenatured one. Selective denaturation is only a precondition and taking advantage of different features of enzymes is also important. Pepsin was used in the selective proteolysis of glycinin because it shows specificity to glycinin and has the highest potency in acidic conditions. Papain was used in the selective proteolysis of $\beta$-conglycinin because it is one of the few heat-resistant enzymes which has optimal activity at high temperatures. In this way, selectively proteolysis of $\beta$-conglycinin and glycinin of soy protein can be finally achieved.

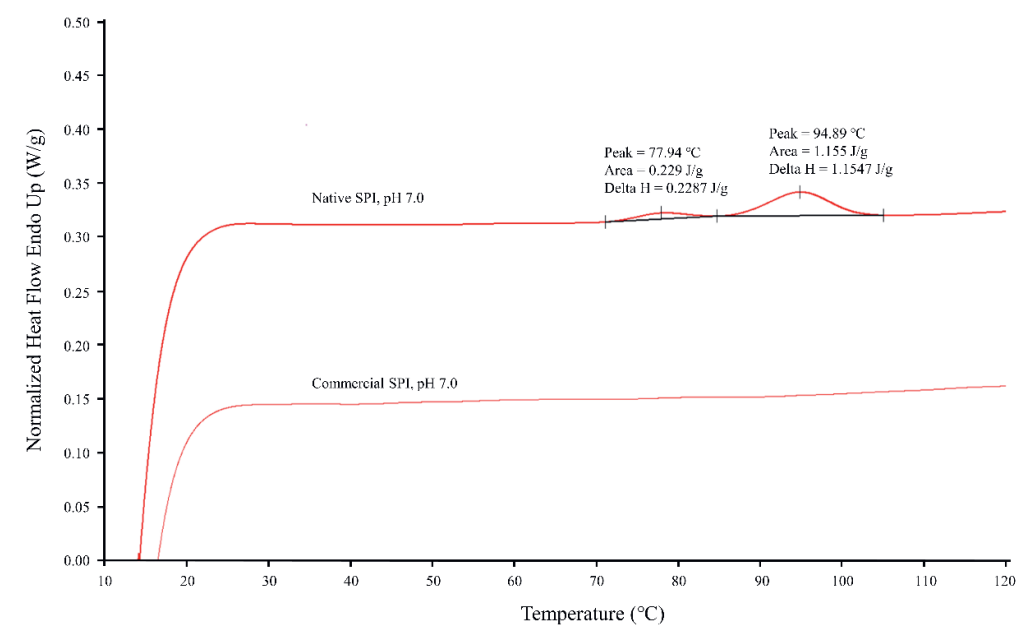




\subsubsection{Physical modification}

Physical modification normally involves the changes in the environmental conditions (e.g. temperature, humidity, the coexistence of other biomacromolecules) or the expose of proteins to a certain force field (e.g. pressure, ultrasound and electric). Heat treatment is one of the most widely used processing techniques for modifying soy protein. Controlled application of heat can be used to modify the structure of soy protein and consequently to improve some functional properties. Campbell et al. reported that preheated SPI showed a higher gel strength after acid-induced gelation than their unheated counterparts [21]. This increase in gel strength was recently proved to be more significant when preheating SPI at a higher concentration $(60 \mathrm{mg} / \mathrm{mL})[23]$.

Ultrasound is a form of high-frequency sound that is beyond the threshold of human hearing ( $>16 \mathrm{kHz}$ ). According to the frequency and power level, ultrasound can be divided into lowintensity ultrasound (LIU) (power $<1 \mathrm{~W} / \mathrm{cm}^{2}, 100 \mathrm{kHz}$ to $1 \mathrm{MHz}$ ) and high-intensity ultrasound (HIU) (power 10 to $1,000 \mathrm{~W} / \mathrm{cm}^{2}, 16$ to $100 \mathrm{kHz}$ ). The former is commonly used for the analytical evaluation of the physicochemical properties of foods, while the latter has been used for protein modifications recently [32]. HIU modifies the protein functionality mainly through the cavitation phenomenon produced by the ultrasonic sonotrode. When the HIU pass through the medium (where protein is dispersed), it causes compressions and rarefactions of the medium, leading to the formation of microscopically small voids. These voids become gas bubbles that rapidly grow in extension phases and shrink in compression phases until they implode. The implosion of bubbles on the microscale can generate localized high pressure, temperature, and hydrodynamic shear forces, which can change the molecular structures of proteins and thus their functionalities. A schematic of the HIU treatment used in this thesis (Chapter $\mathbf{3}$ ) and the cavitation phenomenon is presented in Fig. 1.10. 


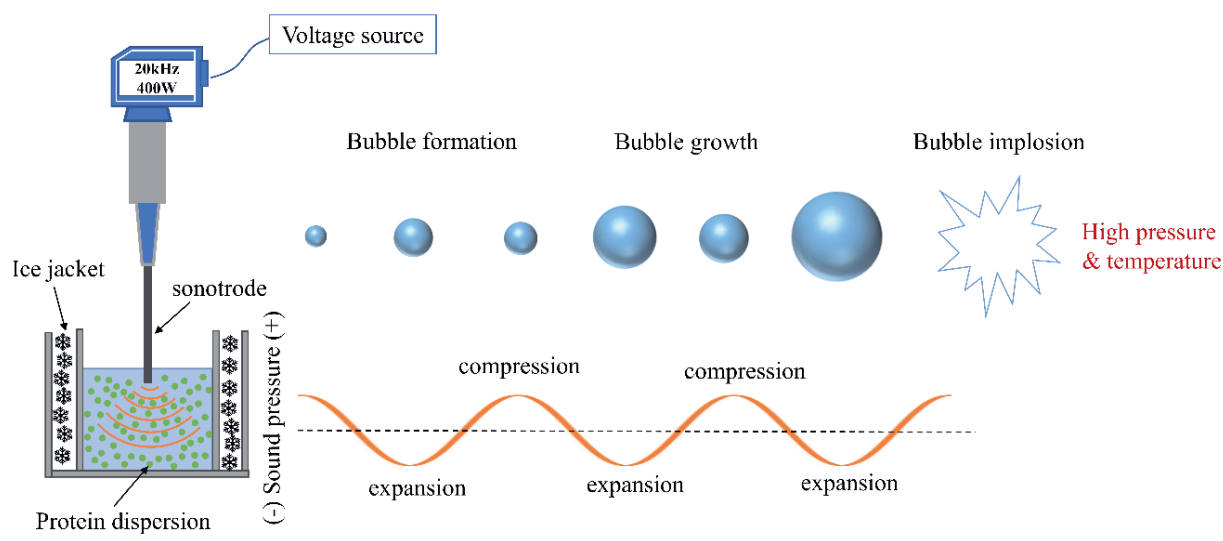

\subsection{Research aim and outline of this thesis}

As a benchmark for plant-based proteins, the functional properties of soy protein and its modifications are research areas of long-lasting interest in the food science field. This thesis aims to contribute to the understanding of the aggregation and gelation properties of soy protein by revealing how these properties are affected by some emerging modification methods. Since food protein hydrogels are semisolid material with viscoelastic properties, a comprehensive rheology study is a prerequisite for their application, and an important part of this thesis. In Chapter 2, we applied a novel method called selective proteolysis to selectively hydrolyzing the $\beta$-conglycinin component of native soy protein isolate (SPI), and thus obtained a hydrolysate named degraded $\beta$-conglycinin hydrolysate (DBH). After this, we compared the thermal aggregation behavior of $\mathrm{D} \beta \mathrm{H}$ and unmodified SPI at acid condition $(\mathrm{pH} \mathrm{2.0)}$ and low ionic strength $(20 \mathrm{mmol} / \mathrm{L})$, as under those conditions the protein was expected to self-assemble into amyloid fibrils. In Chapter 3, we applied selective proteolysis to selectively hydrolyzing the glycinin component of SPI, and obtained another type of hydrolysate named degraded glycinin hydrolysate (DGH). After this, we compared the aggregation behavior of DGH and SPI after exposure to a high-intensity ultrasound field (20 $\mathrm{kHz}$ at $400 \mathrm{~W}, 0,5,20$, and $40 \mathrm{~min}$ ). The hydrolyzing approaches used in the above Chapters were both applied in Chapter 4, where we prepared DBH and DGH at similar DH levels and compared the thermal aggregation and gelation behavior of these hydrolysates with SPI at $\mathrm{pH}$ 7.0. In addition, the roles of the peptides in these hydrolysates were considered by studying dialyzed hydrolysates at the same conditions. To systematically characterize the thermal gelation properties of SPI and selective hydrolysates, small amplitude oscillatory shear tests (SAOS), large amplitude oscillatory shear tests (LAOS), confocal laser scanning 
microscopy (CLSM), and scanning electron microscopy (SEM) were applied. The relationship between rheological properties and gel microstructures was examined with the help of fractal scaling theory. In Chapter 5, we investigated the thermal aggregation behavior $(\mathrm{pH} \mathrm{7.0)}$ of SPI in the presence of a traditional animal-based protein, i.e. whey protein isolate (WPI). The properties of mixed-aggregates formed by SPI and WPI were evaluated and these aggregates were further studied in terms of their acid-induced gelation properties. SAOS, LAOS, microscopy, and fractal scaling theory were applied on these cold-set gels to explore how their rheological and microstructural properties were affected by protein concentrations and SPI: WPI ratios. In Chapter 6, we present a general discussion based on the main findings of Chapter 2-5, with the aim to shed light on 1) the similarities and differences between different modifications, and 2) the interplay between modification, rheological properties, and microstructure. Finally, an outlook and recommendations for future studies are provided based on our reflections on the results in the current thesis. 


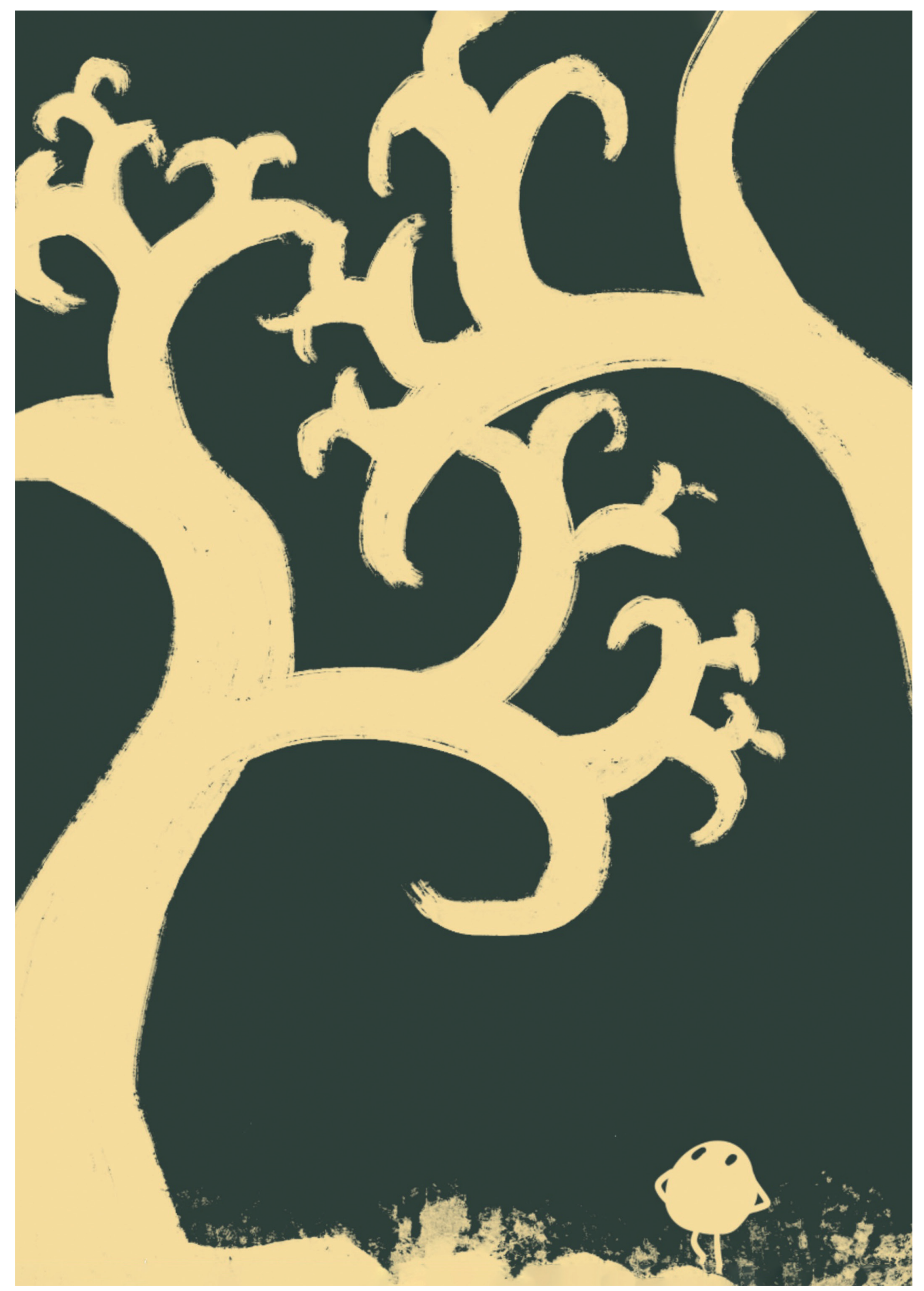




\section{Chapter 2}

\section{Formation of amyloid fibrils from soy protein hydrolysate: Effects of selective proteolysis on $\beta$-conglycinin}

Published as:

W. Xia, H. Zhang, J. Chen, H. Hu, F. Rasulov, D. Bi, X. Huang, S. Pan, Formation of amyloid fibrils from soy protein hydrolysate: Effects of selective proteolysis on $\beta$-conglycinin, Food Research International, 100 (2017) 268-276. doi:10.1016/j.foodres.2017.08.059. 


\section{Abstract}

Soy protein subjected to selective proteolysis on $\beta$-conglycinin (referred to as $\mathbf{D} \boldsymbol{\beta} \mathbf{H}$, contrast group) and a control soy protein isolate sample without addition of protease (referred to as CSPI, blank group) were adopted as experimental samples. By employing the "subtraction" mode of logical thinking, we aimed to compare the differences between CSPI and DBH on fibrillation at $\mathrm{pH} 2.0$ with heating at $95^{\circ} \mathrm{C}$. The results showed when heated for $60 \mathrm{~min}$, CSPI tended to form short worm-like fibrils while $D \beta H$ formed long semiflexible fibrils. When the heating time was prolonged to $360 \mathrm{~min}$, the fibrils formed from both exhibited clusters. Whereas when heated for $720 \mathrm{~min}$, no fibrillar aggregates appeared from them. This study would help explore the effects of $\beta$-conglycinin on the fibril formation of soy protein isolate in a new way. 


\subsection{Introduction}

Soybean is one of the major oil crops and the most economical protein source worldwide, as well as an important strategic material in world trade. Soy protein isolate (SPI) mainly consists of $\beta$-conglycinin and glycinin [33]. $\beta$-Conglycinin and glycinin dramatically influence the functional characteristics of soy protein and are the important intrinsic factors affecting the aggregation behavior of soy protein. Many previous researches on soy protein components (i.e., $\beta$-conglycinin and glycinin) basically followed a kind of similar route: first, the soy protein components were separated and purified from defatted soybean flour respectively according to the conventional method; and then, further researches on their functional characteristics under different physicochemical environments were taken [10,3437]. However, the results obtained from these researches could hardly effectively elucidate the role of individual soy protein isolate components (i.e., $\beta$-conglycinin and globulin) in the overall and interactive system of soy protein isolate.

So far, a few studies indicated that selective proteolysis could have been successfully employed in soy protein [38-40]. Based on different denaturation properties between $\beta$ conglycinin and glycinin, selective proteolysis technology could specifically and exclusively decompose one of them by selecting appropriate protease and reaction conditions $[31,39]$.

Fibrillar aggregates of food proteins have recently attracted increasing attention in the food science field owing to their potential applications (thickening, gelling ingredients, weightefficient structurants, and so on) in food products [7,41-44]. In the present study, the selective proteolysis technique was performed to obtain a kind of soy protein hydrolysate which was selectively degraded on $\beta$-conglycinin (referred to as $\mathbf{D} \boldsymbol{\beta} \mathbf{H}$, contrast group). Correspondingly the control SPI sample (referred to as CSPI, blank group) was prepared in a similar manner, just without the addition of protease. By employing the "subtraction" mode of logical thinking, we aimed to compare the differences between CSPI and D $\beta H$ in terms of dissociation/aggregation at $\mathrm{pH} 2.0$ with an ionic strength of $20 \mathrm{mmol} / \mathrm{L}$. Some state-of-theart and common techniques, such as transmission electron microscopy (TEM), dynamic light scattering (DLS), zeta potential measurement, intrinsic fluorescent spectrometry and circular dichroism (CD) spectrometry, were used to characterize the morphological and conformational changes of soy protein, as well as tetrahydrothiophene (Th T) dye was used to carry out binding studies. The knowledge gained from this study could better reveal the effect and mechanism of $\beta$-conglycinin on the fibril formation of SPI. 


\subsection{Materials and methods}

\subsubsection{Materials}

Cooled defatted soy flour was obtained from Qi Tian Biotechnology Co., Ltd. (Henan, China). The thioflavin T (Th T), bovine serum albumin (BSA), papain (21 units/mg) and antipain (Sigma nr A-6191) was purchased from Sigma-Aldrich Co. (St. Louis, Mo, USA). Bicinchoninic acid (BCA) protein assay kit was purchased from Shanghai Labaide biotechnology Co., Ltd. (Shanghai, China). Other reagents were obtained from SigmaAldrich Co. or Fisher Scientific Co. (Pittsburgh, PA, USA). All reagents used were of analytical grade or biochemical grade.

\subsubsection{Preparation of native soy protein isolate (SPI)}

To prepare SPI with their proteins in native state, procedures were followed according to the method reported by Wagner et al. [45]. Cooled defatted soy flour was extracted for $1.5 \mathrm{~h}$ at room temperature $\left(25^{\circ} \mathrm{C}\right.$ ) using deionized distilled water (DDW) (flour/water ratio was $1: 20$ ) and adjusted to $\mathrm{pH} 8.0$ with $2 \mathrm{~mol} / \mathrm{L} \mathrm{NaOH}$ solution. The suspension was centrifuged with $9000 \mathrm{~g}$ for $20 \mathrm{~min}$ at $4^{\circ} \mathrm{C}$ to retain the supernatant, which, in turn, was adjusted to $\mathrm{pH}$ 4.5 with $1 \mathrm{~mol} / \mathrm{L} \mathrm{HCl}$, kept for $2 \mathrm{~h}$ at $4{ }^{\circ} \mathrm{C}$, and then centrifuged again for 20 min at $4{ }^{\circ} \mathrm{C}$. The precipitate so obtained was redissolved in DDW to a protein concentration of $5 \%(\mathrm{w} / \mathrm{v})$ and neutralized to $\mathrm{pH} 7.5$ with $2 \mathrm{~mol} / \mathrm{L} \mathrm{NaOH}$ at $30^{\circ} \mathrm{C}$. Finally, the soy protein solution was put into molecular porous membrane tubing (MWCO: 12-14 kDa; Spectrum Medical Industries Inc., Houston, TX, USA) and dialyzed against reverse osmosis-purified water at 4 ${ }^{\circ} \mathrm{C}$ for $48 \mathrm{~h}$. The soy protein isolate so prepared was then freeze-dried.

\subsubsection{Preparation of soy protein subjected to selective proteolysis}

The soy protein hydrolysate subjected to selective proteolysis on $\beta$-conglycinin was obtained based on the method reported by Margatan et al. [39]. One liter of SPI dispersion (flour/water ratio of $1: 20$ ) was adjusted to $\mathrm{pH} 7.0$ with $2 \mathrm{~mol} / \mathrm{L} \mathrm{NaOH}$ solution and heated to $70^{\circ} \mathrm{C}$. Papain dissolved in DDW $(10 \mathrm{mg} / 2 \mathrm{~mL})$ was added, and the mixture was incubated at $70^{\circ} \mathrm{C}$ for $0-60$ min with gently stirring. The $\mathrm{pH}$ of the mixture was maintained throughout the incubation period by addition of $2 \mathrm{~mol} / \mathrm{L} \mathrm{NaOH}$. In the end, the reaction was terminated by adding a 10-fold molar amount of antipain, and the resulting hydrolysate was placed into molecular porous membrane tubing (MWCO: 12-14 kDa; Spectrum Medical Industries Inc., Houston, TX, USA) and dialyzed against $2 \mathrm{~L}$ of reverse osmosis purified water at $4{ }^{\circ} \mathrm{C}$. The water was changed every $2 \mathrm{~h}$ for the initial $6 \mathrm{~h}$ and then after $12 \mathrm{~h}$. Subsequently, the soy protein hydrolysate was lyophilized, referred to as "D $\mathbf{B H}$ ". In order to scientifically study the 
effects of selective proteolysis on $\beta$-conglycinin in SPI, the control SPI referred to as "CSPI" sample was prepared in a similar manner (incubated for $60 \mathrm{~min}$ ), just without the addition of protease [40].

\subsubsection{Degree of hydrolysis ( $\mathrm{DH})$}

The degree of hydrolysis was measured by the o-phthaldialdehyde (OPA) method as reported by Nielsen et al. [46], using DDW as the sample blank and L-serine as standard. The protein concentration of the samples was determined using the BCA method.

\subsubsection{Sodium dodecyl sulphate polyacrylamide gel electrophoresis (SDS-PAGE) analysis}

SDS-PAGE was performed on a discontinuous buffered system according to the method of Laemmli [47], using $12 \%$ separated gel and $5 \%$ stacking gel. The tested samples ( $3 \mathrm{mg} / \mathrm{mL}$ ) were prepared by mixing the lyophilized protein samples with SDS-PAGE sample buffer solution, namely, $0.0625 \mathrm{~mol} / \mathrm{L}$ Tris- $\mathrm{HCl}(\mathrm{pH} 8.0)$ containing $10 \%(\mathrm{v} / \mathrm{v})$ glycerol, $5 \%(\mathrm{v} / \mathrm{v})$ 2-mercaptoethanol, $2 \%(\mathrm{w} / \mathrm{v})$ SDS and $0.25 \%(\mathrm{w} / \mathrm{v})$ bromophenol blue. Aliquots $(15 \mu \mathrm{L})$ of the tested sample was loaded per well. The gels were stained with Coomassie Brilliant Blue $\mathrm{R}-250$ for $1 \mathrm{~h}$ followed by destaining. The changes in protein bands were estimated by analyzing electrophoretic gel images with Kodak 1D Image Analysis software 4.6 (Eastman Kodak Co., Rochester, NY, USA).

\subsubsection{Fibril aggregation experiment}

Heat-induced aggregation experiments followed the method reported by Tang et al. [48]. Protein solutions with a protein concentration of $1.0 \mathrm{mg} / \mathrm{mL}$ at $\mathrm{pH} 2.0$ were prepared by dissolving the lyophilized sample into $\mathrm{pH} 2.0 \mathrm{HCl}$ solution with an ionic strength of $20 \mathrm{mmol} / \mathrm{L}$ and then accurately adjusting the solutions to $\mathrm{pH} 2.0$ with $6 \mathrm{~mol} / \mathrm{L} \mathrm{HCl}$ solution. The protein concentration of the solution was determined using the BCA method. Aliquots $(5 \mathrm{~mL})$ of protein solutions were put into tubes with hermetic lids. The tubes were heated at $95{ }^{\circ} \mathrm{C}$ for specific periods of time ( $0 \mathrm{~min}, 15 \mathrm{~min}, 60 \mathrm{~min}, 360 \mathrm{~min}$ and $720 \mathrm{~min}$ ) in a temperaturecontrolled bath with a temperature deviation of $<1^{\circ} \mathrm{C}$. After the heat treatment, the samples were immediately cooled to $25 \pm 1{ }^{\circ} \mathrm{C}$ in an ice-water bath and directly subjected to further experiments. 


\subsubsection{Particle size measurement}

The freshly prepared protein samples were diluted to $0.1 \mathrm{mg} / \mathrm{mL}$ with $\mathrm{pH} 2.0 \mathrm{HCl}$ solution. The test solutions were placed in a $1 \mathrm{~cm} \times 1 \mathrm{~cm}$ disposable cuvette (model: DTS0012). DLS analysis was performed with a backscattering angle of $173^{\circ}$ and a refractive index of 1.41 for protein particles using a Zetasizer Nano ZS (Malvern Instrument Ltd., Worcestershire, U.K.) at $25^{\circ} \mathrm{C}$. The particle size was described by the volume-weighted mean diameter and determinations were conducted in triplicate.

\subsubsection{Determination of zeta potential}

The freshly prepared protein samples were diluted to $0.1 \mathrm{mg} / \mathrm{mL}$ with $\mathrm{pH} 2.0 \mathrm{HCl}$ solution with an ionic strength of $20 \mathrm{mmol} / \mathrm{L}$. Zeta potential of the test sample was determined in triplicate (input voltage is $-5 \mathrm{~V} \sim+5 \mathrm{~V}$, the dielectric constant is $78.5 \mathrm{~F} / \mathrm{m}$ for water, the refractive index is 1.33 for water) using Zetasizer Nano ZS (Malvern Instrument Ltd., Worcestershire, U.K.)

\subsubsection{Circular dichroism (CD) spectroscopy}

The freshly prepared protein samples were diluted to $0.05 \mathrm{mg} / \mathrm{mL}$ with $\mathrm{pH} 2.0 \mathrm{HCl}$ solution with an ionic strength of $20 \mathrm{mmol} / \mathrm{L}$. Far-UV CD spectral data were obtained using a J-1500 CD spectropolarimeter (Jasco Corp., Tokyo, Japan). The spectroscopic measurements were performed in a $0.1 \mathrm{~cm}$ quartz cuvette with a protein concentration of around $0.05 \mathrm{mg} / \mathrm{mL}$ in $\mathrm{HCl}$ solution $(\mathrm{pH} 2.0)$. The protein concentrations were determined using the BCA method. The protein samples were scanned from 190 to $250 \mathrm{~nm}$. The scan rate, response and bandwidth were $50 \mathrm{~nm} / \mathrm{min}, 4 \mathrm{~s}$ and $1.0 \mathrm{~nm}$, respectively. Recorded spectra were an average of three scans and corrected by subtraction of the spectrum of a protein-free buffer. A mean value of 110 for the amino acid residue was assumed in all calculations, and CD measurements were expressed as mean residue ellipticity $(\theta)$ in $\operatorname{deg} \mathrm{cm}^{-2} \mathrm{dmol}^{-1}$. The proportion of four secondary structures ( $a$-helix, $\beta$-sheet, $\beta$-turn, random coil) was derived from Yang's equation.

\subsubsection{Th T fluorescence spectral analysis}

Th $\mathrm{T}$ stock solution was prepared by dispersing $8 \mathrm{mg}$ Th $\mathrm{T}$ into $10 \mathrm{~mL}$ of $10 \mathrm{mmol} / \mathrm{L}$ phosphate buffer $(\mathrm{pH} \mathrm{7.0)}$ containing $150 \mathrm{mmol} / \mathrm{L} \mathrm{NaCl}$. The dispersion was filtered with a $0.2 \mu \mathrm{m}$ syringe filter to remove undissolved $\mathrm{Th} \mathrm{T}$. This stock solution should be stored in the dark at $4^{\circ} \mathrm{C}$ and covered with aluminum foil. The stock solution was diluted 50 times in the same buffer on the day of analysis to generate the working solution. Aliquots $(50 \mu \mathrm{L})$ of the 
protein solutions were mixed with $5 \mathrm{~mL}$ of the Th T working solution and allowed to stand for $1 \mathrm{~min}$. The fluorescence spectra of the mixtures were measured using a Hitachi F-4600 fluorescence spectrophotometer (Hitachi, Ltd., Tokyo, Japan). The excitation wavelength was set at $460 \mathrm{~nm}$ (slit width $=10 \mathrm{~nm}$ ) and the emission spectra were obtained from 450 to $600 \mathrm{~nm}$ with a scanning speed of $240 \mathrm{~nm} / \mathrm{min}$. The fluorescence spectrum of the Th T working solution was subtracted from that of the samples to correct the background signal [48].

\subsubsection{Transmission electron microscopy (TEM)}

The test solutions $(1.0 \mathrm{mg} / \mathrm{mL})$ applied for the TEM observation were diluted 10 times with $\mathrm{HCl}$ solution ( $\mathrm{pH} 2.0$ with an ionic strength of $20 \mathrm{mmol} / \mathrm{L}$ ), and a droplet of diluted solution was put onto a carbon support film on a copper grid. After $30 \mathrm{~min}$, the droplet was removed using filter paper. Then a drop of $1 \%(\mathrm{w} / \mathrm{v})$ uranyl acetate was put onto the grid and removed after $30 \mathrm{~s}$. The micrographs were prepared at $80 \mathrm{kV}$ using a Hitachi $\mathrm{H}-7650$ transmission electron microscope (Hitachi, Ltd., Tokyo, Japan).

\subsubsection{Intrinsic fluorescence emission spectroscopy}

Intrinsic fluorescence emission spectra of the protein samples were obtained by a Hitachi F4600 fluorescence spectrophotometer (Hitachi, Ltd., Tokyo, Japan). Protein solutions were diluted in $\mathrm{HCl}$ solution ( $\mathrm{pH} 2.0$ with an ionic strength of $20 \mathrm{mmol} / \mathrm{L}$ ) to a protein concentration of $0.1 \mathrm{mg} / \mathrm{mL}$. The excitation wavelength was set at $290 \mathrm{~nm}$ (slit width $5 \mathrm{~nm}$ ) and the emission spectra were obtained from 300 to $400 \mathrm{~nm}$ with a scanning speed of 240 $\mathrm{nm} / \mathrm{min}$.

\subsubsection{Statistical analysis values}

Values given in the tables and figures are the means of triplicates, and error bars indicated the standard deviation. The statistical significance of differences among means was evaluated by Duncan's test at $p<0.05$. Statistical analysis was performed using SPSS software version 22.0 (Statistical Package for Social Sciences, IBM, New York, USA).

\subsection{Results and discussion}

\subsubsection{Selective proteolysis of $\beta$-conglycinin in SPI}

The SDS-PAGE patterns (Fig. 2.1) showed that the bands corresponding to $\beta$-conglycinin gradually faded over time whereas the bands corresponding to glycinin were not affected, according to the gel electrophoresis densitometric scan, confirming the selective proteolysis 
on $\beta$-conglycinin was successfully achieved in SPI. This was because $\beta$-conglycinin in native SPI could be gradually and properly hydrolyzed at a reaction temperature at $60-70{ }^{\circ} \mathrm{C}$, which was around the optimum temperature of papain; glycinin in SPI was adequately resistant to hydrolysis when the reaction temperature was below $80^{\circ} \mathrm{C}[31,38]$.

The relative content of $\beta$-conglycinin and its subunits $\left(a, a^{\prime}, \beta\right)$ in lane 2-7 were calculated with CSPI (lane 1) as standard. Compared with CSPI, the aa' subunit contents of selective hydrolysates (lanes 2-7) were reduced to 59\%, 46\%, 44\%, 42\%, 36\% and 31\%, respectively of their initial value; the $\beta$ subunit contents of the selective hydrolysates were reduced to $49 \%, 48 \%, 38 \%, 31 \%, 26 \%$, and $13 \%$, respectively. Totally, the $\beta$-conglycinin contents of selective hydrolysates were reduced to $58 \%, 46 \%, 43 \%, 41 \%, 34 \%$, and $28 \%$, respectively (Fig. 2.1). In the present experiment, the soy protein sample in lane 7 whose DH was $4 \%$ was adopted as the subsequent selective hydrolysate sample.

Before our present experiment, in 2005, Tsumura et al. reported that the densitometric analysis after SDS-PAGE showed that the reduction of $\beta$-conglycinin content in SPI was $92 \%$ after selective hydrolysis $\left([\mathrm{E}] /[\mathrm{S}]=4.20 \mathrm{units} / \mathrm{g}\right.$, the reaction temperature was $70{ }^{\circ} \mathrm{C}$, reaction time was $30 \mathrm{~min}$ ) [40]. In 2013, Margatan et al. reported that $\beta$-conglycinin in SPI was almost selectively hydrolyzed thoroughly with only a few subunits of $\beta$-conglycinin remaining $\left([E] /[S]=3.99\right.$ units $/ g$, the reaction temperature was $70^{\circ} \mathrm{C}$, reaction time was $30 \mathrm{~min}$ ) [39]. In our experiment, the densitometric analysis after SDS-PAGE showed that the reduction of $\beta$-conglycinin in SPI was $72 \%([E] /[S]=4.20$ units $/ g$, the reaction temperature was $70^{\circ} \mathrm{C}$, reaction time was $60 \mathrm{~min}$ ). The differences in the degradation rate of $\beta$-conglycinin in SPI between our and other experiments on selective proteolysis should attribute to the variety of soy protein isolate sources. Many studies have revealed that the subunits composition and conformational structures of soy protein would make a difference related to soybean cultivars, soil environment, climatic conditions, post-harvest storage conditions (temperature, humidity, storage time), and so on[49-54]. Finally, the reason why we did not prolong the reaction time to exceed $60 \mathrm{~min}$ was that long-time incubation at elevated temperature could lead to a substantial loss of secondary and tertiary structures, which would deteriorate the effectiveness of selective proteolysis [39]. 


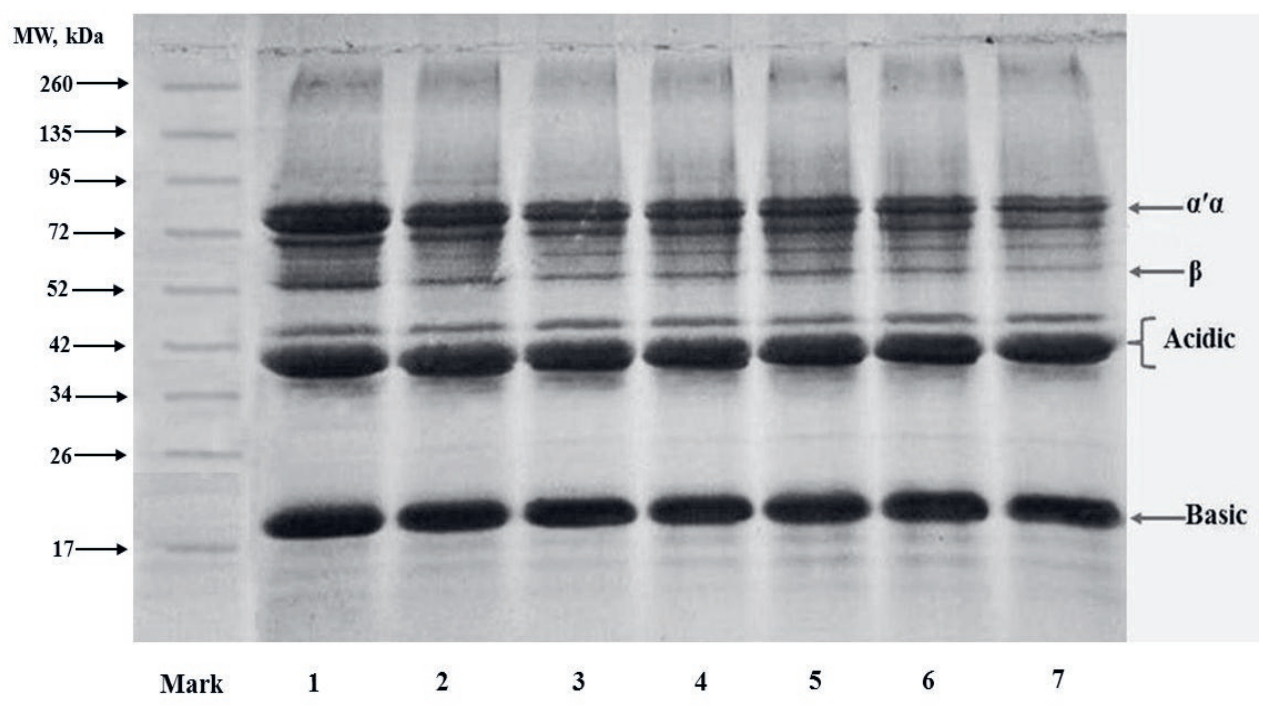

Figure 2.1. SDS-PAGE patterns of the hydrolysates prepared with papain at $\mathrm{pH} 7.0$ and $70{ }^{\circ} \mathrm{C}$, at various reaction times. lane 1: CSPI; lane 2: $10 \mathrm{~min}$; lane 3: $20 \mathrm{~min}$; lane 4: $30 \mathrm{~min}$; lane 5: $40 \mathrm{~min}$; lane 6: $50 \mathrm{~min}$; lane 7: $60 \mathrm{~min}$.

\subsubsection{Particle size measurement}

As a sensitive and quantitative technique, DLS is widely applied to monitor the formation of protein aggregates [43,55]. Fig. 2.2 showed that when the incubation time was 0, 15, 60, 360 and $720 \mathrm{~min}$, the average particle size of CSPI and DBH brought out a similar variation. Prior to heating, the average particle size of $\mathrm{D} \beta \mathrm{H}(194 \pm 8 \mathrm{~nm})$ was smaller than that of CSPI $(238 \pm 9 \mathrm{~nm})$, indicating that the proteolysis reduced the average particle size of soy protein. When heated for $15 \mathrm{~min}$, the average particle size of both CSPI and DBH decreased to $\sim 116 \mathrm{~nm}$. Most likely, the initial short time heating reduced protein molecules to smaller fragments or oligopeptides, which could be called "fibrillation preparatory stage". Nijboer et al. reported that the $\beta$-lactoglobulin fibrils formed at $\mathrm{pH} 2.0$ were composed of peptides and that the formation of peptides preceded their incorporation into the fibrils [56]. Akkermans et al. claimed that heat-induced fibrillation through self-assembly under acidic conditions was related to the change of protein conformation and hydrolysis of peptides, which were the underlying requirements for the formation of protein fibrils [57]. Similar disruption of proteins by heating at acid condition also had been observed in whey protein isolate [58], phaseolin [48], and lysozyme [59]. 


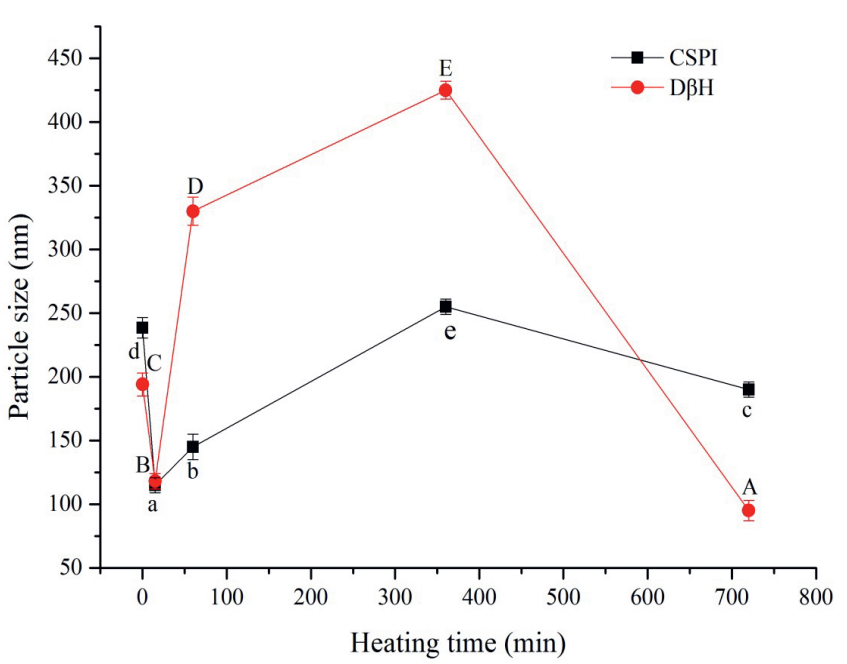

Figure 2.2. Particle size profiles of CSPI and $\mathrm{D} \beta \mathrm{H}$, heated $\left(95^{\circ} \mathrm{C}\right.$ ) at pH 2.0 and ionic strength of $20 \mathrm{mmol} / \mathrm{L}$ for $0,15,60,360,720 \mathrm{~min}$, respectively. Each data is the means and standard deviations of five measurements. Different letters indicate significant differences $(p<0.05)$ : CSPI, a e; D BH, A E.

When heated for $60 \mathrm{~min}$, the average particle size of CSPI and D $\beta H$ increased significantly (145 \pm 11 and $330 \pm 10 \mathrm{~nm}$, respectively, $p<0.05)$, indicating that the proteins fragments and (or) short peptides associated together to form aggregates again, and this stage could be called "fibrillation emergence stage". When heated for $360 \mathrm{~min}$, the average particle sizes of CSPI and DBH further increased to $255 \pm 7$ and $425 \pm 6 \mathrm{~nm}$, respectively, which would be the "fibrillation development stage". As the heating time reached up to $720 \mathrm{~min}$, the average particle sizes of CSPI and D $B H$ dramatically decreased again to $190 \pm 8$ and $95 \pm$ $6 \mathrm{~nm}$, respectively, which could be assigned to the "fibrillation reverse stage".

\subsubsection{Circular dichroism spectrum (CD) analysis}

Protein secondary structures can be divided into four types, namely, a-helix, $\beta$-sheet, $\beta$-turn and random coil. $a-H e l i x$ and $\beta$-sheet are ordered secondary structures characterized by high rigidity and a repetitive structure; while $\beta$-turn and random coil are unordered secondary structures characterized by flexibility and the absence of repetitive structure.

Table 2.1 showed that prior to heating, compared with CSPI, D $\beta H$ contained more a-helix structure $(23.6 \%$ vs. $13.2 \%)$ and random coil structure $(52.3 \%$ vs. $48.4 \%)$. And more remarkably, the $\beta$-turn structure disappeared in $D \beta H$. These could be ascribed to the following reasons: (1) selective proteolysis of SPI led to the rearrangement of chemical bonds and the reorganization of conformational structures; (2) the $\beta$-turn structure mainly appeared in the $\beta$-globulin molecules and tended to exist in or close to the protein molecule 
surface, which meant the proteolysis would very likely destroy the $\beta$-turn structure of $\beta$ globulin $[60,61]$.

When heated for $15 \mathrm{~min}$, a significant increase of a-helix structure and $\beta$-turn structure, and a significant reduction of $\beta$-sheet structure and random coil structure were observed in both CSPI and DBH $(p<0.05)$. When heated for $60 \mathrm{~min}$, the total amount of ordered secondary structure (i.e., $a$-helix and $\beta$-sheet) of both CSPI and D $\beta H$ increased significantly. Among them, the $\beta$-sheet structure of CSPI increased from $21.9 \%$ to $64.3 \%$ and that of $\mathrm{D} \beta \mathrm{H}$ increased from $18.4 \%$ to $51.0 \%$. Meanwhile, the $\beta$-turn structure disappeared in both CSPI and $\mathrm{D} \beta \mathrm{H}$. Tang et al. found that the formation of linear aggregates or amyloid-like fibrils involved a process of disruption and reorganization of the ordered secondary structures [48]. These results might suggest the initiation of protein fibrillations since the $\beta$-sheet structure was one of the criteria to define the formation of amyloid fibrils [5]. Some other researchers also found that the $\beta$-sheet structure content of bovine serum albumin and myosin remarkably increased when the proteins assembled into amyloid fibrils under heating $[62,63]$. When heated for $360 \mathrm{~min}$, the $\beta$-sheet structure of CSPI began to decrease, while the $\beta$-sheet of $D \beta H$ continued to increase. Whereafter, the $\beta$-sheet structure almost completely disappeared in both CSPI and D $\beta H$ after heating for $720 \mathrm{~min}$. And the unordered structure (i.e., $\beta$-turn and random coil) dominated the second structure.

Table 2.1. The secondary structure compositions of protein fibrils in $\mathrm{DBH}$ and CSPI solutions. Heating at $95^{\circ} \mathrm{C}$ for $0,15,60,360,720 \mathrm{~min}$, respectively. Different letters (a-e) in the same column indicate significant differences $(p<0.05)$. Variation of the mean represents standard deviation of triplication for each sample.

(A)

\begin{tabular}{lllll}
\hline $\mathrm{D} \beta \mathrm{H}$ & $\mathrm{a}$-helix & $\beta$-sheet & $\beta$-turn & random coil \\
\hline $0 \mathrm{~min}$ & $23.63 \pm 0.15 \mathrm{~b}$ & $24.10 \pm 0.02 \mathrm{c}$ & $0.00 \pm 0.00 \mathrm{c}$ & $52.32 \pm 0.21 \mathrm{a}$ \\
$15 \mathrm{~min}$ & $27.22 \pm 0.21 \mathrm{a}$ & $18.42 \pm 0.11 \mathrm{~d}$ & $20.72 \pm 0.25 \mathrm{~b}$ & $33.73 \pm 0.15 \mathrm{e}$ \\
$60 \mathrm{~min}$ & $12.10 \pm 0.12 \mathrm{c}$ & $51.02 \pm 0.34 \mathrm{~b}$ & $0.00 \pm 0.00 \mathrm{c}$ & $37.00 \pm 0.09 \mathrm{~d}$ \\
$360 \mathrm{~min}$ & $0.60 \pm 0.03 \mathrm{e}$ & $55.51 \pm 0.15 \mathrm{a}$ & $0.00 \pm 0.00 \mathrm{c}$ & $43.91 \pm 0.12 \mathrm{~b}$ \\
$720 \mathrm{~min}$ & $6.60 \pm 0.09 \mathrm{~d}$ & $0.00 \pm 0.00 \mathrm{e}$ & $52.91 \pm 0.16 \mathrm{a}$ & $40.52 \pm 0.15 \mathrm{c}$ \\
\hline
\end{tabular}


Table 2.1. (B)

\begin{tabular}{lllll}
\hline CSPI & a-helix & $\beta$-sheet & $\beta$-turn & random coil \\
\hline 0 min & $13.21 \pm 0.22 \mathrm{~b}$ & $25.00 \pm 0.13 \mathrm{c}$ & $13.53 \pm 0.09 \mathrm{c}$ & $48.41 \pm 0.13 \mathrm{c}$ \\
$15 \mathrm{~min}$ & $16.32 \pm 0.14 \mathrm{a}$ & $21.91 \pm 0.08 \mathrm{~d}$ & $37.52 \pm 0.13 \mathrm{a}$ & $24.32 \pm 0.07 \mathrm{e}$ \\
$60 \mathrm{~min}$ & $4.71 \pm 0.05 \mathrm{e}$ & $64.32 \pm 0.21 \mathrm{a}$ & $0.00 \pm 0.00 \mathrm{~d}$ & $31.12 \pm 0.05 \mathrm{~d}$ \\
$360 \mathrm{~min}$ & $4.36 \pm 0.11 \mathrm{~d}$ & $60.51 \pm 0.16 \mathrm{~b}$ & $0.00 \pm 0.00 \mathrm{~d}$ & $35.13 \pm 0.12 \mathrm{~b}$ \\
$720 \mathrm{~min}$ & $12.41 \pm 0.37 \mathrm{c}$ & $4.00 \pm 0.03 \mathrm{e}$ & $34.82 \pm 0.15 \mathrm{~b}$ & $48.91 \pm 0.14 \mathrm{a}$ \\
\hline
\end{tabular}

\subsubsection{Zeta potential analysis}

The zeta potential refers to the potential of the shear layer on the surface of charged particles and is widely used to describe the electrostatic interaction between colloidal particles. The zeta potential is dependent on both the surface charges and the microenvironment of particles. Thus, compared with surface potential, the zeta potential is more suitable in describing the interaction between charged particles in solution [64]. The net charge is a requisite for protein to form amyloid fibrils through self-assembly [65]. At $\mathrm{pH} 2.0$, the dissociation of carboxyl was suppressed while the corresponding amidogen was protonated, therefore the soy protein solution systems were positively charged.

Fig. 2.3 showed that prior to heating, the zeta potential of $\mathrm{D} \beta H(22.13 \pm 0.9 \mathrm{mV})$ was higher than that of CSPI $(20.70 \pm 0.6 \mathrm{mV})$, which might be because proteolysis led to a reduction of the hydrophobic microdomain of the protein and an increase in the interactions between polar portions of proteins and water [66]. During the whole incubation period, the zeta potential of CSPI and that of $\mathrm{D} \beta \mathrm{H}$ displayed a similar variation tendency: they decreased dramatically at the initial stage of heating and reached a minimum value at $360 \mathrm{~min}$, and then rose as heating time increased (Fig. 2.3). During the heating process oxidation reactions could occur. The oxidation reactions could have caused protein structures partly to unfold which reduced the surface charge density of proteins and electrostatic repulsive interaction between polypeptide chains $[67,68]$. Consequently, the partly unfolded polypeptides came close to each other and tended to assemble into protein fibrils [69]. However, protein particles possessing too low electrostatic repulsion did not benefit fibrillation, but instead would induce protein particles to form disordered clusters $[55,70,71]$. Overall, the electrostatic repulsion between the peptides or building blocks was an important 
determining parameter for the fibril assembly. Only the proper balance between hydrophobic and electrostatic repulsion interaction could promote the formation of protein fibrils $[43,72]$.

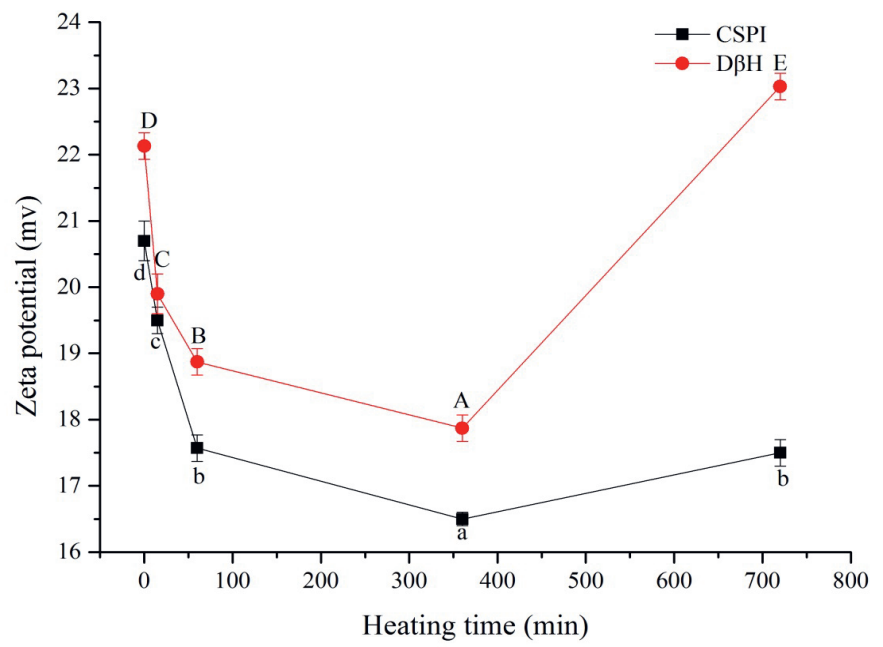

Figure 2.3. Zeta potential profiles of CSPI and $D \beta H$, heated $\left(95^{\circ} \mathrm{C}\right)$ at $\mathrm{pH} 2.0$ and ionic strength of $20 \mathrm{mmol} / \mathrm{L}$ for $0,15,60,360,720 \mathrm{~min}$, respectively. Different letters indicate significant differences $(p<0.05)$ : CSPI, a d; $D \beta H, A \sim E$.

\subsubsection{Thioflavin T spectroscopic assays}

Th $\mathrm{T}$ is a kind of cationic benzothiazole, and its fluorescence intensity will rise when being combined with the $\beta$-sheet structure regions of amyloid fibrils $[5,6,57]$. Saeed and Fine (1967) compared the effectiveness of different dyes, such as Congo red, crystal violet, Van Gieson, and Th T, in identifying protein amyloid fibrils and finally the Th T turned out to be the best among them [73].

The nature of the aggregates formed from CSPI and DBH at specific time intervals (0-720 min) was characterized using Th $T$ spectroscopic assays. As shown in Fig. 2.4, the fluorescence intensity of both CSPI and D $\beta H$ were very low at the initial incubation time (0$15 \mathrm{~min}$ ). When heated for $60 \mathrm{~min}$, the maximum fluorescence intensity of both CSPI and $D \beta H$ remarkably increased, which could indicate changes of the protein secondary and tertiary structure (e.g., the $\beta$-sheet structure became dominant) and the formation of amyloid fibrils [73-76]. Thereafter, the Th T maximum fluorescence intensity of both CSPI and $D \beta H$ decreased with heating time from 60 to $360 \mathrm{~min}$. Given the still relatively high $\beta$ sheet structure proportion and a significant increase in particle size of both CSPI and D $\beta H$ during this period, the decrease of Th T maximum fluorescence intensity could be attributed to the entanglement of formed fibrils into relatively unordered aggregates $[48,77,78]$. In the end, the ThT maximum fluorescence intensity of both CSPI and D $\beta H$ decreased to a very 
low level again when heated for $720 \mathrm{~min}$, which could be due to the formed amyloid fibrils being transformed to other types of aggregates and/or monomers.

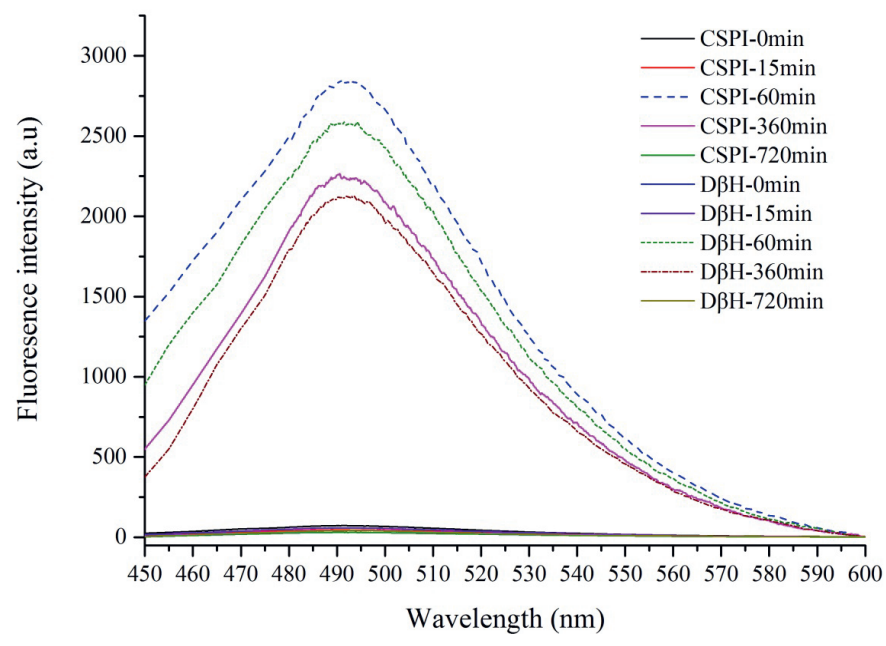

Figure 2.4. Th T spectroscopic profiles of CSPI and D BH, heated $\left(95^{\circ} \mathrm{C}\right)$ at $\mathrm{pH} 2.0$ and ionic strength of 20 $\mathrm{mmol} / \mathrm{L}$ for $0,15,60,360,720 \mathrm{~min}$, respectively

\subsubsection{Transmission electron microscope (TEM) analysis}

Fig. 2.5 shows the TEM micrographs of CSPI and D $3 H$ heated for $0,15,60,360$ and 720 min at pH 2.0 with the ionic strength of $20 \mathrm{mmol} / \mathrm{L}$. Prior to heating, the CSPI particles ( $=240 \mathrm{~nm}$ in average contour diameter) were regular spherical, whereas the $\mathrm{D} \beta H$ particles (=190 $\mathrm{nm}$ in average contour diameter) were spherical-like with serrated margins, which could be caused by proteolysis (Fig. 2.5A, F). When heated for 15 min (Fig. 2.5B, G), CSPI and $\mathrm{D} \beta \mathrm{H}$ particles obviously degraded, producing many smaller particles with various sizes and an uneven distribution, which was consistent with the reduction in average diameter measured by DLS.

When heated for $60 \mathrm{~min}$ (Fig. 2.5C, H), short worm-like fibrils with an average length of 135 $\mathrm{nm}$ and width of $14 \mathrm{~nm}$ were observed in CSPI (a small amount of agglomerates with an average particle size of $67 \mathrm{~nm}$ were also observed). The coexistence of various forms of protein aggregates was not surprising. Min et al. proposed that protein aggregation could be very complex, involving a variety of different oligomeric intermediates whose population would be determined by the kinetic and thermodynamic competition between them [77]. Long semiflexible fibrils with an average length of $335 \mathrm{~nm}$ and width of $10 \mathrm{~nm}$ were observed from $D \beta H$. Tang and Wang compared the formation of heat-induced fibrils from $\beta$-conglycinin and glycinin, and found that individual $\beta$-conglycinin was prone to forming worm-like fibrils 
while most fibrils from individual glycinin were in a long semiflexible configuration [7]. These above findings indicated that different soy proteins usually tended to form protein fibrils with different morphologies under acidic conditions, which was related to the diversities in structure development, subunit composition and polypeptide chain hydrolysis [79-81].

When heated for $360 \mathrm{~min}$ (Fig. 2.5D, I), fibril clusters were clearly observed from CSPI (= an average length and width of $270 \mathrm{~nm}$ and $35 \mathrm{~nm}$, respectively) and D $\beta H$ (= an average length and width of $400 \mathrm{~nm}$ and $67 \mathrm{~nm}$, respectively). The fibril clusters from CSPI were relatively short and present in a twisted form, whereas the fibril clusters from $D B H$ were relatively long and associated into bundles. The appearance of co-aggregates could be due to the low surface charge density benefiting the attraction between polypeptides and accelerating the cluster reaction $[55,82]$.

When heated for $720 \mathrm{~min}$, the formed fibrils clusters had disappeared, and instead of these, non-fibrillar aggregates were observed in both samples. In CSPI, the morphologies of these aggregates were mainly elliptical and amorphous, and the contour diameters of them varied from $120 \mathrm{~nm}$ to $535 \mathrm{~nm}$. In $\mathrm{D} \beta \mathrm{H}$, the morphologies of these aggregates were relatively small, and were mainly clubbed (average contour length and width was $50 \mathrm{~nm}$ and $5 \mathrm{~nm}$ ) and amorphous (average contour diameter was $67 \mathrm{~nm}$ ). The reverse reaction of fibril formation occurred during this period, and the formed fibrils disintegrated into monomers and oligomers again. Since these released protein particles were unstable, some of them reassembled to non-linear aggregates with diverse sizes and morphologies. Veerman and colleagues proposed that at a high protein concentration, proteins were more likely to link with each other via chemical bonding which might suppress the reverse reaction [83], and the reverse reaction of the protein fibrillation usually occurred at a low protein concentration. 


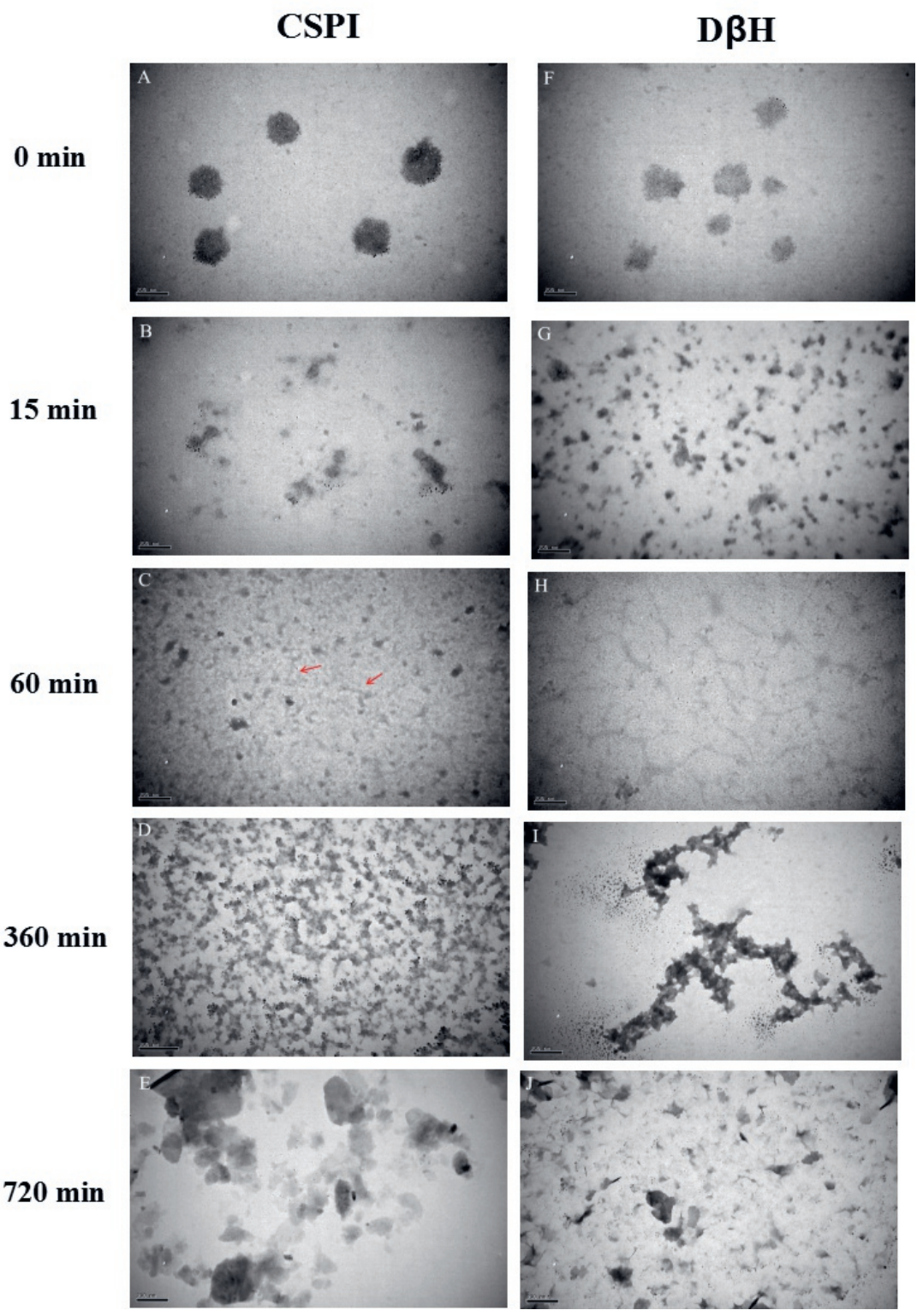

Figure 2.5 TEM micrographs of amyloid fibrils in CSPI and DBH solutions heated at $95{ }^{\circ} \mathrm{C}$ for various time, $A$ :

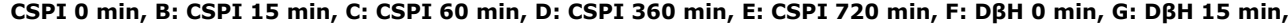

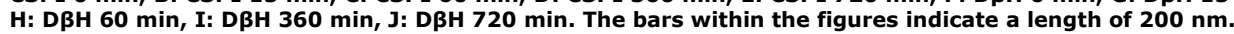




\subsubsection{Intrinsic fluorescence emission spectra}

Fluorescence spectra are a useful tool to monitor tertiary structure changes in proteins because the intrinsic fluorescence of aromatic amino acid residues is sensitive to the polarity of their microenvironment [84]. The fluorescence spectrum was mainly emitted from tryptophan (Trp) residues when the excitation wavelength was $290 \mathrm{~nm}$ and the observed maximum emission wavelength $\left(\boldsymbol{\lambda}_{\max }\right)$ for Trp residues in protein ranges from 308 to 355 $\mathrm{nm}$ [85]. The changes of $\lambda_{\max }$ and fluorescence intensity (FI) from CSPI or D $\beta H$ as a function of incubation time are shown in Fig. 2.6 and Table 2.2. Prior to heating, D $\beta H$ showed stronger FI than CSPI, which could be due to proteolysis altering the microenvironment of tryptophan in SPI [86]. When heated for $15 \mathrm{~min}, \lambda_{\max }$ of both CSPI and D $\beta H$ displayed a red shift to longer wavelengths. Referring to our foregoing DLS analysis, the red shift of $\lambda_{\max }$ could be caused by the degradation of protein in the preparatory stage of fibrillation, exposing more tryptophan. Lakemond et al. reported that when soy glycinin degraded into its subunits, the rigid structure of glycinin was disrupted and the $\lambda_{\max }$ of tryptophan displayed a concomitant red shift [86]. In addition, the FI of CSPI was found increased, whereas that of D $\beta H$ was found decreased $(p<0.05)$. The different changes of FI between CSPI and D $\beta H$ could be on account of the difference in the microenvironment of Trp in SPI during the disruption of peptide chains at $\mathrm{pH}$ 2.0. When incubated for $60 \mathrm{~min}, \lambda_{\max }$ of CSPI and D $3 H$ both displayed a blue shift with an accompanied decrease of fluorescence intensity $(p<0.05)$. These revealed that the microenvironments of Trp residues became more hydrophobic, as a result of protein fibrillation. Thereafter, the $\lambda_{\max }$ of CSPI and DBH both displayed a red shift to longer wavelengths again when the heating time was at $360 \mathrm{~min}$. At last, when heated for $720 \mathrm{~min}$, the $\lambda_{\max }$ of both CSPI and D $3 \mathrm{H}$ displayed a blue shift accompanied by a declining fluorescence intensity $(p<0.05)$. In general, during the latter period of incubation ( $>60$ min), fluorescence intensity declined continuously. Under the heat treatment, the FI of Trp residues depended on not only the embedding extent of them but also the heat-induced oxidation of Trp. Since the Trp residue was vulnerable to oxidation reactions, the heatinduced oxidation could quench their FI dramatically [87-89]. 

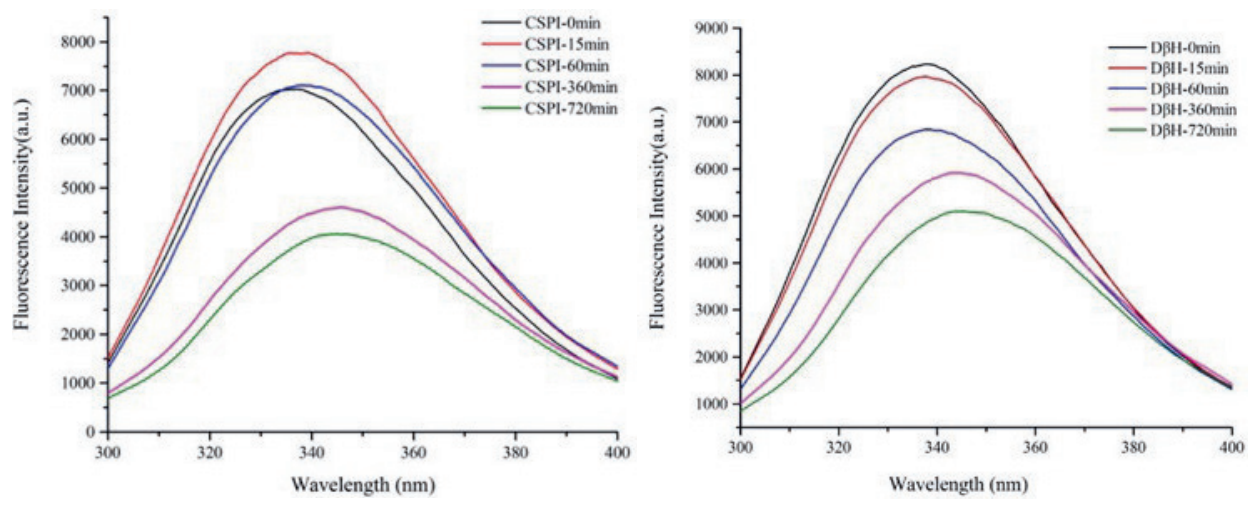

Figure 2.6 Intrinsic fluorescence emission spectra of CSPI (A) and D $\beta H(B)$, heated $\left(95^{\circ} \mathrm{C}\right)$ at $\mathrm{pH} 2.0$ and ionic strength of $20 \mathrm{mmol} / \mathrm{L}$ for $0,15,60,360,720 \mathrm{~min}$, respectively.

Table 2.2 The $\lambda_{\max }$ and maximum fluorescence intensity of emission fluorescence spectroscopic of $D \beta H$ and CSPI solutions. Heating at $95^{\circ} \mathrm{C}$ for $0,15,60,360,720 \mathrm{~min}$, respectively. Different letters (a-e) in the same column indicate significant differences $(p<0.05)$. Variation of the mean represents standard deviation of triplication for each sample.

\begin{tabular}{|c|c|c|c|c|}
\hline & \multicolumn{2}{|c|}{ CSPI } & \multicolumn{2}{|c|}{$D \beta H$} \\
\hline & $\lambda_{\max }(\mathrm{nm})$ & FI (a.u.) & $\lambda \max (\mathrm{nm})$ & FI (a.u.) \\
\hline $0 \mathrm{~min}$ & $333.4 \pm 0.5 a$ & $7337 \pm 41 c$ & $334.4 \pm 0.8 a$ & $8454 \pm 59 e$ \\
\hline $15 \mathrm{~min}$ & $339.8 \pm 0.9 c$ & $8041 \pm 35 d$ & $339.2 \pm 0.5 c$ & $8200 \pm 40 d$ \\
\hline $60 \mathrm{~min}$ & $335.4 \pm 1.2 b$ & $7268 \pm 67 c$ & $337.7 \pm 0.8 b$ & $7047 \pm 49 c$ \\
\hline $360 \mathrm{~min}$ & $349.6 \pm 1.4 d$ & $4726 \pm 54 b$ & $347.0 \pm 1.1 \mathrm{e}$ & $6102 \pm 50 \mathrm{~b}$ \\
\hline $720 \mathrm{~min}$ & $340.2 \pm 0.9 c$ & $4186 \pm 60 a$ & $345.4 \pm 0.7 d$ & $5277 \pm 37 a$ \\
\hline
\end{tabular}




\subsection{Conclusions}

The amyloid fibril formation of CSPI and $\mathrm{D} \beta \mathrm{H}$ were multistage under heating at $\mathrm{pH} 2.0$ with an ionic strength of $20 \mathrm{mmol} / \mathrm{L}$. At $15 \mathrm{~min}$, both CSPI and DBH first degraded into building blocks of protein fibrils with the particle size and zeta potential dropping significantly. At 60 min, short worm-like fibrils were observed by CSPI and long semiflexible fibrils were observed in $\mathrm{D} \beta \mathrm{H}$. Concomitantly, the $\beta$-sheet structure and Th T fluorescence intensity dramatically increased. At $360 \mathrm{~min}$, the fibrils formed from both formed clusters. Prolonging heating time to $720 \mathrm{~min}$, the formed protein fibrils dissociated into monomers and/or oligomers again. In brief, the absence of $\beta$-conglycinin in $D \beta H$ could be responsible for the different morphologies of aggregates and different conformation changes of protein compared to CSPI. Further research would be desirable to explore the effects of the varying amounts of $\beta$-conglycinin in SPI on the formation of amyloid fibrils. 


\section{Chapter 3}

High-intensity ultrasound treatment on soy protein after selectively proteolyzing glycinin component: Physical, structural, and aggregation properties

\section{Published as:}

W. Xia, S. Pan, Z. Cheng, Y. Tian, X. Huang, High-intensity ultrasound treatment on soy protein after selectively proteolyzing glycinin component: Physical, structural, and aggregation properties, Foods. 9 (2020) 839. doi:10.3390/foods9060839 


\section{Abstract}

In this study, a novel method called selective proteolysis was applied to the glycinin component of soy protein isolate (SPI), and a degraded glycinin hydrolysate (DGH) was obtained. The effects of high-intensity ultrasound (HIU) treatment $(20 \mathrm{kHz}$ at $400 \mathrm{~W}, 0,5$, 20 , and $40 \mathrm{~min}$ ) on the physical, structural, and aggregation properties of DGH were investigated with the aim to reveal the influence of the selectively hydrolyzing glycinin on the HIU treatment of soy protein. The effects of HIU on DGH and a control SPI (CSPI) were both time-dependent. HIU induced the formation of soluble aggregates in both samples at an early stage, while it dissociated these newly formed aggregates after a longer duration. Selectively hydrolyzing glycinin contributed to soluble aggregate formation by exposing the compact protein structure and producing small protein fractions. The larger extent of hydrophobic interactions and disulfide bonds imparted higher stability to the soluble protein aggregates formed in DGH. As a result, DGH displayed more ordered secondary structures, a higher solubility, and better gelling properties after the HIU treatment, especially at 20 min. The results of this study will be beneficial to the scientific community as well as industrial applications. 


\subsection{Introduction}

High-intensity ultrasound (HIU), which has frequencies in the range of $20-100 \mathrm{kHz}$ and power in the range of $10-1000 \mathrm{~W} / \mathrm{cm}^{2}$, has drawn considerable attention recently as a promising nonthermal processing method with little impact on the environment [90]. Due to cavitation, shear stresses, dynamic agitation, and turbulence, as well as the promotion of some chemical reactions, HIU has the ability to alter the properties of biopolymers physically or chemically [91]. The ultrasonic treatment of soy protein has been studied in the last decade since soy protein is one of the most promising plant proteins in the food industry. For example, Jambrak et al. found that commercial soy protein isolate (SPI) and soy protein concentrate (SPC) showed a smaller particle size but higher solubility and apparent viscosity after sonication [92]. Arzeni et al. found that HIU could reduce the viscosity and particle size of commercial SPI [93]. Upon their treatment, the free sulfhydryl groups remained unchanged. On the other hand, Hu et al. reported that the HIU treatment increased the free sulfhydryl content of commercial SPI as well as its solubility and surface hydrophobicity [94]. Besides this, HIU could also induced the dissociation and/or aggregation of protein molecules. Zheng et al. found that HIU could increase aggregation in native SPI while dissociating the aggregates in alcohol-denatured and heat moisture-denatured SPI [95].

The divergent results in the literature reveal that the effects of implementing HIU treatment on soy protein depend on not only the treatment conditions but also the intrinsic properties of the protein. Most of the previous studies were conducted with total protein isolates or concentrates, which limited the insight into the phenomena responsible for the observed changes due to the complexity of the protein composition. Like many food proteins, soy protein is a multicomponent biomacromolecule mixture which consists of $\beta$-conglycinin and glycinin as the two main components. They are the vital intrinsic factors that affect the physicochemical properties, structural characteristics, and aggregation behaviors of soy protein. It has been found that, compared with isolated $\beta$-conglycinin, HIU had minor effects on isolated glycinin at pH 7.0 [96], and the effects of HIU on the isolated glycinin varied with different ionic strengths [91]. Therefore, how these soy protein components, especially glycinin, influence the HIU treatment of soy protein is worth further study.

When it comes to the enzymolysis of protein, the most common method is limited proteolysis. Through the degree of hydrolysis (DH), limited proteolysis allows researchers to obtain reproducible results and avoid excessive protein hydrolysis [97]. However, since $\mathrm{DH}$ only indicates the proportion of cleaved peptide bonds, when the substrate is a multicomponent protein like soy protein, limited proteolysis can hardly control and determine which specific component or subunit of protein is being hydrolyzed. As a result, a novel method called selective proteolysis has been studied in recent years [39,98-100]. 
Differently from traditional proteolysis, selective proteolysis is more precise and controllable in terms of the acting sites, by which one can selectively and exclusively degrade a target component in soy protein without affecting the others. In general, glycinin has a more compact globular conformation and lower molecular flexibility than $\beta$-conglycinin, which often limits the functional properties of soy protein. $\mathrm{Li}$ et al. found that selectively hydrolyzing glycinin could facilitate soy protein to form interfacial films with high viscoelastic moduli, and thus its emulsifying properties improved $[98,99]$. Our previous study found that decreasing the $\beta$-conglycinin component by selective proteolysis was beneficial to the formation of longer fibrils in the subsequent protein fibrillation process [100].

To the best of our knowledge, few studies have investigated the effects of HIU on protein hydrolysates, let alone the hydrolysates prepared by selective hydrolysis. Against the abovementioned background, selective proteolysis was used in this study to alter the composition of a soy protein substrate to obtain a soy protein hydrolysate in which the glycinin component was selectively degraded (referred to as degraded glycinin hydrolysate, DGH). Then, the DGH was treated by HIU $(20 \mathrm{kHz}, 400 \mathrm{~W})$ for different times $(0,5,20$, and 40 min), together with a control SPI sample (referred to as CSPI, blank group), which was prepared similarly except for adding protease. By comparing the changes in their physical, structural, and aggregation properties induced by HIU processing, this study for the first time reveals how selectively hydrolyzing glycinin affects the HIU treatment of soy protein, which will be beneficial to the scientific community as well as the industrial application of vegan food based on soy protein hydrolysates.

\subsection{Materials and Methods}

\subsubsection{Materials}

Cold defatted soy flour (protein concentration 56\% $(N \times 6.25)$ (dry based) was obtained from Qi Tian Biotechnology Co., Ltd. (Henan, China). A Lowry assay kit was purchased from Labaide Biosciences Co. (Shanghai, China). Papain (21 units/mg), antipain (Sigma nr A6191), and 1-anilino-8-naphthalene-sulfonate (ANS) were purchased from Sigma-Aldrich Co. (St. Louis, MO, USA). Deionized distilled water (DDW) was prepared by a Milli-Q Direct 8 water purification system (Merck Millipore Co., Burlington, NJ, USA). All the other reagents used were of analytical grade.

\subsubsection{Preparation of native soy protein isolate (SPI)}

According to our previous study [100], defatted soy flour was dispersed in DDW $(1: 20 \mathrm{w} / \mathrm{w})$ and adjusted to $\mathrm{pH} 8.0$ with $2 \mathrm{~mol} / \mathrm{L} \mathrm{NaOH}$. The dispersion was stirred at room temperature $\left(25^{\circ} \mathrm{C}\right.$ ) for $1.5 \mathrm{~h}$ before centrifuging at $9000 \mathrm{~g}, 4^{\circ} \mathrm{C}$, for $20 \mathrm{~min}$. The supernatant was 50 
adjusted to pH 4.5 with $2 \mathrm{~mol} / \mathrm{L} \mathrm{HCl}$ and centrifuged $\left(9000 \mathrm{~g}, 4^{\circ} \mathrm{C}\right.$ ) again for $20 \mathrm{~min}$. The obtained SPI precipitate was re-dissolved $(1: 4 \mathrm{w} / \mathrm{w})$ in DDW and adjusted to $\mathrm{pH} 7.0$ before freeze drying. The protein concentration of the obtained SPI was $92.85 \% \pm 0.35 \%(\mathrm{~N} \times$ 6.25) according to the Dumas measurement.

\subsubsection{Selective proteolysis on glycinin component}

The selective proteolysis on the glycinin component of SPI was based on the method of $\mathrm{Li}$ et al. [98]. The lyophilized SPI (25 g) was well dispersed in $500 \mathrm{~mL}$ of DDW and then adjusted to $\mathrm{pH} 2.0$ and $37^{\circ} \mathrm{C}$. Pepsin was added to the SPI dispersion with an E/S ratio of $0.02 \%$, and the enzymatic reaction was carried out by incubating at $\mathrm{pH} 2.0$ and $37^{\circ} \mathrm{C}$ for $40 \mathrm{~min}$. After that, the reaction was terminated by neutralizing the dispersion to $\mathrm{pH} 7.0$ and boiling for $5 \mathrm{~min}$. The hydrolysate was then dialyzed against DDW in molecular porous membrane tubing (MWCO: $0.5 \mathrm{kDa}$; Spectrum Medical Industries Inc., USA) at $4{ }^{\circ} \mathrm{C}$ for 48 $\mathrm{h}$ before freeze drying. This lyophilized sample was referred to as degraded glycinin hydrolysate (DGH). The degree of hydrolysis (DH) of the DGH was $1.87 \% \pm 0.08 \%$ according to the pH-STAT measurement [30]. A control SPI sample (CSPI) was prepared in the same manner (incubated for $40 \mathrm{~min}$ ) without the addition of pepsin.

\subsubsection{High-intensity ultrasound (HIU) treatment of proteins}

The DGH and CSPI dispersions $(10 \%, \mathrm{w} / \mathrm{v})$ were prepared by stirring lyophilized powder into DDW for $1 \mathrm{~h}$. An ultrasound processor (Scientz Biotechnology Co. Ltd., China) with a 0.636 $\mathrm{cm}$ diameter titanium probe was used. The protein dispersions $(25 \mathrm{~mL})$ were sonicated $(20$ $\mathrm{kHz}, 400 \mathrm{~W}$ ) for $0,5,20$, and $40 \mathrm{~min}$ ( $4 \mathrm{~s}: 2 \mathrm{~s}$ on/off cycles) in $50 \mathrm{~mL}$ beakers, which were immersed in an ice-water bath. After sonication, all the samples were lyophilized and stored in airtight containers. The actual power and intensity of the HIU treatment in the present study were measured by the method of Arzeni et al. [93]. DDW ( $25 \mathrm{~mL}$ ) was used for the acoustic power estimation. The $C_{p}$ of water is $4.2 \mathrm{~J} /(\mathrm{g} \cdot \mathrm{K})$. The ultrasound intensity in this research was $34-37 \mathrm{~W} / \mathrm{cm}^{2}\left(0.43-0.47 \mathrm{~W} / \mathrm{cm}^{3}\right)$.

\subsubsection{Sodium dodecyl sulfate-polyacrylamide gel electrophoresis (SDS-PAGE) analysis}

SDS-PAGE was performed based on the method of Laemmli [47], using $12 \%$ separated gel and $5 \%$ stacking gel. The sample solutions $(2 \mathrm{mg} / \mathrm{mL}$ ) were prepared with an SDS-PAGE sample buffer $(0.0625 \mathrm{~mol} / \mathrm{L}$ Tris- $\mathrm{HCl}(\mathrm{pH} 8.0)$ containing $10 \%(\mathrm{v} / \mathrm{v})$ glycerol, $5 \%(\mathrm{v} / \mathrm{v}) 2-$ mercaptoethanol, $2 \%(w / v)$ Sodium dodecyl sulfate (SDS), and $0.25 \%(w / v)$ bromophenol blue). Aliquots $(15 \mu \mathrm{L})$ of the sample solution were loaded per well after incubating at $95{ }^{\circ} \mathrm{C}$ for $5 \mathrm{~min}$. The gel was stained with Coomassie Brilliant Blue R-250 for $1 \mathrm{~h}$ and destained 
with $20 \%$ methanol and $10 \%$ acetic acid mixed solution. The gel was scanned and analyzed by a GS-900 calibrated densitometer with the Image Lab software (Bio-Rad Laboratories, Inc., Hercules, California, USA).

\subsubsection{Determination of protein solubility}

The protein solubility was determined by the method of Huang et al. [101] with modifications. The lyophilized samples were added to the DDW $(10 \mathrm{mg} / \mathrm{mL})$ and stirred for $1 \mathrm{~h}$ at $25^{\circ} \mathrm{C}$. Then, the dispersions were centrifuged at $10,000 \mathrm{~g}$ for $20 \mathrm{~min}$. The protein content in the dispersion and the protein content in the supernatant after centrifugation both were determined by the Lowry method, using bovine serum albumin as standard. The absorbance at $750 \mathrm{~nm}$ was measured by a Bio-spectrophotometer (Eppendorf Co. Ltd., Hamburg, Germany). The protein solubility was calculated as follows:

protein solubility $(\%)=($ protein content of the supernatant)/ (total protein content before centrifugation) $\times 100 \%$.

\subsubsection{Dynamic light scattering (DLS)}

The particle sizes of the samples were determined by dynamic light scattering (DLS) using a Nano-ZS Zetasizer (Malvern Instrument Ltd., Worcestershire, UK) [91]. The sample supernatants mentioned in Section 3.2.6 were diluted to $0.15 \mathrm{mg} / \mathrm{mL}$ with DDW to avoid multiple scattering and then measured in $1 \mathrm{~cm} \times 1 \mathrm{~cm}$ disposable cuvettes (model: DTS0012) at $25^{\circ} \mathrm{C}$. The backscattering angle was $173^{\circ}$, the refractive index (water) was 1.333 , and the equilibration time was $60 \mathrm{~s}$.

\subsubsection{Surface hydrophobicity $\left(\mathrm{H}_{0}\right)$ measurement}

Based on the method of Kato et al. [102], the lyophilized samples were dissolved in 0.01 $\mathrm{mol} / \mathrm{L}$ of phosphate buffer $(\mathrm{pH} 7.0)$ to a series of protein concentrations $(0.05,0.1,0.2,0.5$, 1 , and $2 \mathrm{mg} / \mathrm{mL}$ ). Then, each sample solution ( $5 \mathrm{~mL}$ ) was mixed with $40 \mu \mathrm{L}$ of 1 -anilino-8naphthalene sulfonate (ANS) solution $(8.0 \mathrm{mmol} / \mathrm{L}$ in $0.01 \mathrm{~mol} / \mathrm{L}$ phosphate buffer, $\mathrm{pH} 7.0$ ). The fluorescence intensity (FI) was measured by a F-4600 fluorescence spectrophotometer (Hitachi, Japan) at wavelengths of $390 \mathrm{~nm}$ (excitation) and $470 \mathrm{~nm}$ (emission). The slope of the FI vs. protein concentration plot (calculated by a linear regression analysis) was used as the index of surface hydrophobicity $\left(\mathrm{H}_{0}\right)$. 


\subsubsection{Circular dichroism spectra measurement}

The circular dichroism spectra were collected in the Far-UV range $(190 \sim 250 \mathrm{~nm})$ by a J1500 circular dichroism spectropolarimeter (Jasco Corp., Tokyo, Japan) at $25^{\circ} \mathrm{C}$. The sample supernatants in Section 3.2.6 were diluted to $0.15 \mathrm{mg} / \mathrm{mL}$ and measured in $0.1 \mathrm{~cm}$ quartz $C D$ cuvettes. The scan rate, response, and bandwidth were set as $50 \mathrm{~nm} / \mathrm{min}, 4 \mathrm{~s}$, and 1.0 $\mathrm{nm}$, respectively [95]. The recorded spectra were an average of three scans and corrected by subtracting the spectrum of the protein-free buffer. The proportions of the four secondary structures ( $a$-helix, $\beta$-sheet, $\beta$-turn, random coil) were derived from Yang's equation. A mean value of 110 for the amino acid residue was used in the calculation.

\subsubsection{Intrinsic fluorescence spectra measurement}

The intrinsic fluorescence spectra of the samples were obtained by a F-4600 fluorescence spectrophotometer (Hitachi, Japan) at $25^{\circ} \mathrm{C}$, based on the method of Jiang et al. [103]. The sample supernatants mentioned in Section $\mathbf{3 . 2 . 6}$ were diluted to $0.075 \mathrm{mg} / \mathrm{mL}$ with DDW and then excited at $290 \mathrm{~nm}$. The emission spectra were recorded from 300 to $400 \mathrm{~nm}$. Both the excitation (Ex) slit and emission (Em) slit were set as $5 \mathrm{~nm}$, and the scan speed was $240 \mathrm{~nm} / \mathrm{min}$.

\subsubsection{FT-Raman spectra measurement}

The FT-Raman spectra were collected on lyophilized samples by an INVIA laser Raman spectrometer (Renishaw, UK) under the following settings: laser wavelength, $1064 \mathrm{~nm}$; laser power, $1 \mathrm{~W}$; spectral resolution, $4 \mathrm{~cm}^{-1}$; number of scans, 800 . The obtained spectra were normalized by using a phenylalanine band at around $1004 \mathrm{~cm}^{-1}$ as an internal standard, since its intensity was neither sensitive to the conformational changes nor the microenvironmental changes [104].

\subsubsection{Scanning electron microscopy (SEM)}

The morphology of the lyophilized protein samples was observed by a scanning electron microscope (SU8000, Hitachi, Tokyo, Japan). Before using scanning electron microscopy, the samples were ground by a mortar and pestle and then coated with gold.

\subsubsection{Small-amplitude oscillatory shear (SAOS) tests}

The thermal gelation of the sample dispersion ( $9 \% \mathrm{w} / \mathrm{v}, \mathrm{pH} 7.0)$ was monitored with a MCR302 rheometer (Anton Paar, Austria) with a sandblasted concentric cylinder (CC17) and a solvent trap. The measurement was conducted within the linear viscoelastic region at a 
constant strain of $1 \%$ and a frequency of $1 \mathrm{~Hz}$. The temperature sweep was as follows: heating from $20^{\circ} \mathrm{C}$ to $95^{\circ} \mathrm{C}$ at $3{ }^{\circ} \mathrm{C} / \mathrm{min}$, holding at $95^{\circ} \mathrm{C}$ for $30 \mathrm{~min}$, cooling from $95^{\circ} \mathrm{C}$ to $20^{\circ} \mathrm{C}$ at $3^{\circ} \mathrm{C} / \mathrm{min}$, holding at $20^{\circ} \mathrm{C}$ for $5 \mathrm{~min}$. The storage modulus ( $\left.\mathbf{G}^{\prime}\right)$ and loss modulus (G") were recorded as a function of time.

\subsubsection{Statistical analysis}

All the analytical determinations were performed in triplicate and the results were presented as the means \pm standard deviation. The figures were plotted by the Origin 2018 software (OriginLab, Northampton, Massachusetts, USA). An ANOVA (one-way analysis of variance) and Duncan's test at $p<0.05$ were conducted by SPSS 25.0 (SPSS Inc., USA) to evaluate the statistical significance of the differences among the means.

\subsection{Results and discussion}

\subsubsection{SDS-PAGE}

As shown in Fig. 3.1, under reducing conditions (with 2-mercaptoethanol) the CSPI showed a typical SDS-PAGE profile of soy protein, which mainly consisted of individual subunits of $\beta$-conglycinin and glycinin. As a trimer, $\beta$-conglycinin consisted of three subunits: $a^{\prime}(\sim 80$ $\mathrm{kDa})$, a $(\sim 70 \mathrm{kDa})$, and $\beta(\sim 50 \mathrm{kDa})$. On the other hand, the glycinin component is a hexamer which contained an acidic subunit $\left(\mathrm{A}_{1-4}\right)(32-40 \mathrm{kDa})$ and a basic subunit $(B)(\sim 20$ $\mathrm{kDa})$. It was clear that the bands for the acidic subunit and the basic subunit all disappeared in DGH, while the bands for the subunits of $\beta$-conglycinin still existed. In addition, wide and vague bands below each subunit of glycinin appeared in DGH, which represented the small fractions with different molecular weights that derived from the degradation of glycinin. According to the densitometric analysis (Table 3.1), the relative content of all the glycinin subunits decreased dramatically from $60.7 \%$ in CSPI to only $2.5 \%$ in DGH, while the relative content of the small fractions increased significantly from $5.8 \%$ in CSPI to $38.5 \%$ in DGH. Correspondingly, the proportion of all the $\beta$-conglycinin subunits increased from $33.5 \%$ in CSPI to $59.0 \%$ in DGH. Achieving this selectivity relies on the enzymatic reaction conditions as well as the enzyme. On the one hand, glycinin is known to be denatured preferentially in an acidic $\mathrm{pH}$ [105]. Therefore, compared with $\beta$-conglycinin, the glycinin component would be denatured to a much higher extent in the present reaction conditions $\left(\mathrm{pH} 2.0,37^{\circ} \mathrm{C}\right)$ and time (40 min), which made the cleavage sites in glycinin more accessible to the enzyme. On the other hand, pepsin was chosen because it shows selectivity to glycinin and it is optimally active at pH 2.0 [39]. Owing to these two aspects, the glycinin component of SPI was hydrolyzed successfully in the present study, while the $\beta$-conglycinin component was not affected. 


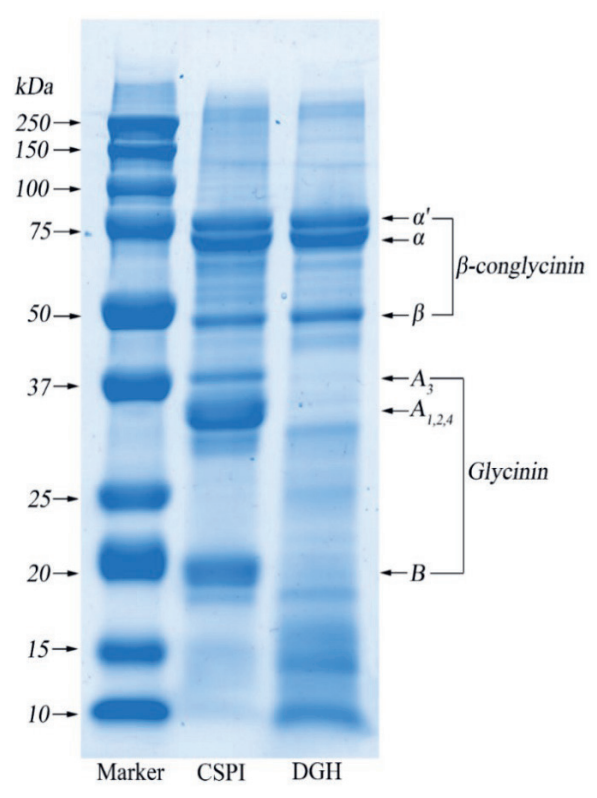

Figure 3.1. SDS-PAGE patterns of control soy protein isolate (CSPI, blank group) and degraded glycinin hydrolysate (DGH, contrast group).

Table 3.1. The relative contents of the individual subunits and small protein fractions in CSPI and DGH according to the densitometric analysis of SDS-PAGE.

\section{CSPI DGH}

\begin{tabular}{lllll}
\hline Band & MW $(\mathrm{kDa})$ & Content $(\%)$ & MW $(\mathrm{kDa})$ & Content $(\%)$ \\
$\mathrm{a}^{\prime}$ & 79.0 & 9.3 & 80.2 & 13.2 \\
$\mathrm{a}$ & 70.4 & 13.6 & 71.5 & 25.7 \\
$\beta$ & 49.5 & 10.6 & 50.4 & 20.1 \\
$\mathrm{~A} 3$ & 39.2 & 7.6 & 38.6 & 0.1 \\
$\mathrm{~A} 1,2,4$ & 33 & 27.6 & 32.5 & 1.9 \\
Small fractions & $32 \sim 22$ & 4.2 & $32 \sim 22$ & 1.2 \\
B & 19.7 & 25.5 & 20.1 & 0.5 \\
Small fractions & $<18.3$ & 1.6 & $<18.3$ & 37.3 \\
\hline
\end{tabular}




\subsubsection{Solubility}

As shown in Fig. 3.2, the solubility of CSPI before the HIU treatment was 34.5\%, which agreed with Huang et al. that the solubility of the commercial SPI after acid treatment $(\mathrm{pH}$ $3.0,1 \mathrm{~h}$ ) was about $30 \%$ at $\mathrm{pH} 7.0$ [101]. The higher solubility $(47.2 \%)$ showed by DGH suggested that selective hydrolysis on glycinin could increase the solubility of soy protein by breaking the peptide bonds and reducing the particle size (Section 3.3.3). HIU increased the solubility of DGH and CSPI significantly $(p<0.05)$ to $84.6 \%$ and $81.0 \%$ after $5 \mathrm{~min}$. This improvement could be explained by two reasons: (1) the HIU treatment could reduce the particle size of proteins, which improved the protein dispersibility in the solvent and the protein-solvent interactions, so the solubility of the protein increased [93]; (2) HIU could induce the transformation of insoluble aggregates or precipitates to soluble protein aggregates, which made more protein in the soluble state [106]. Interestingly, when the HIU treatment was continued to $20 \mathrm{~min}$, the solubility of the CSPI became lower, while the solubility of DGH kept increasing. The solubility of DGH reached $92.5 \%$ at 20 min, which was significantly higher $(p<0.05)$ than that of CSPI $(79.3 \%)$. Since the surface-active and gelation properties require proteins to be soluble in the relevant medium, selectively hydrolyzing glycinin may facilitate the ultrasonic modification of soy protein in terms of these techno-functionalities. When the HIU treatment was prolonged to $40 \mathrm{~min}$, the solubility of both the samples decreased. Similar results were found by Huang et al. [101] and Jiang et al. [103] that the solubility of commercial SPI and black bean protein could be unchanged or decreased with a further increase in ultrasonic time.

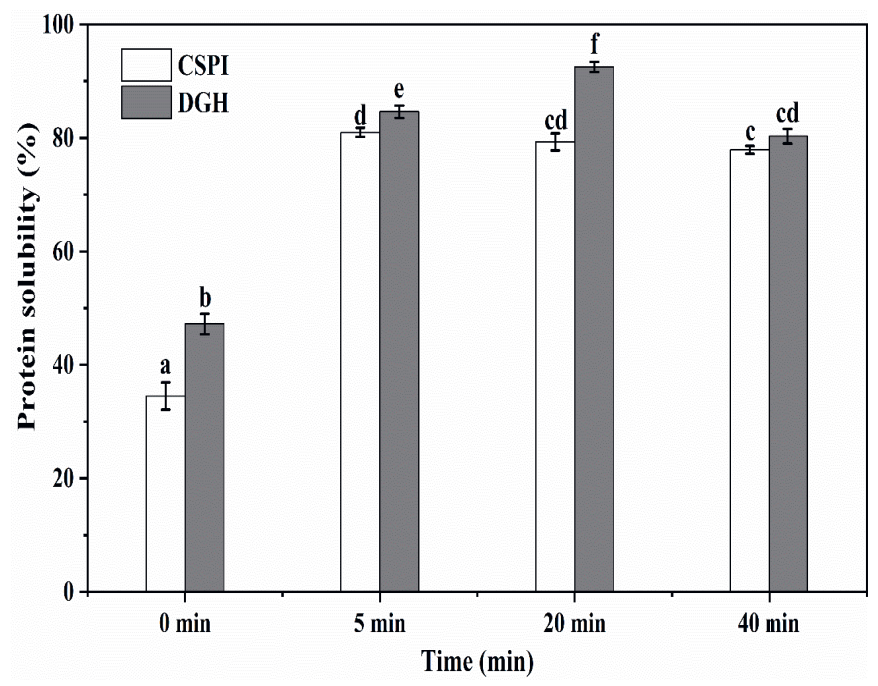

Figure 3.2. Effect of high-intensity ultrasound treatment ( $20 \mathrm{kHz}$ at $400 \mathrm{~W}$ for $0,5,20,40 \mathrm{~min}$ ) on the protein solubility of CSPI and DGH. Different lowercase letters (a, b, c...) indicate significant differences between samples, at $p<0.05$ using Duncan's test. Error bars represent the standard deviations. 


\subsubsection{Particle size}

As shown in Fig. 3.3, before HIU treatment the DGH and CSPI both demonstrated a multimodal particle size distribution (PSD) with three peaks. The hydrodynamic diameter of native SPI has been reported to be around $34 \mathrm{~nm}$ (at pH 7.0 and $25^{\circ} \mathrm{C}$ ) [107], which explains the middle peak at $28-38 \mathrm{~nm}$. The peak located below $10 \mathrm{~nm}$ could be attributed to the small protein fractions that were dissociated from SPI, while the peak at above 100 $\mathrm{nm}$ indicates the existence of large aggregates. Enzymatic hydrolysis shifted all three peaks of DGH to smaller sizes compared to those of CSPI, and DGH showed a lower average size and higher polydispersity index (PDI) (Table 3.2). After the HIU treatment, the PSD of DGH and CSPI both changed into a unimodal distribution and their PDI both decreased, showing the homogenization effects of high-intensity ultrasound. Interestingly, the single peaks of the HIU-treated samples were all located at higher sizes than the peak of the native SPI (the middle one in the multimodal distribution), and their average sizes all increased. This means most of the soluble proteins after HIU treatment, existed in the form of aggregates. Combining the markedly increased solubility (Fig. 3.2), the formation of soluble aggregates after the HIU treatment was mainly responsible for the improved solubility in the present study. Tang et al. reported that the high-frequency oscillation caused by HIU (200 W, 15 $\mathrm{kHz}$ ) could slow down the association of small unstable aggregates in soy protein dispersion while promoting the interactions between unstable aggregates and other soluble protein components (e.g., the $a$ and $a^{\prime}$ subunits of $\beta$-conglycinin), eventually forming soluble aggregates with a relatively stable structure [106].

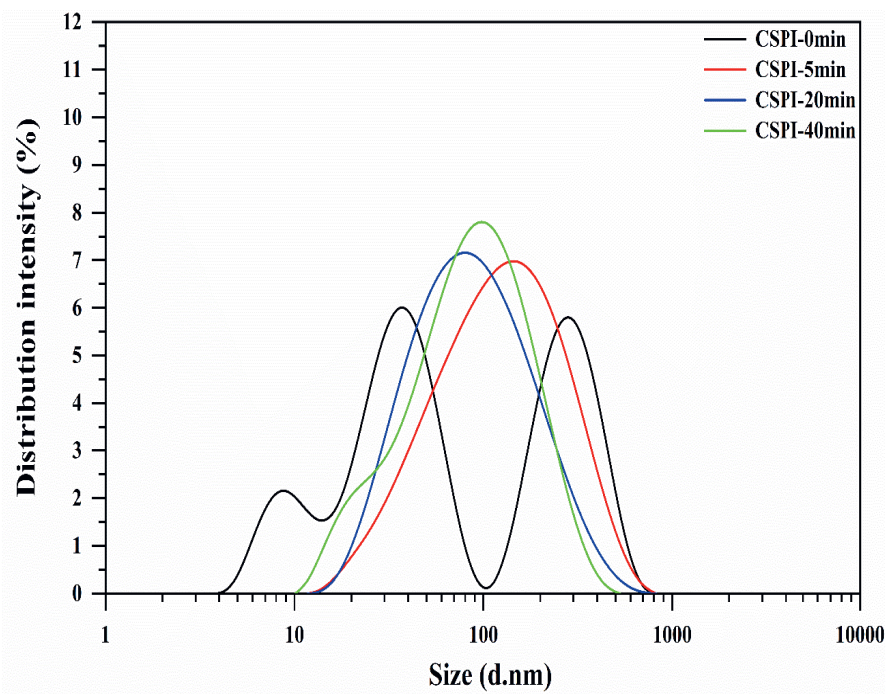

Figure 3.3. (A) 


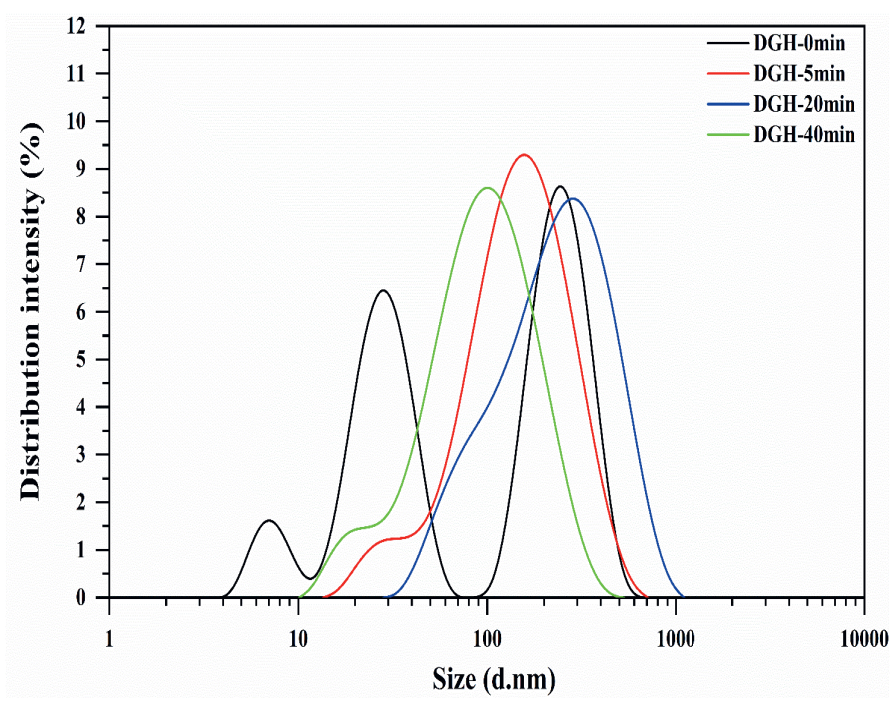

(B)

Figure 3.3. Effect of high-intensity ultrasound treatment ( $20 \mathrm{kHz}$ at $400 \mathrm{~W}$ for $0,5,20,40 \mathrm{~min}$ ) on the particle size distribution of CSPI and DGH.

Table 3.2. Effect of high-intensity ultrasound treatment ( $20 \mathrm{kHz}$ at $400 \mathrm{~W}$ for $0,5,20,40 \mathrm{~min}$ ) on the particle size distribution of CSPI (A) and DGH (B). Values are means \pm standard deviation. Different lowercase letters $(a, b, c, d)$ indicate significant differences ( $p<0.05$, Duncan's test) between high-intensity ultrasound HIU times in the same column.

\begin{tabular}{|c|c|c|c|c|}
\hline \multirow{2}{*}{ Sample } & \multicolumn{2}{|l|}{ CSPI } & \multicolumn{2}{|l|}{$\mathrm{DGH}$} \\
\hline & Z-average $(\mathrm{nm})$ & PDI & Z-average $(\mathrm{nm})$ & PDI \\
\hline $0 \mathrm{~min}$ & $66.09 \pm 4.93^{a}$ & $0.428 \pm 0.040^{b}$ & $48.90 \pm 0.45^{a}$ & $0.897 \pm 0.108^{b}$ \\
\hline $5 \mathrm{~min}$ & $90.52 \pm 1.13^{c}$ & $0.341 \pm 0.033^{a}$ & $119.57 \pm 1.36^{c}$ & $0.321 \pm 0.038^{a}$ \\
\hline $20 \min$ & $72.10 \pm 0.03^{b}$ & $0.382 \pm 0.012^{a b}$ & $131.90 \pm 1.64^{d}$ & $0.308 \pm 0.010^{a}$ \\
\hline $40 \min$ & $67.42 \pm 0.19 \mathrm{ab}$ & $0.374 \pm 0.012^{a}$ & $75.29 \pm 1.59^{b}$ & $0.412 \pm 0.028^{a}$ \\
\hline
\end{tabular}


When the HIU treatment was prolonged ( $>5 \mathrm{~min}$ ), the average size of CSPI showed a decreasing trend. In contrast, the PSD of DGH further moved to larger sizes, with the peak centered at $295.0 \mathrm{~nm}$ at $20 \mathrm{~min}$ (Fig. 3.3), and its average size decreased only after $40 \mathrm{~min}$ (Table 3.2). These trends displayed here were consistent with the trends shown in the solubility (Section 3.3.2). These phenomena reflected that HIU could induce the formation of soluble aggregates in both samples within a certain time. However, the longer duration of HIU processing would not promote further aggregation but dissociate those newly formed aggregates. Zheng et al. observed that HIU $\left(80 \mathrm{~W} / \mathrm{cm}^{2}\right)$ increased the particle size of native SPI aggregates after $10 \mathrm{~min}$ while reducing the particle size after $25 \mathrm{~min}$ [95]. Similarly, Shanmugam et al. found that soluble whey/casein aggregates and micellar aggregates were formed during the first 30 min of sonication $\left(0.31\right.$ and $\left.0.63 \mathrm{~W} / \mathrm{cm}^{3}\right)$, but prolonged sonication caused the partial disruption of whey proteins from these aggregates due to the continuous shear [108]. Noticeably, the soluble aggregates in DGH continued aggregating for a relatively longer duration, and DGH nanoparticles existed as bigger aggregates with relatively uniform sizes (Table 3.2). It has been reported that glycinin tended to form insoluble aggregates, while $\beta$-conglycinin tended to form soluble aggregates, but the subunits from glycinin could interact with $\beta$-conglycinin to form soluble aggregates as well $[2,109,110]$. As shown in the SDS-PAGE (Fig. 3.1), selective proteolysis in this work retained the $\beta$-conglycinin while disintegrating glycinin into small fractions, which may provide DGH with more "building blocks" for the soluble aggregation in the subsequent HIU treatment.

\subsubsection{Surface hydrophobicity}

As shown in Fig. 3.4, the surface hydrophobicity $\left(\mathbf{H}_{\mathbf{0}}\right)$ of DGH was higher than that of CSPI before the HIU treatment. This was expected, since the enzymatic cleavage destroyed the compact structure of glycinin, which made more hydrophobic groups accessible to external fluorescence probes-i.e., ANS-thus showing a higher $\mathrm{H}_{0}$. HIU increased the $\mathrm{H}_{0}$ of DGH and CSPI significantly $(p<0.05)$, although the rate of increase tended to slow down with treatment time. By the cavitation effect of HIU, a great extent of molecular unfolding and structural changes could happen in protein molecules, which enhanced their surface hydrophobicity and promoted intermolecular aggregation [111]. Interestingly, although the $\mathrm{H}_{0}$ of both the samples increased, the $\mathrm{H}_{0}$ of DGH became lower than that of CSPI after the HIU treatment. Hydrophobic interactions have been viewed as the driving force for protein aggregation [112]; the lower $\mathrm{H}_{0}$ suggested that, in the simultaneous effects of HIU-induced denaturation and aggregation, more exposed hydrophobic groups in DGH participated in the hydrophobic interactions during the formation of soluble aggregates. As reported previously $[113,114]$, partially unfolded proteins with an initially higher surface hydrophobicity could cause more extensive bonding and aggregation, which in turn entrapped the hydrophobic regions and manifested in a lower $\mathrm{H}_{0}$ in $\mathrm{DGH}$. Furthermore, with more intermolecular 
hydrophobic interactions being involved, soluble aggregates in DGH should have higher stability, which could help them to endure a relatively long time of ultrasonic vibration and grow into a bigger size, which agreed with the observation in Section 3.3.3.

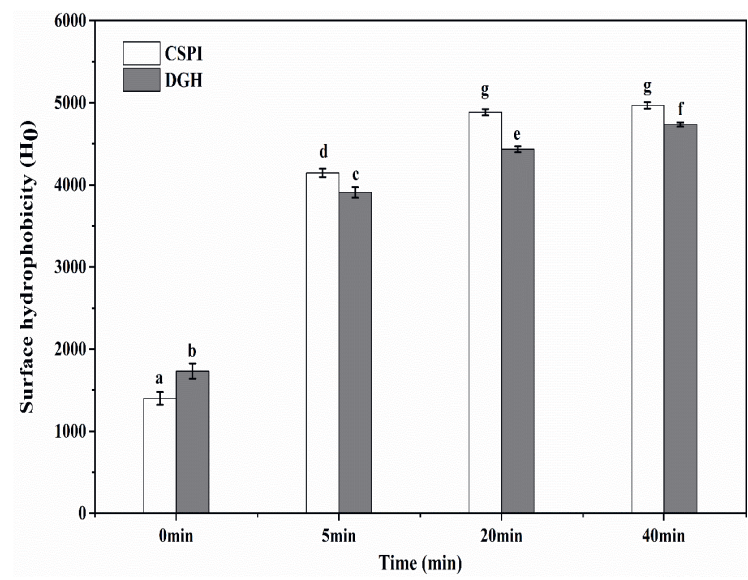

Figure 3.4. Effect of high-intensity ultrasound treatment ( $20 \mathrm{kHz}$ at $400 \mathrm{~W}$ for $0,5,20,40 \mathrm{~min}$ ) on the surface hydrophobicity of CSPI and DGH. Different lowercase letters $(a, b, c \ldots)$ indicate significant differences between samples at $p<0.05$ using Duncan's test. Error bars represent the standard deviations.

\subsubsection{Circular dichroism (CD) spectroscopy}

Table 3.3 shows the secondary structure composition of DGH and CSPI that was deduced from circular dichroism spectroscopy. Prior to the HIU treatment, DGH showed less ordered structure ( $a$-helix and $\beta$-sheet) and more unordered structure ( $\beta$-turn and random coil) than CSPI. HIU induced a significant increase in the a-helix structure, a slight increase in the $\beta$ turn structure, and a decrease in the random coil structure $(p<0.05)$. For the $\beta$-sheet structure, the opposite changes were observed: the $\beta$-sheet structure of DGH increased while that of CSPI decreased. As a result, the composition of the ordered structure in DGH turned out to be higher than that of CSPI, especially at $20 \mathrm{~min}$. The highly repetitive feature of $\beta$-sheet and higher content of ordered structure could be closely correlated with the higher stability of DGH soluble aggregates. Zheng et al. [95] and Hu et al. [94] both observed an increased a-helix content and decreased $\beta$-sheet content in the HIU treatment of native SPI $\left(80 \mathrm{~W} / \mathrm{cm}^{2}\right)$ and commercial SPI $\left(105-138 \mathrm{~W} / \mathrm{cm}^{2}\right)$, which was consistent with the present results of CSPI. However, when the isolated $\beta$-conglycinin and glycinin were solely subjected to an equivalent ultrasonication $\left(105-110 \mathrm{~W} / \mathrm{cm}^{2}\right)$, neither of them showed significant changes in their secondary structures [96]. These results demonstrated that when glycinin existed as a component in soy protein, its influence on the ultrasonic modification of whole protein may not be deduced by simply imposing the same treatment on the isolated glycinin. 
Table 3.3. Effect of high-intensity ultrasound treatment ( $20 \mathrm{kHz}$ at $400 \mathrm{~W}$ for $0,5,20,40 \mathrm{~min}$ ) on the secondary structure of CSPI and DGH. Values are means \pm standard deviation. Different lowercase letters $(a, b, c, d)$ indicate significant differences ( $p<0.05$, Duncan's test) between HIU times in the same row.

\begin{tabular}{|c|c|c|c|c|}
\hline $\begin{array}{l}\text { Composition } \\
(\%)\end{array}$ & CSPI-0 min & CSPI-5 min & CSPI-20 min & CSPI-40 min \\
\hline a-helix & $0.83 \pm 0.25^{a}$ & $8.18 \pm 0.39^{b}$ & $9.10 \pm 0.14^{c}$ & $9.03 \pm 0.07^{c}$ \\
\hline$\beta$-sheet & $66.43 \pm 0.29^{c}$ & $61.20 \pm 0.87^{b}$ & $57.35 \pm 0.49^{a}$ & $59.60 \pm 1.77^{b}$ \\
\hline$\beta$-turn & $0.00 \pm 0.00^{a}$ & $1.08 \pm 0.38 \mathrm{ab}$ & $3.35 \pm 0.78^{c}$ & $1.80 \pm 0.92 b$ \\
\hline Random coil & $32.74 \pm 0.53^{b}$ & $29.54 \pm 0.28^{a}$ & $30.20 \pm 0.14^{a}$ & $29.57 \pm 0.78^{a}$ \\
\hline Ordered & $67.26 \pm 0.53 a b$ & $69.38 \pm 0.65^{c}$ & $66.45 \pm 0.64^{a}$ & $68.63 \pm 1.70 b c$ \\
\hline \multirow[t]{2}{*}{ Unordered } & $32.74 \pm 0.53 b c$ & $30.62 \pm 0.65^{a}$ & $33.55 \pm 0.64^{c}$ & $31.37 \pm 1.70 a b$ \\
\hline & $\mathrm{DGH}-0 \mathrm{~min}$ & DGH-5 min & DGH-20 min & $\mathrm{DGH}-40 \mathrm{~min}$ \\
\hline a-helix & $0.33 \pm 0.21^{a}$ & $7.19 \pm 0.24^{b}$ & $6.83 \pm 0.23^{b}$ & $7.40 \pm 0.44^{b}$ \\
\hline$\beta$-sheet & $60.23 \pm 0.57^{a}$ & $63.00 \pm 0.68^{b}$ & $64.90 \pm 0.72 c$ & $63.67 \pm 0.87 b c$ \\
\hline$\beta$-turn & $0.00 \pm 0.00^{a}$ & $0.15 \pm 0.12 \mathrm{ab}$ & $0.23 \pm 0.14^{a b}$ & $0.50 \pm 0.46^{b}$ \\
\hline Random coil & $39.44 \pm 0.50^{c}$ & $29.66 \pm 0.34 b$ & $28.04 \pm 0.59^{a}$ & $28.43 \pm 0.06^{a}$ \\
\hline Ordered & $60.56 \pm 0.50^{a}$ & $70.19 \pm 0.44^{b}$ & $71.73 \pm 0.58^{c}$ & $71.07 \pm 0.50 \mathrm{bc}$ \\
\hline Unordered & $39.44 \pm 0.50^{c}$ & $29.81 \pm 0.44^{b}$ & $28.27 \pm 0.58^{a}$ & $28.93 \pm 0.50 a b$ \\
\hline
\end{tabular}

\subsubsection{Intrinsic fluorescence spectroscopy}

The intrinsic fluorescence spectra of protein are mainly attributed to Tryptophan (Trp) residues, which can be used for analyzing the tertiary structure. As shown in Fig. 3.5, the intrinsic fluorescence spectra of DGH and CSPI showed a significant blue-shift (decrease in $\left.\lambda_{\max }\right)$ and increased FI after the HIU treatment. The blue-shift of $\lambda_{\max }$ indicated that the microenvironment of Trp residues became less polar. Protein molecular aggregation prevented the interaction of chromophores with the quenching agent present in the solvent and hence a higher FI [115]. DGH displayed a lower $\lambda_{\max }$ and higher FI (except at $40 \mathrm{~min}$ ) than CSPI after the HIU treatment (Table 3.4), which was consistent with the previous 
analysis that showed the soluble aggregation in DGH involved more hydrophobic interactions, which provided the Trp residues with a more hydrophobic microenvironment. At a later stage of the HIU treatment (> $20 \mathrm{~min}$ ), there were no significant changes in the $\lambda_{\max }$ of DGH and CSPI, whereas the FI of them both significantly declined $(p<0.05)$. Similar results were observed by Zhu et al., who showed that the FI of walnut protein decreased with increasing sonication time while the $\lambda_{\max }$ was unchanged, which was an indication of changes in the protein structure and/or aggregation state [116].
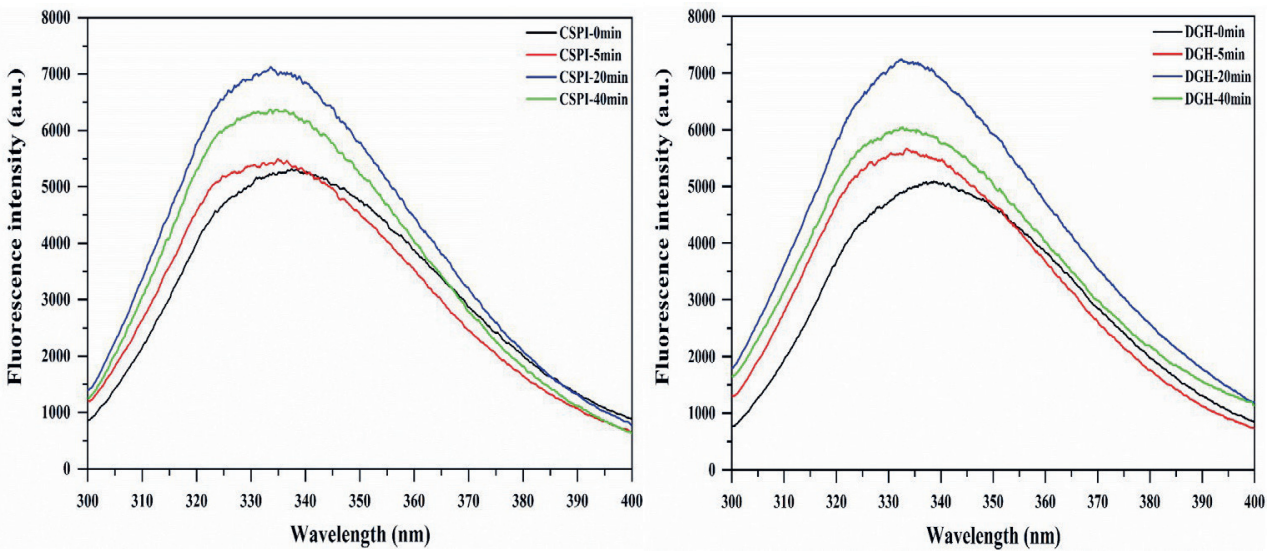

Figure 3.5. Effect of high-intensity ultrasound treatment ( $20 \mathrm{kHz}$ at $400 \mathrm{~W}$ for $0,5,20,40 \mathrm{~min}$ ) on the intrinsic fluorescence emission spectra of CSPI and DGH.

Table 3.4. Effect of high-intensity ultrasound treatment ( $20 \mathrm{kHz}$ at $400 \mathrm{~W}$ for 0, 5, 20, $40 \mathrm{~min}$ ) on the $\lambda_{\max }$ and fluorescence intensity (FI) of CSPI and DGH. Intensity values are means \pm standard deviation. Different lowercase letters $(a, b, c \ldots)$ indicate significant differences $(p<0.05$, Duncan's test) between the fluorescence intensity of different samples.

\begin{tabular}{lllll}
\hline Sample & \multicolumn{3}{l}{ CSPI } & DGH \\
\hline & $\lambda_{\max }(\mathrm{nm})$ & FI (a.u.) & $\lambda_{\max }(\mathrm{nm})$ & FI (a.u.) \\
\hline 0 min & 337.4 & $5311.7 \pm 10.6^{\mathrm{b}}$ & 338.6 & $5085.3^{2} \pm 24.0^{\mathrm{a}}$ \\
$5 \mathrm{~min}$ & 335.0 & $5493.3 \pm 46.7^{\mathrm{c}}$ & 333.4 & $5668.7 \pm 18.4^{\mathrm{d}}$ \\
& & & & \\
$20 \mathrm{~min}$ & 333.6 & $7126.0 \pm 31.1^{\mathrm{g}}$ & 332.4 & $7244.0 \pm 32.5^{\mathrm{h}}$ \\
$40 \mathrm{~min}$ & 333.6 & $6371.7 \pm 53.0^{\mathrm{f}}$ & 332.6 & $6040.7 \pm 15.5^{\mathrm{e}}$ \\
& & & & \\
\hline
\end{tabular}




\subsubsection{FT-Raman spectroscopy}

Disulfide exchange reactions and the formation of disulfide bonds (S-S) play crucial roles in the unfolding and functional aggregation of proteins [117]. The characteristic frequencies of S-S stretching vibrations in the Raman spectra are assigned to gauche-gauche-gauche ( $\mathbf{g}$ g-g, 500-510 cm-1), gauche-gauche-trans (g-g-t, 515-530 cm-1), and trans-gauchetrans (t-g-t, 535-545 cm-1), which are three conformation forms. As shown in Fig. 3.6 and Table 3.5, the Raman peak at 500-510 cm-1 exhibited the highest intensity before the HIU treatment. This is consistent with a previous study, which showed that the S-S stretching vibrations of soy protein tended to be dominated by the g-g-g conformation, since it had the lowest potential energy [104]. However, the intensity of the g-g-g conformation decreased after the HIU treatment, while that of $\mathrm{g}-\mathrm{g}$ - $\mathrm{t}$ and $\mathrm{t}-\mathrm{g}$ - $\mathrm{t}$ conformations both increased $(p<$ 0.05). It has been reported that the g-g-g conformation of disulfide bonds was negatively correlated with the surface hydrophobicity of protein, while the g-g-t conformation promoted the surface hydrophobicity [118], which was in conformity with the increased $\mathrm{H}_{0}$ after the HIU treatment (Section 3.3.4). For DGH, the intensity of the g-g-t and $\mathrm{t}-\mathrm{g}-\mathrm{t}$ conformations increased gradually in the first $20 \mathrm{~min}$ and then decreased after $40 \mathrm{~min}$. For CSPI, significant changes $(p<0.05)$ in the S-S stretching vibrations were only observed in the first 5 min. These phenomena suggested that HIU had a more pronounced effect on the S-S stretching vibration in DGH. Each pair of acidic subunit and basic subunit in glycinin is associated with disulphide bridges [106]; selectively hydrolyzing glycinin could break these disulphide bonds and release sulfhydryl groups. Under the following ultrasonic cavitation effects, the highly reactive free radicals generated from water molecules could oxidize susceptible sulfhydryl groups to form intermolecular disulphide bonds, which might play an important role in the soluble aggregation of DGH $[106,119,120]$. Similarly, Lee et al. found that pH shifting could cause the cleavage of disulphide bonds between glycinin subunits, which led to increased S$\mathrm{S}$ bonds and higher protein solubility in the subsequent ultrasonication [117].

The intensity ratio of 850 to $830 \mathrm{~cm}^{-1}\left(\mathbf{I}_{\mathbf{8 5 0 / 8 3 0}}\right)$ and the intensities of the Raman peaks near 1450 and $2935 \mathrm{~cm}^{-1}$ were used to reflect the microenvironment of Tyr residues and aliphatic amino acid residues, respectively $[104,121]$. HIU first reduced the $\mathrm{I}_{850 / 830}, \mathrm{I}_{1450}$, and $\mathrm{I}_{2935}$ in a short period, indicating that the microenvironment of these residues became less polar due to the protein intermolecular interactions [122]. However, as the HIU treatment was prolonged, the value of these indexes increased again, which indicated that some of the HIU-induced aggregates were disassociated under prolonged ultrasonic shear forces and turbulence, and the amino acid residues that were buried by them became exposed again. However, due to the higher stability of the aggregates, this dissociation phenomenon was obviously delayed in DGH, as these indexes only increased after $40 \mathrm{~min}$. 


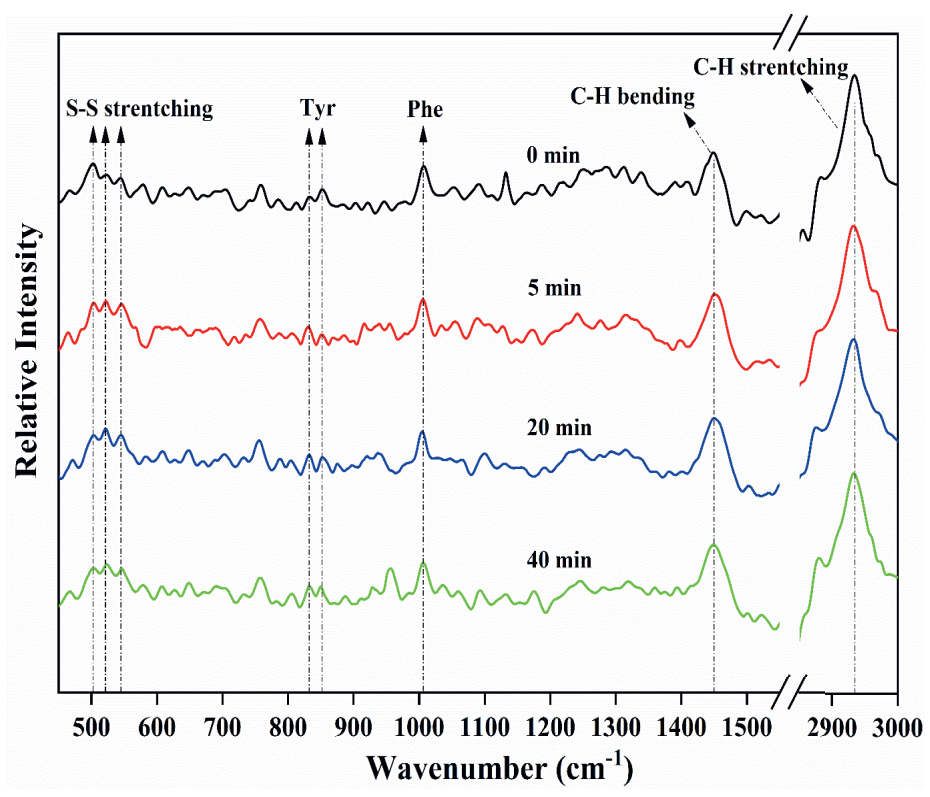

(A)

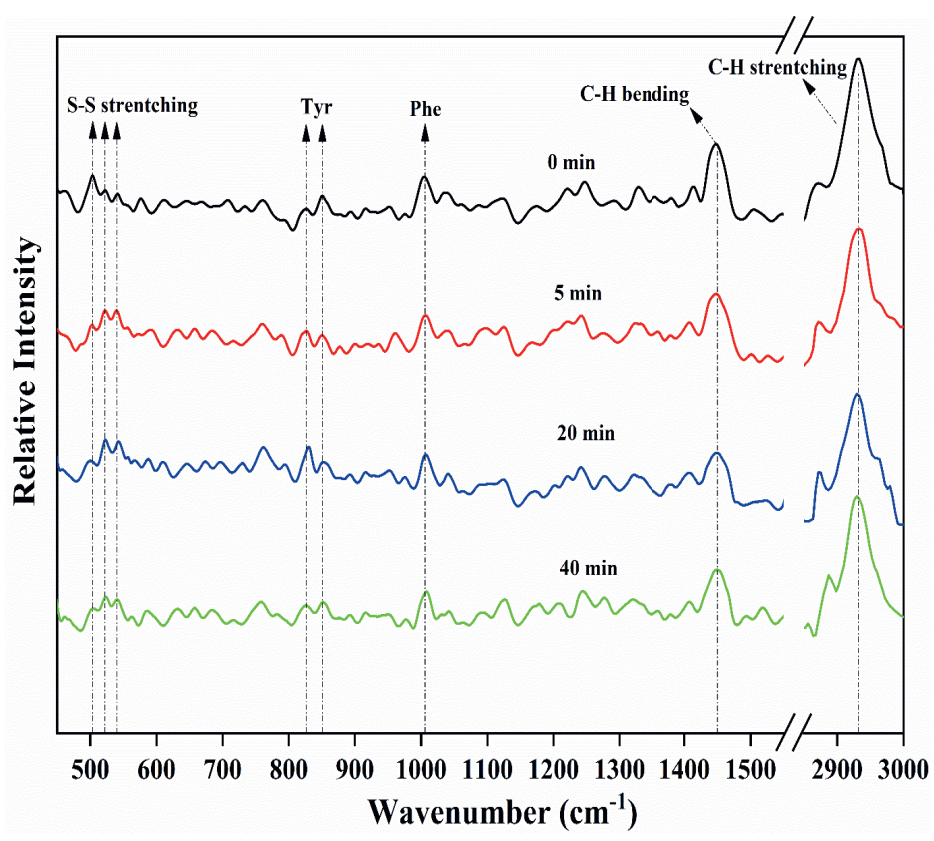

(B)

Figure 3.6. Effect of high-intensity ultrasound treatment $(20 \mathrm{kHz}$ at $400 \mathrm{~W}$ for $0,5,20,40 \mathrm{~min}$ ) on the FTRaman spectra of CSPI (A) and DGH (B) 
Table 3.5. Normalized intensity values at selected regions of the FT-Raman spectra of high-intensity ultrasound (HIU; $20 \mathrm{kHz}$ at $400 \mathrm{~W}$ for 0, 5, 20, $40 \mathrm{~min}$ ) treated CSPI and DGH. Values are means \pm standard deviation. Different lowercase letters ( $a, b, c, d)$ indicate significant differences ( $p<0.05$, Duncan's test) between the HIU times. $\mathrm{g}-\mathrm{g}-\mathrm{g}$ is short for gauche-gauche-gauche, g-g-t is short for gauche-gauche-trans, $t$ $\mathrm{g}-\mathrm{t}$ is short for trans-gauche-trans.

\begin{tabular}{|c|c|c|c|c|}
\hline $\begin{array}{l}\text { Band Assignment } \\
{\left[\text { Wavenumber }\left(\mathrm{cm}^{-1}\right)\right]}\end{array}$ & CSPI-0 min & CSPI-5 min & CSPI-20 min & CSPI-40 min \\
\hline $\begin{array}{l}\text { S-S stretching }(g-g-g) \\
{[500-510]}\end{array}$ & $1.02 \pm 0.02 b$ & $0.97 \pm 0.01^{a}$ & $0.97 \pm 0.02$ & $0.95 \pm 0.03^{a}$ \\
\hline $\begin{array}{l}\text { S-S stretching }(g-g-t) \\
{[515-530]}\end{array}$ & $0.93 \pm 0.02$ & $0.99 \pm 0.02^{b}$ & $1.01 \pm 0.03^{b}$ & $0.99 \pm 0.01 b$ \\
\hline $\begin{array}{l}\text { S-S stretching }(t-g-t) \\
{[535-545]}\end{array}$ & $0.91 \pm 0.01^{a}$ & $0.97 \pm 0.01^{b}$ & $0.96 \pm 0.02 b$ & $0.97 \pm 0.03^{b}$ \\
\hline Tyrosine doublet [850/830] & $1.07 \pm 0.02 \mathrm{~d}$ & $0.93 \pm 0.02 \mathrm{a}$ & $0.97 \pm 0.01^{b}$ & $1.01 \pm 0.03^{c}$ \\
\hline $\mathrm{C}-\mathrm{H}_{2}$ bending [1448-1452] & $1.11 \pm 0.03^{b}$ & $1.03 \pm 0.02^{a}$ & $1.09 \pm 0.03^{b}$ & $1.13 \pm 0.04^{b}$ \\
\hline C-H stretching [2929-2937] & $1.70 \pm 0.04^{b}$ & $1.55 \pm 0.05^{a}$ & $1.69 \pm 0.04^{b}$ & $1.68 \pm 0.03^{b}$ \\
\hline $\begin{array}{l}\text { Band Assignment } \\
{\left[\text { Wavenumber }\left(\mathrm{cm}^{-1}\right)\right]}\end{array}$ & DGH-0 min & DGH-5 min & DGH-20 min & $\mathrm{DGH}-40 \mathrm{~min}$ \\
\hline $\begin{array}{l}\text { S-S stretching }(g-g-g) \\
{[500-510]}\end{array}$ & $1.03 \pm 0.04^{c}$ & $0.94 \pm 0.01 \mathrm{ab}$ & $0.96 \pm 0.02^{b}$ & $0.90 \pm 0.03^{a}$ \\
\hline $\begin{array}{l}\text { S-S stretching }(g-g-t) \\
{[515-530]}\end{array}$ & $0.91 \pm 0.02^{a}$ & $1.05 \pm 0.02 b$ & $1.13 \pm 0.05^{c}$ & $0.99 \pm 0.04 b$ \\
\hline $\begin{array}{l}\text { S-S stretching }(\mathrm{t}-\mathrm{g}-\mathrm{t}) \\
{[535-545]}\end{array}$ & $0.88 \pm 0.01^{a}$ & $1.04 \pm 0.03^{c}$ & $1.11 \pm 0.04^{d}$ & $0.96 \pm 0.04^{b}$ \\
\hline Tyrosine doublet [850/830] & $1.14 \pm 0.04^{c}$ & $0.96 \pm 0.02^{b}$ & $0.88 \pm 0.02^{a}$ & $1.03 \pm 0.06^{b}$ \\
\hline $\mathrm{C}-\mathrm{H}_{2}$ bending $[1448-1452]$ & $1.23 \pm 0.05^{c}$ & $1.16 \pm 0.03^{b}$ & $1.01 \pm 0.02^{a}$ & $1.17 \pm 0.02 \mathrm{bc}$ \\
\hline C-H stretching [2929-2937] & $1.84 \pm 0.08^{c}$ & $1.62 \pm 0.05^{b}$ & $1.44 \pm 0.03^{a}$ & $1.70 \pm 0.06^{b}$ \\
\hline
\end{tabular}

\subsubsection{Scanning electron microscopy (SEM)}

The changes in the microstructure of the lyophilized DGH and CSPI after the HIU treatment were observed by SEM. Fig. 3.7 shows that all the samples presented in the form of massive chunks after freeze drying, but with different shapes and surface morphologies. As previously reported, larger protein aggregates with a layer block structure were observed in sonicated legume proteins in their lyophilized state [94,123]. Before the HIU treatment, DGH displayed a complicated structure with a rough surface, which had many irregular 
humps and cavities of different sizes. On the other hand, CSPI showed a relatively flat structure. This provided tangible evidence of the degradation effects of selective proteolysis on the structural integrity of soy protein. The surface morphology of both samples became flatter and more compact in the early stage of the HIU treatment. Interestingly, compared to CSPI, DGH showed a crystalline microstructure with a smoother surface, only having some debris, especially at $20 \mathrm{~min}$. This denser microstructure of DGH should be ascribed to the smaller inhomogeneities and a larger extent of intermolecular interactions and soluble aggregation in the protein dispersion before freeze drying.
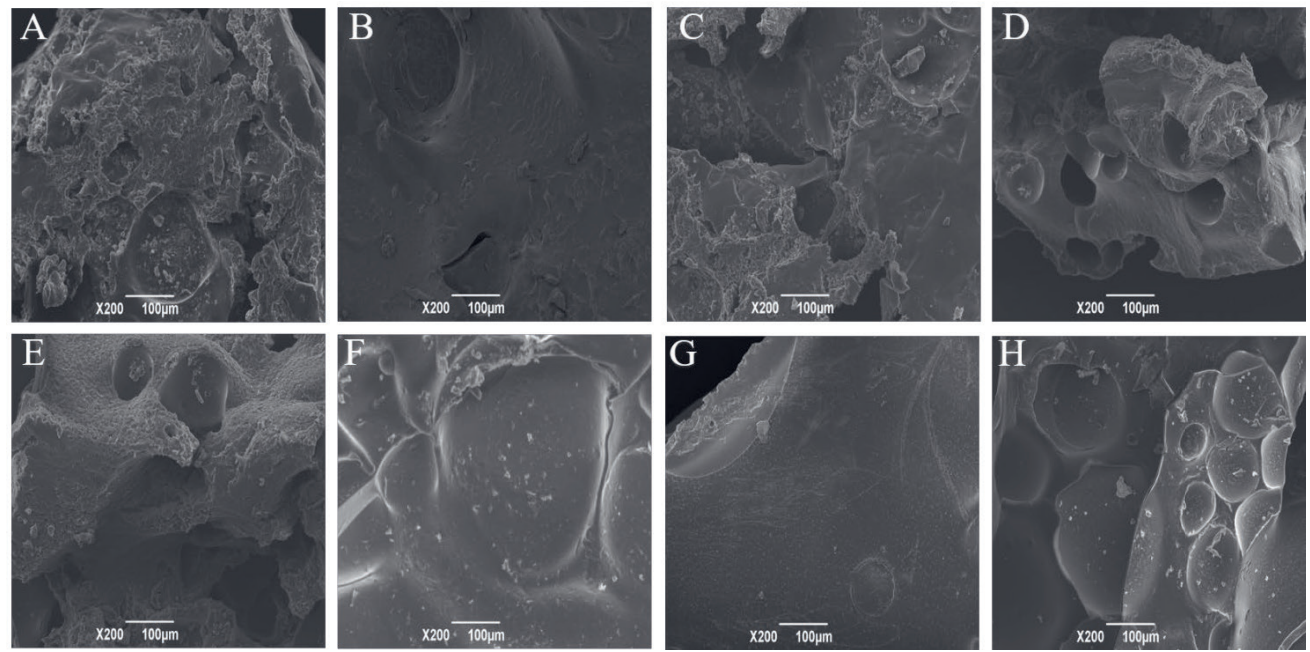

Figure 3.7. Effect of high-intensity ultrasound treatment ( $20 \mathrm{kHz}$ at $400 \mathrm{~W}$ for $0,5,20,40 \mathrm{~min}$ ) on the SEM micrographs of lyophilized CSPI and DGH. (A) CSPI-0 min, (B) CSPI-5 min, (C) CSPI-20 min, (D) CSPI-40 min; (E) DGH-0 min, (F) DGH-5 min, (G) DGH-20 min, (H) DGH-40 min.

\subsubsection{Gelling property}

The formation of soluble aggregates is beneficial to the gelling properties of soy proteins, since they are known as the intermediate product during the heat-induced gelation of soy proteins [106]. Therefore, the thermal dynamic gelation of DGH and CSPI after 20 min of HIU treatment was measured by small amplitude oscillatory test. As shown in Fig. 3.8, both samples showed a typical heat-induced gelation profile, as $\mathrm{G}^{\prime}$ and $\mathrm{G}^{\prime \prime}$ increased after a cycle of heating and cooling (Fig. 3.8). The cross point of $\mathrm{G}^{\prime}$ and $\mathrm{G}^{\prime \prime}$ is usually viewed as the onset of gelation. For CSPI, the onset time was around $1900 \mathrm{~s}$ when the temperature reached 95 ${ }^{\circ} \mathrm{C}$. The increased $\mathrm{G}^{\prime}$ at this temperature was related to the denaturation of glycinin, by which more unfolded proteins interacted with each other, leading to irreversible protein aggregation and network formation [13]. The onset time and temperature for DGH moved to much earlier values, to $760 \mathrm{~s}$ and $42{ }^{\circ} \mathrm{C}$, respectively. This agreed with above results that the glycinin component in DGH had already been denatured after the selective hydrolysis 66 
and ultrasonication. With the existence of soluble aggregates, the initial protein network was formed earlier in the HIU-treated DGH, suggesting its improved gelling ability. Furthermore, the formed gel of the HIU-treated DGH after the temperature sweep showed a significantly higher $\mathrm{G}^{\prime}(\sim 386 \mathrm{~Pa})$ than its CSPI counterpart ( $\left.169 \mathrm{~Pa}\right)$, indicating a stiffer gel structure. This could be due to the higher solubility of DGH after the HIU treatment, which allowed more protein molecules to be incorporated into the gel network.

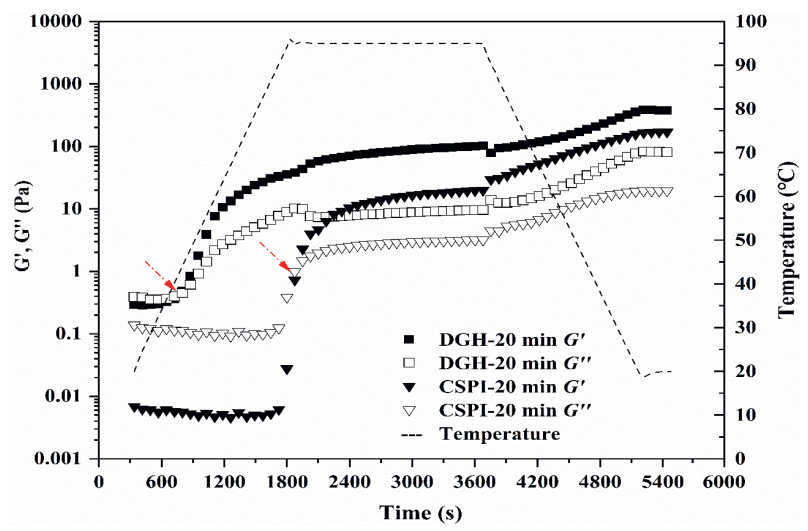

Figure 3.8. The thermal gelation profiles of DGH (rectangle) and CSPI (triangle) after being treated by HIU for $20 \mathrm{~min}$. The solid symbols indicate $\mathrm{G}^{\prime}$, while the open symbols indicate $\mathrm{G}^{\prime \prime}$. The red arrow indicates the onset gelling point.

\subsection{Conclusion}

In this work, selective proteolysis specifically hydrolyzed the glycinin component in SPI, which destroyed its structural integrity and released small protein fractions. As a result, the resultant hydrolysate (DGH) showed different physical, structural, and aggregation properties under the subsequent HIU treatment ( $20 \mathrm{kHz}$ at $400 \mathrm{~W}$ for 0, 5, 20, and $40 \mathrm{~min}$ ), compared with the control SPI (CSPI). HIU induced the formation of soy protein soluble aggregates in the early stage ( $\leq 20 \mathrm{~min}$ ), while it disassociated them after a longer duration (40 min). Compared with CSPI, the DGH nanoparticles formed soluble aggregates with a larger size and higher stability, which could be attributed to the existence of small protein fractions and the larger extent of hydrophobic interactions as well as disulfide bonds. Correspondingly, a higher solubility, more ordered secondary structures, and less polar microenvironment of amino acid residues were observed in $\mathrm{DGH}$, especially at the intermediate time (20 min). After freeze drying, the HIU-treated DGH showed a denser crystalline structure with a smooth surface. The superior solubility and gelling ability shown by the HIU-treated DGH indicated the combination of selective proteolysis, and HIU could be a novel method for soy protein modification. 


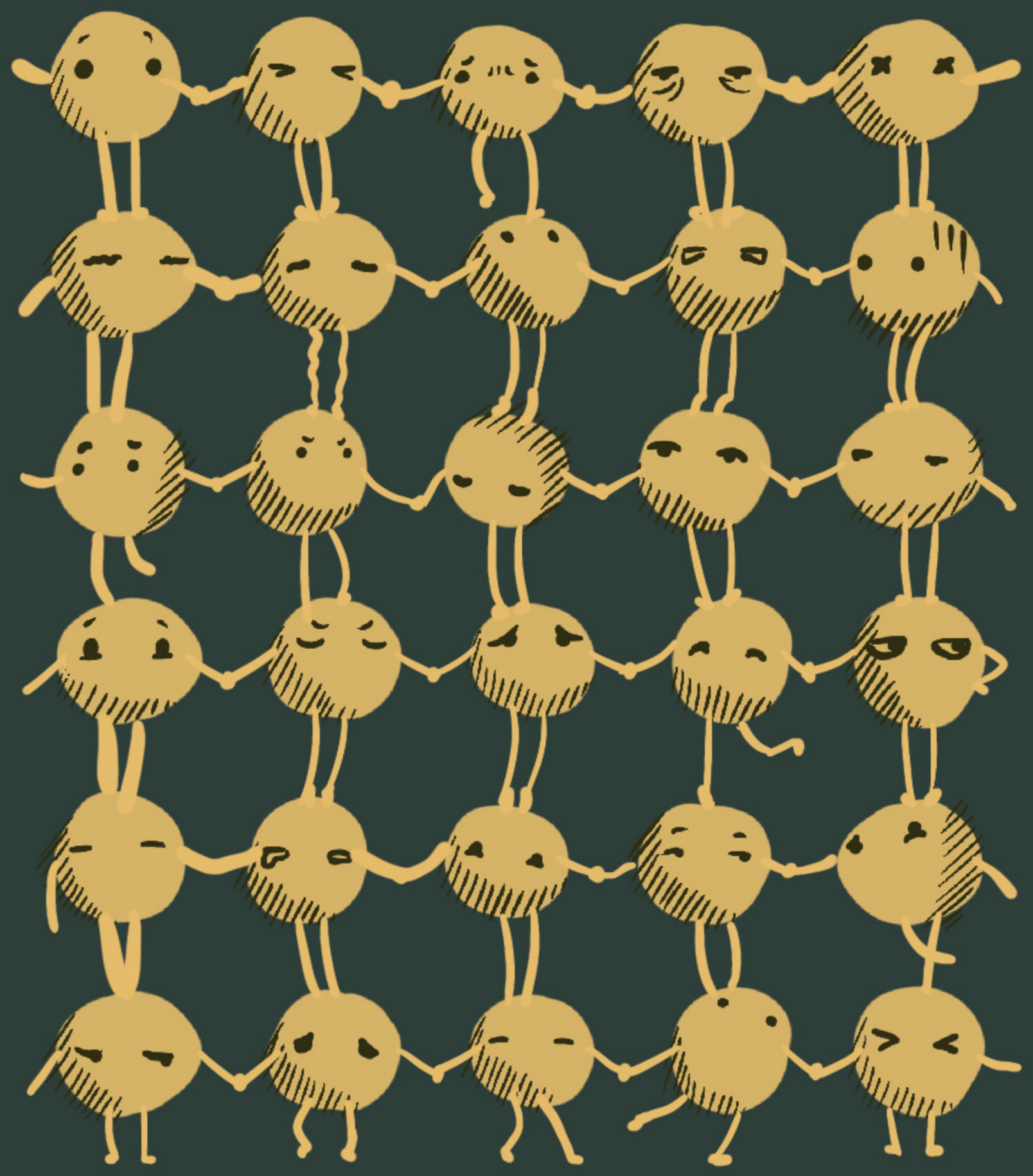




\section{Chapter 4}

\section{Linear and non-linear rheology of heat-set soy protein gels: Effects of selective proteolysis of $\beta$-conglycinin and glycinin}

\section{Published as:}

W. Xia, W.K. Siu, L.M.C. Sagis, Linear and non-linear rheology of heat-set soy protein gels: Effects of selective proteolysis of $\beta$-conglycinin and glycinin, Food Hydrocolloids. 120 (2021) 106962. doi:10.1016/j.foodhyd.2021.106962. 


\section{Abstract}

Soy protein is widely used in the food industry as a gelling agent in many food products. Selectively hydrolyzing one of the two main components ( $\beta$-conglycinin and glycinin) of soy protein is expected to modify its gelling ability in a novel way. To explore these modifications, selective proteolysis was applied on native soy protein isolate (SPI). Degraded $\beta$-conglycinin hydrolysate (DBH) and degraded glycinin hydrolysate (DGH) were obtained with a similar degree of hydrolysis (DH), as verified by SDS-PAGE and pH-stat. Heat-set gels were formed by SPI, hydrolysates, and dialyzed hydrolysates at different protein concentrations (6\%, $10 \%, 14 \%)$. Rheological properties of these gels in the linear regime were determined by small amplitude oscillatory shear (SAOS) tests, while their non-linear rheology was studied by large amplitude oscillatory shear (LAOS) tests. Scaling theory, CLSM, and SEM images were used to link the rheological behavior to differences in the gel microstructure.

Our results showed that hydrolysates had a shorter gelling time and lower gelling temperature, but the non-network peptides made hydrolysate gels less elastic and resulted in a lower critical linear strain than SPI gels. At low concentration and DH, DBH not only had a shorter gelling time but also formed stiffer gels than SPI, which could be due to increased hydrophobic interactions and disulphide bonds, as well as the more homogeneous gel network. On the other hand, DGH formed weaker gels than SPI at every concentration, and their gel structure was coarse, consisting of small and branched flocs. With increased strain amplitude, all gels showed a transition from predominantly elastic to plastic behavior. $\mathrm{D} \beta \mathrm{H}$ displayed intercycle strain softening, while DGH and SPI displayed a weak and strong strain overshoot, respectively. In the medium strain range, SPI showed stronger intracycle shear stiffening and $\mathrm{D} \beta \mathrm{H}$ gels showed stronger intracycle shear thinning. At the large strain regime, SPI and DBH gels showed abrupt yielding behavior while DGH gels yielded more gradually. Overall, the "rheological fingerprint" of soy protein heat-set gels was established, and these rheological properties can be tailored by selectively hydrolyzing different components of soy protein, and varying the $\mathrm{DH}$. 


\subsection{Introduction}

Soy proteins have been extensively used in processed foods because of their high nutritional value, availability, and their contribution to food texture due to their gelling ability [124]. With the increased awareness of the nutritional value of food protein hydrolysates, there is also a need to understand how the gelling properties of soy protein hydrolysates differ from native protein substrates. Gelation of globular proteins is usually induced by heating, during which several reactions can occur, such as protein denaturation/unfolding, dissociation, association, and aggregation, leading to the formation of a 3D network $[125,126]$. The properties of heat-set soy protein gels depend on the thermal gelation conditions (such as temperature, incubation time, $\mathrm{pH}$, and ionic strength), the protein concentration, as well as the protein composition $[10,12,13,71,127,128]$. Like many other plant proteins, soy protein is a multi-component protein mixture with two major components, i.e., $\beta$-conglycinin (7S) and glycinin (11S). These two globulin components not only have different structures and molecular weight (MW) $[33,129]$, but also possess different thermal transition temperatures and gel-forming properties $[13,128,130-132]$. Therefore, understanding how the gelation behavior was affected by hydrolyzing these components is essential for designing new gel products based on soy protein and its hydrolysates.

Gelling properties of hydrolysates from oat protein [133], pea protein [134], and soy protein $[135,136]$ have been studied, and divergent results were reported. Proteolysis breaks the peptide bonds of proteins, and produces molecules with smaller size and lower MW, which might limit their ability to form strong gel networks. This view was supported by the observation that a transglutaminase treatment after hydrolysis restored the gelation properties of hydrolysates to some extent [137]. On the other hand, for native proteins (especially globular proteins), proteolysis cleaves and unfolds their native structure, which is often accompanied by the exposure of hydrophobic and ionizable groups from the interior of the structure. This can contribute to a higher extent of protein-protein interactions and thus favor the gelling ability of hydrolysates $[133,138]$. The changes in functionality caused by proteolysis are highly related to the extent of enzymatic reactions, i.e., the degree of hydrolysis (DH). DH represents the proportion of cleaved peptide bonds, but when the substrate is a multi-component protein mixture like soy protein, it is hard to control which component of the mixture is hydrolyzed. Recently, a method called selective proteolysis has been proposed and studied for soy protein $[31,39,98,100]$. This method makes it possible to specifically hydrolyze one component in soy protein while retaining the others. With this method, the gelation [31], emulsifying properties [98,99] and amyloid fibrillation [100] of SPI were modified in a novel way. 
The rheology of food protein gels is important for their practical application. Dynamic oscillatory shear tests are commonly performed, in which a material is subjected to a sinusoidal deformation, and the resulting mechanical response is measured [139]. Based on the amplitude of deformation, dynamic oscillatory shear tests are divided into two categories, i.e., small amplitude oscillatory shear (SAOS) and large amplitude oscillatory shear (LAOS) tests. SAOS tests subject protein gels to sufficiently small strains that will not disrupt the gel microstructure, and characterize the rheological properties in the linear viscoelastic (LVE) regime where the storage modulus ( $\mathbf{G}^{\prime}$ ) and loss modulus (G") both are independent of the magnitude of the applied strain, or strain rate [140]. In LAOS tests, the strain amplitude is increased to a level where the gel microstructure is affected, and the mechanical response of gels falls in the non-linear viscoelastic (NLVE) regime where the viscoelastic moduli ( $G^{\prime}$ and $G^{\prime \prime}$ ) become a function of the strain and strain rate. In food production (e.g., mixing and transportation) and physiological digestion processes (e.g., chewing and swallowing), the stresses and strains (rates) that are applied on food materials can be remarkably high. In spite of this, most studies on food biopolymer gels apply only SAOS tests to investigate their LVE behavior, and only a few studies have reported data in the NLVE regime.

Melito et al. measured NLVE properties of agarose gels, whey protein isolate/ $k$-carrageenan gels and commercial cheese via LAOS tests. Their results suggested that the sensory and oral processing characteristics of food gels can be well correlated to their NLVE properties [140-143]. Bi et al. found that acid-induced hybrid gels of SPI and locust bean $\mathrm{gum} / \mathrm{K}$ Carrageenan displayed strain thinning behavior in the NLVE regime [144,145]. By the Fourier transformation of the LAOS data, they validated a new definition of the critical point in acid-induced pea protein gels, showing the applicability of non-linear rheology in calculating the fractal dimension $\left(\boldsymbol{D}_{\boldsymbol{f}}\right)$ of isotropic food gels [146]. Precha-Atsawanan et al. compared the rheological behavior of native (WX) and debranched (DB) waxy rice starch gels in both LVE and NLVE regimes, which showed that WX starch gels had a wider LVE range and higher loss tangent (tan $\mathbf{\delta}$ ) than DB starch gel, while DB starch gels showed more abrupt yielding and stronger softening behavior in NLVE regime [147]. More recently, comprehensive studies using both SAOS and LAOS tests on heat-set pea protein gels [148], fermentation-induced pea protein gels $[134,149]$, and various concentrated protein systems (Pea and soy protein and wheat gluten) [150] have been documented. The insights into non-linear rheology enabled these studies to provide a clearer and more detailed guide for designing novel plant protein gel-based foods like pea protein yoghurt and meat analogues.

The rheological properties of soy protein heat-set gels in both LVE and NLVE regimes (the "rheological fingerprint"), and how these rheological properties are affected by selective proteolysis of soy protein has not been systematically investigated yet. Therefore, in this 
study, we selectively hydrolyzed the $\beta$-conglycinin or glycinin component of native soy protein isolate (SPI). Two types of soy protein hydrolysates, referred to as degraded $\beta$ conglycinin hydrolysate (DßH) and degraded glycinin hydrolysate (DGH) were obtained, with a similar DH. After that, heat-set gels from SPI, hydrolysates, and dialyzed hydrolysates were prepared at various protein concentrations. The linear and non-linear rheology of these gels were studied by SAOS and LAOS tests. Finally, scaling theory, confocal laser scanning microscopy (CLSM) and scanning electron microscopy (SEM) were used to correlate the rheological properties of these gels with their microstructures. We will show that the rheological fingerprint of heat-set soy protein gels can be tailored by selective proteolysis.

\subsection{Materials and methods}

\subsubsection{Materials}

Defatted and lightly toasted soy flour (SOPRO-UTB 100, dry-based protein content 50\%) was provided by Barentz International B.V. (Hoofddorp, the Netherlands). Papain (article No. P4762, 13 units/mg), pepsin (article No. P6887, 3412 units/mg), and antipain (article No.10791, >50000 U/mg) were purchased from Sigma-Aldrich, Inc. (St Louis, MO, USA). Other reagents used were of analytical grade or biochemical grade. Deionized distilled water (DDW) used in the study was purified by PURELAB Ultra apparatus from ELGA LabWater Co. (High Wycombe, UK). Unless mentioned otherwise, all the experiments were performed at room temperature $\left(20 \pm 1^{\circ} \mathrm{C}\right)$.

\subsubsection{Preparation of native soy protein isolate (SPI)}

SPI with its protein in a native state was extracted from defatted soy flour according to the method of Wagner et al. [45]. Defatted soy flour was dissolved in DDW (flour/water ratio was $1: 10 \mathrm{w} / \mathrm{w}$ ) and the resultant solution was adjusted to $\mathrm{pH} 7.5$ and stirred for $2 \mathrm{~h}$. After that, the soy flour solution was centrifuged at $5000 \mathrm{~g}$ for 30 min (Avanti J-26 XP centrifuge, Beckman Coulter, Inc., Brea, CA, USA) and the supernatant was collected. The supernatant was adjusted to $\mathrm{pH} 4.5$ with $2 \mathrm{~mol} / \mathrm{L} \mathrm{HCl}$ and then centrifuged at $5000 \mathrm{~g}$ for $10 \mathrm{~min}$ to obtain a precipitate. The precipitate was redissolved in DDW $(1: 4 \mathrm{w} / \mathrm{w})$ and adjusted to $\mathrm{pH} 7.0$ with $2 \mathrm{~mol} / \mathrm{L} \mathrm{NaOH}$. Finally, the solution was lyophilized by a freeze dryer (Martin Christ Gefriertrocknungsanlagen $\mathrm{GmbH}$, Osterode am Harz, Germany), and the obtained SPI powder was kept in an airtight container, stored at $4{ }^{\circ} \mathrm{C}$. The protein content of SPI was 96 $\pm 1 \%$ (dry weight, $N \times 6.25$ ), determined by Dumas combustion method with a FlashEA 1112 N/Protein Analyzer (Thermo Fisher Scientific, Waltham, MA, USA). 


\subsubsection{The reaction kinetics of selective proteolysis}

The reaction kinetics of selective proteolysis were investigated by a pH-stat device according to the method of Mat et al. [30]. In brief, protein proteolysis at different pH conditions leads to the release or consumption of protons. pH-stat devices automatically add acidic or basic titrant to keep the $\mathrm{pH}$ constant during proteolysis. The enzymatic reaction rate can be inferred from the titration rate, and the $\mathrm{DH}$ can be calculated from the quantity of titrant added. SPI dispersions (5g SPI powder in $100 \mathrm{~mL} \mathrm{DDW}$ ) were prepared and transferred to jacketed beakers. The $\mathrm{pH}$ of SPI dispersions was monitored by an automatic $\mathrm{pH}$-stat titration device (Metrohm, Herisau, Switzerland). Papain and pepsin were used to selectively hydrolyze $\beta$-conglycinin and glycinin, respectively. Two enzyme to SPI ratios (E/S ratio) were adopted: E/S $0.02 \%$ and E/S 0.05\%, similar to previous studies $[39,98,100]$.

The reaction conditions of selective proteolysis of $\beta$-conglycinin were $75^{\circ} \mathrm{C}$ and $\mathrm{pH} 7.0$. Basic titrant $(1 \mathrm{~mol} / \mathrm{L} \mathrm{NaOH})$ was used to maintain the $\mathrm{pH}$ at 7.0. The $\mathrm{DH}$ was calculated using equation (1):

$$
D H(\%)=\frac{V_{\text {base }} \times N_{\text {base }}}{m \times h_{\text {tot }}} \times \frac{1}{\alpha_{\mathrm{NH}_{2}}} \times 100
$$

where $V_{\text {base }}$ and $N_{\text {base }}$ are the volume $(\mathrm{mL})$ and normality $(\mathrm{mol} / \mathrm{L})$ of basic titrant respectively, $m$ is the protein mass $(\mathrm{g})$, and $h_{\text {tot }}$ is the number of peptide bonds per gram of proteins (for soy protein $h_{t o t}=7.8 \mathrm{~mol} / \mathrm{kg}$ protein) $; \alpha_{\mathrm{NH}_{2}}$ is the mean degree of dissociation of amino groups [151].

The reaction conditions of selective proteolysis of glycinin were $37^{\circ} \mathrm{C}$ and $\mathrm{pH} 2.0$. Acidic titrant $(1 \mathrm{~mol} / \mathrm{L} \mathrm{HCl})$ was used to maintain the $\mathrm{pH}$ at 2.0. The $\mathrm{DH}$ was calculated from equation (2):

$$
D H(\%)=\frac{V_{a c i d} \times N_{\text {acid }}}{m \times h_{\text {tot }}} \times \frac{1}{1-\alpha_{C O O H}} \times 100
$$

where $V_{\text {acid }}$ and $N_{\text {acid }}$ are the volume $(\mathrm{mL})$ and normality $(\mathrm{mol} / \mathrm{L})$ of acidic titrant respectively, $\alpha_{\mathrm{COOH}}$ is the mean degree of dissociation of the carboxyl groups [151].

\subsubsection{Preparation of soy protein hydrolysates}

Four batches of SPI dispersions (25g SPI powder in $500 \mathrm{~mL}$ DDW) were prepared in advance. Papain and pepsin were dissolved in DDW at a concentration of $1 \mathrm{mg} / \mathrm{mL}$ before use. For the preparation of degraded $\beta$-conglycinin hydrolysate $(\mathbf{D} \boldsymbol{\beta} \mathbf{H})$, two batches of SPI dispersions were maintained at $\mathrm{pH} 7.0$ and $75^{\circ} \mathrm{C}$. We added $5 \mathrm{~mL}$ of papain solution to one batch (E/S 
$0.02 \%$ ), followed by incubation for $30 \mathrm{~min}$, while to the other batch $12.5 \mathrm{~mL}$ of papain solution was added (E/S $0.05 \%$ ), after which it was incubated for $90 \mathrm{~min}$. The reaction was terminated by adding a 10 -fold molar amount of antipain. The resultant hydrolysates were $\mathrm{D} \beta \mathrm{H}$ with a lower $\mathrm{DH}$ and a higher $\mathrm{DH}$, respectively. For the preparation of degraded glycinin hydrolysate (DGH), two batches of SPI dispersion were maintained at pH 2.0 and $37^{\circ} \mathrm{C}$. To one batch $5 \mathrm{~mL}$ of pepsin solution was added (E/S $0.02 \%$ ), followed by incubation for 40 $\mathrm{min}$, while to the other batch $12.5 \mathrm{~mL}$ of pepsin solution was added (E/S $0.05 \%$ ), after which it was incubated for $50 \mathrm{~min}$. The reaction was terminated by neutralizing to $\mathrm{pH} 7.0$ and boiling for $5 \mathrm{~min}$. The resultant hydrolysates were DGH with a lower $\mathrm{DH}$ and a higher $\mathrm{DH}$, respectively.

To reveal the effects of small peptides on the gel properties of different hydrolysates, each hydrolysate mentioned above was further divided into two portions. One portion was lyophilized directly while another portion was put into regenerated cellulose membrane tubing (MWCO: 12-14 kDa; Spectra/Por ${ }^{\circledR} 4$, Repligen, Waltham, MA, USA) and dialyzed against DDW at $4{ }^{\circ} \mathrm{C}$ for two days before lyophilization. Therefore, eight hydrolysate samples in total were investigated in this study, and the abbreviation of them is composed of the type, DH level, and whether dialyzed or not. A graphic illustration of the preparation and abbreviation of these hydrolysate samples is shown in Fig. 4.1 .

Figure 4.1. Graphic illustration of the sample preparation (A) and abbreviation list (B).

(A)

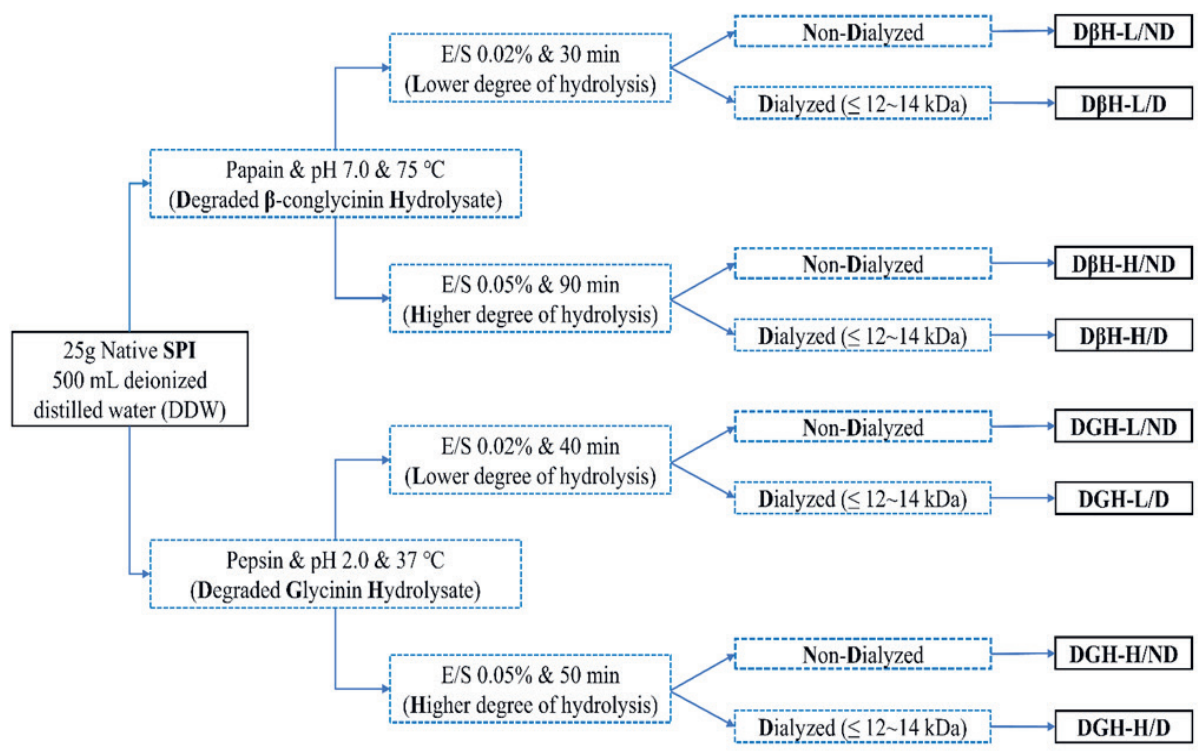


Figure 4.1. (B)

\begin{tabular}{|c|c|}
\hline MW: molecular weight & $\begin{array}{l}\mathbf{S}_{\Delta \mathbf{G}^{\prime}} \text { : the average slope of } \mathrm{G}^{\prime} \text { in } 95{ }^{\circ} \mathrm{C} \\
\text { heating }\end{array}$ \\
\hline DH: degree of hydrolysis & $\boldsymbol{D}_{\boldsymbol{f}}$ : fractal dimension \\
\hline E/S: enzyme to SPI ratio & $\boldsymbol{V c}$ : critical linear strain \\
\hline SAOS: small amplitude oscillatory shear & $\boldsymbol{\sigma}:$ shear stress \\
\hline LAOS: large amplitude oscillatory shear & $\boldsymbol{Y o}_{\mathrm{o}}$ : intercycle strain \\
\hline LVE: linear viscoelastic & $\dot{\gamma}_{0}:$ intercycle strain rate \\
\hline NLVE: non-linear viscoelastic & $\mathbf{y}$ : intracycle strain \\
\hline $\mathbf{G}^{\prime}$ : storage modulus & $\dot{\mathbf{v}}$ : intracycle strain rate \\
\hline G": loss modulus & $\boldsymbol{\sigma}_{\mathbf{e}}:$ decomposed elastic stress \\
\hline $\tan \boldsymbol{\delta}:$ loss tangent & $\boldsymbol{\sigma}_{\mathbf{v}}:$ decomposed viscous stress \\
\hline $\mathbf{t}_{\text {gel }}$ : the onset time of gelation & CLSM: confocal laser scanning microscopy \\
\hline $\mathbf{T}_{\mathbf{g e l}}$ : the onset temperature of gelation & SEM: scanning electron microscopy \\
\hline
\end{tabular}

\subsubsection{Sodium dodecyl sulfate-polyacrylamide gel electrophoresis (SDS-PAGE)}

The protein dispersion $(2 \mathrm{mg} / \mathrm{mL}$ ) was mixed with a $2 \times$ Laemmli sample buffer (Bio-Rad Laboratories Inc., Hercules, CA, USA) at a ratio of $1: 1$, and heated at $95{ }^{\circ} \mathrm{C}$ for $5 \mathrm{~min}$ in a thermomixer (Eppendorf AG, Hamburg, Germany). Afterward, $10 \mu \mathrm{L}$ molecular weight markers (Precision Plus Protein ${ }^{\mathrm{TM}}$, Bio-Rad) and $20 \mu \mathrm{L}$ of each sample solution were loaded on a $12 \%$ Tris- $\mathrm{HCl}$ Mini-PROTEAN TGX ${ }^{\mathrm{TM}}$ Precast Gel (Bio-Rad). A premixed electrophoresis running buffer (10x Tris/glycine/SDS \# 1610772, Bio-Rad) was used, and the electrophoresis 
was carried out by a Mini-PROTEIN Tetra cell (Bio-Rad) at $200 \mathrm{~V}$ for about $30 \mathrm{~min}$. After that, the gels were stained with Coomassie Brilliant Blue R-250 (Bio-Rad) for 1 h followed by destaining with DDW. Finally, the gel was scanned and analyzed by a GS-900 Calibrated Densitometer with Image Lab software (Bio-Rad).

\subsubsection{Rheological measurements}

The heat-induced gelation process of different samples and the rheological properties of the resulting gels were studied with an MCR 302 rheometer (Anton Paar, Graz, Austria) and titanium sandblasted concentric cylinder geometry (CC17). Lyophilized samples were mixed with DDW to three protein concentrations $(6 \%, 10 \%$, and $14 \% \mathrm{w} / \mathrm{w})$ and stirred overnight to ensure full hydration. The $\mathrm{pH}$ of sample dispersions was adjusted to 7.0 by adding $2 \mathrm{~mol} / \mathrm{L}$ $\mathrm{NaOH}$ or $\mathrm{HCl}$. The conductivity of dispersions was standardized to $25 \mathrm{mS} / \mathrm{cm}$ by adding $\mathrm{NaCl}$, which was equivalent to the conductivity of a $250 \mathrm{mM} \mathrm{NaCl}$ salt concentration. The dispersions were then degassed with a vacuum pump. A volume of $4.7 \mathrm{ml}$ protein dispersion was slowly injected into the cup of the CC17 geometry to avoid bubble formation. During the rheological measurements, the temperature was controlled by a Peltier element and a water circulation system, and the cup was covered with a solvent trap for preventing sample evaporation.

\section{Small amplitude oscillatory shear (SAOS) tests}

SAOS tests were performed at a fixed amplitude $(1 \%)$ and frequency $(1 \mathrm{~Hz})$, which were within the LVE regime (verified by intercycle strain sweep tests). The samples were first allowed to rest at $20^{\circ} \mathrm{C}$ for 5 min before being subjected to a temperature ramp from $20^{\circ} \mathrm{C}$ to $95{ }^{\circ} \mathrm{C}$ at $3{ }^{\circ} \mathrm{C} / \mathrm{min}$. After that, the temperature was maintained at $95{ }^{\circ} \mathrm{C}$ for $30 \mathrm{~min}$ and then cooled back to $20^{\circ} \mathrm{C}$ at the same rate $\left(3^{\circ} \mathrm{C} / \mathrm{min}\right)$. Finally, the resultant soy protein gels were kept at $20^{\circ} \mathrm{C}$ for $5 \mathrm{~min}$ in the rheometer before further tests were performed. The $\mathrm{G}^{\prime}, \mathrm{G}^{\prime \prime}$, and $\tan \delta$ were recorded as a function of time throughout the experiment.

\section{Large amplitude oscillatory shear (LAOS) tests}

LAOS amplitude-sweep tests were performed to investigate the viscoelastic behavior of soy protein gels in the NLVE regime. The heat-set gels were subjected to a strain amplitude sweep from $1 \%$ to $1000 \%$ (logarithmic ramp mode), at a constant frequency of $1 \mathrm{~Hz}$ and 20 ${ }^{\circ} \mathrm{C}$. The $\mathrm{G}^{\prime}$ and $\mathrm{G}$ " were recorded as a function of intercycle strain $(\boldsymbol{Y o})$, while shear stress $(\boldsymbol{\sigma})$ was recorded as a function of intracycle strain $(\boldsymbol{\gamma})$ and strain rate $(\dot{\gamma})$. Lissajous plots (also called Lissajous-Bowditch plots) were constructed to analyze the non-linear rheological response of these gels. This analysis framework was extensively discussed by Ewoldt et al. [25]. The different NLVE properties of gels can be directly reflected by the differences in 
their Lissajous plots. The intracycle strain stiffening and intracycle shear thickening behaviors can be quantified by a S-factor and a T-factor, respectively, as defined by equation (3) and (4):

$$
\begin{gathered}
S=\frac{G_{L}^{\prime}-G_{M}^{\prime}}{G_{L}^{\prime}} \\
T=\frac{\eta_{L}^{\prime}-\eta_{M}^{\prime}}{\eta_{L}^{\prime}}
\end{gathered}
$$

In these equations, $G_{L}^{\prime}$ is the large-strain elastic modulus, also referred to as the secant modulus, $G_{M}^{\prime}$ is the shear elastic modulus at the minimum strain, also referred to as the tangent modulus at strain zero; $\boldsymbol{\eta}_{L}^{\prime}$ is the viscosity at the maximum shear rate, and $\boldsymbol{\eta}_{M}^{\prime}$ is the viscosity at the minimum shear rate [25]. Based on these parameters, $S=0$ indicates linear elastic behavior, $\mathrm{S}>0$ indicates intracycle strain stiffening, and $\mathrm{S}<0$ indicates intracycle strain softening; $\mathrm{T}=0$ indicates linear viscous behavior, $\mathrm{T}>0$ indicates intracycle shear thickening, and $\mathrm{T}<0$ indicates intracycle shear thinning. The plots of $G_{L}^{\prime}, G_{M}^{\prime}, \eta_{L}^{\prime}$, and $\eta_{M}^{\prime}$ vs. Yo can be found in supplementary information (SI).

\subsubsection{Confocal laser scanning microscopy (CLSM)}

Protein sample dispersions $(10 \% \mathrm{w} / \mathrm{w}$ ) prepared in Section 4.2.6 were stained by $0.05 \%$ $\mathrm{w} / \mathrm{w}$ Rhodamine B. Pre-stained dispersions $(60 \mu \mathrm{L})$ were added to hermetically sealed flat cuvettes (Gene Frame ${ }^{\circledR} 125 \mu \mathrm{L}$, Thermo Fisher Scientific, Waltham, MA, USA) which were glued on microscopy slides in advance, and then covered with coverslips. Gels were prepared by heating these cuvettes in a water bath (JULABO GmbH, Seelbach, Germany), equivalent to the process in the SAOS tests. After heating, the cuvettes were cooled in DDW to $20^{\circ} \mathrm{C}$. CLSM imaging of the gels was performed at room temperature with a Zeiss LSM 510 META confocal microscope equipped with an Axiovert 200M inverted microscope. A He/Ne $543 \mathrm{~nm}$ laser was used to excite the Rhodamine B $(570 \sim 590 \mathrm{~nm})$. Images $(512 \times 512$ pixels $)$ were captured at a sample depth of $5 \mu \mathrm{m}$ behind the coverslip.

\subsubsection{Scanning electron microscopy (SEM)}

Aliquots $(3 \mu \mathrm{m})$ of sample dispersions $(10 \% \mathrm{w} / \mathrm{w})$ prepared in Section 4.2.6 were transferred into a disposable thermotolerant syringe (Terumo Corporation, Tokyo, Japan) with the tip sealed by a syringe screw cap (Combi ${ }^{\text {Tm }}$ plug, Becton Dickinson, Franklin Lakes, $\mathrm{NJ}$, USA). The syringes were then put vertically in a rack and heated in a water bath as described in Section 4.2.7. The top of the syringe was also sealed by aluminum foil to prevent sample evaporation during heating. After heating, the syringe was cooled in DDW to $20^{\circ} \mathrm{C}$. For SEM imaging, the gels were cut into small cubes $(\sim 5 \times 5 \times 5 \mathrm{~mm})$ and fixed in 
$2.5 \%$ glutaraldehyde for at least 8 hours. After the crosslinking, the samples were rinsed to remove the excess of glutaraldehyde and were dehydrated using a series of ethanol $(30 \%$ to $100 \%$ ) solutions followed by critical point drying (CPD 300, Leica, Vienna, Austria). Subsequently, the samples were fractured and attached to SEM sample holders using Carbon Conductive Cement (Leit-C, Neubauer Chemicalien, Germany). To remove all the solvent from the adhesive, the samples were stored overnight under a vacuum. After sputter coating with a $12 \mathrm{~nm}$ layer of tungsten (SCD 500, Leica, Vienna, Austria), the fractured surfaces were analyzed with SE detection at $2 \mathrm{kV}$ in a field emission scanning electron microscope (Magellan 400, FEI, Eindhoven, the Netherlands).

\subsubsection{Statistical analysis}

All measurements were performed in triplicate unless otherwise stated, and the results were presented as means \pm standard deviation. The statistical analysis on sample replicates was conducted by SPSS 25.0 (IBM SPSS Inc. Chicago, IL, USA). One-way ANOVA (one-way analysis of variance) with Duncan's method $(p<0.05)$ were used to evaluate the statistical significance of differences among means. Lissajous plots were made by MATLAB R2018b (MathWorks Inc., MA, USA) while other figures were made by Origin 2018 (Origin Lab Corporation, MA, USA).

\subsection{Results and discussion}

\subsubsection{Selective proteolysis of soy protein}

\section{Degree of hydrolysis (DH) for selective proteolysis of different components}

The $\mathrm{DH}$ of selective proteolysis was studied by the $\mathrm{pH}$-stat method and a plot of $\mathrm{DH}$ vs. incubation time is shown in Fig. 4.2. In both the selective proteolysis of $\beta$-conglycinin and glycinin, the reaction rate was faster at the beginning and then slowed down with incubation time. This exponential feature is typical for enzymatic reactions [136,152,153]. Increasing the $\mathrm{E} / \mathrm{S}$ ratio from $0.02 \%$ to $0.05 \%$ resulted in a higher $\mathrm{DH}$ at the same incubation time, but this increase in $\mathrm{DH}$ was more significant in the selective proteolysis of glycinin, which could be due to the higher content of glycinin in SPI as shown later.

Based on Fig. 4.2, incubation times of $30 \mathrm{~min}$ and $90 \mathrm{~min}$ were chosen when selectively hydrolyzing $\beta$-conglycinin at $E / S$ ratio of $0.02 \%$ and $0.05 \%$, to prepare degraded $\beta$ conglycinin hydrolysate $(\mathbf{D} \boldsymbol{\beta H})$ with a lower $\mathrm{DH}(1.55 \pm 0.01 \%)$ and a higher $\mathrm{DH}$ $(2.46 \pm 0.15 \%)$, respectively. An incubation time of $40 \mathrm{~min}$ or $50 \mathrm{~min}$ was chosen when selectively hydrolyzing glycinin at E/S ratio of $0.02 \%$ and $0.05 \%$, to prepare degraded

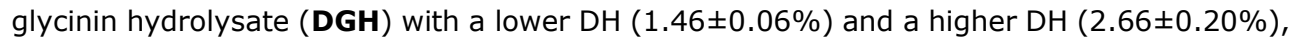


respectively. This way, two different types of hydrolysates could be compared at two similar DH levels.

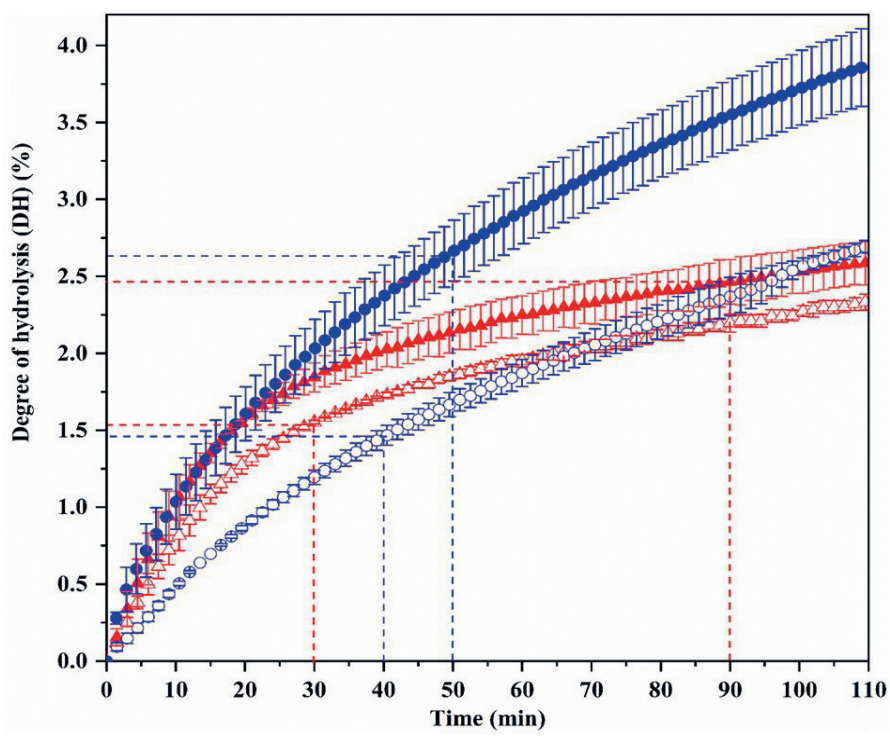

Figure 4.2. The DH as a function of time for selective proteolysis. Red triangles represent selective proteolysis of $\beta$-conglycinin. Blue circles represent selective proteolysis of glycinin. Solid symbols represent the E/S ratio of $0.05 \%$. Open symbols represent the E/S ratio of $0.02 \%$. Dashed lines are guides to the eye.

\section{SDS-PAGE of different hydrolysates}

The SDS-PAGE patterns of native SPI and its hydrolysates after selective proteolysis are shown in Fig. 4.3. Native SPI showed all bands of its two globulin components (lane 2). $\beta$ conglycinin (7S) is a trimeric glycoprotein of three kinds of subunits, $a^{\prime}, a$, and $\beta$, which correspond to the bands at around $80 \mathrm{kDa}, 70 \mathrm{kDa}$ and $50 \mathrm{kDa}$, respectively. Glycinin (11S) is a hexamer protein, and each subunit consists of acidic (A) and a basic (B) polypeptide linked by disulfide bonds. The bands at around $40 \mathrm{kDa}, 35 \mathrm{kDa}$, and $20 \mathrm{kDa}$ are assigned to the $A_{3}, A_{1,2,4}$, and $B$ polypeptides, respectively. The faint band at $95 \mathrm{kDa}$ was most likely the lipoxygenase, and it vanished gradually after the selective proteolysis of $\beta$-conglycinin (lane 3 \& lane 4), which was also found by Tsumura et al. [31]. 
Figure 4.3. SDS-PAGE patterns for native SPI and its hydrolysates after selective proteolysis. Lane 1: Marker;

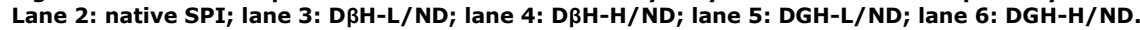

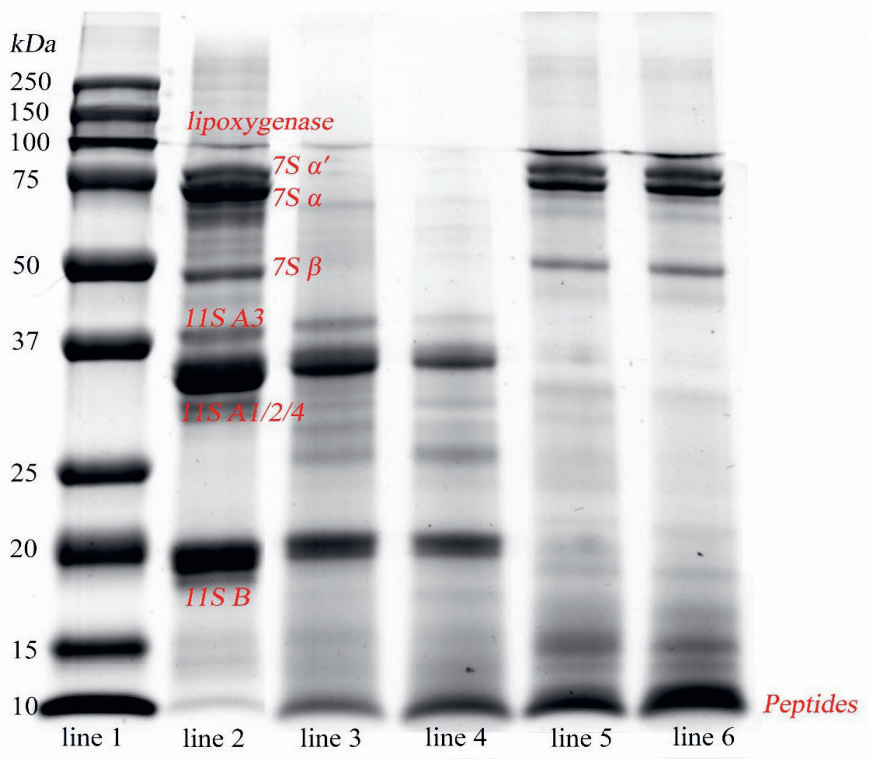

The bands of $a^{\prime}, a$, and $\beta$ subunits of $\beta$-conglycinin all became faint in D $B H-L / N D$ (lane 3) and almost completely disappeared in $\mathrm{D} \beta \mathrm{H}-\mathrm{H} / \mathrm{ND}$ (lane 4), while the bands of glycinin remained visible. On the other hand, the bands corresponding to the acidic and basic polypeptides of glycinin all faded out in DGH-L/ND (lane 5) and DGH-H/ND (lane 6), while the bands of $\beta$-conglycinin subunits barely changed. Table 4.1 shows the relative composition of each sample according to the densitometric analysis of SDS-PAGE patterns. The native SPI extracted in our study contained $29.8 \% \beta$-conglycinin and $61.9 \%$ glycinin, and this ratio of $\beta$-conglycinin to glycinin is in agreement with previous studies $[11,154]$. After selective proteolysis, $\mathrm{D} \beta \mathrm{H}-\mathrm{L} / \mathrm{ND}$ and $\mathrm{D} \beta \mathrm{H}-\mathrm{H} / \mathrm{ND}$ comprised only $3.3 \%$ and $0.3 \% \beta-$ conglycinin, while DGH-L/ND and DGH-H/ND contained only $4.2 \%$ and $0.8 \%$ glycinin. The content of peptides with MW around $10 \mathrm{kDa}$ increased significantly in each hydrolysate. DGH-L/ND and DGH-H/ND contained about double the amount of these small peptides ( $\sim 10$ $\mathrm{kDa}$ ) compared to their $\mathrm{D} \beta \mathrm{H}$ counterpart, which could be due to the different cleavage mechanisms of pepsin and papain as well as the different structures of glycinin and $\beta$ conglycinin. 
Table 4.1. Relative composition of native SPI and its hydrolysates after selective proteolysis, based on the densitometry analysis of SDS-PAGE patterns.

\begin{tabular}{llllll}
\hline Band & Native SPI & D $3 H-L / N D$ & D $\beta H-H / N D$ & DGH-L/ND & DGH-H/ND \\
\hline Lipoxygenase & $3.9 \%$ & $2.0 \%$ & $0.5 \%$ & $9.4 \%$ & $6.8 \%$ \\
$7 \mathrm{~S} \mathrm{a} \mathrm{a}^{\prime}$ & $4.6 \%$ & $0.3 \%$ & $0.1 \%$ & $7.7 \%$ & $6.1 \%$ \\
$7 \mathrm{~S} \mathrm{a}$ & $13.3 \%$ & $2.5 \%$ & $0.1 \%$ & $12.0 \%$ & $12.1 \%$ \\
$7 \mathrm{~S} \beta$ & $11.9 \%$ & $0.5 \%$ & $0.1 \%$ & $11.7 \%$ & $12.2 \%$ \\
$11 \mathrm{~S} \mathrm{A3}$ & $7.6 \%$ & $5.3 \%$ & $1.7 \%$ & $0.0 \%$ & $0.0 \%$ \\
$11 \mathrm{~S} \mathrm{~A} 1,2,4$ & $46.5 \%$ & $29.5 \%$ & $27.4 \%$ & $3.3 \%$ & $0.2 \%$ \\
$11 \mathrm{~S} \mathrm{~B}$ & $7.8 \%$ & $37.3 \%$ & $35.6 \%$ & $0.9 \%$ & $0.6 \%$ \\
$7 \mathrm{~S}$ total & $29.8 \%$ & $3.3 \%$ & $0.3 \%$ & $31.4 \%$ & $30.4 \%$ \\
$11 \mathrm{~S}$ total & $61.9 \%$ & $72.1 \%$ & $64.7 \%$ & $4.2 \%$ & $0.8 \%$ \\
Peptides & $4.4 \%$ & $22.6 \%$ & $34.5 \%$ & $55.1 \%$ & $62.0 \%$ \\
$(\sim 10 \mathrm{kDa})$ & & & & &
\end{tabular}

\subsubsection{Linear rheology}

\section{Heat-induced gelation profile}

SAOS tests were applied to non-destructively characterize the heat-induced gelation process of different samples and the linear viscoelastic properties of formed gels. Fig. 4.4A presents the typical gelation profiles of native SPI, showing the evolution of $\mathrm{G}^{\prime}$ and $\mathrm{G}^{\prime \prime}$ during the temperature ramp phase, constant $\mathrm{T}\left(95^{\circ} \mathrm{C}\right)$ phase, and cool down. At a certain temperature, $G^{\prime}$ and $G^{\prime \prime}$ started to increase due to protein aggregation and the formation of the initial gel network, which could be driven by non-covalent interactions and disulfide bonds [13]. After this, $G^{\prime}$ and $G^{\prime \prime}$ kept increasing upon heating since more protein was incorporated in the gel network, and rearrangements of the network happened [13]. Finally, a substantial increase in $\mathrm{G}^{\prime}$ and $\mathrm{G}^{\prime \prime}$ was observed in the cooling stage. This gel stiffening during cooling has been found to be almost thermo-reversible, which indicated that it was mostly a result of enhanced intra-/intermolecular hydrogen bond formation and van der Waals interactions $[13,14]$. The thermal gelation curves of D $\beta H-L / N D$ (Fig. S4.1) and D $\beta H-$ $\mathrm{H} / \mathrm{ND}$ (Fig. 4.4B) were similar to those of SPI, while the gelation profiles of DGH-L/ND (Fig. S4.1) and DGH-H/ND (Fig. 4.4C) were significantly different. Instead of a steep increase in the temperature ramp period, the $\mathrm{G}^{\prime}$ and $\mathrm{G}^{\prime \prime}$ of $\mathrm{DGH}$ increased more slowly. The thermal gelation profiles of these hydrolysates did not change significantly after dialysis (see Fig. S4.1). 
Figure 4.4. Heat-induced gelation profiles of different samples (A-C) and strain amplitude sweep tests (D-F) of their heat-set gels. From top to bottom: native SPI, D BH-H/ND, DGH-H/ND. The temperature profile is indicated with a dashed line. The results for the hydrolysates with low DH and the hydrolysates after dialysis can be found in supplementary materials (Fig. S4.1 and Fig. S4.2).
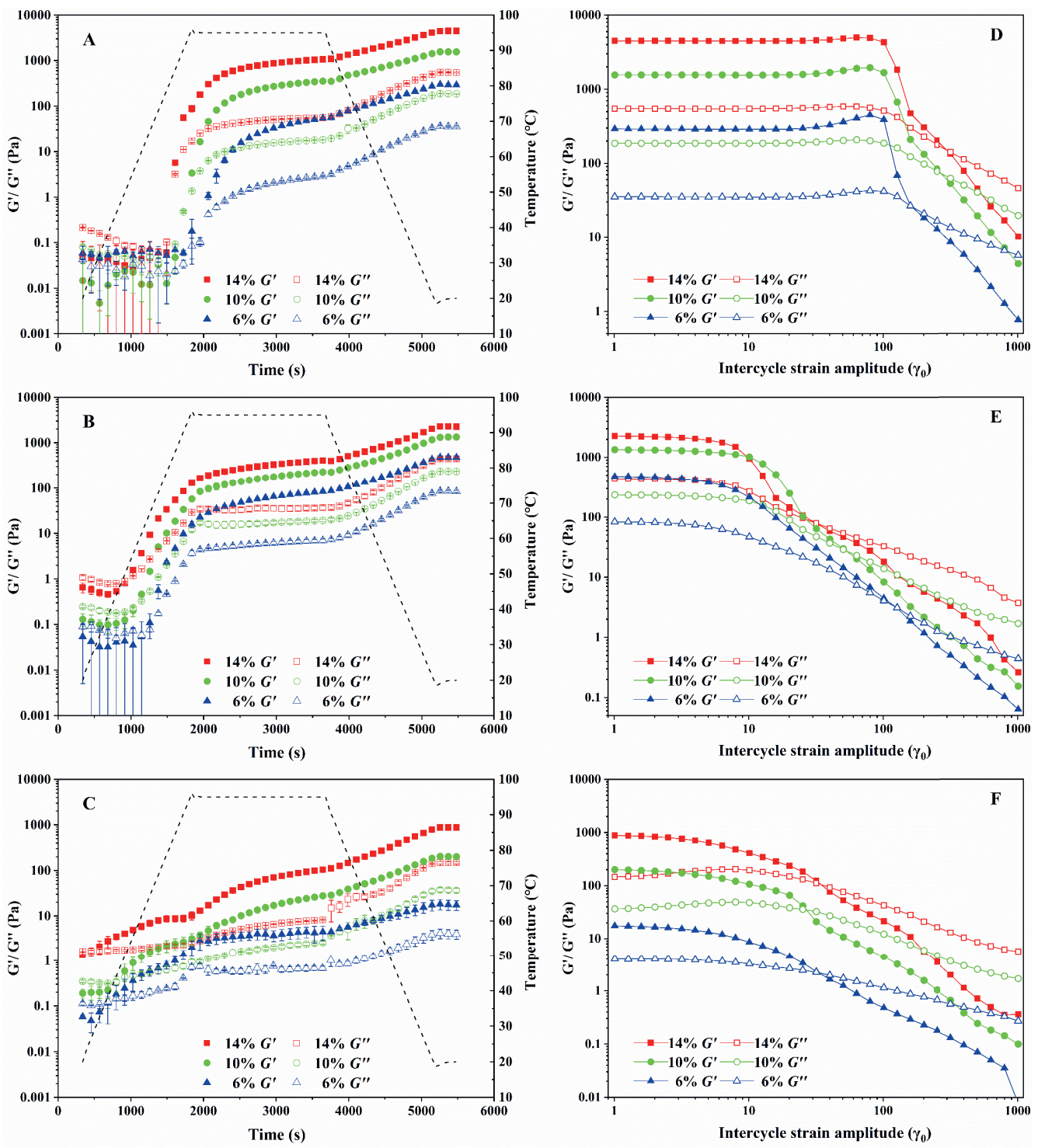

The differences in the initial network formation were further characterized by determining the time ( $\left.\mathbf{t}_{\mathbf{g e l}}\right)$ and temperature ( $\left.\mathbf{T}_{\mathbf{g e l}}\right)$ of the onset of gelation, i.e., the gelling point where $\mathrm{G}^{\prime}=\mathrm{G}^{\prime \prime}$. As shown in Table 4.2, at a fixed temperature ramp rate of $3^{\circ} \mathrm{C} / \mathrm{min}$, all hydrolysate samples displayed a shorter $t_{g e l}$ and lower $T_{\text {gel }}$ than SPI at the same concentration, which suggested that enzymatic proteolysis could shorten the onset of gelation of soy protein no 
matter which component is hydrolyzed. Under similar DH, DGH showed earlier gelling points than their $\mathrm{D} B \mathrm{H}$ counterparts, which can be explained by the fact that glycinin is more thermally stable and has a higher denaturation temperature than $\beta$-conglycinin [49]. As a result, selectively hydrolyzing glycinin could facilitate the process of thermal denaturation and gelation of soy protein to a larger extent. Increasing the $\mathrm{DH}$ significantly decreased $\mathrm{t}_{\text {gel }}$ and $T_{\text {gel }}$ of $\mathrm{D} \beta \mathrm{H}$, and dialysis further decreased these values (except for $14 \% \mathrm{D} \beta \mathrm{H}-\mathrm{L} / \mathrm{D}$ ). However, these trends were not detectable among DGH samples. DGH solutions were found quite viscous before gelation, which brought more difficulties in determining their accurate gelling points.

Except for differences in the gelling points, the gelation process at constant high temperature $\left(95^{\circ} \mathrm{C}\right.$ ) was also found to be different among these samples, as was apparent from the average slope of the curves of $\mathbf{G}^{\prime}\left(\mathbf{S}_{\mathbf{\Delta} \mathbf{G}^{\prime}}\right)$ versus time, in this isothermal period (Table 4.2). The $S_{\triangle G^{\prime}}$ of $D \beta H-L / N D$ was higher than that of SPI while the $S_{\triangle G^{\prime}}$ of $D G H-L / N D$ was significantly lower. Increasing the $\mathrm{DH}$ decreased the $\mathrm{S}_{\triangle \mathrm{G}^{\prime}}$ of $\mathrm{D} \beta \mathrm{H}-\mathrm{H} / \mathrm{ND}$ but did not affect the $\mathrm{S}_{\triangle \mathrm{G}^{\prime}}$ of $\mathrm{DGH}-$ $H / N D$. These results indicated that limited hydrolysis of $\beta$-conglycinin can contribute to the stiffening process of soy protein gels during heating, while hydrolyzing glycinin significantly hinders this process. Since glycinin is the component that has the highest surface hydrophobicity and number of disulfide groups in soy protein, the interactions during heating would be weaker in DGH as glycinin was hydrolyzed into small peptides. These small peptides in the hydrolysates do not appear to be incorporated in the gel network during thermal gelation, as all $\mathrm{S}_{\Delta \mathrm{G}^{\prime}}$ increased after dialysis. 


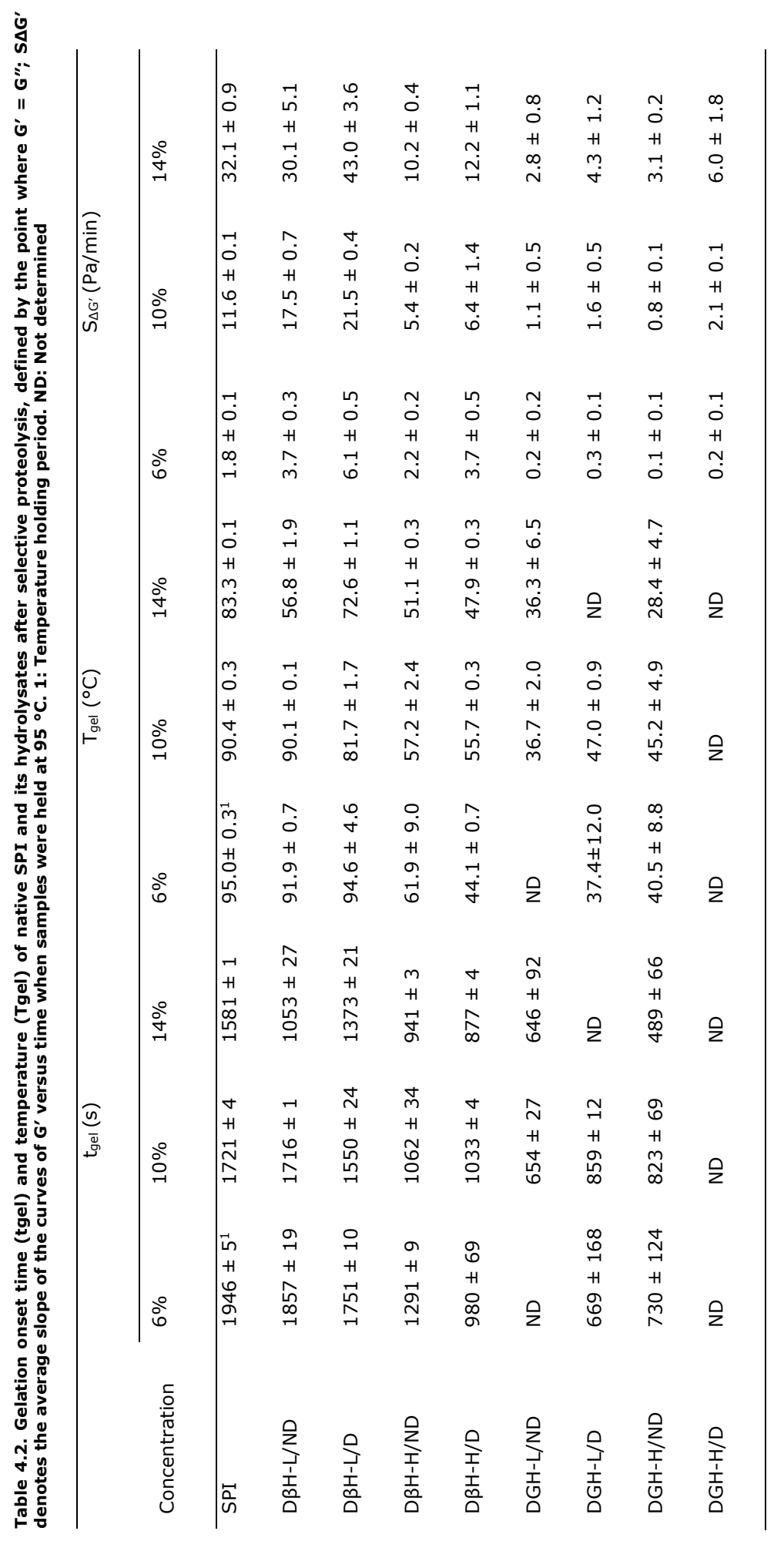




\section{Linear viscoelastic (LVE) properties of heat-set gels}

Fig. 4.5 shows the $\mathrm{G}^{\prime}$ of different gels in the LVE regime as a function of protein concentration. All $D B H$ gels showed a higher $\mathrm{G}^{\prime}$ than SPI at $6 \%$ protein concentration (Fig. $4.5 \mathrm{~A})$, suggesting that hydrolyzing $\beta$-conglycinin not only shortened the onset of gelation but also improved the gel stiffness at lower protein concentrations. This makes $D \beta H$ promising for application in meat-replacement products like sausages, which benefit from proteins that form stronger gels at lower concentration and temperature. As the protein concentration increased, the $G^{\prime}$ of $D B H$ gels was gradually exceeded by the $G^{\prime}$ of SPI. This difference in the slope of the $\mathrm{G}^{\prime}$ vs. concentration curve suggests that selective proteolysis of $\beta$-conglycinin affects the microstructure of the gels. When the concentration reached $14 \%$, only DBH-L/D showed a higher $\mathrm{G}^{\prime}$ than the SPI gel. Note that in the dialyzed samples, the small peptides have been removed, and these samples therefore contain a relatively higher amount of glycinin. All DGH gels, on the other hand, showed significantly lower G' than SPI gels at every concentration (Fig. 4.5B), showing that hydrolyzing glycinin significantly reduced the stiffness of soy protein gels.
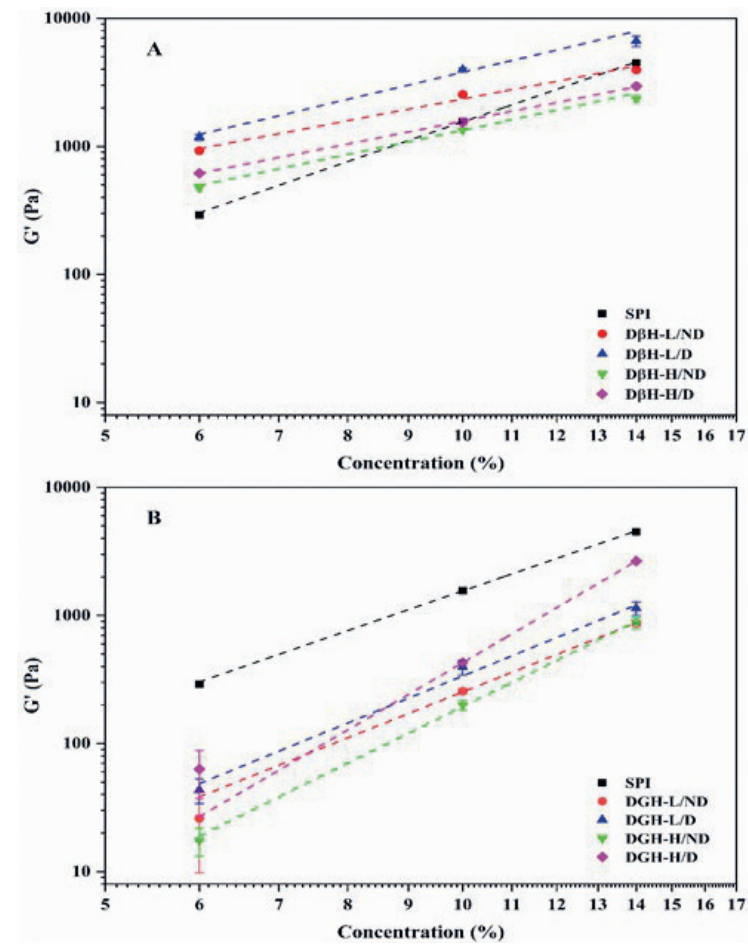

Figure 4.5. $G^{\prime}$ obtained from the LVE region of the strain sweep (performed after completion of the temperature cycle) as a function of protein concentration for native SPI and its hydrolysates after selective proteolysis of $\beta$-conglycinin $(A)$ and glycinin (B). 
Our results agreed with previous studies $[10,128,155,156]$, which reported that at the same $\mathrm{pH}$ and protein concentration, a higher glycinin to $\beta$-conglycinin ratio led to stiffer and harder tofu or soy protein gels. Pavlicevic et al. attributed this phenomenon to the high content of sulfhydryl groups in the acidic polypeptides of glycinin and the stabilization of gels via disulfide bonding, which is consistent with the higher stiffening rate (higher $S_{\triangle G^{\prime}}$ ) of $D \beta H$ samples during heating (Table 4.2) [157]. In addition, Wu et al., found the higher $\mathrm{G}^{\prime}$ was also related to the larger and compacter aggregates formed by the basic polypeptides of glycinin [128], which can be verified by examining the microstructure of DBH gels (Fig. 4.9). Tsumura et al. reported the opposite, that gels formed by pepsin-treated SPI (similar to DGH) showed higher $G^{\prime}$ than gels formed by papain-treated SPI (similar to D $B H$ ) [31]. The exact reason behind this contradiction remains unclear but might be related to different gelation conditions, such as ionic strength. We observed that the ionic strength of different hydrolysates is significantly different (data not shown), so we standardized the conductivity as well as the $\mathrm{pH}$ of samples before gelation.

Gels formed by $D \beta H-H / N D$ and $D G H-H / N D$ showed lower $G^{\prime}$ than the gels formed by $D \beta H-$ L/ND and DGH-L/ND, indicating that no matter which component is hydrolyzed, extensive hydrolysis can be an impairment for the gel stiffness $[136,158]$. Gels formed by dialyzed samples (DBH-L/D, D $B H-H / D, D G H-L / D$, and DGH-H/D) showed higher $G^{\prime}$ than the gels formed by their non-dialyzed counterparts (D $\beta H-L / N D, D \beta H-H / N D, D G H-L / N D$, and DGH$\mathrm{H} / \mathrm{ND}$ ). Wu et al. reported that the protein fraction with MW up to 9.7 kDa was a non-network protein during the heat-induced gelation of soy protein [159]. Thus, a lower fraction of nonnetwork protein in the dialyzed samples leads to stiffer heat-set gels [16]. Although it is likely that the peptides resulting from selective proteolysis are not incorporated in the gel, based on the above observations, the removal of these small protein fractions does affect the microstructure and strength of the hydrolysate gels. The tan $\delta$ values of gels ranged from 0.120 to 0.237 (see Table S4.1), indicating all gels behaved more elastic than viscous $\left(G^{\prime}>G^{\prime \prime}\right)$ in the LVE regime. Hydrolysate gels showed a higher tan $\delta$ than SPI gels, indicating that although the response was still more elastic than viscous, proteolysis induced a small increase in the relative importance of the viscous contribution to the response.

Table 4.3 shows the power-law parameters of the relation between the $\mathrm{G}^{\prime}$ of gels and protein concentration $C$, i.e., $G^{\prime} \propto C^{A}$. The $R^{2}$ of the fitted lines were all above 0.98 . $\mathrm{D} \beta \mathrm{H}$ gels showed a lower value for the exponent $A$, ranging from 1.75 to 2.19 , while DGH gels showed a higher value which ranged from 3.70 to 5.42 . The value for $A$ of SPI gel was in between these two ranges, equal to about 3.20. Similarly, Renkema et al. found that heat-set gels ( $\mathrm{pH} 7.6,0.2$ $\mathrm{M} \mathrm{NaCl}$ ) formed by $\beta$-conglycinin rich fractions showed higher values for $A$ (4.6) than the gels formed by purified glycinin (3.5) [10]. A higher value for the exponent $A$ indicated that the protein in DGH gels was used less efficiently and was distributed more heterogeneously, 
which is consistent with their microstructure as shown later (Fig. 4.8). Shih et al. used fractal theory to describe the structure of gel networks, and introduced a fractal dimension $\left(\boldsymbol{D}_{\boldsymbol{f}}\right)$ of the gel structure, estimated in two regimes, i.e. the strong-link and weak-link regimes [26]. In the strong-link regime, the inter-floc links are stronger than the intra-floc links. In this regime $A=(3+x) /\left(3-D_{f}\right)$, where $x$ is the fractal dimension of the backbone of the flocs, which is assumed to be 1.1 in the present study. In the weak-link regime, the flocs are more rigid, and the inter-floc links are weaker, and $A=1 /\left(3-D_{f}\right)$. Wu and Morbidelli further developed the scaling theory of Shih et al. by introducing a scaling relation between critical linear strain $\left(y_{c}\right)$ and protein concentration i.e. $\gamma_{c} \propto C^{B}$ [27]. They show that the value for the exponent $B=(1-[2+x][1-a]) /\left(3-D_{f}\right)$, where the parameter $a$ equals zero in the strong-link regime, is equal to 1 in the weak-link regime, and $0<a<1$ in the transition region. As shown later in the next section, except for the SPI gels, most gels in this study displayed negligible changes in the $\gamma_{c}$ with different protein concentrations, therefore we could not use this scaling relation to determine $a$, and hence determine which regime the gel was in. To overcome this problem, we calculated the $D_{f}$ of the gels based only on the value of $A$, as previous studies did $[160,161]$. We used the expressions for both regimes, and then chose the $D_{f}$ value that was most physical and realistic, according to the normal range of $D_{f}$ known for protein gels, i.e., 1.7 to 2.4 .

As shown in Table 4.3, the scaling of $\gamma_{c}$ proposed by Wu and Morbidelli could only be applied for SPI gels, which clearly showed a decrease of $\gamma_{c}$ with increasing concentration. The $D_{f}$ of SPI gels using this model is 2.45 , and the $a$ is 0.75 , indicating that the SPI gel is in the transition regime and close to the weak-link regime. For $D \beta H$ gels, the strong-link model gave $D_{f}$ values about 1 or even lower, which neither has any physical meaning nor agrees with their microstructures (Fig. 4.9). The weak-link model gave rather realistic $D_{f}$ values around 2.5. For DGH gels, the weak-link model gave $D_{f}$ values above 2.7 , which were rather high for gels formed at only $6 \%$ to $14 \%$ protein concentration, while the strong-link model gave more realistic $D_{f}$ values ranged from 1.89 to 2.24 . In conclusion, the $D_{f}$ values derived by the scaling theory of Shih et al. indicated that $\mathrm{D} \beta \mathrm{H}$ gels belong to the weak-link regime while DGH gels are close to the strong-link regime. The results are consistent with the finding of Wu and Morbidelli that the $D_{f}$ increases as the gel changes from strong-link type (DGH) to transition type (SPI) to weak-link type $(D \beta H)$. All the distinct changes in the SAOS tests discussed above imply that the heat-set gels formed by native SPI and these hydrolysates should also respond differently in LAOS tests, which will be addressed in the next section. 


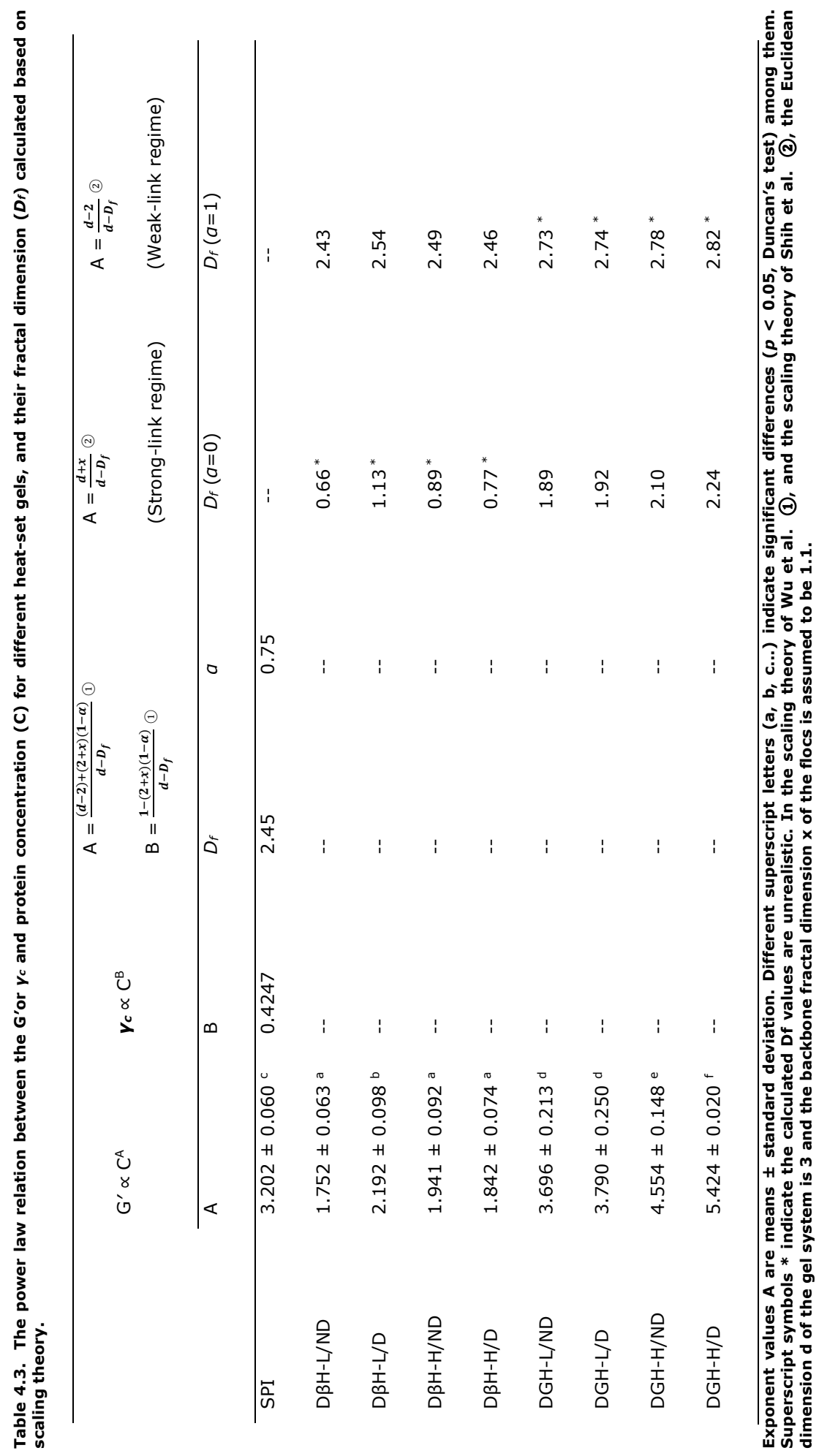




\subsubsection{Non-linear rheology}

\section{Intercycle strain dependence of $G^{\prime}$ and $G^{\prime \prime}$}

Amplitude-sweep tests were used to determine $\mathrm{G}^{\prime}$ and $\mathrm{G}^{\prime \prime}$ of different gels as a function of $Y_{0}$ (from $1 \%$ to $1000 \%$ ), at $1 \mathrm{~Hz}$ and $20^{\circ} \mathrm{C}$. As shown in Fig. 4.4D-F and Fig. S4.2, all gels had a clearly observable LVE regime, and the limit of this regime i.e. $\gamma_{c}$ was estimated by the strain where the deviation of $\mathrm{G}^{\prime}$ from its constant value in the LVE reached 5\% [162]. As shown in Table 4.4, at the same concentration SPI gels had much higher $\gamma_{c}$ than hydrolysate gels $(p<0.05)$, which indicated that proteolysis made the soy protein gels more brittle. As the protein concentration increased, the $\gamma_{c}$ of SPI gels further increased, while the $\gamma_{c}$ of hydrolysate gels showed no concentration dependency, except DBH-L/ND gels, which showed a mildly decreasing trend.

Heat-set gels formed by D $\beta H-L / N D$ (Fig. S4.2), D $\beta H-H / N D$ (Fig. 4.4E) and their dialyzed counterparts (Fig. S4.2) showed a smoothly decreasing $\mathrm{G}^{\prime}$ and $\mathrm{G}^{\prime \prime}$ upon increasing yo beyond the LVE regime. According to the classification of Hyun et al., D $\beta H$ gels displayed strain softening (Type I) LAOS behavior, which is generally related to a decreased network-bond creation rate and an increased loss rate with increased yo [24]. This behavior agreed with the above analysis by scaling theory, which indicated that $D \beta H$ gels are weak-link gels. Since the constituted flocs are interconnected weakly, once they dissociated under large deformation, they tend to align with the flow field, leading to progressive softening. These limited interactions between flocs can be explained by the fact that during the gelation of $\mathrm{D} \beta \mathrm{H}$, the flocs were formed by aggregation of glycinin molecules that were only unfolded moderately and still largely globular [163]. This rigid structure of protein particles suppresses the intermolecular crosslinking between flocs while favoring the intramolecular bonding.

For the gels formed by DGH-L/ND (Fig. S4.2), DGH-H/ND (Fig. 4.4F) and their dialyzed counterparts (Fig. S4.2), the $\mathrm{G}^{\prime}$ also decreased smoothly as $y_{0}$ increased, but at higher DH and at higher concentration a mild overshoot in $\mathrm{G}^{\prime \prime}$ was observed. This is defined as weak strain overshoot (Type III) behavior [139], which is found when the bond creation rate and loss rate both increase with $y o$, while the latter one grows faster. Compared to glycinin, the MW of $\beta$-conglycinin is smaller and the molecular structure is known to be more flexible [164]. As a result, the flocs formed by the $\beta$-conglycinin in DGH could be more disordered and branched, and could be interconnected with each other more strongly. Upon increased strain, flocs that dissociated initially were likely to get entangled to reform new clusters, giving rise to shear thickening behavior (increased $G^{\prime \prime}$ ). When the deformation further 
increased, the backbone structure of flocs was disrupted completely, leading to a decrease of $\mathrm{G}^{\prime}$ and $\mathrm{G}^{\prime \prime}$ eventually.

Finally, SPI gels displayed increasing $\mathrm{G}^{\prime}$ and $\mathrm{G}^{\prime \prime}$ at intermediate strain amplitude before decreasing (Fig. 4.4D), which is defined as strong strain overshoot (Type IV) LAOS behavior [139]. This behavior indicates that the interactions within the SPI gel network are likely to be stronger than for DGH gels (Type III), in which only G" showed a mild overshoot. As mentioned earlier, SPI gels belong to an intermediate system, in which both inter- and intrafloc links contribute to the overall elasticity. This Type IV behavior was found in gelled polystyrene latex by Gisler et al, who attributed this strain hardening in fractal colloidal gels to the intrinsic stiffness of contorted backbone segments; increasing strain straightens these segments, resulting in an increase in the $\mathrm{G}^{\prime}$ as well [165].

Table 4.3. Critical linear strain $(Y c)\left(1 \mathrm{~Hz}\right.$ and $\left.20^{\circ} \mathrm{C}\right)$ of the heat-set gels formed by native SPI and its hydrolysates after selective proteolysis, defined by the point where the deviation of $G^{\prime}$ from its constant value in the LVE reached $5 \%$.

\begin{tabular}{|c|c|c|c|}
\hline \multirow[b]{2}{*}{ Protein concentration } & \multicolumn{3}{|c|}{$Y_{c}(\%)$} \\
\hline & $6 \%$ & $10 \%$ & $14 \%$ \\
\hline SPI & $28.05 \pm 3.75^{d}$ & $32.30 \pm 2.97^{\mathrm{e}}$ & $40.65 \pm 0.78^{f}$ \\
\hline DBH-L/ND & $7.59 \pm 1.46^{b c}$ & $5.96 \pm 0.70^{b}$ & $2.65 \pm 0.17^{a}$ \\
\hline DßH-L/D & $8.00 \pm 0.49 \mathrm{bc}$ & $9.93 \pm 4.19^{c}$ & $5.91 \pm 2.01^{b}$ \\
\hline DßH-H/ND & $2.75 \pm 0.34^{a}$ & $3.53 \pm 0.41^{a}$ & $3.01 \pm 0.25^{a}$ \\
\hline DßH-H/D & $2.51 \pm 0.02^{a}$ & $2.42 \pm 0.08^{a}$ & $2.38 \pm 0.23^{a}$ \\
\hline DGH-L/ND & $2.32 \pm 0.07^{a}$ & $3.07 \pm 0.20^{a}$ & $3.31 \pm 0.47^{a}$ \\
\hline DGH-L/D & $2.86 \pm 0.35^{a}$ & $3.16 \pm 0.65^{a}$ & $3.12 \pm 0.11^{a}$ \\
\hline DGH-H/ND & $2.61 \pm 0.08^{a}$ & $2.70 \pm 0.17^{a}$ & $2.52 \pm 0.28^{a}$ \\
\hline DGH-H/D & $3.29 \pm 0.08^{a}$ & $2.68 \pm 0.19^{a}$ & $3.22 \pm 1.04^{a}$ \\
\hline
\end{tabular}




\section{Lissajous plots}

Since $\mathrm{G}^{\prime}$ and $\mathrm{G}^{\prime \prime}$ are based only on the first harmonic contribution to the stress response and may have ambiguous physical meaning in the NLVE regime [139], the full oscillatory response at each imposed yo was analyzed using Lissajous plots. Fig. 4.6A shows the normalized Lissajous plots of shear stress $(\sigma)$ vs. intracycle strain $(\gamma)$, referred to as elastic Lissajous plots, for the gels formed at $10 \%(\mathrm{w} / \mathrm{w})$. The enclosed area of elastic Lissajous plots is related to the dissipated energy (proportional to $\mathrm{G}^{\prime \prime}$ ), and the decomposed elastic stress $\left(\sigma_{e}\right)$ in the interior of the loop indicates the purely elastic contribution to the total shear stress $(\sigma)$.

When $y_{0}$ was equal to $6.34 \%$, all elastic Lissajous plots showed a narrow and elliptical shape, and $\sigma_{e}$ was a straight line, which is typical for LVE behavior. The SPI and D $\beta H$ gels showed significant narrower ellipses, i.e. a significantly lower dissipated energy per cycle, than the DGH gels, which well agreed with the SAOS results that SPI and D $B H$ gels showed significant higher $\mathrm{G}^{\prime}$ (Fig.4.5) and lower tan $\delta$ than DGH gels (Table S4.1). As $\gamma_{0}$ increased to $25.2 \%$, the Lissajous plot of SPI remained narrow and nearly elliptical. In contrast, the Lissajous plots of hydrolysate gels became distorted, reflecting the impact of higher harmonics. This demonstrated that hydrolysate gels were well into their non-linear viscoelastic (NLVE) regime while the SPI gel was not, which is consistent with its highest $\gamma_{c}$ among all the gels studied here (Table 4.4). The distortion of the Lissajous plots is of different nature and magnitude. For $\mathrm{D} \beta \mathrm{H}-\mathrm{L} / \mathrm{ND}$ and $\mathrm{D} \beta \mathrm{H}-\mathrm{L} / \mathrm{D}$ gels, the Lissajous plots only show a slight upswing, indicating a minor intracycle strain stiffening effect. The differences between the loop of $\sigma$ and $\sigma_{\mathrm{e}}$ remained relatively small. These gels were the only hydrolysate gels that displayed a higher $\mathrm{G}^{\prime}$ than SPI at $10 \%$ concentration (Fig. 4.5). For the other hydrolysate gels, the Lissajous plots changed to a near rhomboidal shape, and the enclosed area expanded significantly, which suggested significant structure breakdown [150].

As yo increased to $101 \%$, the SPI gel showed a narrow and inverted sigmoidal Lissajous plot. The steeper slope at higher intracycle strain reflects the stretching of clusters, causing strong intracycle stiffening [166]. This distinct upswing was also observed in the total stress for $\mathrm{D} \beta \mathrm{H}$ gels, where in view of the structural breakdown, it is related to the stretching of remaining clusters. For DGH gels, this upswing is discernable only in the elastic contribution when the maximum intracycle strain is approached. Besides, their Lissajous plots had already changed into an almost square shape and did not change too much even when $y_{0}$ further increased, which meant the decay of elasticity was much faster in DGH gels.

When yo was equal to $201 \%$, all Lissajous plots showed a rhomboidal shape. Starting from the lower-left corner, an intracycle sequence of elastic straining (the vertical part), yielding, 
flow (the nearly horizontal part), and (partial) recovery are observed. The Lissajous plot of $\mathrm{D} \beta \mathrm{H}-\mathrm{L} / \mathrm{ND}$ gel showed a smaller enclosed area than that of $\mathrm{D} \beta \mathrm{H}-\mathrm{H} / \mathrm{ND}$ gel. Furthermore, $D \beta H-L / D$ and $D \beta H-H / D$ gels showed narrower Lissajous plots than their non-dialyzed counterparts, i.e., $D \beta H-L / N D$ and $D \beta H-H / N D$ gels. These results suggested that hydrolyzing $\beta$-conglycinin to a higher $\mathrm{DH}$ and generating small peptides gave the gels a relatively more viscous behavior. However, these differences were not discernable among DGH gels.

At the largest $y_{0}$, i.e., $1010 \%$, all Lissajous plots changed into a nearly rectangular shape, indicating all gels had changed from predominantly elastic to almost perfect plastic behavior $[150,167]$. This rectangular shape has also been observed in the LAOS tests of other food materials such as debranched waxy rice starch gels [147], pea protein gels [134], and concentrated wheat gluten [150]. For the SPI and D $3 H$ gels, the Lissajous plots showed sharper and straighter corners, indicating a more abrupt intracycle yielding behavior. On the other hand, for the DGH gels, their Lissajous plots showed smoother and more rounded corners, reflecting a more gradual yielding behavior.

Fig. 4.6B shows the normalized viscous Lissajous plots of $\sigma$ vs. intracycle strain rate $(\dot{\gamma})$, for gels formed at $10 \%(\mathrm{w} / \mathrm{w})$. In contrast to the elastic Lissajous plots, the enclosed area of viscous Lissajous plots is related to the stored energy (proportional to $\mathrm{G}^{\prime}$ ), and the red dashed line in the middle indicates the contribution of the viscous stress $\left(\sigma_{v}\right)$ to $\sigma$. When $y_{0}$ was $6.34 \%$, all gels displayed circular plots for $\sigma$ and straight lines for $\sigma_{v}$, which agreed with the predominantly elastic behavior in the LVE regime. As $Y_{0}$ increased, the enclosed area of the viscous Lissajous plots decreased in the order of DGH gels $<$ D $\beta H$ gels $<$ SPI gels. When Y was higher than $101 \%$, all viscous plots had a sigmoidal shape, indicating intracycle shear thinning behavior, due to the breakdown of the network structure and alignment of segments in the direction of the flow.

At the largest $y_{0}(1010 \%)$, secondary loops were clearly observed in the viscous plots of SPI and DGH gels. This particular shape is an indicator of an overshoot in the stress, which could be associated with their strain overshoot (Type II and Type IV) LAOS behavior. These stress overshoots can have multiple origins. One possible reason for their occurrence is a coupling of the elasticity of the sample with instrument inertia [147]. Such patterns also emerge when the time scale for restructuring of the microstructure is shorter than the time scale of the deformation [168]. Here for SPI, the rather irregular secondary oscillations (wave-like horizontal part) observed in the elastic Lissajous plot at $1010 \%$ strain (Fig. 4.6A) leads us to believe that elasticity-inertia coupling is a more likely explanation. 
Figure 4.6. Normalized Lissajous plots of total stress $(\sigma)$ (solid line) vs. strain (Y) (A) or strain rate ( $\dot{Y})(B)$ for the heat-set gels formed by $10 \%(w / w)$ of (from left to right) SPI, D $3 H-L / N D, D \beta H-L / D, D \beta H-H / N D, D \beta H-H / D$, DGH-L/ND, DGH-L/D, DGH-H/ND, and DGH-H/D, with intercycle strain amplitude ( $(y)$ of $6.34 \%, 25.2 \%$, $101 \%, 201 \%$ and $1010 \%$, at $1 \mathrm{~Hz}$ and $20 \% \mathrm{C}$. Dashed line in the interior of the loop indicates the decomposed elastic stress (A) and viscous stress (B).

(A)

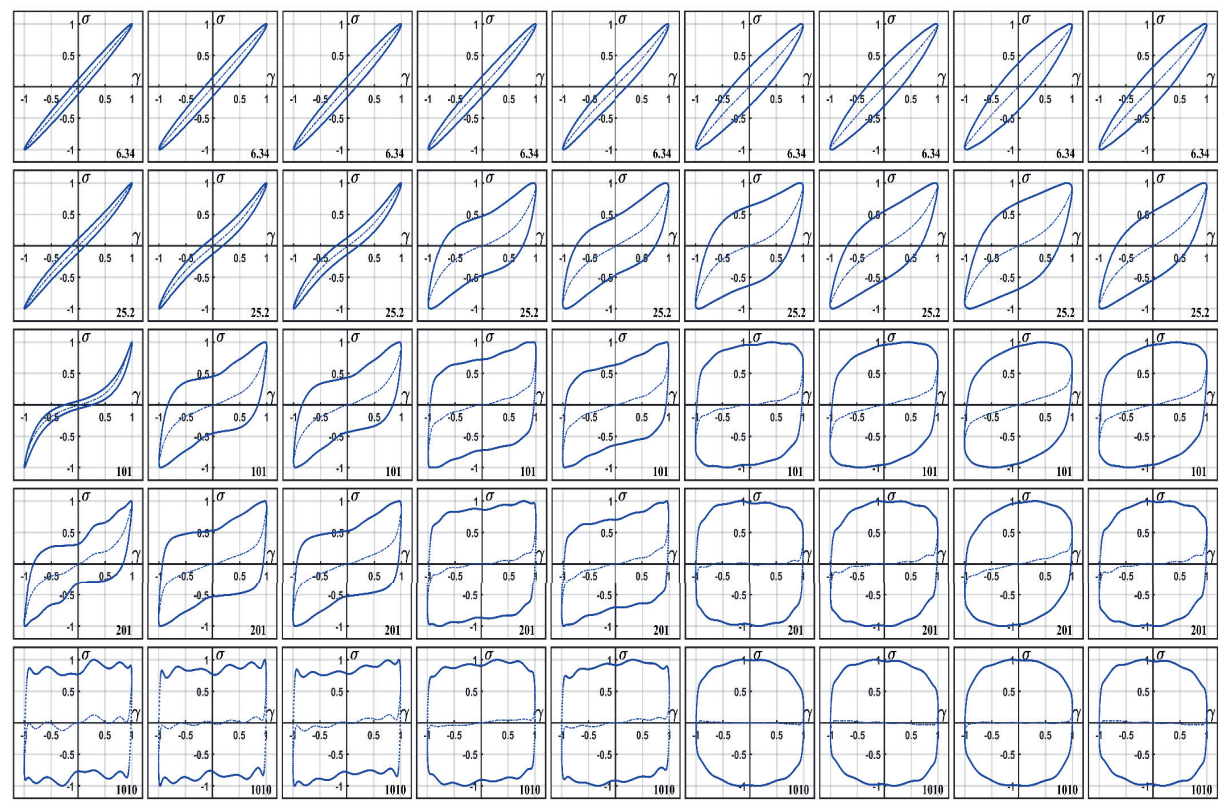

(B)

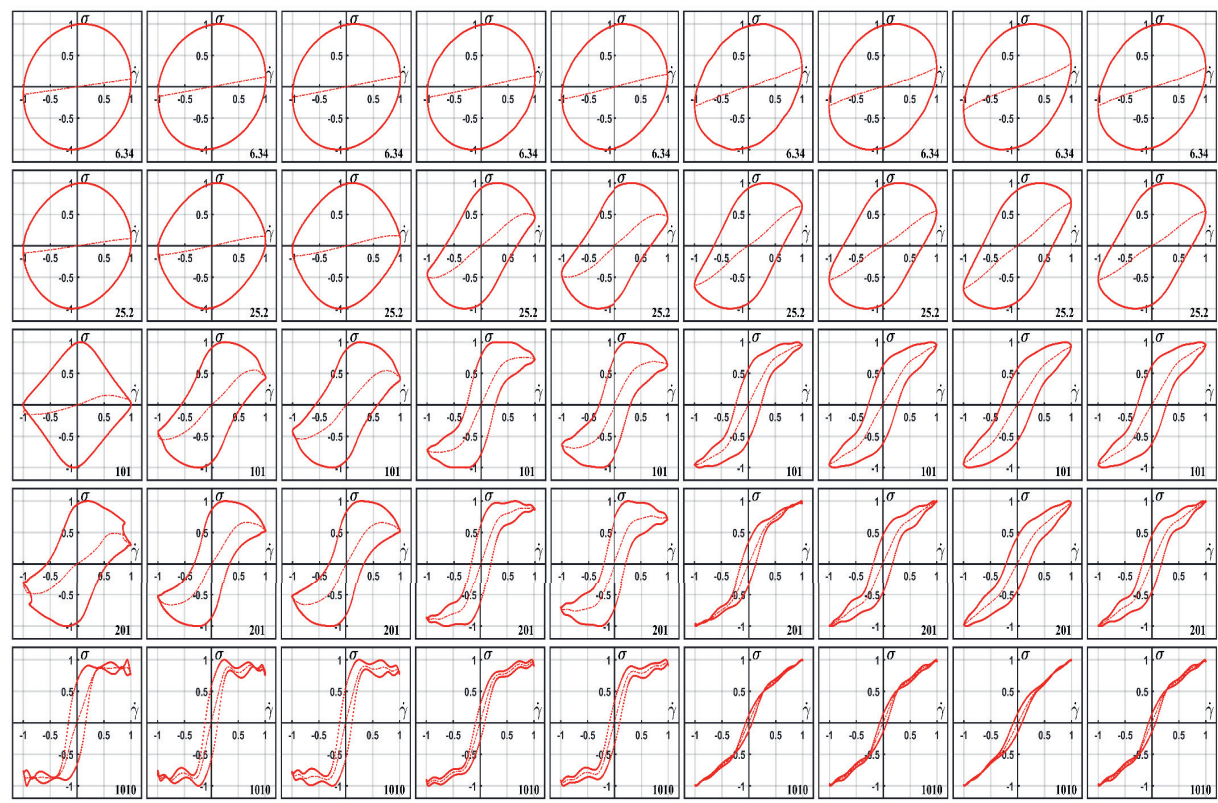


The non-linear rheological behavior was further quantified by calculating the S-factor and Tfactor (Fig. 4.7). These factors describe the intracycle behavior at a fixed $y_{0}$, which should not be confused with the overall intercycle behaviors shown in Fig. 4.4D-F. For all gels, the $\mathrm{S}$-factor was positive over the entire NLVE regime, which is consistent with the upswing (intracycle stiffening behavior) observed in all elastic Lissajous plots (Fig. 4.6A). Compared to SPI gels, hydrolysate gels displayed higher S-factors, but this was the result of an apparent stiffening effect, caused by the near zero slopes at minimal strain $G_{M}^{\prime}$, and large secant modulus $G_{L}^{\prime}$. Given the very narrow elastic Lissajous plot and strong upswing, SPI gels actually showed "real" stiffening when the yo was around $101 \%$. The curves of the Sfactor all show a decrease in slope in the middle range of strain amplitude followed by a second increase, especially for SPI gels. Generally, positive S-factors indicate strain stiffening behavior, but they may also indicate a transition from elastic to plastic behavior. Combined with the Lissajous plots, the initial increase in the $\mathrm{S}$-factor curves is related to intracycle strain stiffening effects, while the second increase at higher yo could be ascribed to the prevailing plastic behavior.

The T-factor was predominantly negative for $\mathrm{DBH}$ gels in the entire range of intercycle strain rate $\left(\dot{\gamma}_{0}\right)$ and the value was significantly lower than that for SPI and DGH gels, reflecting a stronger intracycle shear thinning behavior in $\mathrm{D} \beta \mathrm{H}$ gels. In line with our previous analysis, once flocs in $\mathrm{D} \beta \mathrm{H}$ gels were disassociated, they were less likely to reconnect under shear flow. For SPI and DGH gels, the T-factor was initially positive and increased with $\dot{\gamma}_{0}$, and subsequently decreased and became negative when $\dot{\gamma}_{0}$ exceeded about $2.4 \mathrm{~s}^{-1}$. This phenomenon indicated that with increasing $\dot{\gamma}_{0}$, SPI and DGH gels first showed intracycle shear thickening followed by shear thinning behavior, which is consistent with the overshoot phenomena observed in their G" (Fig. 4.4F). 

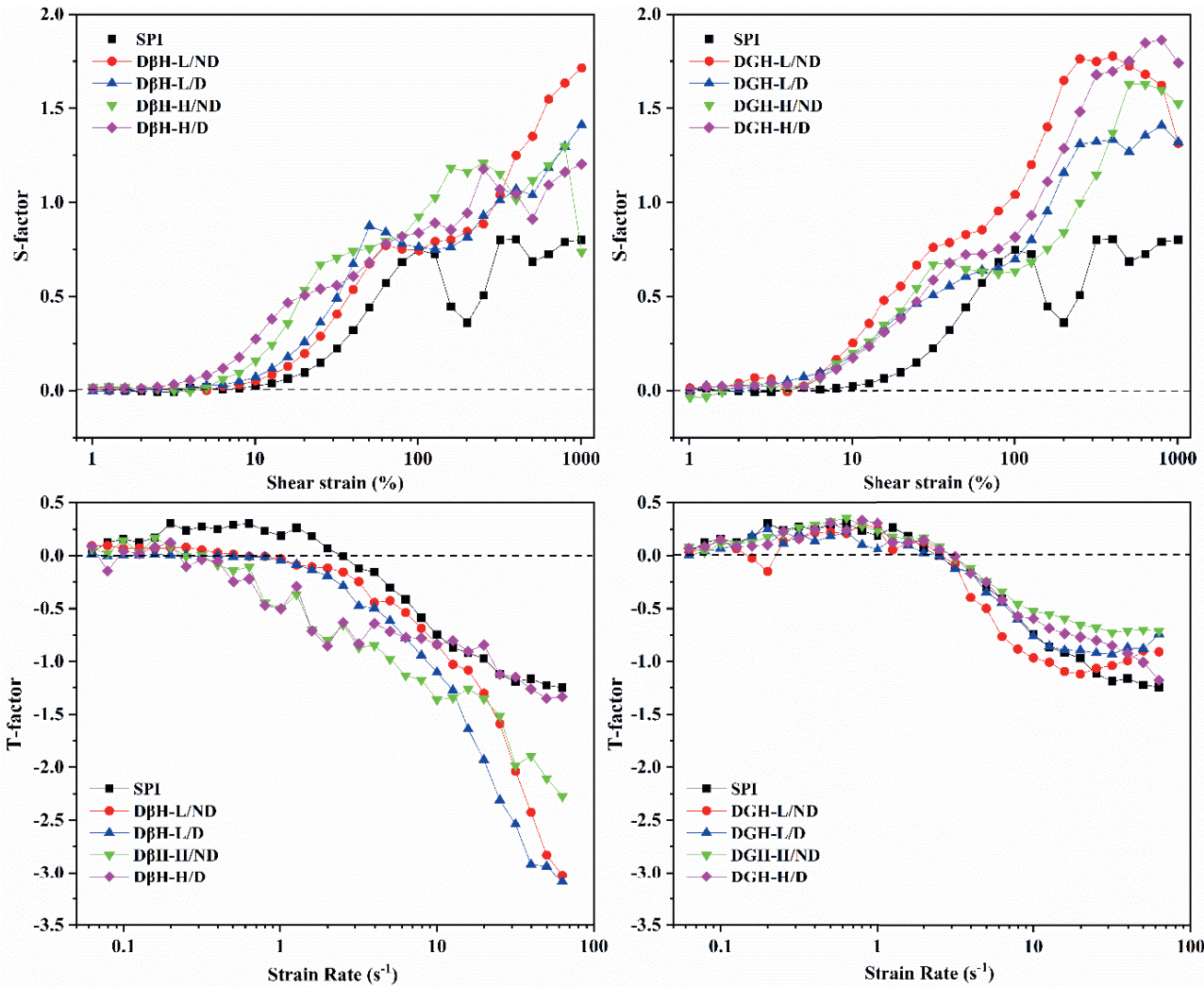

Figure 4.7. Ratio of shear stiffening (S-factor) and ratio of shear thickening (T-factor) for SPI, D $\beta H$ and DGH heat-set gels.

\subsubsection{Microscopy of soy protein gels}

Fig. 4.8 shows the microstructures of gels formed at $10 \%(\mathrm{w} / \mathrm{w})$, visualized by CLSM and SEM. In the CLSM images, SPI gels showed a coarse and heterogeneous structure with relatively large pores, some of which are indicated by green arrows (Fig. 4.8A). With SEM, the microstructure of the gels was observed at a much smaller length scale $(3 \mu \mathrm{m})$. An aggregated gel structure was clearly observed for SPI (Fig. 4.8a), which is typical for heatset globular protein gels [169]. Upon heating a globular protein solution, the protein molecules were denatured and associated with each other by non-covalent and covalent interactions to form flocs. These flocs associate further to form a space spanning 3D gel network [170]. After selectively hydrolyzing $\beta$-conglycinin, the gel structure of $D \beta H-L / N D$ appeared more homogenous using CLSM, and only a few pores with significantly smaller sizes were observed (Fig. 4.8B). After dialysis, the gel structure of D $\beta H$-L/D was more compact, where pores were hard to distinguish without further magnification. In addition, protein aggregates appeared to associate into clumps, indicated by green circles (Fig. 4.8C). 
The results demonstrated that hydrolyzing $\beta$-conglycinin to a limited extent could increase the density of SPI gel structure. Dialysis not only removed the non-network peptides, but also increased the relative content of glycinin, favoring the formation of protein aggregates and an even denser gel network. This finer microstructure of D $B H-L / N D$ and $D \beta H-L / D$ gels explained their comparable or even higher $\mathrm{G}^{\prime}$ compared to SPI gels as revealed by SAOS tests. Increasing the $\mathrm{DH}$ made the pores appear again in the gel microstructure of $\mathrm{D} \beta \mathrm{H}$ $\mathrm{H} / \mathrm{ND}$ (Fig. 4.8D), which clearly demonstrated that extensive hydrolyzing $\beta$-conglycinin has negative effects on the density and continuity of the gel network. Similarly, after dialysis, these pores become fewer and smaller in D $\beta H-H / D$ gel (Fig. 4.8E). SEM also revealed an aggregated gel structure for $D \beta H$ gels, but the flocs in $D \beta H$ gels were more compact and were formed by larger globular aggregates (Fig. 4.8b-e). The high structural rigidity of these flocs contributed to the higher stiffness of $D \beta H$ gels, but also led to weak inter-floc links which was manifested in the strong shear thinning and brittle yielding behavior in LAOS tests.

In contrast, selectively hydrolyzing glycinin made the gel structure of DGH-L/ND and DGH$\mathrm{H} / \mathrm{ND}$ much coarser and discontinuous (Fig. 4.8F, H), with the existence of larger pores and big cracks (indicated by green arrows), which is consistent with their low $D_{f}$ derived from scaling theory (Table 4.3). Several dark faint red areas were also observed within the network (indicated by blue arrows), which we attribute to syneresis. Generally, the permeability of gels increases and the ability to retain water decreases when the network structure coarsens $[16,171]$. After dialysis, the gel structure of DGH-L/D and DGH-H/D was relatively denser and compact (Fig. 4.8G, I), but a lot of protein macroaggregates, with different sizes and shapes, appeared in the gel network (indicated by green circles). Under SEM, DGH gels displayed a more heterogeneous and complicated structure (Fig. 4.8f-i), which was clearly different from SPI gels and DBH gels. The flocs are thinner and smaller but more branched and intertwined. By transmission electron microscopy, Hermansson (1985) also observed that $\beta$-conglycinin rich gels were less regular and more crosslinked, and the strands or flocs formed by $\beta$-conglycinin molecules showed a complex mode of aggregation, possibly in the form of double spirals [172]. These structural features not only caused DGH gels to be less stiff and have a relatively more viscous rheological response, but also caused them to be classified in the strong-link regime, as the scaling theory indicated (Table 4.3). Under shear deformation, these flocs are more likely to re-entangle with each other after being dissociated, which well explains the weak strain overshoot (Type III) behavior and the gradual yielding behavior that DGH gels showed in the LAOS tests. 
Figure 4.8. Confocal laser scanning microscopy (left, magnification $20 \times$ ) and scanning electron microscopy (right, magnification $25000 \times$ ) of gels formed by $10 \%(w / w)$ of SPI, D BH-L/ND, D $\beta H-L / D, D \beta H-H / N D, D \beta H-$ H/D, DGH-L/ND, DGH-L/D, DGH-H/ND, and DGH-H/D (from top to bottom).
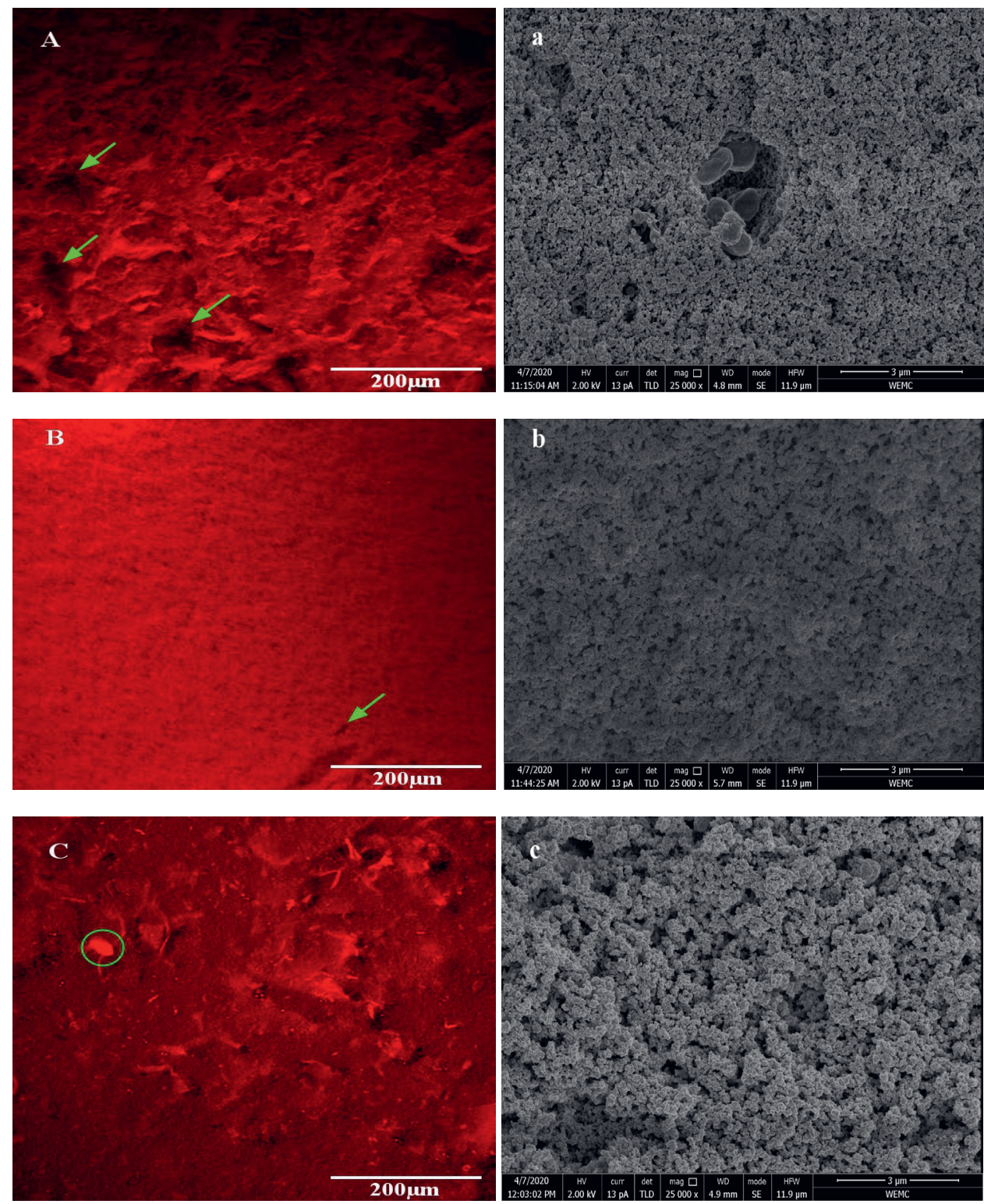
Figure 4.8. (continued)
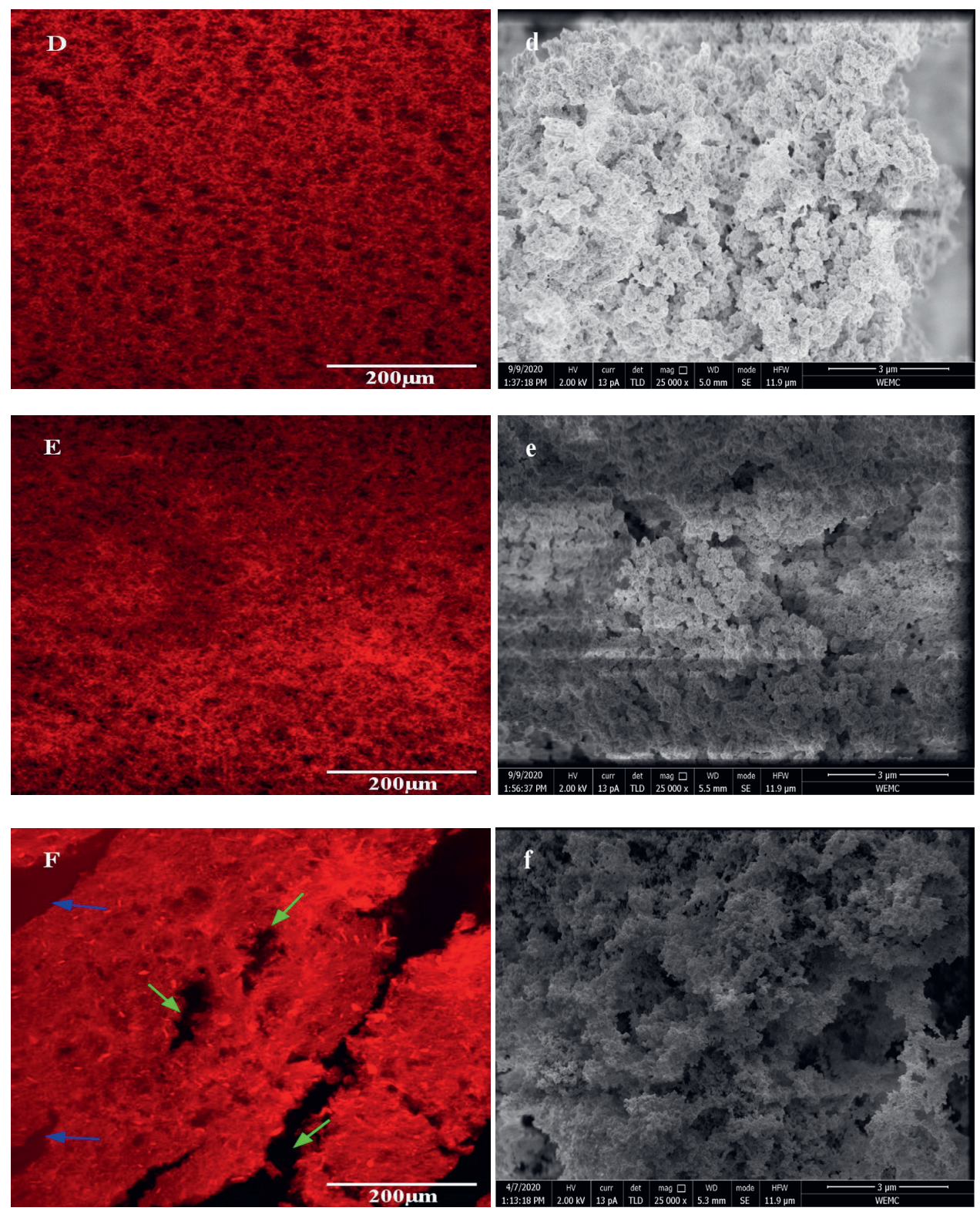
Figure 4.8. (continued)
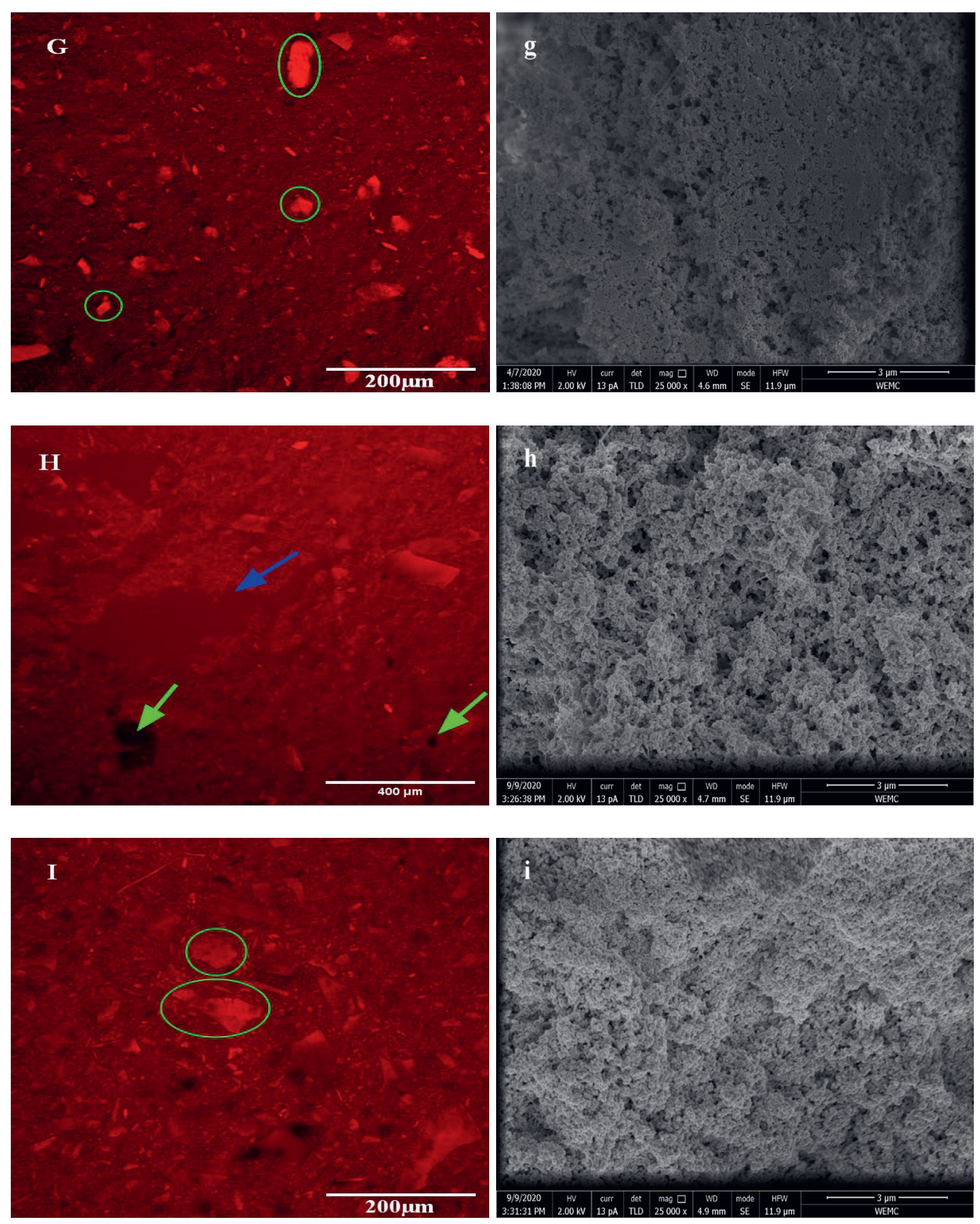


\subsection{Conclusions}

The present study provides in-depth and systematic information on the rheological properties of heat-set gels formed by native SPI and its hydrolysates, after selectively hydrolyzing $\beta$-conglycinin (DßH) or glycinin (DGH). SAOS tests showed that hydrolysates could form gels at earlier time and lower temperature than SPI, but the gels formed by SPI are more elastic. Hydrolyzing glycinin shortens the gelling points to a larger extent than hydrolyzing $\beta$-conglycinin, but the former hinders the stiffening process during heating since interactions (hydrophobic interaction and disulfide bonds) are weakened and more nonnetwork peptides are generated. On the other hand, DBH with a lower DH (DBH-L/UD) could form comparable or even stiffer gels than SPI, especially after dialysis (DBH-L/D). Scaling theories indicated that $\mathrm{D} \beta \mathrm{H}$ gels are weak-link type gels with higher $D_{f}$, while DGH gels are strong-link type gels with lower $D_{f}$. SPI gels are transition type gels with a $D_{f}$ in between. By using LAOS tests, the NLVE properties of these gels were also determined. SPI gels had a higher $\gamma_{c}$ than hydrolysates gels. With increasing strain, D $B H$ gels, DGH gels and SPI gels displayed strain softening (Type I), weak strain overshoot (Type III) and strong strain overshoot (Type IV) behaviors, respectively. Lissajous plots illustrated the transition of all gels from elastic dominant to plastic dominant. This decay of elasticity occurred at lower strains for DGH gels. In the medium strain range, SPI gels showed strong intracycle shear stiffening and $\mathrm{D} \beta \mathrm{H}$ gels showed strong intracycle shear thinning. In the large strain regime, SPI and D $\beta H$ gels showed abrupt yielding behavior while DGH gels yielded more gradually. The microstructure of gels well explained their different rheological properties. SPI and DBH gels have relatively denser networks which are built by large and compact flocs, while DGH gels have a more coarse and heterogeneous structure, with constituent flocs which are smaller and more branched. Our results established the "rheological fingerprint" of soy protein heat-set gels and show that the gel properties can be tailored by selective proteolysis, which provides a useful pathway for designing products based on soy protein gels. 


\subsection{Supplementary materials}

Figure S4.1. Heat-induced gelation profiles of the hydrolysates with low DH and the hydrolysates after dialysis. The temperature profile is indicated with a dashed line.
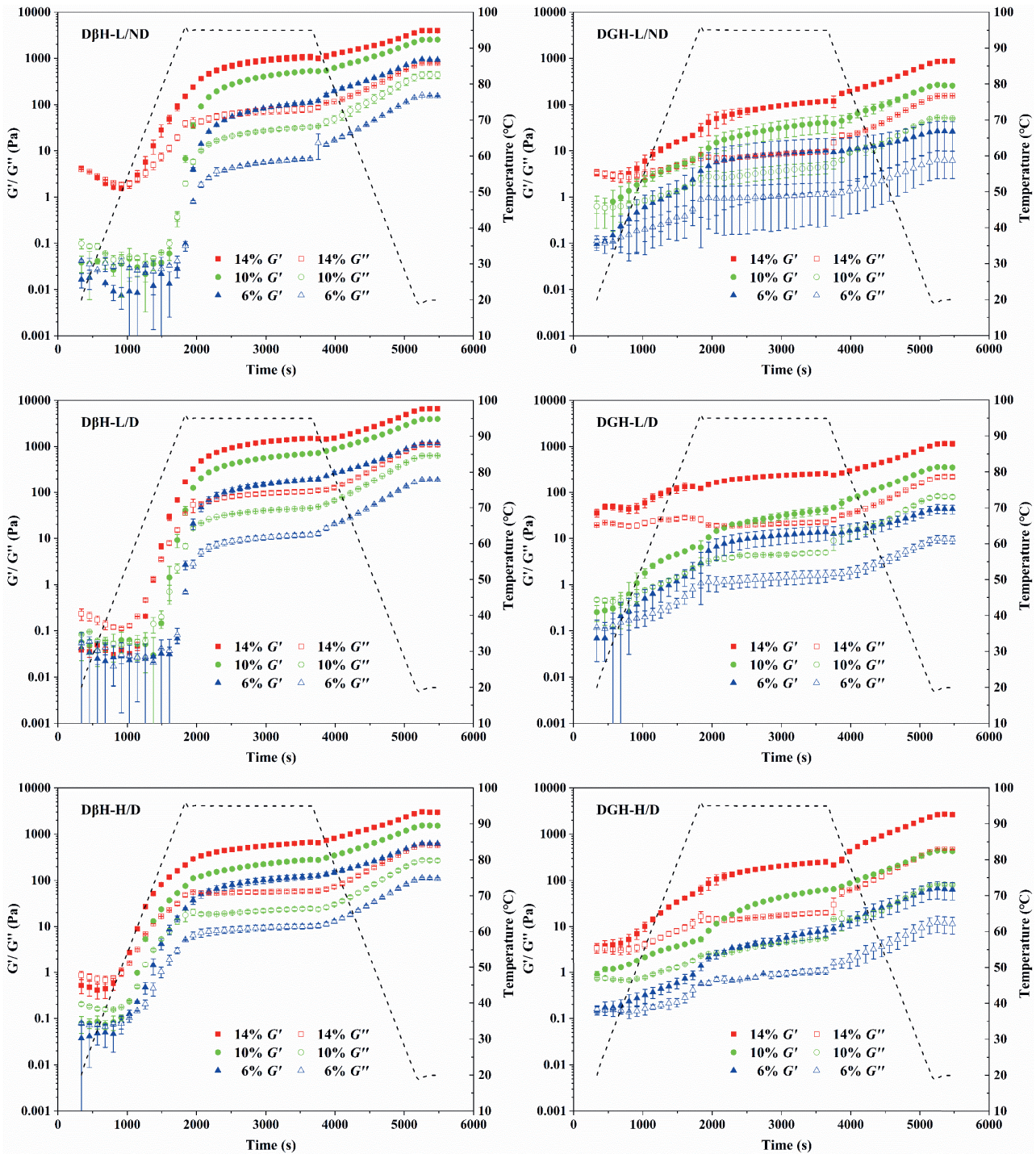
Figure S4.2. Strain amplitude sweep tests (from $1 \%$ to $1000 \%$, ramp logarithmic mode, at $1 \mathrm{~Hz}$ and $20{ }^{\circ} \mathrm{C}$.) on the heat-set gels formed by the hydrolysates with low DH and the hydrolysates after dialysis.
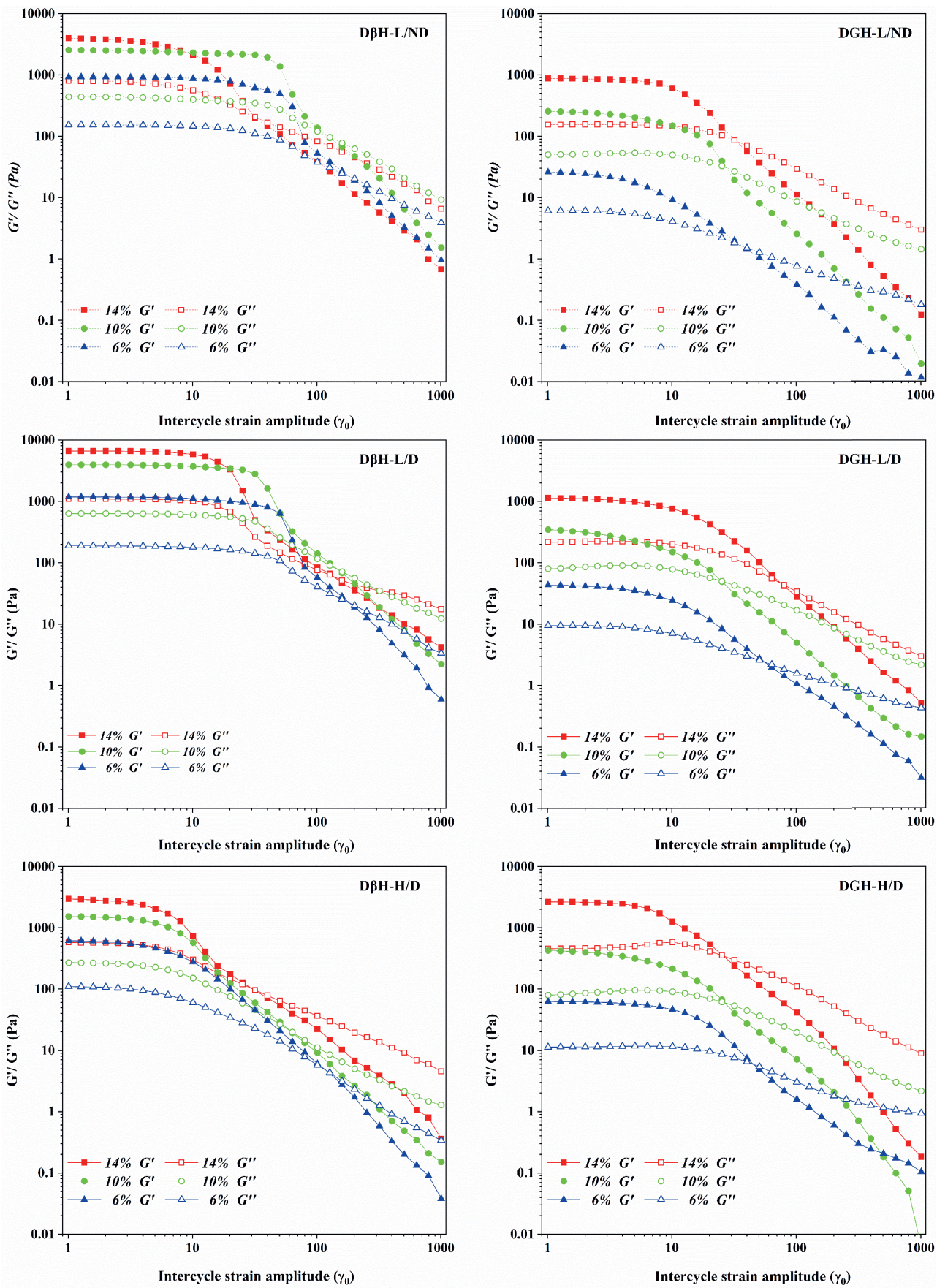
Figure S4.3. Elastic modulus at the largest strain $\left(G_{L}^{\prime}\right)$ and minimum strain $\left(G_{M}^{\prime}\right)(a)$, viscosity at the maximum shear rate $\left(\eta_{L}^{\prime}\right)$ and minimum shear rate $\left(\eta_{M}^{\prime}\right)(b)$ for the heat-set gels formed by $10 \%(w / w)$ of (from top to bottom) SPI, D $\beta H-L / N D, D \beta H-L / D, D \beta H-H / N D, D \beta H-H / D, D G H-L / N D, D G H-L / D, D G H-H / N D$, and DGH-H/D during the intercycle strain amplitude $(y o)$ sweep from $1 \%$ to $1000 \%$.
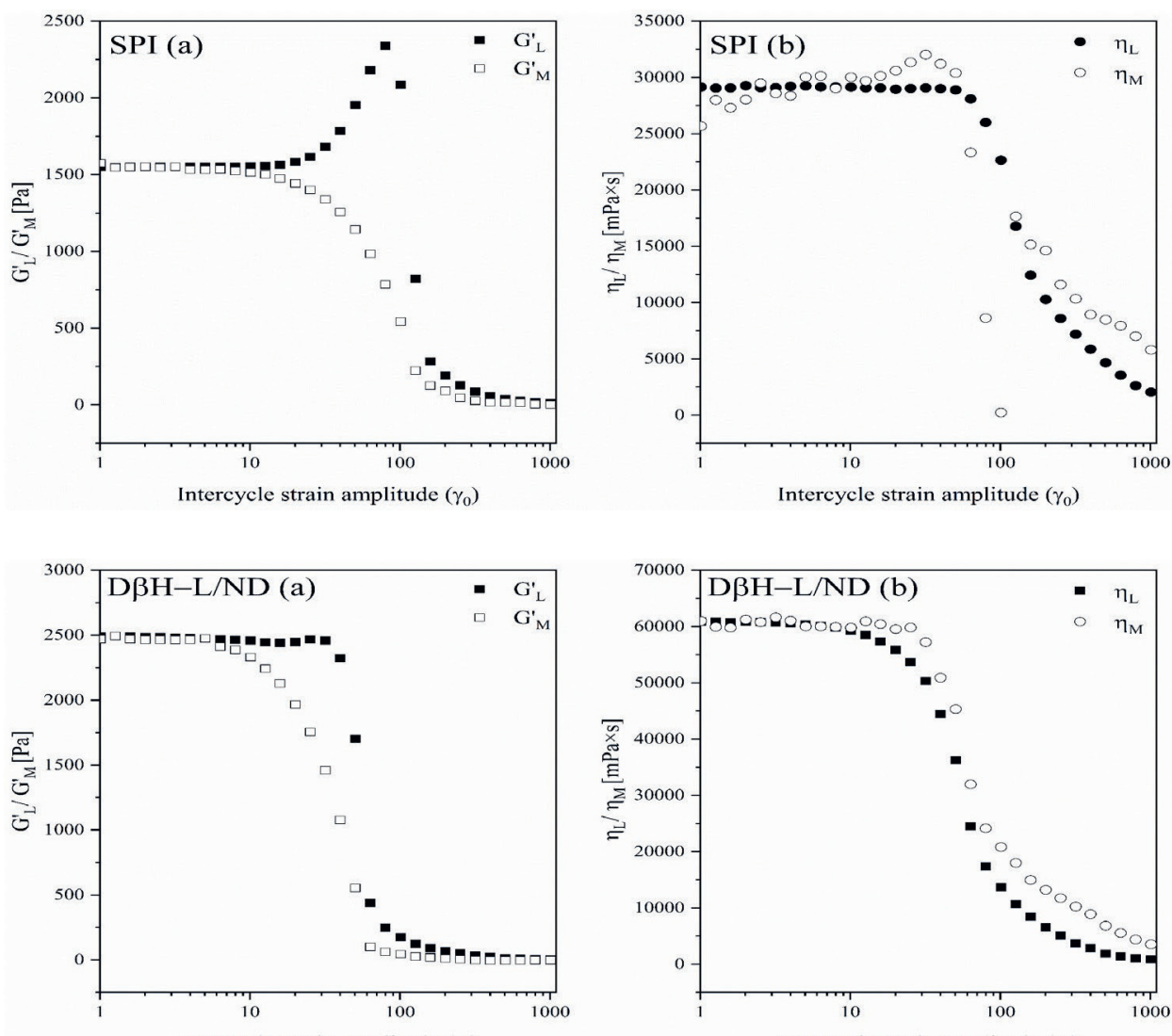

Intercycle strain amplitude $\left(\gamma_{0}\right)$

Intercycle strain amplitude $\left(\gamma_{0}\right)$
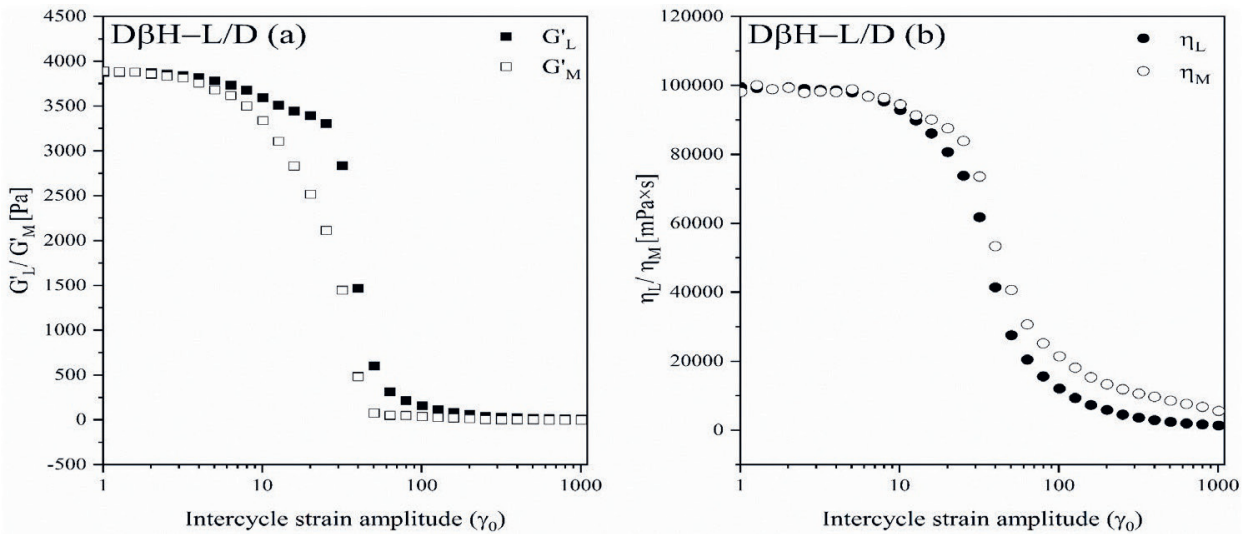

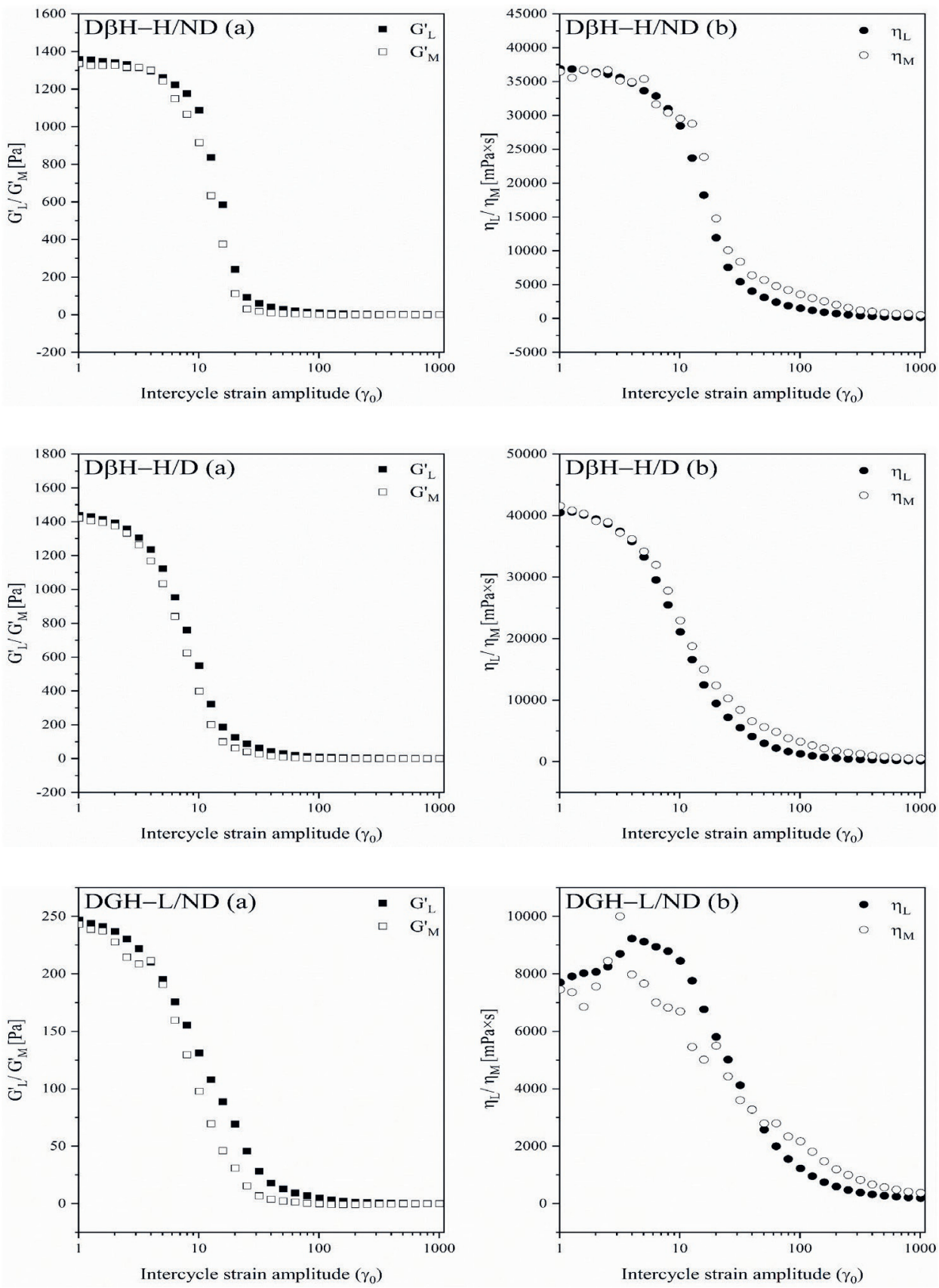

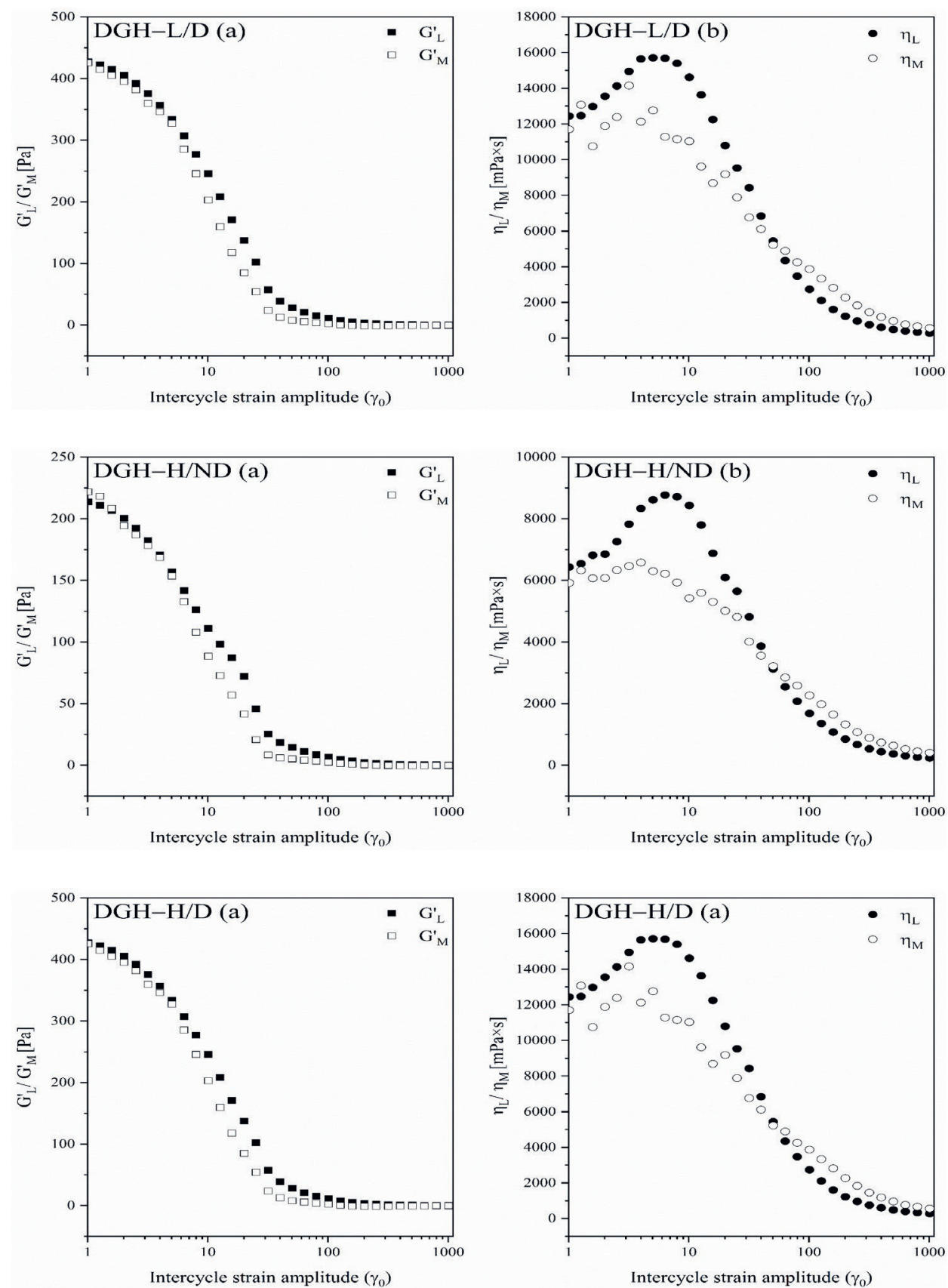
Table S4.1 Loss tangent (tan $\delta$ ) of SPI, D $\beta H$ and DGH heat-set gels at $1 \mathrm{~Hz}$ and $20^{\circ} \mathrm{C}$. Values are means \pm standard deviation. Different lowercase letters $(a, b, c \ldots)$ indicate significant differences $(p<0.05$, Duncan's test) in the same row. Different uppercase letters $(A, B, C \ldots)$ indicate significant differences $(p<0.05$, Duncan's test) in the same column.

\begin{tabular}{|c|c|c|c|}
\hline & $6 \%$ & $10 \%$ & $14 \%$ \\
\hline & $\tan \delta$ & $\tan \delta$ & $\tan \delta$ \\
\hline SPI & $0.122 \pm 0.001^{a, A}$ & $0.120 \pm 0.001^{a, A}$ & $0.121 \pm 0.001^{a, A}$ \\
\hline $\mathrm{D} \beta \mathrm{H}-\mathrm{L} / \mathrm{ND}$ & $0.166 \pm 0.003$ a, B & $0.173 \pm 0.025 a b, B C$ & $0.201 \pm 0.001$ b, D \\
\hline $\mathrm{D} \beta \mathrm{H}-\mathrm{L} / \mathrm{D}$ & $0.160 \pm 0.003^{a, B}$ & $0.161 \pm 0.002^{a, B}$ & $0.166 \pm 0.002$ b, B \\
\hline $\mathrm{D} \beta \mathrm{H}-\mathrm{H} / \mathrm{ND}$ & $0.175 \pm 0.000 a, c$ & $0.175 \pm 0.004^{a, B C}$ & $0.194 \pm 0.004$ b, D \\
\hline $\mathrm{D} \beta \mathrm{H}-\mathrm{H} / \mathrm{D}$ & $0.179 \pm 0.002 \mathrm{a}, \mathrm{c}$ & $0.177 \pm 0.001^{a, B C}$ & $0.196 \pm 0.004$ b, D \\
\hline DGH-L/ND & $0.237 \pm 0.010 \mathrm{c,E}$ & $0.196 \pm 0.004$ b, DE & $0.178 \pm 0.007 a, c$ \\
\hline DGH-L/D & $0.221 \pm 0.005$ b, D & $0.203 \pm 0.008^{a, E}$ & $0.193 \pm 0.006 a, D$ \\
\hline DGH-H/ND & $0.228 \pm 0.004^{c, D}$ & $0.183 \pm 0.004$ b, CD & $0.167 \pm 0.008^{a, B}$ \\
\hline DGH-H/D & $0.182 \pm 0.004^{b c, c}$ & $0.188 \pm 0.007^{c, C D E}$ & $0.173 \pm 0.004^{a, B C}$ \\
\hline
\end{tabular}




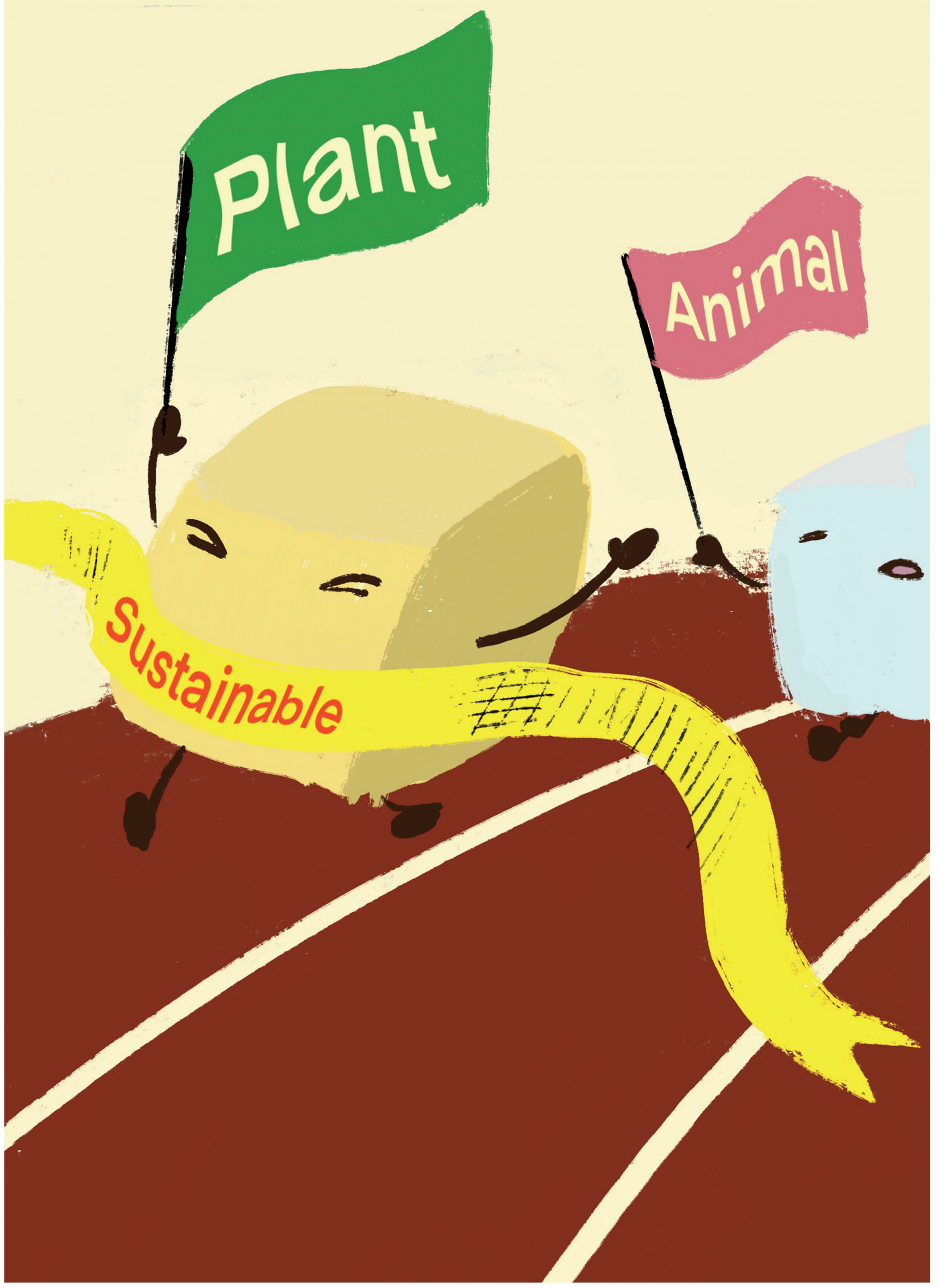




\section{Chapter 5}

\section{Acid-induced gels from soy and whey protein thermally-induced mixed aggregates: Rheology and microstructure}

To be submitted as:

Wenjie Xia, Linfeng Zhu, Roy J.B.M. Delahaije, Zhe Cheng, Xilong Zhou, Leonard M.C. Sagis. Acid-induced gels from soy and whey protein thermally-induced mixed aggregates: Rheology and microstructure. 


\section{Abstract}

In this study, we explored how substituting whey protein isolate (WPI) with soy protein isolate (SPI) affects the linear and non-linear rheological behavior of acid-induced gels, and their microstructures. Commercial SPI and WPI dispersions $(\mathrm{pH} 7.0,3.0 \mathrm{mS} / \mathrm{cm})$ were preheated $\left(95{ }^{\circ} \mathrm{C}, 30 \mathrm{~min}\right)$ at different protein concentrations $(2 \%, 4 \%, 6 \%$, and $8 \% \mathrm{w} / \mathrm{w})$ and SPI: WPI ratios (0:4, 1:3, 2: 2, 3: 1 and 4:0), and the resultant thermally-induced aggregates were characterized, before gelation was induced by glucono- $\delta$-lactone (GDL). Small and large amplitude oscillatory shear (SAOS and LAOS) tests showed that replacing WPI with SPI decreased the strength (lower $\mathrm{G}^{\prime}$ ) and stretchability (lower $\gamma_{c}$ ) of acid-induced gels in the linear viscoelastic regime. Gels containing SPI behaved more similar to pure SPI gels in the non-linear viscoelastic regime: displaying a relatively elastic response at large strain and a gradual transition to plastic behavior. The changes in rheological properties were explained by the differences in the gel microstructures, via fractal scaling theory, multiphoton laser scanning microscopy (MLSM) and scanning electron microscopy (SEM). WPI gels formed denser and homogenous gel networks with very strong inter-floc links, while the hybrid gels and pure SPI gels formed coarser and more porous networks with intermediate inter-floc links. The constituent flocs in the latter were larger with rougher, more elongated and branched structures. The present results provide useful information for future attempts to replace WPI with SPI in food products based on acid-induced gelation. 


\subsection{Introduction}

The demand for food protein has grown dramatically due to the global population increase. This demand cannot be met by simply increasing the supply of traditional animal-based proteins such as meat and dairy protein, considering their high ecological footprint and production cost compared to plant-based proteins [173]. Dairy proteins, i.e. caseins and whey proteins, have been widely used as gelling agents in texturized foods such as cheese, yoghurt, and pudding. If they can be partially or completely substituted by plant proteins in these gel-based products, the new formulations will not only be more sustainable, but also bring more choices and nutritional values to the consumer $[173,174]$.

Gelation of food proteins is a complex process that involves several reactions such as denaturation, dissociation-association, aggregation and gelation [175]. This process is usually induced by heat treatment, and the properties of heat-set gels have been extensively studied $[176,177]$. On the other hand, protein gelation can also be induced by acidulating agents, such as glucono- $\delta$-lactone (GDL). Acid-induced gelation occurs in the production of many food products, such as yoghourt and silken tofu. In contrast to heat-induced gelation where the denaturation, aggregation and gelation are intertwined and occur simultaneously, acid-induced gelation often consists of two separate steps: Firstly, protein solutions are preheated at neutral $\mathrm{pH}$ and a relatively low concentration to induce protein denaturation and aggregation. Secondly, acidulating agents are used to decrease the $\mathrm{pH}$ of the protein aggregate dispersions. This reduces the net charge of the proteins and hence the electrostatic repulsion between them, leading to further aggregation and gel formation $[178,179]$. Since the gelation step can be performed at ambient temperature, acid-induced gels are also known as cold-set gels. They can be used to encapsulate heat-sensitive or volatile compounds in the food, pharmaceutical and cosmetic sectors [180].

The properties of acid-induced gels can be influenced by the conditions of preheating and gelation processes (e.g. temperature, time, $\mathrm{pH}$, and ionic strength) as well as the concentration and composition of proteins [181-184]. For acid-induced hybrid gels formed by mixed dairy and plant proteins, the properties can be expected to vary significantly with composition, since proteins from different origins normally possess different structures, thermal transition temperatures, isoelectric points, and gelling properties [174]. For the preheating step these hetero-proteins can be heated separately or simultaneously, leading to "a mixture of aggregates" or "mixed aggregates", which would also result in different gel properties [185]. Up-to-now, studies have been reported on acid-induced hybrid gels formed by pea and whey proteins $[184,185]$, pea protein and casein micelles [186], soy protein and sodium caseinate [187], and soy protein and casein micelles [188]. Information on acid- 
induced gelation of whey and soy protein mixtures is still limited, although it is of both fundamental and applied interest.

The practical application of a gel system calls for a comprehensive understanding of its rheological properties. To characterize these properties, dynamic oscillatory shear tests are commonly performed, during which a gel material is subjected to a sinusoidal deformation, and the resulting mechanical response is measured [139]. Based on the amplitude of deformation, dynamic oscillatory shear tests can be divided into two categories: small amplitude oscillatory shear (SAOS) and large amplitude oscillatory shear (LAOS) tests. In SAOS tests, the strain amplitudes are too small to disrupt the gel microstructure, and the mechanical response of gels is in the linear viscoelastic (LVE) regime, i.e. the viscoelastic moduli ( $\mathbf{G}^{\prime}$ and $\mathbf{G}^{\prime \prime}$ ) are independent of the applied stress or strain [147]. In LAOS tests, on the other hand, the strain amplitude is increased to a level where the gel microstructure is affected, and the rheological properties in the non-linear viscoelastic (NLVE) regime can be revealed [176].

As mentioned in a recent review article by Joyner, despite LAOS tests are still far from the mainstream of the food science field, it is a valuable tool for investigating the interplay of food microstructures, textures, processing properties, and sensory properties, since food products inevitably undergo remarkably large deformation during production (e.g. mixing and pumping) and digestion processes (e.g. chewing and swallowing) [189]. The research group of Melito [140-143] has applied LAOS tests on whey protein isolate/k-carrageenan gels and different kinds of commercial cheeses, which demonstrated that the sensory and oral processing characteristics of food gels can be well correlated to their rheological properties in the NLVE regime. Bi et al. found the acid-induced hybrid gels of soy protein and locust bean gum/k-Carrageenan displayed intercycle strain softening behavior in the NLVE regime $[144,145]$. This behavior was also found when the fermentation-induced pea protein gels were subjected to LAOS tests, but these gels displayed intracycle strain stiffening behavior when the amplitude was between $1.6 \%$ and $160 \%$, and intracycle shear thinning behavior when the amplitude exceeded 160\% [134,149]. LAOS tests have also been applied on gelatin gels [190,191], waxy rice starch gels [147], pectin-Ca gels [192], and concentrated protein gels [150], to provide more comprehensive information on the linear and non-linear rheological behaviors of these food gel matrices.

Dairy proteins can be used to control the structure and texture of food products. Whey protein, for example, has been known to significantly affect the rheological properties of foods when processed under shear or different physicochemical environments [193]. Heating milk before acidification could cause denaturation and induce attractive interactions between whey proteins, which conferred higher elastic moduli $\left(G^{\prime}\right)$ to the milk gels [194]. 
Adding whey protein to milk before this thermal treatment also enhanced the gel firmness and reduced syneresis [195]. Soy protein, a plant-based protein that has been widely consumed in Asia, is also known for its contribution to food texture, as well as its high nutritional value and availability [124]. In our recent work, we applied both SAOS and LAOS tests on soy protein heat-set gels and characterized their rheological behavior in both the LVE and NLVE regimes [176]. Nevertheless, it remains unclear that how mixing soy protein with whey protein affects the "rheological fingerprint" of acid-induced gel matrices, especially in the NLVE regime.

In this study, we investigated the rheological properties and microstructure of acid (GDL)induced gels formed by blends of commercial whey protein isolate (WPI) and soy protein isolate (SPI). Before acidification, WPI and SPI were preheated together at different ratios and concentrations, and thus mixed aggregates were formed. The properties of these thermally-induced aggregates were characterized by dynamic light scattering (DLS), size exclusion chromatography (SEC), surface hydrophobicity $\left(\mathbf{H}_{\mathbf{0}}\right)$, and intrinsic fluorescence spectroscopy. Subsequently, these aggregates were used to form acid-induced gels, of which the linear and non-linear viscoelastic properties were studied by SAOS and LAOS tests, and the water holding capacity (WHC) and gel solubility were also determined. We applied Lissajous-Bowditch plots to interpret the LAOS data, and used fractal scaling theory to link the rheological behaviors to the gel microstructure (characterized by multiphoton laser scanning microscopy (MLSM) and scanning electron microscopy (SEM)). This study will benefit the application of soy protein in traditional dairy products based on acid-induced gelation.

\subsection{Materials and methods}

\subsubsection{Materials}

Commercial SPI (Unisol NRG IP Non-GMO) was provided by Barentz International B.V. (Hoofddorp, North Holland, the Netherlands). Commercial WPI (BiPRO ${ }^{\circledR} 9500$ ) was bought from AGROPUR Dairy Co. (Longueuil, Quebec, Canada). The protein content of SPI and WPI were $86.57 \pm 0.18 \%(N \times 6.25)$ and $92.26 \pm 0.29 \%(N \times 6.38)$ respectively, determined by Dumas combustion method with a FlashEA 1112 N/Protein Analyzer (Thermo Fisher Scientific, Waltham, MA, USA). Glucono- $\delta$-lactone (GDL) (Art. No. G4750) and other chemical reagents were ordered from Sigma-Aldrich Co., (St. Louis, MO, USA). Deionized distilled water (DDW) used in the study was purified by a PURELAB Ultra apparatus (ELGA LabWater Co., High Wycombe, UK). Unless mentioned otherwise, all experiments were performed in thermostatic laboratories $\left(20 \pm 1^{\circ} \mathrm{C}\right)$. 


\subsubsection{Sol-gel phase diagram}

Commercial SPI and WPI powders were dissolved in DDW at different protein concentrations and stirred overnight to ensure complete hydration. SPI and WPI dispersions of the same concentration were mixed at different ratios and heated simultaneously at $95^{\circ} \mathrm{C}, \mathrm{pH} 7.0$ for 30 min. Subsequently, a sol-gel phase diagram was established for these heated protein samples according to the method of Chihi et al. [185]. To determine the critical thermal gelation concentrations $\left(\boldsymbol{C}_{\boldsymbol{g t h}}\right)$, the samples after heating $(5 \mathrm{~mL})$ were immediately poured into $15 \mathrm{ml} \mathrm{CELLSTAR}{ }^{\circledR}$ tubes with screw caps (Art. No. 188271, Greiner Bio-one B.V., the Netherlands) and vertically stored at $4{ }^{\circ} \mathrm{C}$ for $24 \mathrm{~h}$. After this, the samples that were not flowing under tube inversion were considered as heat-set gels, and the minimum concentration to form these gels was considered as $C_{g t h}$. To determine the critical acid gelation concentration $\left(\boldsymbol{C}_{\boldsymbol{g a}}\right)$, samples $(5 \mathrm{ml})$ with protein concentrations below $\boldsymbol{C}_{g \text { th }}$ were transferred into $15 \mathrm{ml} \mathrm{CELLSTAR}{ }^{\circledR}$ tubes after heating. When the samples were cooled to room temperature, appropriate amounts of GDL powder ( $20 \%$ of total protein content) were added [196]. The resulting samples were incubated at $40{ }^{\circ} \mathrm{C}$ for $3 \mathrm{~h}$ and then stored at $4{ }^{\circ} \mathrm{C}$ for $24 \mathrm{~h}$. Those not flowing under tube inversion were considered as acid-induced gels, and the minimum concentration to form gels was considered as $C_{g a}$.

\subsubsection{Characterization of SPI and WPI thermally-induced aggregates}

The effects of SPI: WPI ratio on the properties of thermally-induced aggregates were studied using samples with a $4 \% \mathrm{w} / \mathrm{w}$ protein concentration. SPI and WPI dispersions $(4 \% \mathrm{w} / \mathrm{w})$ were prepared and mixed at five ratios $(4: 0,3: 1,2: 2,1: 3,0: 4)$. The $\mathrm{pH}$ and conductivity of these dispersions were standardized to $\mathrm{pH} 7.0$ and $3.0 \mathrm{mS} / \mathrm{cm}$ ( $\sim 55 \mathrm{mM}$ ionic strength). Subsequently, each dispersion was divided into two parts for the following tests. One part was heated at $95^{\circ} \mathrm{C}, \mathrm{pH} 7.0$ for 30 min while the other one was not heated and served as a control.

\section{Dynamic light scattering (DLS)}

The particle size and zeta-potential of samples were measured by DLS using a Zetasizer Nano ZS (Malvern Instruments, Worcestershire, UK). Sample dispersions were diluted to $0.3 \% \mathrm{w} / \mathrm{w}$ protein concentration and injected into folded capillary zeta cells (DTS1070, Malvern). The refractive and absorption indices of the particles were set at 1.450 and 0.001 respectively, while the refractive index of the dispersant (DDW) was 1.330 . 


\section{Size exclusion chromatography (SEC)}

Sample dispersions were centrifuged at $15000 \mathrm{~g}$ for $10 \mathrm{~min}$ before SEC analysis. The supernatants $(50 \mu \mathrm{L})$ were injected on an ÄKTA pure 25 system (Cytiva, Marlborough, MA, USA) equipped with Superose ${ }^{\circledR} 610 / 300 \mathrm{GL}$ column, and eluted with sodium phosphate buffer ( $30 \mathrm{mM}, \mathrm{pH} 7.0$ ) containing $50 \mathrm{mM} \mathrm{NaCl}$ at a flow rate of $0.5 \mathrm{~mL} / \mathrm{min}$. The elution was monitored using UV absorbance at $214 \mathrm{~nm}$. The column was calibrated with a series of biomacromolecules with molecular weights ranging from 29 to 2000 kDa (MWGF-1000, Sigma-Aldrich, USA). The calibration curve of these standard samples is provided in the supplementary material (Fig. S5.1).

\section{Surface hydrophobicity (HO)}

The $\mathrm{H}_{0}$ of samples was determined using 8-anilino-1-napthalenesulfonic acid ammonium salt (ANSA) as a fluorescent probe. Each sample dispersion was diluted with DDW to obtain a sequence of protein concentrations $(0.0025 \%, 0.005 \%, 0.01 \%, 0.02 \% \mathrm{w} / \mathrm{w})$, and were added into acrylic cuvettes (Sarstedt Inc, Nümbrecht, Germany) together with $40 \mu \mathrm{L}$ of ANSA reagent $(8 \mathrm{mM})$. After one-hour reaction in the dark, the fluorescence intensity (FI) of these samples was measured by a luminescence spectrometer LS50B (Perkin Elmer, Waltham, MA, USA) at an excitation wavelength ( $\left.\boldsymbol{\lambda}_{\mathrm{Ex}}\right)$ of $390 \mathrm{~nm}$ and emission wavelength $\left(\boldsymbol{\lambda}_{\mathrm{Em}}\right)$ of $470 \mathrm{~nm}$. The slope of the plot of FI vs. protein concentration was used as the indicator of $\mathrm{H}_{0}$.

\section{Intrinsic fluorescence spectra}

Intrinsic fluorescence spectra of samples were measured according to the method of Wan et al. [23]. The sample dispersions were adjusted to $0.02 \% \mathrm{w} / \mathrm{w}$ and their intrinsic fluorescence spectra were determined using a Perkin Elmer (LS50B) luminescence spectrometer. A volume of $3 \mathrm{~mL}$ of each sample was slowly added into a $10 \mathrm{~mm}$ path length quartz cuvette (Hellma $\mathrm{GmbH}$ ). The $\lambda_{\mathrm{Ex}}$ was set at $295 \mathrm{~nm}$, and the $\lambda_{\mathrm{Em}}$ was recorded from 300 to $500 \mathrm{~nm}$. Both the excitation and emission slit were $5 \mathrm{~nm}$.

\section{Protein solubility}

The protein solubility of samples was evaluated by measuring the protein content in the supernatant after centrifugation. Sample dispersions $(20 \mathrm{ml}$ ) were added in $50 \mathrm{ml}$ centrifuge tubes (Sarstedt Inc) and then centrifuged at $15,000 \mathrm{~g}$ for $10 \mathrm{~min}$. The protein concentration of supernatants was measured by the Dumas combustion method. Protein solubility of different samples was expressed as below: 
Protein solubility $(\%)=\frac{\text { Protein content supernatant }}{\text { Total protein content }} \times 100 \%$

\subsubsection{Rheology of acid-induced gelation}

The process of acid-induced gelation and the rheological properties of the resultant gels were studied using an MCR 302 rheometer (Anton Paar, Graz, Austria) with a titanium sandblasted concentric cylinder geometry (CC17). SPI and WPI dispersions at different protein concentrations $(2 \%, 4 \%, 6 \%$ and $8 \% \mathrm{w} / \mathrm{w})$ were prepared, and then mixed at different ratios $(4: 0,3: 1,2: 2,1: 3,0: 4)$. Each protein mixture was adjusted to $\mathrm{pH} 7.0$ and $3.0 \mathrm{mS} / \mathrm{cm}$ ( $\sim 55 \mathrm{mM}$ ionic strength) before being heated at $95^{\circ} \mathrm{C}, \mathrm{pH} 7.0$ for $30 \mathrm{~min}$. After this, the preheated samples were cooled back to room temperature and mixed with appropriate amounts of GDL ( $20 \%$ of total protein content). Immediately, a volume of 4.7 $\mathrm{ml}$ protein sample was gently injected into the CC17 cup that was preheated at $40{ }^{\circ} \mathrm{C}$. The CC17 cup was covered with a solvent trap to prevent sample evaporation. In the following rheological tests, the temperature was controlled by a Peltier element and a water circulation system.

\section{Small amplitude oscillatory shear (SAOS) tests}

SAOS tests were performed at a fixed amplitude of $0.1 \%$ and a frequency of $0.1 \mathrm{~Hz}$, which were within the linear viscoelastic (LVE) regime. First, oscillations were applied at $40^{\circ} \mathrm{C}$ for $3 \mathrm{~h}$, and then the temperature was decreased from $40{ }^{\circ} \mathrm{C}$ to $4^{\circ} \mathrm{C}$ at a rate of $1^{\circ} \mathrm{C} / \mathrm{min}$. After this, the formed gels were allowed to rest at $4^{\circ} \mathrm{C}$ for $5 \mathrm{~min}$, before performing a frequency-sweep test from 0.1 to $10 \mathrm{~Hz}\left(4^{\circ} \mathrm{C}\right)$, at an amplitude of $0.1 \%$. The storage modulus $\left(\mathbf{G}^{\prime}\right)$, loss modulus ( $\left.\mathbf{G}^{\prime \prime}\right)$, and loss tangent (tan $\boldsymbol{\delta}$ ) were recorded throughout the SAOS tests. The change of $\mathrm{G}^{\prime}$ and $\mathrm{G}^{\prime \prime}$ as a function of frequency was fitted with a Power-law model.

\section{Large amplitude oscillatory shear (LAOS) tests}

After the frequency-sweep test in Section 2.4.1, the gels were subjected to a LAOS amplitude-sweep test to investigate their viscoelastic behavior in the non-linear viscoelastic (NLVE) regime. The strain amplitude ( $\boldsymbol{Y o}$ ) was increased from $0.1 \%$ to $1000 \%$ in a ramp logarithmic mode at a fixed frequency $(0.1 \mathrm{~Hz})$ and temperature $\left(4^{\circ} \mathrm{C}\right)$. The $\mathrm{G}^{\prime}$ and $\mathrm{G}^{\prime \prime}$ were monitored as a function of intercycle strain $(\boldsymbol{\gamma} \boldsymbol{o})$ while the shear stress $(\boldsymbol{\sigma})$ was monitored as a function of intracycle strain $(\boldsymbol{y})$ and strain rate $(\dot{\gamma})$. Lissajous-Bowditch plots (also called Lissajous plots) were constructed to analyze the NLVE properties of these gels. As extensively discussed by Ewoldt et al. [25], the analysis framework using Lissajous plots can effectively reveal the different non-linear rheological responses of materials. 


\subsubsection{Acidification kinetics}

The effects of SPI: WPI ratio on the acidification kinetic during GDL-induced gelation were studied using samples with a $4 \% \mathrm{w} / \mathrm{w}$ protein concentration. In brief, SPI and WPI dispersions were prepared and mixed at five ratios $(4: 0,3: 1,2: 2,1: 3,0: 4)$ as in Section 5.2.4. Then, the dispersions were heated at $95{ }^{\circ} \mathrm{C}, \mathrm{pH} 7.0$ for $30 \mathrm{~min}$ and cooled to room temperature. After mixing with GDL powder $(0.8 \% \mathrm{w} / \mathrm{w})$, the dispersions were incubated in a water bath at $40{ }^{\circ} \mathrm{C}$ for $3 \mathrm{~h}$, during which their $\mathrm{pH}$ were recorded every $10 \mathrm{~min}$ by a $\mathrm{pH}-$ meter (SCHOTT Instruments, Mainz, Germany).

\subsubsection{Water holding capacity (WHC)}

The WHC of acid-induced gels was determined based on the method of Kocher and Foegeding [197]. A microcentrifuge filtration unit (i.e. a $2 \mathrm{ml}$ Eppendorf tube with a spin tube inside) was used (Axygen Biosciences, Inc., Union City, USA). Filter paper was used to reduce grid size. Protein gels $(4 \% \mathrm{w} / \mathrm{w}, 0.5 \mathrm{~mL})$ were prepared in the same manner as in the rheology test (Section 5.2.4) and centrifuged at $500 \mathrm{~g}$ for $10 \mathrm{~min}$ ( $Z 306$ centrifuge, HERMLE Labortechnik, Wehingen, Germany). The water expelled from the gel was collected in the outer Eppendorf tube. The WHC was defined as the percentage of the water that remaining in the gel after centrifugation:

$$
W H C=\left(W_{T}-W_{E}\right) / W_{T} \times 100[\%]
$$

where $W_{T}$ is the total amount of water before gelation $(g)$, and $W_{E}$ is the amount of water expelled from the gel $(\mathrm{g})$.

\subsubsection{Gel solubility}

The solubility of acid-induced gels was determined according to previous studies $[198,199]$. Protein gels $(4 \% \mathrm{w} / \mathrm{w}, 10 \mathrm{ml})$ were formed in $50 \mathrm{ml}$ centrifuge tubes (Sarstedt Inc) before mixing with $10 \mathrm{ml}$ of each of the following solvents: $\left(\mathbf{S}_{\mathbf{A}}\right)$ DDW; $\left(\mathbf{S}_{\mathbf{B}}\right) 0.086 \mathrm{M}$ Tris, $0.09 \mathrm{M}$

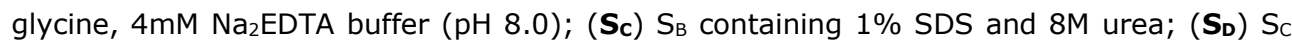
containing $5 \% 2$-mercaptoethanol. After this, the tubes were rotated by a rotator (Labinco $\mathrm{BV}, \mathrm{Breda}$, The Netherlands) for 48 hours and then centrifuged at $15000 \mathrm{~g}$ for $10 \mathrm{~min}$ (Z383K centrifuge, HERMLE Labortechnik $\mathrm{GmbH}$, Wehingen, Germany). Soluble protein content in the supernatant was determined using a Pierce ${ }^{\mathrm{TM}}$ detergent compatible Bradford assay kit (No. 23246, Thermo Fisher Scientific). The gel solubility in different solvents was expressed as below: 


$$
\text { Gel solubility }(\%)=\frac{\text { Protein } \text { content supernatant }}{\text { Total protein content }} \times 100 \%
$$

Tris-glycine buffers can screen electrostatic interactions, SDS and urea are known to disrupt hydrophobic interactions and hydrogen bonds, and 2-mercaptoethanol is a bond-breaking agent for disulfide bonds. The differences in gel solubility in different solvents (i.e. $S_{B}-S_{A}$, $\mathrm{S}_{C}-\mathrm{S}_{B}$, and $\mathrm{S}_{D}-\mathrm{S}_{\mathrm{C}}$ ) were used to indicate the relative importance of different interactions for maintaining the gel structures.

\subsubsection{Microscopy of the acid-induced gels}

\section{Multiphoton laser scanning microscopy (MLSM)}

Alexa Fluor ${ }^{\circledR} 350$ ( $\left.\lambda_{E x}: 800 \mathrm{~nm}, \lambda_{\mathrm{Em}}: 570-630 \mathrm{~nm}\right)$ and Cy $5^{\circledR}\left(\lambda_{\mathrm{Ex}}: 840 \mathrm{~nm}, \lambda_{\mathrm{Em}}: 650-700 \mathrm{~nm}\right)$ were dissolved in dimethyl sulfoxide at $1 \mathrm{mg} / \mathrm{mL}$, and then used to stain WPI and SPI dispersion $(4 \% \mathrm{w} / \mathrm{w})$, respectively. Then, these two dispersions were mixed at different ratios before mixing with GDL $(0.8 \% \mathrm{w} / \mathrm{w})$. Immediately, $120 \mu \mathrm{L}$ of each mixture was transferred to hermetically sealed flat cuvettes (Gene Frame ${ }^{\circledR} 125 \mu \mathrm{L}$, Thermo Fisher Scientific, Waltham, MA, USA) that were glued onto microscopy slides in advance. After covering with coverslips, these microscopy slides were mounted on a Peltier system (LTS120, LINKAM. Co., UK) to allow acid-induced gels to form in the same manner as in the rheology tests $\left(40{ }^{\circ} \mathrm{C}\right.$ for 3 hours and cooled to $4{ }^{\circ} \mathrm{C}$ at the rate of $1{ }^{\circ} \mathrm{C} / \mathrm{min}$ ). The gel samples were visualized by a Leica SP8Dive multiphoton excitation microscope (Leica, Germany), using a HC FLUOTAR L 25×/0.95 W VISIR objective.

\section{Scanning electron microscopy (SEM)}

Acid-induced gels $(4 \% \mathrm{w} / \mathrm{w}, 5 \mathrm{~mL})$ were prepared in $10 \mathrm{ml}$ disposable thermotolerant syringes (Terumo Corporation, Tokyo, Japan) with the tip sealed by a syringe screw cap (Combi $^{\text {tm }}$ plug, Becton Dickinson, Franklin Lakes, NJ, USA). For SEM imaging, the gels formed in the syringes were cut into small cubes $(\sim 5 \times 5 \times 5 \mathrm{~mm})$ and fixed in $2.5 \%$ glutaraldehyde for at least 8 hours. After the crosslinking, the samples were rinsed to remove the excess of glutaraldehyde and were dehydrated using a series of ethanol $(30 \%, 50 \%, 70 \%, 80 \%, 90 \%$, $100 \%$ ) solutions before critical point drying (CPD 300, Leica, Vienna, Austria). Subsequently, the samples were fractured and attached to SEM sample holders using Carbon Conductive Cement (Leit-C, Neubauer Chemicalien, Germany). After sputter coating with a $12 \mathrm{~nm}$ layer of tungsten (SCD 500, Leica, Vienna, Austria) the fractured surfaces were analyzed with SE detection at $2 \mathrm{kV}$ in a field emission scanning electron microscope (Magellan 400, FEI, Eindhoven, the Netherlands). 


\subsubsection{Statistical analysis}

All experiments were performed in duplicate at least. The statistical analysis of data was conducted by SPSS 25.0 (IBM SPSS Inc. Chicago, IL, USA). One-way ANOVA (one-way analysis of variance) with the Duncan post-Hoc test $(p<0.05)$ were used to evaluate the statistical significance of differences among means. Lissajous plots were made by MATLAB R2018b (MathWorks Inc., MA, USA) while other figures were made by Origin 2018 (Origin Lab Corporation, MA, USA).

\subsection{Results and discussion}

\subsubsection{Sol-gel phase diagram}

To investigate acid-induced gelation, it is necessary to avoid forming heat-set gels directly after the preheating step $\left(95^{\circ} \mathrm{C}, \mathrm{pH} 7.0\right.$ for $30 \mathrm{~min}$ ). A sol-gel phase diagram (Fig. 5.1) was established for SPI and WPI mixtures, using a series of samples covering different protein concentrations and SPI: WPI ratios. The red line in the phase diagram represents the critical thermal gelation concentration $\left(\boldsymbol{C}_{\boldsymbol{g t h}}\right)$, while the blue line represents the critical acid gelation concentration $\left(\boldsymbol{C}_{\boldsymbol{g a}}\right)$. As shown in Fig. 5.1, pure SPI and WPI directly formed heat-set gels when their concentration is above $8 \% \mathrm{w} / \mathrm{w}$ and $10 \% \mathrm{w} / \mathrm{w}$ respectively, which are comparable to the $C_{g t h}$ found in previous studies $[21,200]$. For SPI and WPI mixtures, the $C_{g t h}$ gradually decreased from $10 \%$ to $7.5 \% \mathrm{w} / \mathrm{w}$ with increasing SPI:WPI ratio, indicating the thermal gelling ability of mixtures increased as more soy protein was involved. The $C_{g a}$ for preheated SPI and WPI were $1.5 \%$ and $1.0 \% \mathrm{w} / \mathrm{w}$, respectively, and the $C_{g a}$ for the preheated mixtures are in between. This shows that after the preheating step, GDL-induced gels can be formed by commercial SPI and WPI at very low concentrations compared to heat-set gels. Based on this phase diagram, four protein concentrations ( $2 \% \mathrm{w} / \mathrm{w}, 4 \% \mathrm{w} / \mathrm{w}, 6 \% \mathrm{w} / \mathrm{w}$, and $8 \% \mathrm{w} / \mathrm{w})$, and five SPI: WPI ratios $(0: 4,1: 3,2: 2,3: 1$, and 4:0) were selected, to investigate acidinduced gelation of SPI and WPI mixtures. 


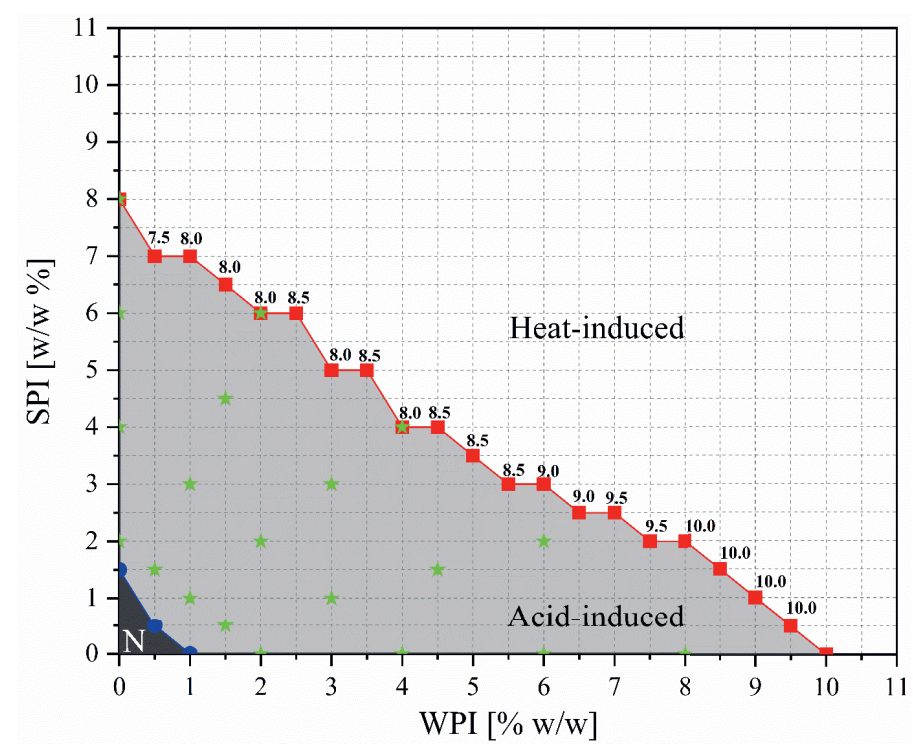

Figure 5.1 Sol-gel phase diagram for SPI and WPI mixtures.

\subsubsection{Characteristics of thermally-induced aggregates}

In the preheating step of this study $\left(95^{\circ} \mathrm{C}, \mathrm{pH} 7.0\right.$ for $30 \mathrm{~min}$ ), the mixtures of SPI and WPI formed thermally-induced aggregates, and we will first present an overview of the physical and conformational properties of these mixed aggregates before discussing the rheological and microstructural properties of the acid-induced gels formed by them.

Fig. 5.2A shows that the particle size distribution (PSD) (number based) of all samples moved towards larger sizes after preheating, proving the formation of protein aggregates. For instance, the peak in the PSD of pure WPI shifted from $\sim 2 \mathrm{~nm}$ to $\sim 20 \mathrm{~nm}$ after heating, which is comparable to results reported previously [201]. Both before and after the preheating, the PSD of mixtures shifted towards a larger size range when the proportion of SPI increased. Pure WPI and mixtures formed thermally-induced aggregates with a size between 10-100 nm, while pure SPI showed the existence of some large macroaggregates $(>100 \mathrm{~nm})$. The PSD result was obtained based on the whole protein sample, and we further applied size exclusion chromatography (SEC) to provide information on the soluble proteins and protein aggregates in the sample. As shown in Fig. 5.2B, the supernatants of unheated samples all displayed a unimodal SEC profile with one peak located in between 17.8 to 19.3 $\mathrm{mL}$ elution volume ( $\approx 18$ to $43 \mathrm{kDa}$ ), which can be attributed to protein monomers and subunits $[33,202]$. After preheating, the SEC profiles of all supernatants changed to a bimodal distribution, with a new peak appearing at around $7.6 \mathrm{~mL}$ elution volume $\left(\approx 17 \times 10^{3}\right.$ $\mathrm{kDa})$, representing the formation of soluble protein aggregates. As the SPI: WPI ratio 
increased, the location of this new peak did not change while its intensity decreased significantly, suggesting the size of soluble aggregates were similar but their amount decreased with increasing SPI proportion. Combined with the PSD results, it can be deduced that replacing WPI with SPI led to an increased formation of insoluble aggregates and a decrease in the fraction of smaller soluble aggregates. This analysis is also supported by protein solubility results (Fig. 5.2C) which displayed a linear relationship with the enclosed area of SEC peaks (Fig. S5.2). The preheating step increased the protein solubility of all samples significantly. After heating, the solubility showed a decreasing trend as more SPI was involved, due to a lower proportion of soluble aggregates.

Fig. $5.2 \mathrm{D}$ shows that the zeta-potential of all samples was negative at $\mathrm{pH} 7.0$, since this $\mathrm{pH}$ was above the isoelectric point of soy and whey proteins. For unheated samples, the zetapotential decreased as the proportion of SPI increased. They all decreased to a similar level (around $-28 \mathrm{mV}$ ) after heating, indicating the surface charge and electrostatic interactions of thermally-induced aggregates were similar and not affected by SPI: WPI ratios. Ryan et al. reported that heating WPI for $10 \mathrm{~min}$ at $90^{\circ} \mathrm{C}$ and pH 7.0 generated soluble WPI aggregates that had a more negative surface charge than unheated WPI [203]. Wan et al. recently found that the change in zeta-potential of SPI upon formation of aggregates was relatively small and dependent on the protein concentration during heating [23]. Fig. 5.2E shows that the surface hydrophobicity $\left(\mathbf{H}_{\mathbf{0}}\right)$ of unheated samples gradually increased with the proportion of SPI. This could be attributed to the harsh industrial processing of commercial SPI which resulted in a large extent of protein denaturation and unfolding, exposing more hydrophobic groups to the protein surface [204]. The preheating step also induced the denaturation of commercial WPI, leading to higher $\mathrm{H}_{0}$, but did not increase the $\mathrm{H}_{0}$ of samples containing a high proportion of SPI. Consistently, the intrinsic fluorescence spectra (Fig. 5.2F) of unheated samples showed a gradual red shift (increased $\lambda_{\max }$ ) as the proportion of SPI increased, indicating that Tryptophan residues were exposed to a more polar/hydrophilic environment due to unfolded protein structures [23]. After the preheating, although the $\lambda_{\max }$ of all samples further increased, the $\lambda_{\max }$ was still higher for the samples with more SPI, indicating that their thermally-induced aggregates consisted of proteins which were more unfolded. 

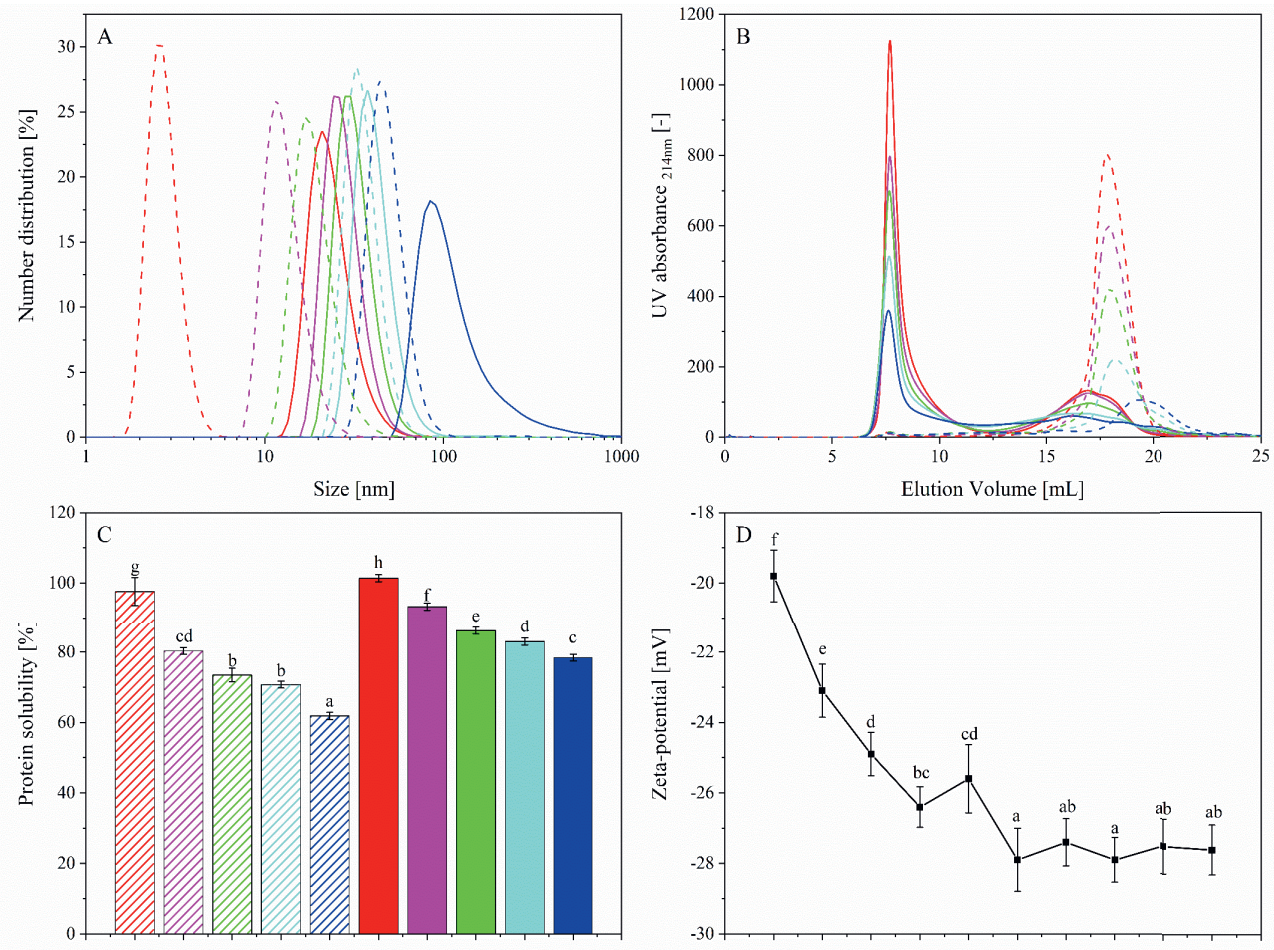

$\begin{array}{lllllllllll}0: 4 & 1: 3 & 2: 2 & 3: 1 & 4: 0 & 0: 4 & 1: 3 & 2: 2 & 3: 1 & 4: 0\end{array}$ SPI:WPI
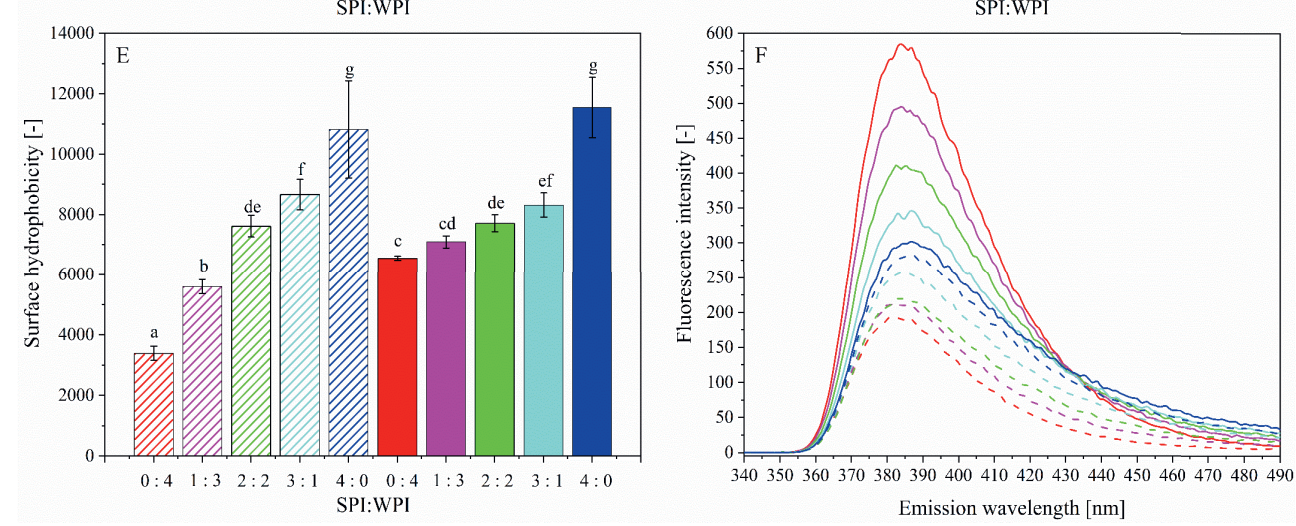

Figure 5.2 Physical and conformational properties of SPI and WPI mixtures: S:W 0:4 (red), S:W 1:3 (magenta), S:W 2:2 (green), S:W 3:1 (cyan), S:W 4:0 (dark blue). Particle size distribution (A), size exclusion chromatography of supernatant (B) and protein solubility (C), zeta potential (D), surface hydrophobicity (E), and intrinsic fluorescence spectra (F). Dashed line/bar represents non-heated mixtures (control group), while solid line/bar represents samples after preheating. Different small letters (in the same subplot) indicates that the results of different samples are significantly different $(p<0.05)$ according to ANOVA and Duncan post-Hoc test. 


\subsubsection{Acidification kinetics}

During the isothermal incubation $\left(40^{\circ} \mathrm{C}\right.$ ), the slow hydrolysis of GDL into gluconic acid releases protons, which subsequently reduce electrostatic repulsion between aggregates by protonation of charged carboxyl groups, and induce their aggregation into a threedimensional (3D) gel network [185]. It is known that the acidification kinetics of GDL depends on the protein: GDL ratio as well as the temperature. To determine how different SPI: WPI ratios would affect the acidification kinetics, we recorded the $\mathrm{pH}$ of dispersions of thermally-induced aggregates $(4 \% \mathrm{w} / \mathrm{w})$ formed at different SPI: WPI ratios at the same amount of $\mathrm{GDL}$ addition $(0.8 \% \mathrm{w} / \mathrm{w})$ and temperature $\left(40^{\circ} \mathrm{C}\right)$. As shown in Fig. $5.3_{\perp}$ all samples displayed similar acidification kinetics: the decrease in $\mathrm{pH}$ was fastest in the beginning, then slowed down and finally leveled off. Pure WPI showed a faster acidification rate than pure SPI, as it took $100 \mathrm{~min}$ for WPI to reach pH 4.5 (the pKa of glutamic acid) while $180 \mathrm{~min}$ for SPI. Similarly, for mixed aggregates a higher SPI fraction led to a slower $\mathrm{pH}$ decreasing rate. This phenomenon could be attributed to the buffering ability of soy protein [194]. The acidification rate might affect the level of structural rearrangements during gelation and thus the gel properties [205]. For example, it has been reported that bacterial-acidification-induced WPI gels showed increased hardness when the acidification rate was faster [206], which is in line with our subsequent rheology results.

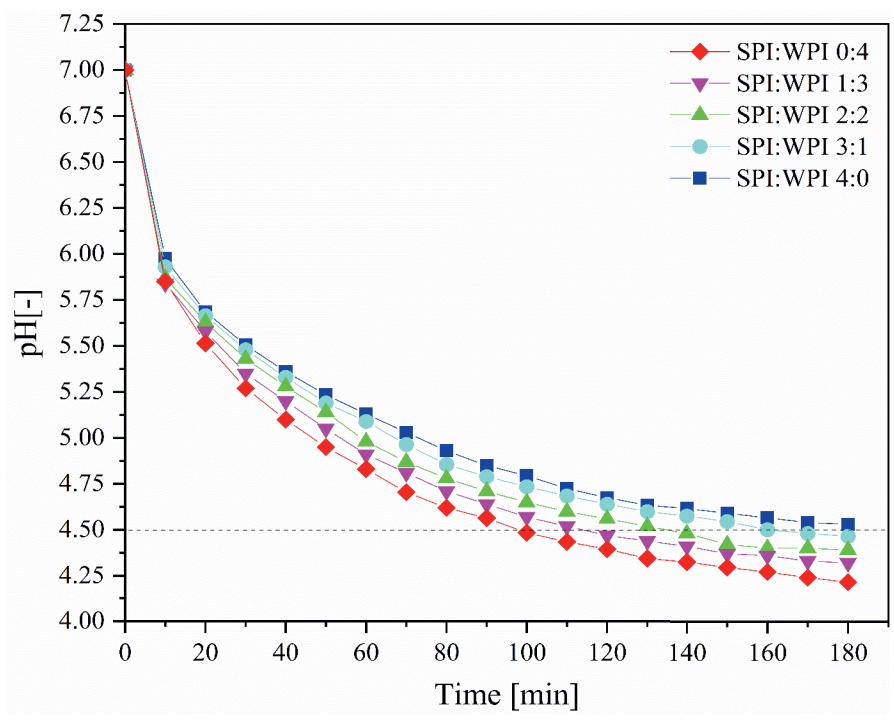

Figure 5.3 Acidification kinetics (3h) of the dispersions of thermally-induced aggregates (4\% $w / w)$. 


\subsubsection{Linear rheology}

Small amplitude oscillatory shear (SAOS) tests were used to investigate the acid-induced gelation kinetics of preheated samples and characterize the linear viscoelastic (LVE) properties of the gels. Figure 5.4 shows the results of $4 \% \mathrm{w} / \mathrm{w}$ protein samples which were used as representative samples. As shown in Fig. 5.4A, all samples displayed a similar GDLinduced gelation profile, regardless of composition and concentration (data not shown): after a short lag phase, the $\mathrm{G}^{\prime}$ and $\mathrm{G}^{\prime \prime}$ started to increase rapidly, and subsequently the gelation rate slowed down, until the moduli reached a plateau. The time when $\mathrm{G}^{\prime}$ exceeds $1 \mathrm{~Pa}$ is often taken as the gelling point of acid-induced gels [23]. It was found that all samples started to gel within $30 \mathrm{~min}$, and the gelling point was postponed when the proportion of SPI increased. We previously found that the zeta-potentials of preheated samples were similar while $\mathrm{H}_{0}$ was higher with increasing proportion of SPI. This implies that the postponed gelling point is most likely due to the slower acidification rate of SPI, resulting in a longer time for the attractive interactions such as hydrophobic interactions to overcome the electrostatic repulsive force. For gels formed at the same protein concentration, replacing WPI with SPI significantly decreased the final $\mathrm{G}^{\prime}$ at $40^{\circ} \mathrm{C}$ (Table 5.1), indicating a lower gel strength. Schmitt et al. concluded that the presence of plant proteins led to a lower gel strength compared to dairy proteins alone, because the different proteins appeared to form separate gel networks which did not interact with each other [184]. This is also consistent with our microscopy results that will be shown later.

Fig. 5.4B shows that the $\mathrm{G}^{\prime}$ and $\mathrm{G}^{\prime \prime}$ of acid-induced gels further increased when lowering the temperature from $40{ }^{\circ} \mathrm{C}$ to $4{ }^{\circ} \mathrm{C}$, which suggested reinforcement or rearrangement of the gel structure during the cooling process. According to previous studies, this increase in moduli was mainly due to the formation of hydrogen bonds $[207,208]$. Table 5.1 shows that the enhancement of $G^{\prime}$ during this cooling processing $\left(\Delta G^{\prime}=\left[G^{\prime}\left(4^{\circ} \mathrm{C}\right)-G^{\prime}\left(40{ }^{\circ} \mathrm{C}\right)\right] / G^{\prime}(40\right.$ $\left.{ }^{\circ} \mathrm{C}\right)$ ) varied with the protein composition of gels but not the protein concentration. Gels that contained more SPI showed higher $\Delta \mathrm{G}^{\prime}$, indicating this strengthening effect of cooling was more significant. This indicated that hydrogen bonds might play a more important role in maintaining the gel network of soy protein. Similarly, Ringgenberg et al. found that GDLinduced gelation of soymilk showed earlier gelation and much stiffer bonds at $7^{\circ} \mathrm{C}$ compared to $30{ }^{\circ} \mathrm{C}$, which suggested that hydrogen bonds and van der Waals interactions played a major role in strengthening the network linkages [209].

Fig. 5.4C shows that the $\mathrm{G}^{\prime}$ and $\mathrm{G}^{\prime \prime}$ of acid-induced gels at $4{ }^{\circ} \mathrm{C}$ both increased as the frequency increased from 0.1 to $10 \mathrm{~Hz}$. This indicated the formation of a continuous network and a typical physical gel structure, which normally shows a weak frequency dependence and no crossover of $\mathrm{G}^{\prime}$ and $\mathrm{G}^{\prime \prime}$ [210]. The frequency dependence of different gels is 
summarized in Table S5.1, which showed that the power law exponent of G", i.e. $n^{\prime \prime}$, was slightly higher than the exponent of $\mathrm{G}^{\prime}$, i.e. $\mathrm{n}^{\prime}$. Both $\mathrm{n}^{\prime}$ and $\mathrm{n}^{\prime \prime}$ of acid-induced gels were not significantly affected by protein concentrations, but gradually increased with the proportion of SPI. Table S5.2 also shows that the tan $\delta$ of gels at $4{ }^{\circ} \mathrm{C}$ increased with the proportion of SPI. These results indicated that the LVE properties of gels became less elastically dominated when more WPI was replaced by SPI.
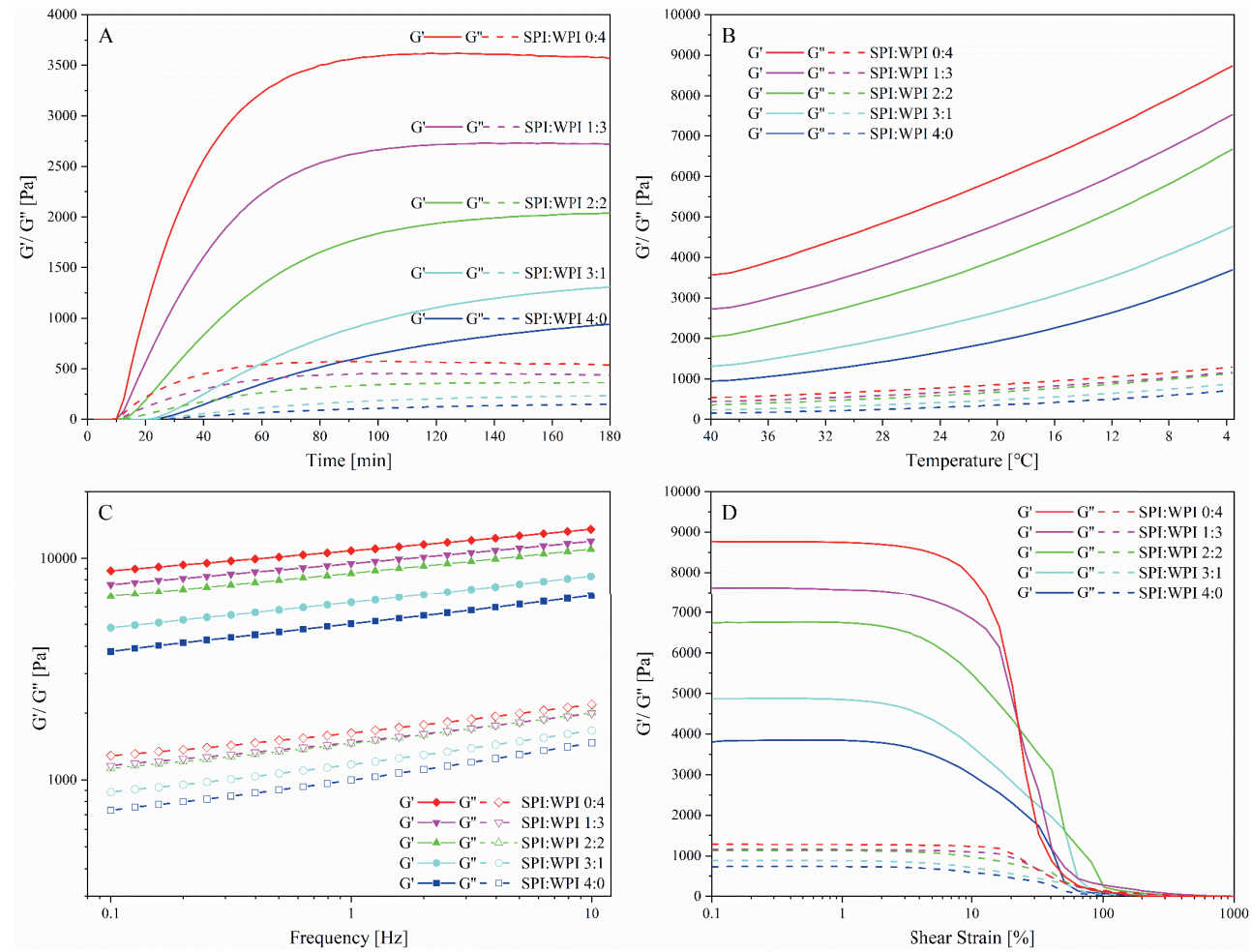

Figure 5.4 Time sweep (A) and temperature sweep (B) tests of the dispersions of thermally-induced aggregates $(4 \% \mathrm{w} / \mathrm{w})$, and frequency sweep (C) and strain sweep (D) of the resultant gels. 


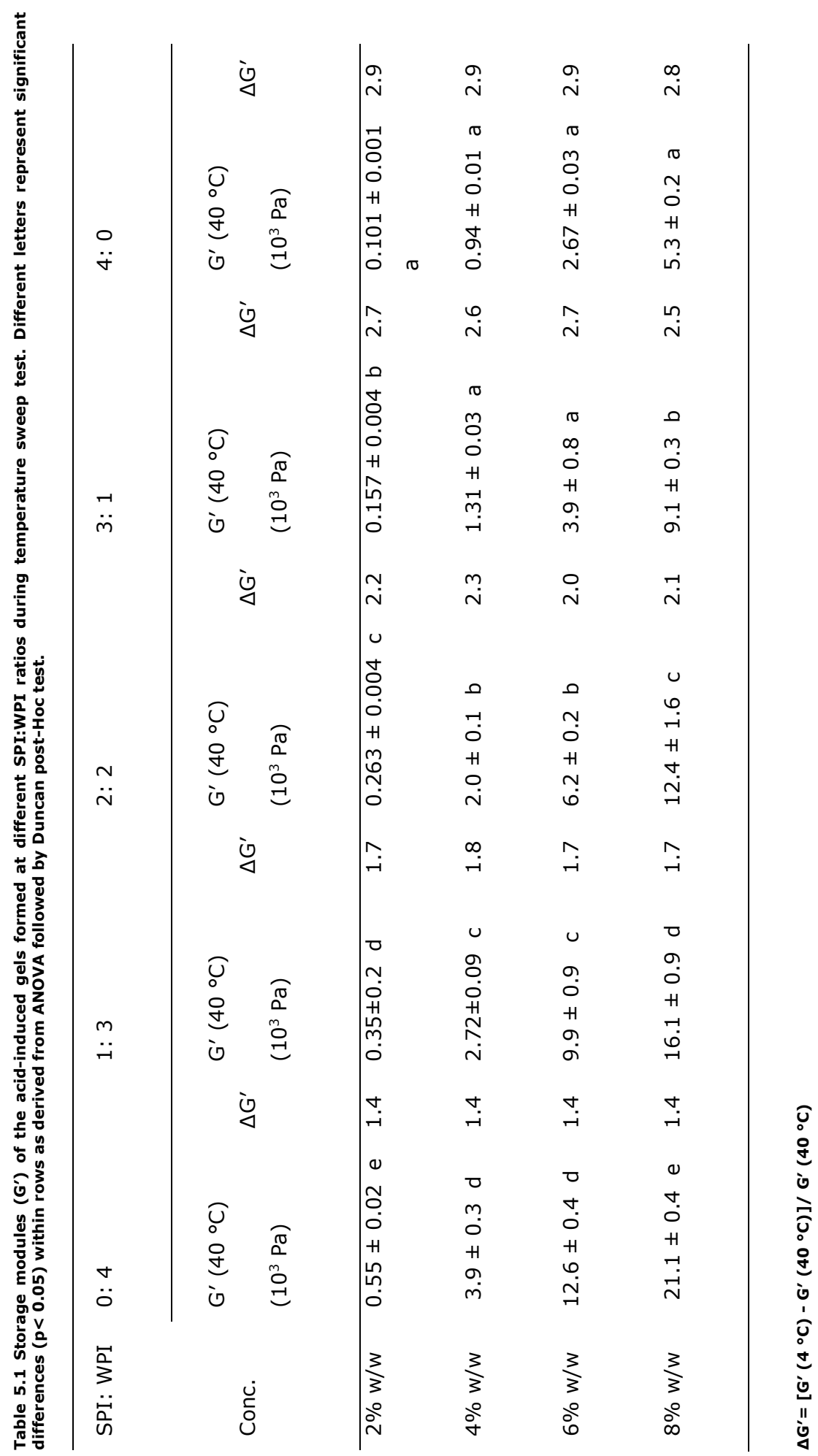




\subsubsection{Non-linear rheology}

After the frequency-sweep test, we performed a LAOS strain sweep test on these acidinduced gels to investigate their non-linear viscoelastic (NLVE) properties. Fig. 5.4D shows all gels displayed a similar strain dependence regardless of the composition and concentration (data for the latter not shown): With increasing intercycle strain amplitude ( $(\boldsymbol{\gamma}), \mathrm{G}^{\prime}$ and $\mathrm{G}^{\prime \prime}$ first displayed a clear plateau, representing the linear viscoelastic (LVE) regime. When yo exceed a certain value, both $\mathrm{G}^{\prime}$ and $\mathrm{G}^{\prime \prime}$ decreased gradually, which is known as shear strain softening (Type I) behavior. This non-linear rheological behavior has been explained by a decreased creation rate and increased loss rate of gel network-bonds when $Y_{0}$ is increased [24]. Similar behavior was also observed for the acid-induced gels formed by pure soy protein [144] and peanut protein [211].

The gels displayed similar strain sweep profiles, but the range of their LVE regime, i.e. the critical linear strain $(\boldsymbol{\gamma} \boldsymbol{c})$, significantly changed with protein concentrations and compositions, as shown in Table 5.2. When the gels were formed at the same protein concentration, $y_{c}$ generally decreased as the proportion of SPI increased, indicating that replacing WPI with SPI made the acid-induced gels less ductile. When the gels were formed at the same SPI: WPI ratio, the $\gamma_{c}$ dependence on protein concentration was different for the various ratios, which will be further discussed in the next section.

Table 5.2 Critical linear strain $(\gamma c)$ of the acid-induced gels formed at different SPI:WPI ratios.

\begin{tabular}{|c|c|c|c|c|c|}
\hline SPI: WPI & $0: 4$ & $1: 3$ & $2: 2$ & $3: 1$ & $4: 0$ \\
\hline Conc. & $Y_{c}[\%]$ & $Y_{c}[\%]$ & $\gamma_{c}[\%]$ & $Y_{c}[\%]$ & $Y_{c}[\%]$ \\
\hline $2 \% \mathrm{w} / \mathrm{w}$ & $42.2 \pm 2.2 \mathrm{c}$ & $11.3 \pm 2.8 \mathrm{~b}$ & $6.0 \pm 0.5 \mathrm{a}$ & $5.4 \pm 0.4 a$ & $5.6 \pm 0.0 a$ \\
\hline $4 \% \mathrm{w} / \mathrm{w}$ & $9.2 \pm 0.7 \mathrm{~d}$ & $8.0 \pm 0.2 \mathrm{c}$ & $5.5 \pm 0.6 b$ & $2.2 \pm 0.1 \mathrm{a}$ & $2.4 \pm 0.1$ \\
\hline $6 \% \mathrm{w} / \mathrm{w}$ & $7.9 \pm 0.0 \mathrm{~d}$ & $6.8 \pm 0.0 \mathrm{c}$ & $5.9 \pm 0.3 b$ & $3.2 \pm 1.9 \mathrm{a}$ & $2.2 \pm 0.2$ \\
\hline $8 \% \mathrm{w} / \mathrm{w}$ & $6.8 \pm 0.5 \mathrm{c}$ & $6.4 \pm 0.4 \mathrm{c}$ & $5.9 \pm 0.1 b c$ & $4.8 \pm 1.3 b$ & $1.7 \pm 0.7$ \\
\hline
\end{tabular}




\section{Fractal scaling model}

The concentration dependence of $\mathrm{G}^{\prime}$ and $\gamma_{c}$ of protein gels are often given by a power-law relationship, i.e. $G^{\prime} \sim C^{A}, Y_{c} \sim C^{B}$, as shown in Fig. S5.3. Although the $G^{\prime}\left(4^{\circ} \mathrm{C}\right)$ of all acidinduced gels increased with protein concentration, the exponent $A$ varied with different SPI: WPI ratios, as shown in Table 5.3. Pure WPI gels displayed an exponent $A$ of 2.65 which is similar to the results $(2.75 \pm 0.20)$ reported by Alting et al. [212]. As the proportion of SPI increased, this exponent $A$ gradually increased to 2.96 (for pure SPI gels). The increased exponent $A$ indicated that proteins were used less efficiently in the gelation and distributed more heterogeneously in the structure, which is consistent with the gel morphology observed later on (Fig. 5.7). For pure WPI and SPI gels, their $\gamma_{c}$ decreased with protein concentration, following a power-law model with an exponent $B$ equal to -1.34 and -1.05 , respectively. When the gels were formed by WPI and SPI mixtures, the concentration dependence of $\gamma_{c}$ became rather different. For the gels formed at SPI: WPI ratio of $1: 3$, the $\gamma_{c}$ decreased with protein concentration, still following a power-law model but with a less negative exponent $B(-0.42)$. For the gels formed at SPI: WPI ratio of $2: 2$, the $\gamma_{c}$ appeared to be independent of the protein concentration. While for the gels formed at SPI: WPI ratio of $3: 1$, the $y_{c}$ first decreased and then increased with protein concentrations.

Shih et al. has developed a scaling theory for the rheological properties of colloidal gels by considering the gel network as a collection of fractal protein flocs which are closely packed throughout the sample [26]. In their theory, gel structures are classified into a strong-link regime and a weak-link regime. In the strong-link regime, the inter-floc links are stronger than the intra-floc links, and $\gamma_{c}$ decreases with increasing protein concentration, while in the weak-link regime, the situation is opposite. Our results indicated that although acid-induced gels formed by pure WPI and SPI could both belong to the strong-link regime, the hybrid gels formed by their mixtures could not be clearly classified as either strong- or weak-link. In these hybrid gels, the interactions and the fractal nature of the flocs seemed to depend on the SPI: WPI ratio. Wu \& Morbidelli further develop Shih's fractal model by introducing a new parameter $a \in[0,1]$ that indicates the relative importance of inter- and intra-floc links in gels. $a$ value of 0 indicates ideal strong-link regime and $a$ value of 1 indicates ideal weaklink regime [27]. With this model an estimate of the fractal dimension $\left(\boldsymbol{D}_{\boldsymbol{f}}\right)$ of the flocs can be obtained based on the concentration dependence of $\mathrm{G}^{\prime}$ and $\gamma_{c}$ : in this extended model $A$ $=[(d-2)+(2+x)(1-a)] /\left(d-D_{f}\right)$, and $B=[1-(2+x)(1-a)] /\left(d-D_{f}\right)$. Here $x$ represents the fractal dimension of the backbone of the flocs (ranging from 1.0 to 1.3 for colloidal gels and assumed to be 1.1 in this study). Table 5.3 shows that the calculated $D_{f}$ and $a$ gradually increased for the gels in the order of pure WPI, pure SPI, S:W 1:3 and S:W 2:2. The higher $D_{f}$ indicated that the constituent flocs had more compact structures. Meanwhile, only for 
pure WPI gels the $a$ value was close to zero (0.02), indicating only pure WPI gels were of the strong-link type, while other gels that contained SPI were in the intermediate regime.

Table 5.3 The protein concentration $(C)$ dependence of storage modules $\left(G^{\prime}\right)$ and critical linear strain $\left(Y_{c}\right)$ of the acid-induced gels formed at different SPI:WPI ratios, and the fractal dimension $\left(D_{f}\right)$ calculated based on scaling theory of Wu et al. (1) The Euclidean dimension $d$ of the gel system is 3 and the backbone fractal dimension $x$ of the flocs is assumed to be 1.1 .

\begin{tabular}{lllll}
\hline SPI: WPI & $\mathrm{G}^{\prime} \propto \mathrm{C}^{\mathrm{A}}$ & $\mathrm{Yc} \propto \mathrm{C}^{\mathrm{B}}$ & $\mathrm{A}=\frac{(d-2)+(2+\alpha)(1-\alpha)}{d-D_{f}}{ }^{1}$ \\
& & & $\mathrm{~B}=\frac{1-(2+x)(1-\alpha)}{d-D_{f}}$ & \\
\hline $4^{\circ} \mathrm{C}$ & $\mathrm{A}$ & $\mathrm{B}$ & $D_{f}$ & $a$ \\
$0: 4$ & 2.65 & -1.34 & 1.47 & 0.02 \\
$1: 3$ & 2.78 & -0.42 & 2.15 & 0.56 \\
$2: 2$ & 2.84 & 0.01 & & 0.68 \\
$3: 1$ & 2.93 & -- & 2.30 & -- \\
$4: 0$ & 2.96 & -1.05 & -- & 0.32 \\
\hline
\end{tabular}

\section{Lissajous plots}

Since $\mathrm{G}^{\prime}$ and $\mathrm{G}^{\prime \prime}$ are based only on the first harmonic contribution to the stress response and may have ambiguous physical meaning in the NLVE regime [139], the intracycle oscillatory response at various intercycle strains $(\boldsymbol{\gamma} \boldsymbol{o})$ or strain rates $\left(\dot{\gamma}_{0}\right)$ were analyzed using Lissajous plots. The elastic Lissajous plots (Fig. 5.5A) and viscous Lissajous plots (Fig. 5.5B) of $4 \% \mathrm{w} / \mathrm{w}$ gels are constructed by plotting stress $(\boldsymbol{\sigma})$ vs. intracycle strain $(\boldsymbol{\gamma})$ and stress $(\sigma)$ vs. intracycle strain rate $(\dot{\gamma})$, respectively. To better illustrate the elastic and viscous contribution to the total stress $(\sigma)$, the decomposed elastic $\left(\boldsymbol{\sigma}_{\mathbf{e}}\right)$ and viscous stress $\left(\boldsymbol{\sigma}_{\mathbf{v}}\right)$ were also plotted within the loops of the elastic and viscous Lissajous plots, respectively.

As shown in Fig. 5.5A, at a yo equal to $0.1 \%$, all gels $(4 \% \mathrm{w} / \mathrm{w})$ displayed a narrow and elliptical elastic Lissajous plot, and the $\sigma_{\mathrm{e}}$ displayed a linear dependence on $\mathrm{y}$ (see straight dashed line). This demonstrated that the deformation amplitude in our SAOS test, i.e. $0.1 \%$, was sufficiently small to ensure all the gels were within the LVE regime. As the yo increased to $10.2 \%$, the shape of the elastic Lissajous plots started to deviate from an elliptic shape 
due to the impact of higher harmonics presented in the NLVE regime. This distortion was more obvious when the proportion of SPI was higher, which was consistent with our previous results that increasing the SPI: WPI ratio shortened the range of the LVE regime (Table 5.2). When $y_{0}$ increased to $40.8 \%$, the shapes of the elastic Lissajous plots differed significantly. For the pure WPI gel, the plots were significantly wider than for the other samples and already started to change into a more rhomboidal shape. The area enclosed by the loop and the difference between $\sigma$ and $\sigma_{e}$ both increased significantly, indicating considerable structure breakdown [150]. For hybrid gels formed by WPI and SPI, the loops became progressively narrower with increasing SPI: WPI ratio. The Lissajous plots of these gels displayed a narrow and inverted sigmoidal shape with a steep upswing near maximum $Y$. The smaller enclosed area of elastic Lissajous plots indicated less dissipated energy per cycle, and the narrower gap between $\sigma$ and $\sigma_{e}$ indicated that the stress response is relatively more elastic dominated. These results suggested that although WPI gels displayed higher stiffness $\left(G^{\prime}\right)$ than hybrid gels and SPI gels in the LVE regime, they are more brittle, as the transition to a fully plastic behavior occurred at much lower strains. Compared to the relatively straight $\sigma_{e}$ curves in the LVE regime, the lower slope of $\sigma_{e}$ curves at small intracycle strain $[-0.5<y<0.5]$ indicates gels have less ability to support elastic stress due to the decreasing number of network bonds, which can be associated with the overall intercycle strain softening observed in Fig. 5.4D. The steep slope at higher intracycle strain $[\gamma<-0.5$ or $y>0.5$ ] could have resulted from the stretching of dissociated flocs, which manifested in an apparent intracycle stiffening [166].

When the $y_{0}$ increased to $128 \%$, the Lissajous plots of pure WPI gels had already changed into a nearly rectangular shape and did not change much further up to the maximum amplitude, i.e. $1000 \%$. This near plastic behavior $[150,167]$ is evident from an intracycle sequence of (starting from the lower-left corner $(y=-1)$ of this curve, in a clockwise direction) elastic straining (the vertical part), yielding, flow (the horizontal part), and recovery. In contrast, this transition to plastic behavior proceeded in a much more gradual way for gels that contained SPI. For pure SPI gels, the Lissajous plots changed into a near rhomboidal shape at $y_{0}=128 \%$, and for the hybrid gel formed at SPI: WPI ratio of $1: 3$, their Lissajous plots had an inverted sigmoidal shape even up to a deformation amplitude of $510 \%$. In addition, while the $\sigma_{e}$ of pure WPI gels was near zero for almost the entire cycle when $y_{0}$ reached $204 \%$, one could still observe nonzero values for $\sigma_{e}$ when the other gels were subjected to the maximum $y_{0}(1020 \%)$, a sign there was still residual elasticity in the material. That the gels containing SPI showed a slower transition towards plastic properties and still retained an elastic contribution to the response at higher strain, could be another indication of their coarser network structures, since coarser structures are less prone to a complete transition towards plastic behavior due to increased structural flexibility [149]. The 
higher flexibility could lead to a more gradual decrease in bond numbers, thus some elastic responses can still be detected at higher strain [149].

As a complement to the elastic Lissajous plots, viscous Lissajous plots were shown in Fig. 5.5B. In contrast to the former, the enclosed area of viscous Lissajous plots represents the stored energy (proportional to $\mathrm{G}^{\prime}$ ) per cycle. When $y_{0}$ is $0.1 \%$, all gels displayed circular viscous Lissajous plots, showing that all gels displayed predominantly elastic behavior in the LVE regime. As $y_{0}$ increased, the shape of viscous Lissajous gradually changed into a sigmoidal shape with a decreased enclosed area, which indicated the decay of elasticity. Obviously, this decay started at lower strain rates and proceeded more abruptly in pure WPI gels compared to the other gels. For the gels that contained SPI, a distinct decrease in the stress can be observed near the maximum normalized intracycle strain rate ( $\dot{\gamma}=-1$ or 1 ), which indicated strong intracycle shear thinning behavior. At high deformation amplitude ( $y_{0}$ $\geq 204 \%$ ), secondary loops were clearly observed in the viscous Lissajous plots of the pure WPI gel, which could be explained by a coupling of the elasticity of sample with instrument inertia $[147,176]$.

Overall, we found soy protein plays a dominant role in the non-linear rheological behavior of WPI and SPI hybrid gels, as the evolution of both elastic and viscous Lissajous plots of hybrid gels are more similar to that of pure SPI gels, regardless of the ratios. Even when only $25 \%$ WPI was replaced with SPI, the NLVE properties of acid-induced gels changed significantly. This phenomenon can be also found in the gels formed at other concentrations (Fig. S5.4). Although gels with similar strength $\left(\mathrm{G}^{\prime}\right)$ can be obtained by two proteins with different concentrations (e.g. $4 \% \mathrm{w} / \mathrm{w}$ WPI and $6 \% \mathrm{w} / \mathrm{w}$ SPI) (Fig. S5.3), their NLVE properties still differ a lot (Fig. S5.5), which demonstrates that the rheological behavior beyond the LVE regime might be a better reflection of the microstructural differences between samples. 
Figure 5.5 Elastic Lissajous plots (A) and viscous Lissajous plots (B) of normalized stress (s/Smax) versus normalized strain $(\mathrm{g} / \mathrm{go})$ or normalized strain rate $\left(\dot{\gamma} / \dot{\gamma}_{0}\right)$, for acid-induced gels $(4 \% \mathrm{w} / \mathrm{w})$ formed at different SPI:WPI ratios; S:W 0:4 (red), S:W 1:3 (magenta), S:W 2:2 (green), S:W 3:1 (cyan), S:W 4:0 (dark blue). Solid lines indicate the total stress $(\sigma)$ and dashed lines within the loop indicates the decomposed elastic stress $\left(\sigma_{e}\right)$ and viscous stress $\left(\sigma_{v}\right)$.

(A)
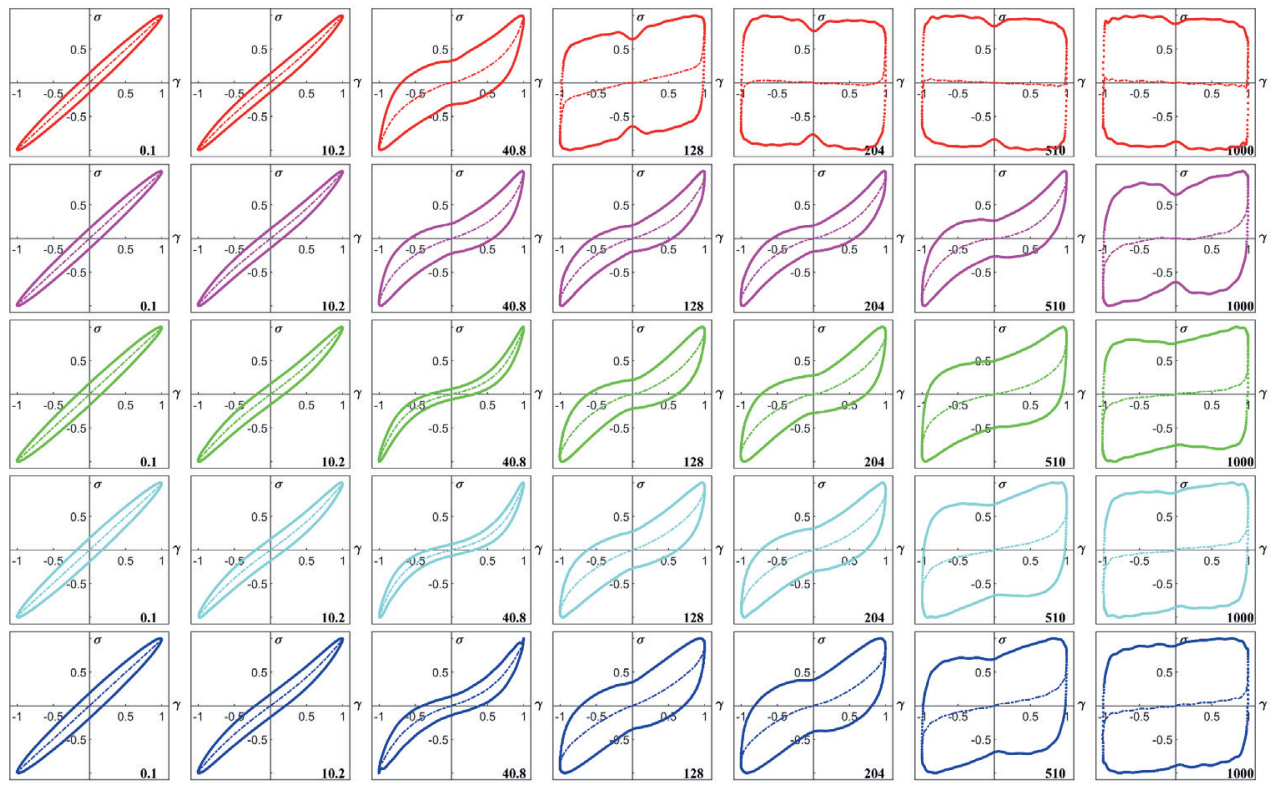

(B)
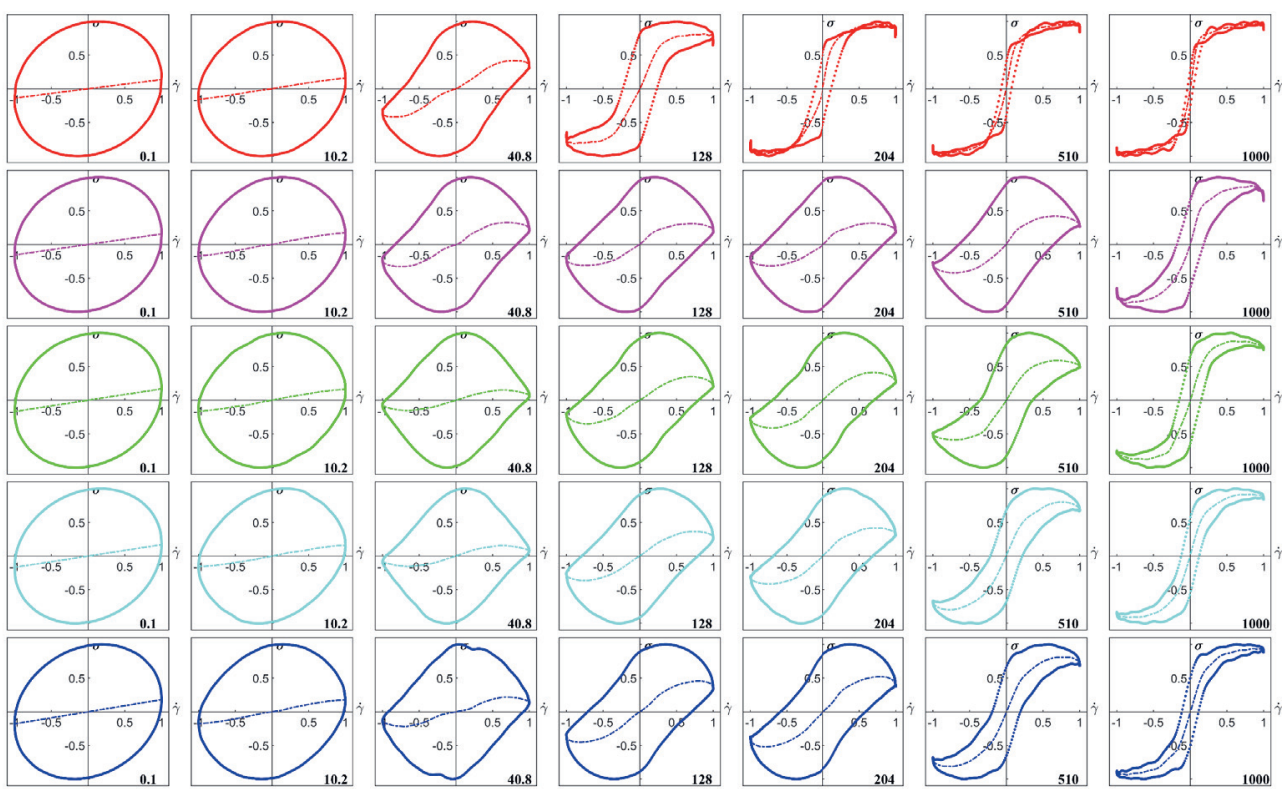


\subsubsection{Water holding capacity and gel solubility}

Fig. 5.6A shows the effects of SPI: WPI ratio on the water holding capacity (WHC) of acidinduced gel $(4 \% \mathrm{~W} / \mathrm{w})$. The WHC of pure WPI gels was about $88.7 \%$, which was almost three times the WHC of pure SPI gels (33.8\%). Increasing the proportion of SPI significantly decreased the WHC of hybrid gels. The WHC of gels has been found closely related to the rheological properties as well as the microstructures of gels. Stiffer gels (with higher $\mathrm{G}^{\prime}$ ) generally had larger WHC than less stiff gels, since under the same centrifugal force the stiff gels would experience a lower extent of compression compared to the weaker ones, and hence less water was expelled from the former. This is consistent with our SAOS results. Urbonaite et al. reported that the homogeneity of a gel microstructure significantly contributes to WHC [213], which is also consistent with the microscopy images of the gel microstructure as shown later (Fig. 5.7).

Figure 5.6 Water holding capacity and gel solubility of acid-induced gels (4\% w/w) formed at different SPI:WPI ratios. Different letters (in the same subplot) indicate the results for different samples have significant differences $(p<0.05)$ according to ANOVA and Duncan post-Hoc test.

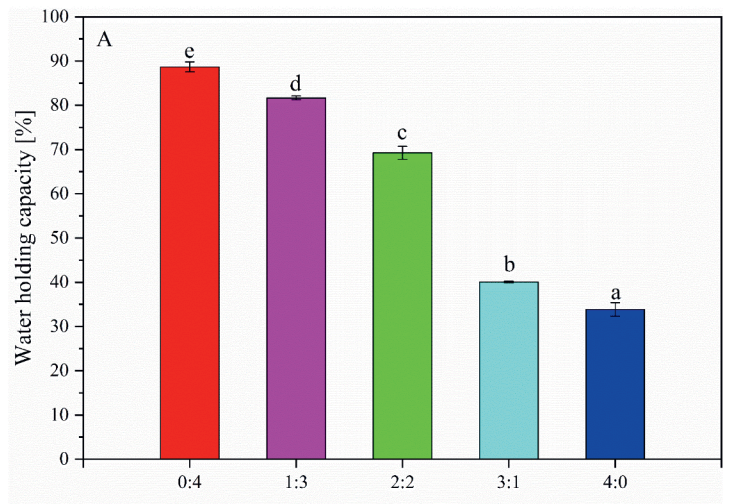

SPI:WPI

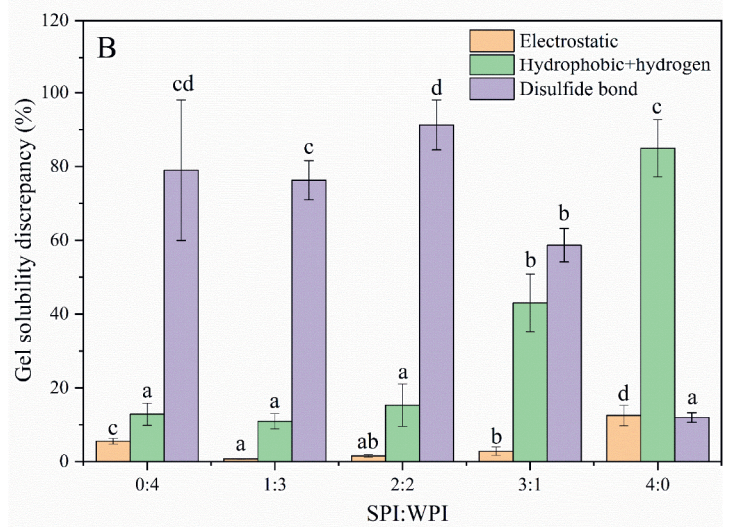


Evaluating the gel solubility in the solvents $S_{A}, S_{B}, S_{C}$, and $S_{D}$ (see Section 5.2.7) reveals the types of protein interactions and their relative importance in different gels. As shown in Fig. 5.6B, both covalent and non-covalent interactions are all involved in the acid-induced gels formed by SPI and WPI, which is consistent with previous studies $[214,215]$. For pure WPI gels, the contribution of disulfide bonds was significantly more important than hydrophobic interactions and hydrogen bonds, and the contribution of electrostatic interactions was the lowest. This is consistent with the study of Alting et al., who added thiol blocking agents to whey protein aggregate dispersions before acid-induced gelation, and observed a dramatic decrease (5 to 10 -fold) in the gel hardness [178]. Partially replacing WPI with SPI (S: W of $1: 3$ or 2: 2) did not change the relative importance of these interactions in the gels, except for the electrostatic interactions which slightly decreased. As SPI became abundant in the gels ( $\mathrm{S}: \mathrm{W}>2: 2$ ), the contribution of non-covalent interactions (hydrogen bonds, hydrophobic and electrostatic interactions) significantly increased, while the importance of disulfide bonds significantly decreased, which could be a sign of transition of the dominant gel network as shown later. The increased extent of hydrophobic interactions could be related to the higher surface hydrophobicity of SPI aggregates (Fig. $5.2 \mathrm{E})$. An increased extent of hydrogen bonding would explain the stronger increase of $\mathrm{G}^{\prime}$ (higher $\Delta G^{\prime}$ ) when these gels were subjected to a cooling step, since hydrogen bonds are known to be enhanced at a lower temperature. Although the increased noncovalent interactions are beneficial to the formation of disulfide bonds during acidic gelation [178], the much lower composition of sulfur-containing amino acids (Met and Cys) of soy protein compared to whey protein, could substantially limit the formation of disulfide bonds in these SPI-abundant gels [216].

\subsubsection{Gel morphology}

Understanding the relationship between the rheological properties of materials and their microstructures is essential for designing new food products but also a long-term challenge to rheologists. Here, we applied different microscopy techniques to observe the gel microstructure. The first row of Fig. 5.7 shows 3D images of gel microstructures made using multiphoton laser scanning microscopy (MLSM) with a magnification of 40 . Here the SPI and WPI were stained by different dyes and thus are shown in different colors: SPI in green while WPI in red. It can be seen that pure WPI gels showed a denser and more homogenous structure than pure SPI gels. In WPI gels, the constituent flocs appear to be smaller and more evenly distributed in 3D space, while in SPI gels, the flocs were larger and more irregularly shaped, and much larger pores and gaps were observed. Our previous results showed that preheated SPI had a lower amount of soluble aggregates and a postponed onset of gelation, which may not be sufficient for a denser and well-arranged network to form. This coarser and looser structure explained why SPI gels showed lower G', shorter LVE 
range, and worse WHC than WPI gels, since their less strongly connected and more porous network could be less resilient to deformation, and the water that was originally present in these large gaps and pores could more readily flow out. Replacing WPI with SPI significantly coarsened the gel microstructure, and the gaps and pores increased as the SPI: WPI ratio increased. Similarly, Roesch et al. also found that the network structures of soy/skim milk mixed GDL-induced gels showed larger pore sizes because of the presence of soy proteins [194]. As mentioned, these coarse networks could explain why gels that contained SPI were less prone to a complete transition towards plastic properties. MLSM also revealed that SPI and WPI appeared to form independent gel networks (Fig. S5.6). When the SPI: WPI ratio was 1: 3, the network of the hybrid gels was mainly formed by WPI, with SPI embedded as particulate fillers. When the SPI: WPI ratio was $3: 1$, the situation appeared to be the opposite. When the SPI: WPI ratio was 2: 2 , the main network was hard to identify, as the occupancy of each network in 3D space seems to be roughly equal.

The second row of Fig. 5.7 shows the images obtained by scanning electron microscopy (SEM) with a much higher magnification of 50000, which provided more detailed information of a cross-section through the gel. SEM shows that all the flocs in the gels consisted of roughly spherical protein particulates. Consistent with the MLSM images, the flocs in pure WPI gel were relatively small and more homogenously distributed. For the gels that contained SPI, the flocs have rougher, more elongated and irregularly branched structures, which appear to be linked to each other by only a few strands, especially obvious when the SPI: WPI ratio was 1: 3 and 2:2. These results agreed with their higher $D_{f}$ and $a$ as analyzed by fractal scaling theory. Under deformation, these thin strands could be easily broken, which led to a shorter LVE range. The dissociated flocs would move in the flow field and subsequently be stretched at higher strain, which would explain the apparent intracycle hardening we observed. In addition, since noncovalent interactions played a more important role in samples containing SPI, bonds between flocs could also be more easily reformed. All these features could allow these gels to retain more elasticity even at very high strain, as shown in their Lissajous plots (Fig. 5.5A). Although the dense, homogeneous, and stronglinked structure allowed WPI gels to resist higher intercycle strain, once the strain exceeds the critical strain, cracks in the microstructure will form more quickly and evenly, leading to a more complete structural breakdown and plastic behavior. These results supported our findings that hybrid gels displayed more similar NLVE properties with pure SPI gels. 


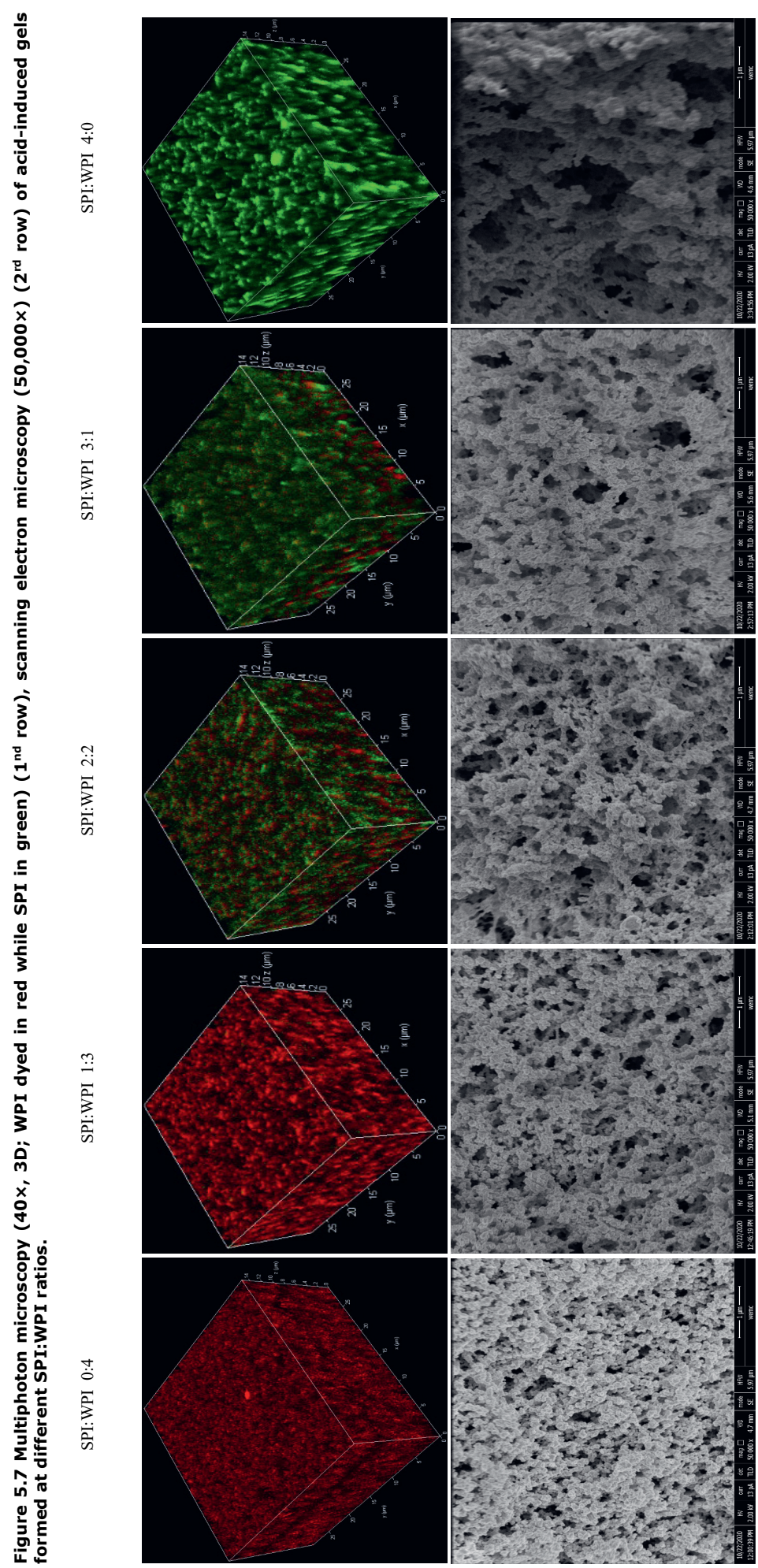




\subsection{Conclusion}

Acid (GDL)-induced gels with different rheological and microstructural properties can be obtained by mixing commercial SPI and WPI at different ratios and concentrations. Replacing WPI with SPI decreased the stiffness (lower $\mathrm{G}^{\prime}$ ) and stretchability (lower $\gamma_{c}$ ) of acid-induced gels in the linear viscoelastic (LVE) regime, but the gels had a relatively more elastic response in the non-linear viscoelastic regime (NLVE) and a more gradual transition to plastic behavior. The changes in the rheological properties can be correlated to differences in the gel microstructure. Replacing WPI with SPI decreased the proportion of soluble aggregates formed in the preheating step, slowed down the acidification rate of GDL, and postponed the gelling points. As a result, hybrid gels and pure SPI gels displayed coarser and more porous microstructures with lower water holding capacity, compared to pure WPI gels. Replacing WPI with SPI also changed the state of constituent flocs. The flocs became larger, with rougher, more elongated, and branched structures. The inter-floc links became less strong and the interactions responsible for gel formation shifted from mainly disulfide bonds to mainly noncovalent interactions. Overall, these results provide useful information for replacing whey proteins with soy protein in traditional acid-induced gel foods since their sensory attributes, especially those related to chewing down and residual sensations, are more closely correlated with behavior in the NLVE regime. Further strides could be made to link the current results, especially the LAOS data, with the sensory properties of these gels. 


\subsection{Supplementary materials}

Figure S5.1. Size exclusion chromatography (SEC) of standard proteins (A) and the calibration curve (B, C). Seven standard samples were used: Blue dextran (MW $2000 \mathrm{kDa}$ ), Thyroglobulin (MW $669 \mathrm{kDa}$ ), Apoferritin (MW 443 kDa), B-Amylase (MW 200 kDa), Alcohol dehydrogenase (MW 150 kDa), BSA (66 kDa), Carbonic anhydrase (MW 29 kDa).
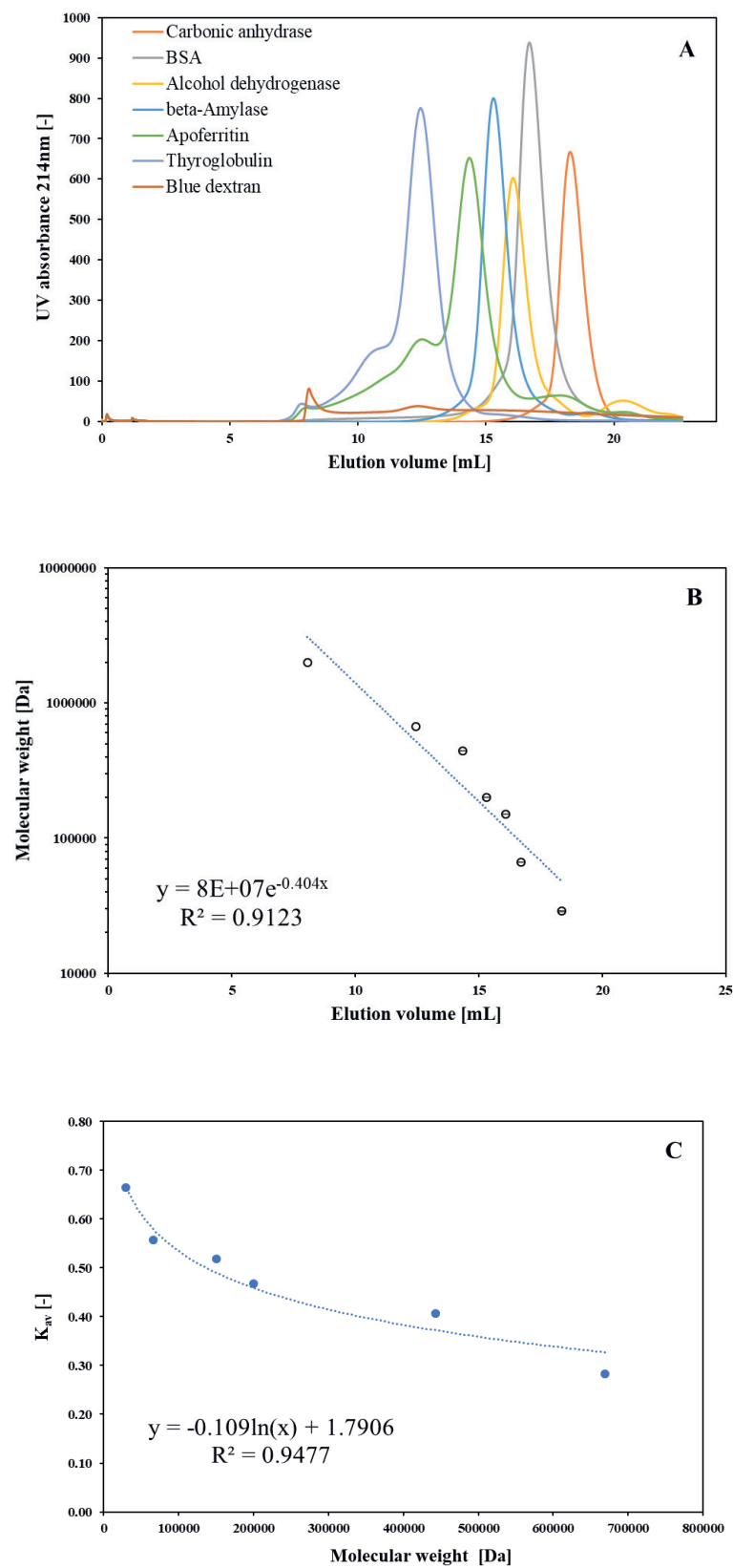
Figure S5.2. Linear relationship between protein solubility and enclosed area of SEC peaks (Gray symbols represent unheated samples, while orange symbols represent preheated samples).

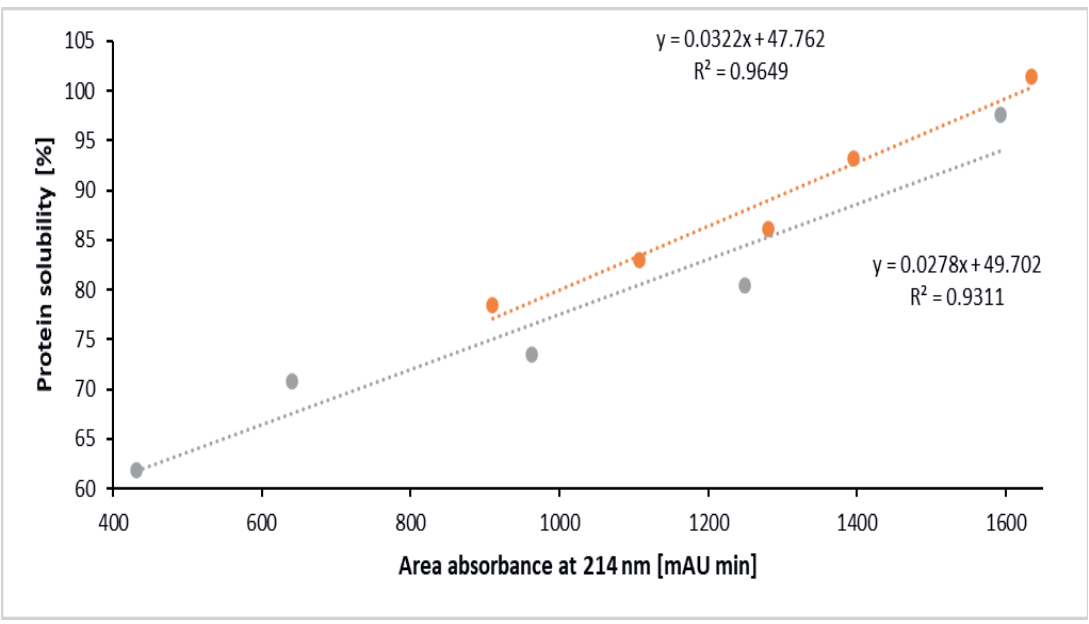

Figure S5.3. Concentration dependency of storage module $\left(G^{\prime}\right)(A)$ and linear critical strain $(Y c)(B)$ of acidinduced gels formed at different SPI:WPI ratios.
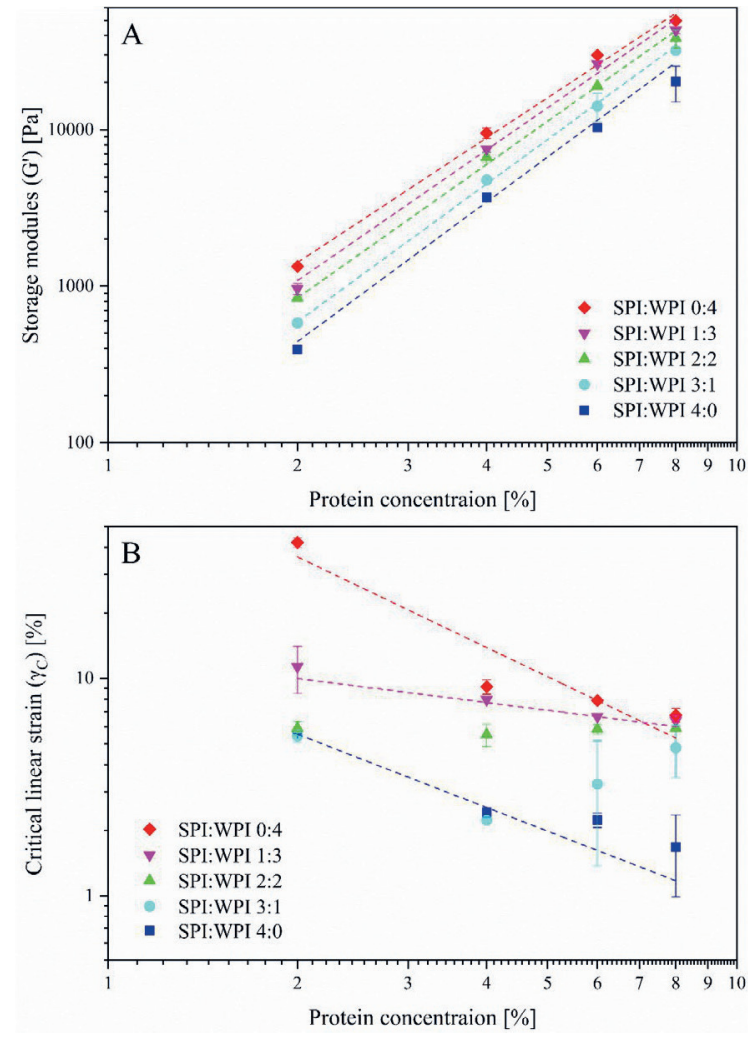
Figure S5.4 Elastic Lissajous plots of normalized stress (s/smax) versus normalized strain (g/g0) for acidinduced gels formed at $2 \% \mathrm{w} / \mathrm{w}(\mathrm{A}), 6 \% \mathrm{w} / \mathrm{w}(B)$, and $8 \% \mathrm{w} / \mathrm{w}$ (C) with different SPI:WPI ratios; S:W 0:4 (red), S:W 1:3 (magenta), S:W 2:2 (green), S:W 3:1 (cyan), S:W 4:0 (dark blue). Solid lines indicates the total stress $(\sigma)$ and dashed lines within the loop indicates the decomposed elastic stress $(\sigma e)$ and viscous stress ( $\sigma v)$.

(A)
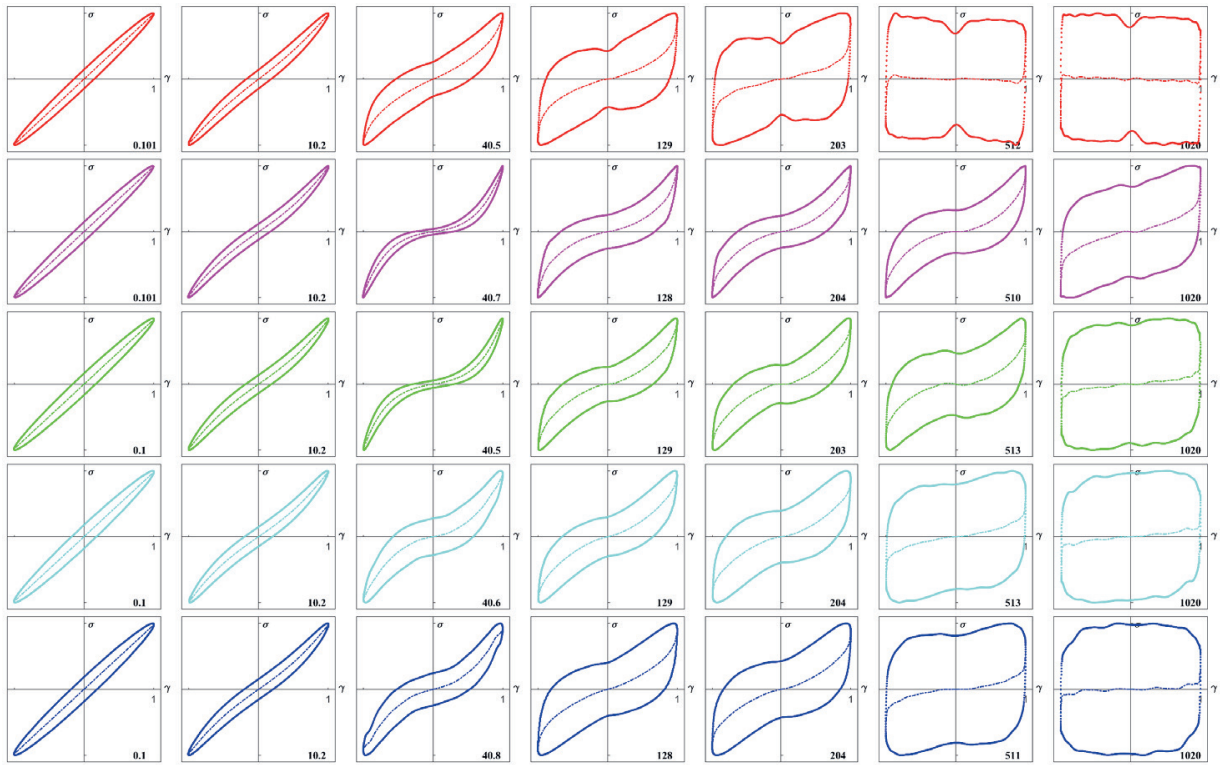

(B)
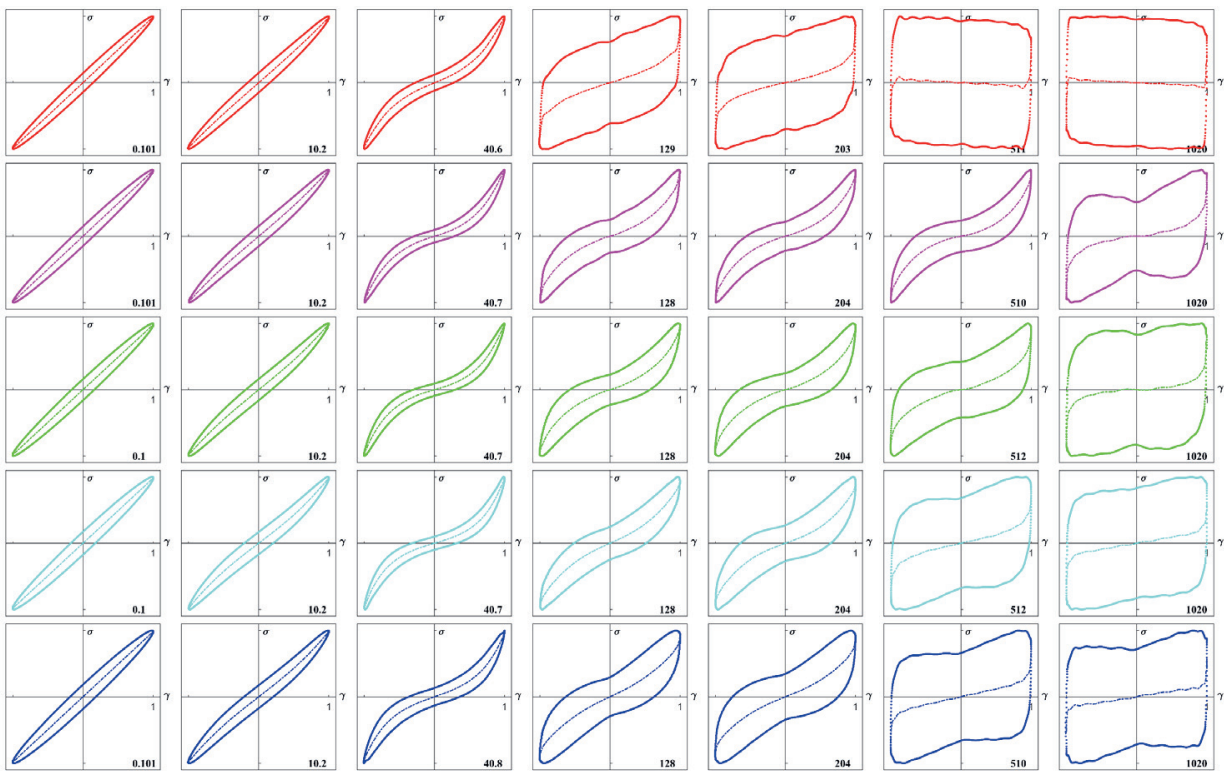

(C) 

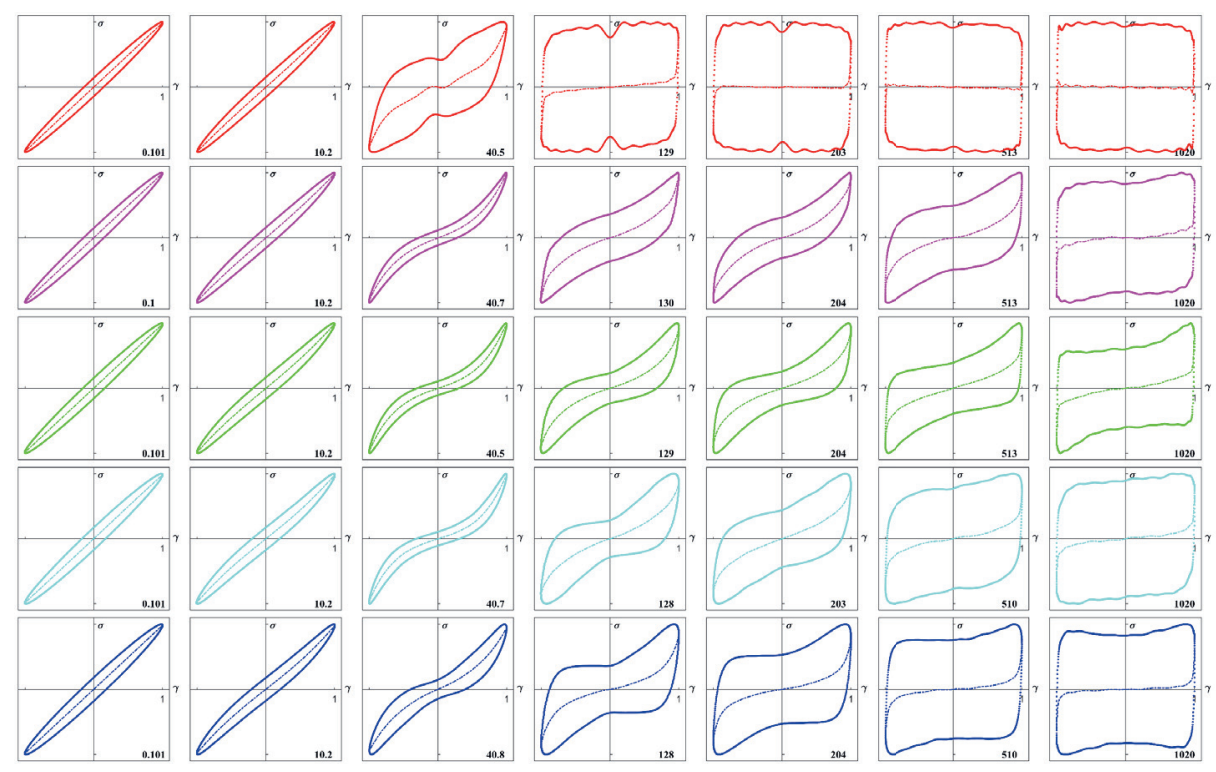

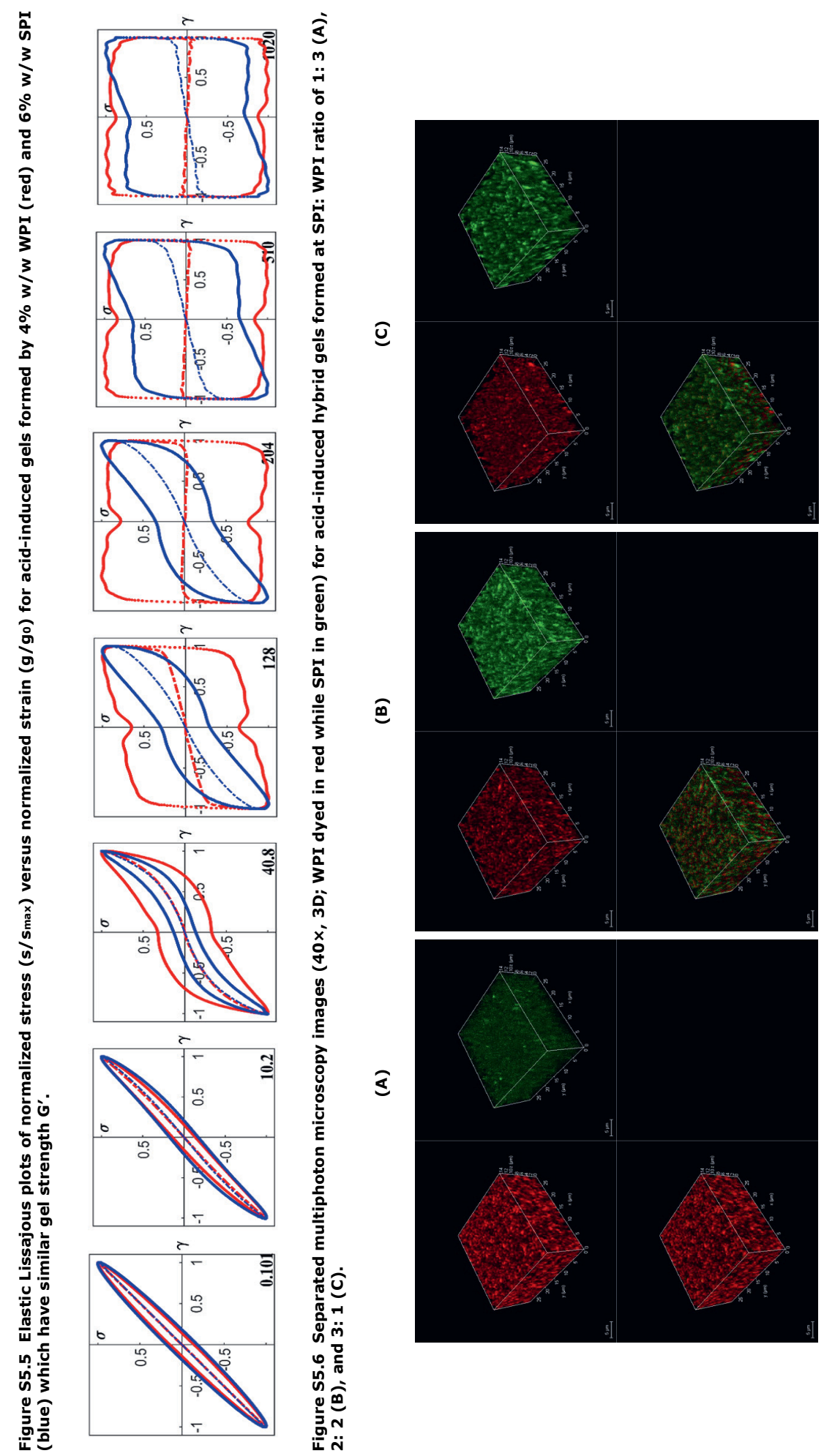


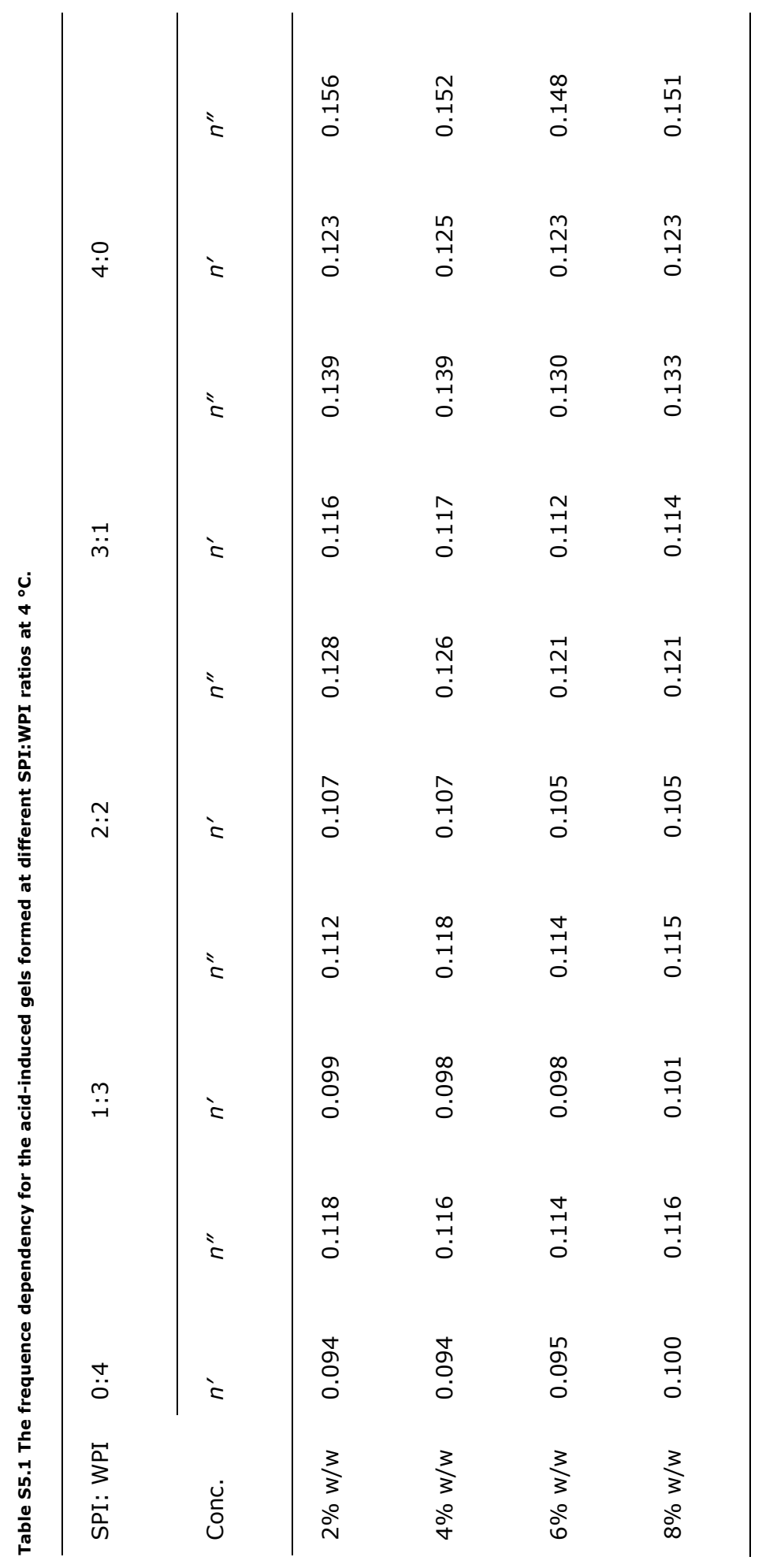




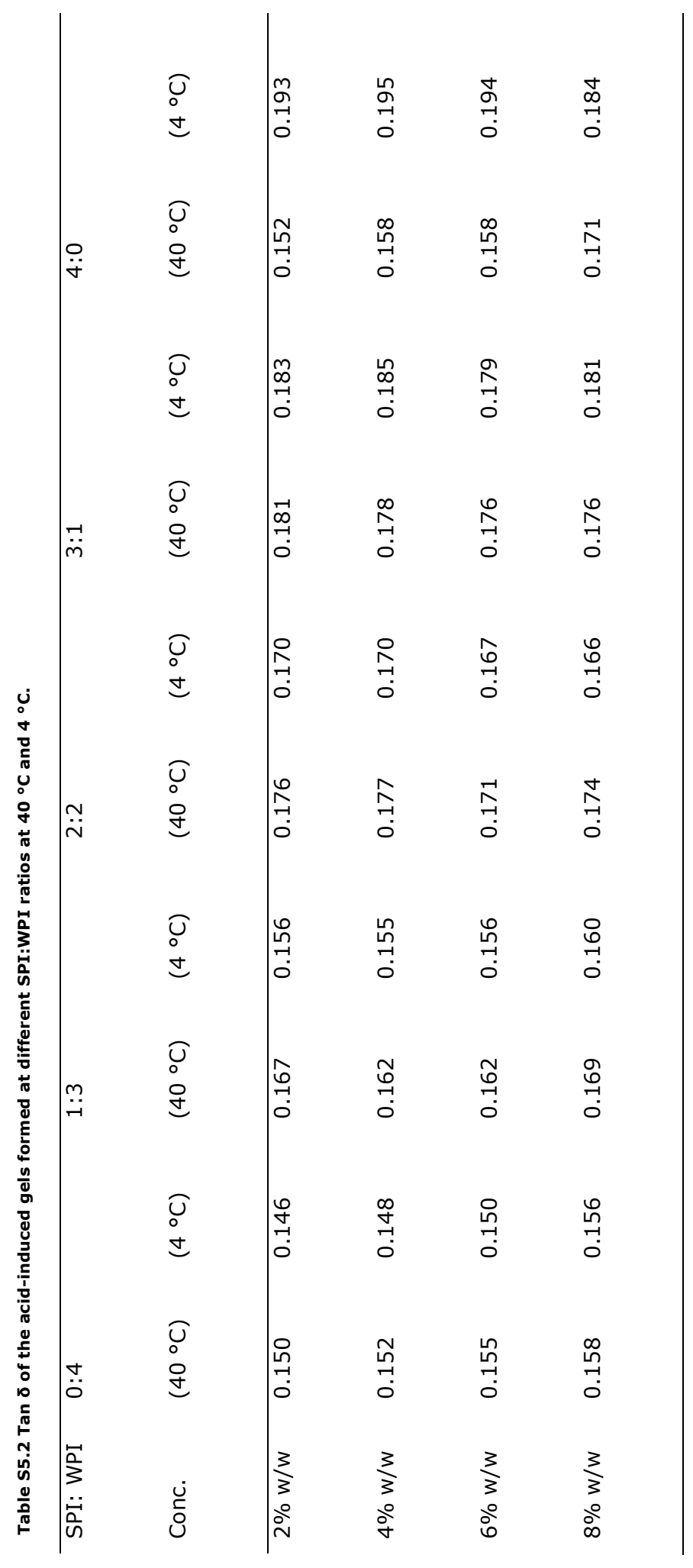





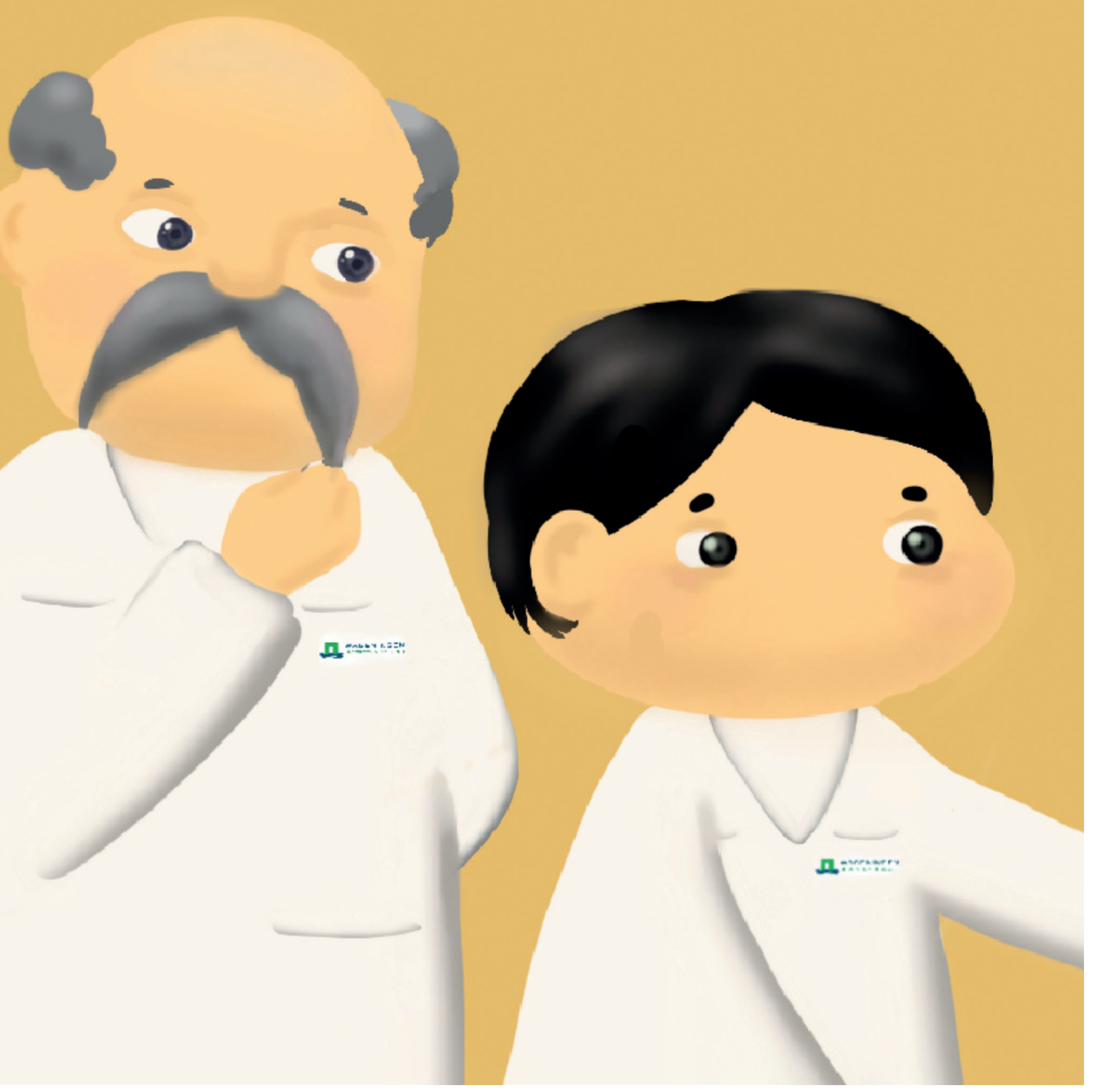




\section{Chapter 6}

\section{General discussion}




\subsection{Introduction}

In recent years, a transition from animal-based proteins to plant-based proteins has become a clear trend in both the food industry and food science field, driven by the demands for food security and sustainable development. Compared to the production of animal-based proteins, the production of plant-based proteins is a more efficient and economical way to convert nitrogenous matter to digestible protein for human consumption, since the latter requires fewer resources such as dedicated land and water. Soy protein is one of these plantbased proteins and has been focused on in this thesis. Although soy protein-based foods such as tofu have already been consumed in Asia for thousands of years, the technofunctional properties of soy protein are generally not comparable to animal-based proteins, which limits their application as a protein ingredient in the food industry. Native soy proteins have relatively large molecular weight, low molecular flexibility, and poor solubility, leading to a poor performance in interface-dominated systems such as emulsions and foams. Creaming could be observed in oil/water emulsions stabilized by soy protein after 60 days of storage, while emulsions stabilized by sodium caseinate, a commercial surfactant, were still stable [98]. Similarly, foam produced with soy protein decomposed more quickly with aging time than foam produced with whey protein, and the foam stability of the latter decreased significantly when soy protein was added, even at small soy-whey ratios [217]. Soy protein generally contains less Sulphur amino acids compared to animal-based proteins [218]. The production of soy protein often involves harsh extraction and purification processes, which causes the denaturation of protein and the formation of large insoluble aggregates. These could be detrimental to the gelling ability of soy proteins. For example, it has been found that heat-induced gels formed by soy protein have lower gel strength compared to gels formed by whey protein [219][220][193], micellar caseins [221], and egg protein [222].

The aggregation and gelation properties are very important for the application of soy proteins. To date, many efforts and attempts have been made to modify the properties of soy protein gels as well as the food products that are based on them. Figure 6.1 shows an overview of the research articles related to this topic as recorded by the Web of Science Core Collection Database from the year 2011 to 2020. Most of these studies (64\%) applied physical modifications, while $13 \%$ of them focused on enzymatic modifications or combined methods. Since the usage of chemical reagents in food products normally reduces the acceptance of consumers, only $10 \%$ of the articles adopted chemical approaches for soy protein modifications. Among the articles using physical modifications, most of the studies tried to mix soy protein with other food polymers such as proteins (27\%) and polysaccharides (43\%) directly, since, in real food production, the aggregation and gelation of soy proteins often occur in the presence of other food polymers, and therefore, the results 
of these studies could be more ready-to-use for application. As an eco-friendly and nonthermal physical modification, high-intensity ultrasound (HIU) treatment also has become a popular technique to modify the gelling ability of soy protein, which accounts for $8 \%$ of research articles.
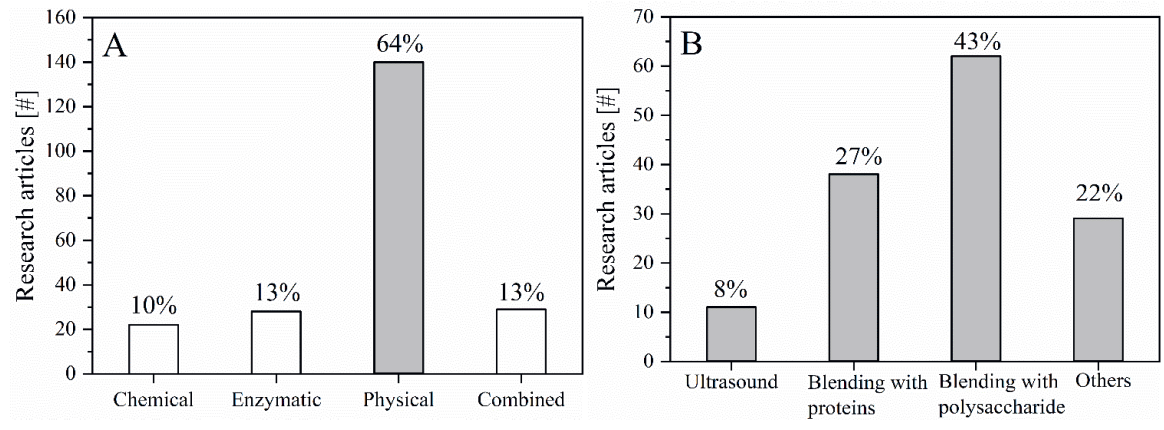

Figure 6.1. An overview of research articles on the most common modification techniques of soy protein gels and gel-related products (A), and specifically, the physical modifications (B), according to the Web of Science Core Collection Database from the year 2011 to 2020.

In this thesis, we investigated the aggregation and gelation properties of soy protein after several types of modification, such as selective proteolysis (Chapter $\mathbf{2}$ and 4), selective proteolysis combined with HIU (Chapter 3), and blending with whey proteins (Chapter 5). In this chapter, we will discuss the main findings of this thesis (as shown in Fig. 6.2) and compare the effects of the different modification approaches, and the mechanisms behind the changes they induce. As the application of a modified gel system in a real product needs a comprehensive understanding of its rheological properties, and the latter are closely related to the gel microstructure, we also extensively discuss the relationship between the rheological properties of gels and their microstructures, based on the results of this thesis and available literature. Finally, based on a reflection on the results of this thesis, we will provide some suggestions for further studies in this field. 


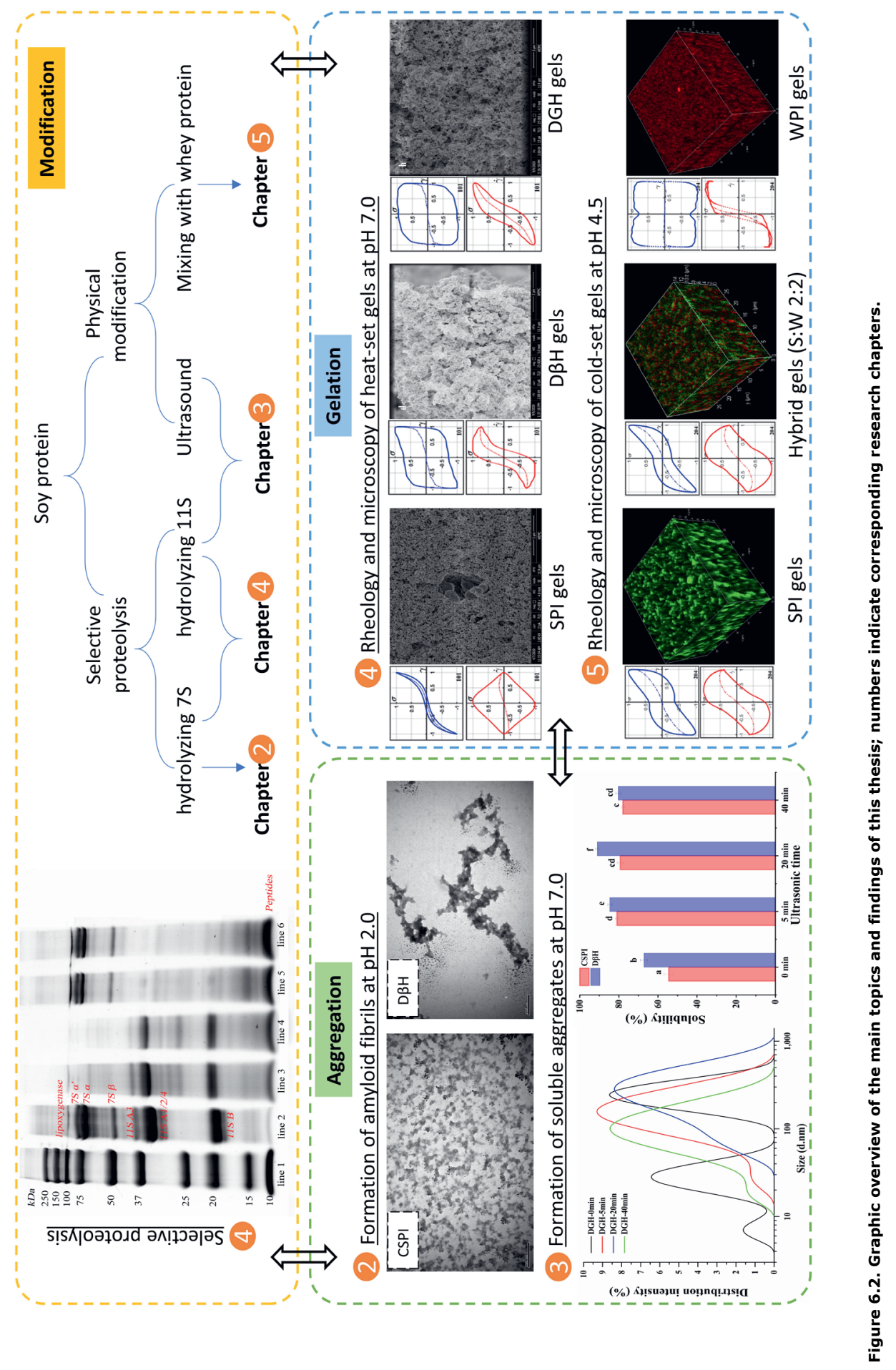




\subsection{How to modify the aggregation and gelation behavior of soy proteins?}

\subsubsection{Selective proteolysis}

Soy protein is a mixture of various protein components, with more than $80 \%$ of them being $\beta$-conglycinin and glycinin [33]. $\beta$-conglycinin and glycinin are significantly different in many aspects such as amino acid compositions, molecular structures, intramolecular forces, and denaturation behaviors. Hence, changing the ratio of $\beta$-conglycinin to glycinin, in a controlled way, could tune the aggregation and gelation behaviors of soy protein directly. Apart from the genetic modification of soy, this is commonly done by first extracting and separating these two components from native SPI, and then recombining them at different ratios. However, it is not economical to use this recombined "soy protein" in the food industry since the fractionation of soy protein components is a complicated and tedious process [193]. In this thesis, we have applied a method called selective proteolysis (Chapter 2, 3 and 4) to specifically hydrolyze the $\beta$-conglycinin or glycinin in soy protein isolate (SPI) without affecting the other proteins. Compared to the traditional enzymatic modification, this novel method allows us not only to hydrolyze the soy protein but also to vary the ratio of $\beta$ conglycinin to glycinin in the hydrolysate. By this method, we have obtained two types of hydrolysates, named degraded $\beta$-conglycinin hydrolysate (DßBH) and degraded glycinin hydrolysate (DGH), which proved to have significantly different protein compositions and functional properties.

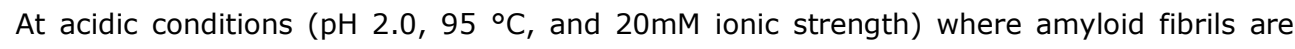
expected to form (Chapter 2), we found SPI and D 3 H both formed fibrillar aggregates as a function of time, but these amyloid fibrils displayed different morphologies. $D \beta H$ formed longer and semiflexible fibrils after heating for $60 \mathrm{~min}$ while the SPI formed short worm-like fibrils. After six-hour thermal treatment, $D \beta H$ fibrils further aggregated into larger clusters with a long-stranded shape, while the SPI fibrils formed small clusters with a small and more twisted conformation. Our results, for the first time, showed that amyloid fibrils can be formed by soy protein enzymatic hydrolysates, and selectively hydrolyzing $\beta$-conglycinin could contribute to the formation of long and semiflexible fibrillar aggregates. In line with our results, it has been found by others that the fibrils formed by purified glycinin were less branched than those formed by SPI [6]. In addition, isolated $\beta$-conglycinin was found to form worm-like fibrils with a twisted form, while isolated glycinin formed fibrils that were less flexible and associated into bundles [7]. Under the same conditions, their 1:1 mixture, however, formed irregular fibrils and much short-length debris [7]. These findings suggest that fibrillation processes of $\beta$-conglycinin and glycinin were different and could be mutually affected when these two components coexist in SPI. Apart from our study, only one study 
so far explored the effects of enzymatic proteolysis on the formation of soy protein fibrils [223]. In that study, the authors used traditional proteolysis and mentioned that the hydrolysate prepared by papain were less favorable for the formation of nanofibrils, but neither microscope images nor the enzymatic conditions were provided [223]. In here, the ability of $D \beta H$ to form longer and semiflexible fibrils could make it a promising thickening agent or structural ingredients, as these protein fibrils proved to have high viscosity and shear thinning behavior [6].

At neutral $\mathrm{pH}$, we systematically compared the thermal gelation behaviors of $\mathrm{D} \beta \mathrm{H}, \mathrm{DGH}$, and SPI (Chapter 4). To distinguish between effects which were introduced by changing the globular protein composition and those resulting from the generation of peptides, we first prepared each type of hydrolysate at two different degrees of hydrolysis (DH) by using the $\mathrm{pH}$-stat technique, and then divided each sample into a dialyzed one and a non-dialyzed one. As a result, eight hydrolysate samples were obtained (as shown in Fig. 4.1) and compared with SPI. It was found that no matter which component of soy protein was hydrolyzed, extensive hydrolysis was not favorable for the formation of a stiff gel. This could be attributed to the increased amount of small peptides ( $\leq 10 \mathrm{kDa})$, which may not be incorporated in the gel network [159]. This point was also supported by the observation that dialyzed hydrolysates (cut-off 12-14 kDa) formed stiffer gels than their non-dialyzed counterparts.

On the other hand, when the DH was controlled, we found that selectively hydrolyzing $\beta$ conglycinin rather than glycinin, could be beneficial to the gel stiffness. D $\beta H$ samples, especially the dialyzed one, could form significantly stiffer gels than SPI, while none of the DGH samples could. The improved gelation properties of plant-based proteins after traditional proteolysis has also been reported $[133,224,225]$. The enhanced strength of $D \beta H$ samples could be attributed to two aspects: 1 ) like in traditional proteolysis, the enzymatic cleavage of $\beta$-conglycinin unfolded the original protein structure, which exposed ionizable groups and hydrophobic groups to the surface, thus contributing to the interaction between proteins; 2 ) selectively hydrolyzing $\beta$-conglycinin and removing the peptides, significantly increased the relative content of glycinin. Glycinin is known to form stiffer gels than $\beta$ conglycinin since the former contains more sulfhydryl groups and is thus able to form more disulfide bonds to maintain a strong-linked gel structure. The importance of disulfide bonds in maintaining the gel structure was also shown when we compared the acid-induced soy protein gels with whey protein gels (Chapter $\mathbf{5}$ ).

Selective proteolysis can also be beneficial when a lower gelling temperature is desired. Both $\beta$-conglycinin and glycinin in SPI were denatured to some extent during the proteolysis, which led to an earlier onset of gelation. This shortening of gelation onset was more obvious 
when glycinin was hydrolyzed since glycinin is the component that has a higher thermal stability and denaturation temperature. The $D \beta H$ samples with limited $\mathrm{DH}$ could be a promising gelling ingredient for meat products such as sausages, where the protein additives are required to form strong gel networks at lower temperatures. Our results also provide first evidence that hydrolyzing different components of soy protein, even at the same $\mathrm{DH}$, could produce heat-induced gels with distinct microstructures and nonlinear rheological behaviors, which we will discuss in Section 6.3.

For other functional purposes, selective proteolysis also has proved to be more efficient than traditional proteolysis. For instance, when it comes to emulsion properties, selectively hydrolyzing glycinin instead of $\beta$-conglycinin can be preferential, since glycinin is known to have higher molecular weight and a more compact structure which are not favorable for the emulsifying activity and foaming ability $[33,226]$. This point has been verified by Li et al. who found that DGH (which they called reduced glycinin hydrolysate, RG) was a better emulsifier compared to isolated $\beta$-conglycinin and the hydrolysates prepared by traditional proteolysis, and could be used as a substitute for sodium caseinate (a widely used commercial emulsifier). Another example of the benefits of selective proteolysis was shown by Margatan et al.: enlightened by the fact that $\beta$-conglycinin showed greater potential than glycinin for reducing the risk of cardiovascular diseases, they applied selective proteolysis and found that $\mathrm{D} \beta \mathrm{H}$, even with lower $\mathrm{DH}$, had a higher content of antihypertensive peptides than DGH [39]. Therefore, selective proteolysis allows one to produce hydrolysates with high angiotensin-converting enzyme (ACE) inhibitory activity without performing extensive hydrolysis and unnecessary fractionation [39]. 


\subsubsection{Physical modification and its combination with proteolysis}

Unlike selective proteolysis that can break peptide bonds and change the protein composition, physical modifications normally affect the conformational structures of proteins instead of their primary structures. High-intensity ultrasound (HIU) is such a physical method that has been widely used in protein modification. Owing to the cavitation phenomenon, HIU can induce changes in a protein system in several ways: 1) disrupting the inter- or intramolecular interactions of macroaggregates; 2) unfolding (partially) the native structures of proteins and exposing their buried hydrophobic groups and sulfhydryl groups; 3 ) inducing the re-association and aggregation between small and unfolded protein particles, by enhanced particle collision and oxidation-induced/thermally-induced interactions. Although these different aspects could cause different results in terms of the physicochemical properties, they are generally beneficial to the gelation properties of soy protein, as shown in Table 6.1. For example, Tang et al. found HIU could facilitate the transformation of insoluble aggregates to soluble aggregates in soy protein dispersions, which improved the elasticity of heat-induced gels [106]. Similarly, Hu and colleagues have found HIU-treated soy protein could form better cold-set gels with higher strength, water holding capacity, and finer gel structures compared to unmodified soy protein, no matter the coagulant was salt [227], acid [95,228], or enzyme $[229,230]$.

Like the $\mathrm{DH}$ in proteolysis modification, the extent of HIU treatment (here the interplay of intensity and time) decides the final effects of this modification (Table 6.1), especially in terms of protein aggregation. For instance, at the beginning of HIU treatment, the dominant effect might be the localized shear force generated by cavitation, which reduces the size of protein particles, especially for those macroaggregates that are originally present in the sample [228]. As the HIU treatment is prolonged, the protein structure gradually unfolds and exposes reactive groups. Proteins in these states are more likely to interact with each other and form aggregates, especially at an increased rate of collision $[106,228]$. Similarly, Jiang et al. found that low-power HIU $(72-78 \mathrm{~W} / \mathrm{cm} 2)$ disrupted the internal hydrophobic interactions of protein molecules and accelerated protein molecular motion, leading to protein aggregation; medium-power HIU (96-104 W/cm2) broke up unstable aggregates; high-power HIU (112-120 W/cm2) induced repolymerization of small aggregates through noncovalent interactions. 


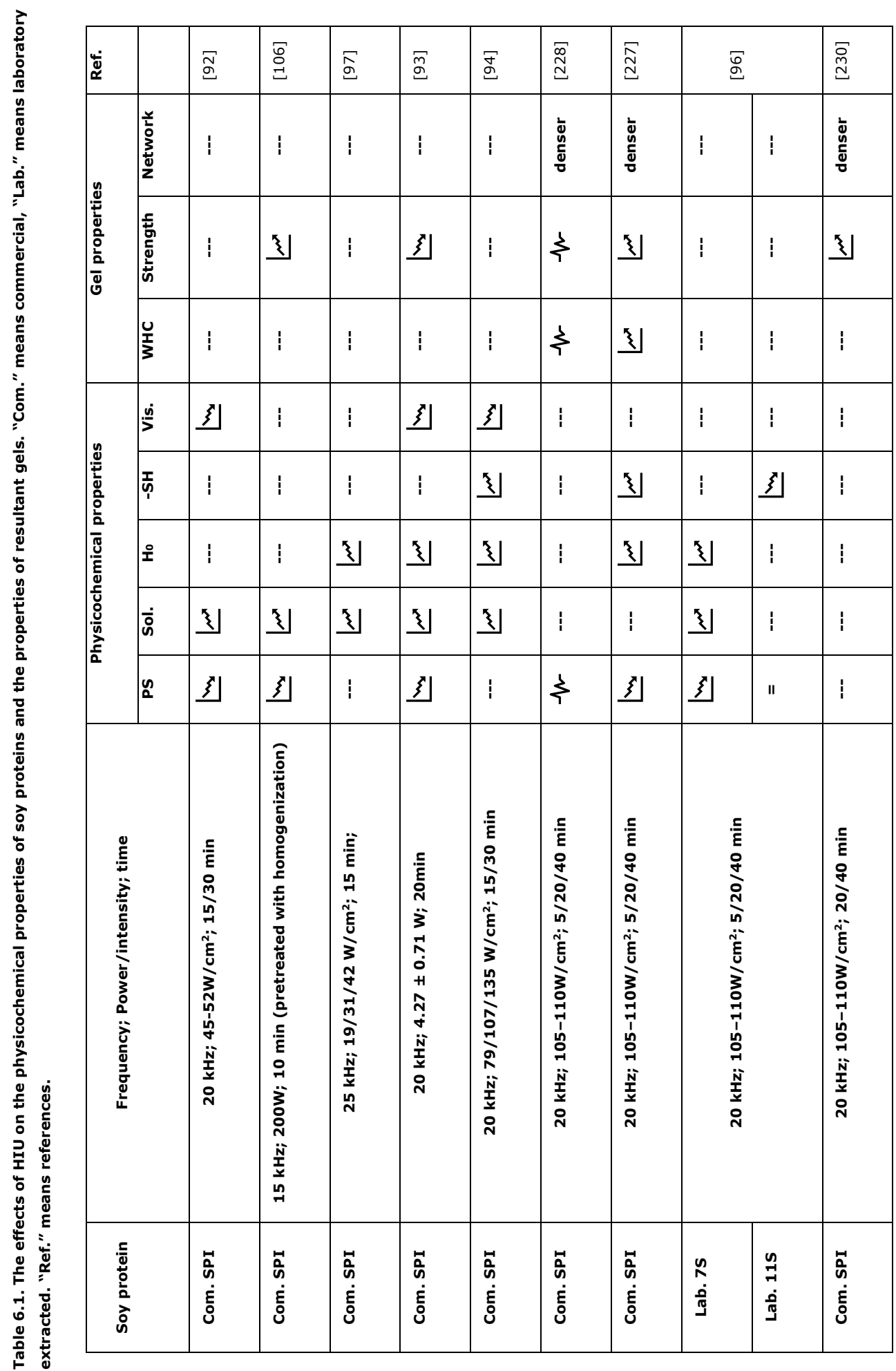




\begin{tabular}{|c|c|c|c|c|c|c|c|}
\hline 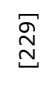 & 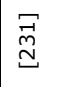 & $\bar{\sigma}$ & $\underset{\underset{N}{\widetilde{V}}}{\bar{N}}$ & 呑 & 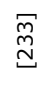 & 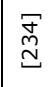 & \begin{tabular}{|l}
$\overline{\mathscr{N}}$ \\
\end{tabular} \\
\hline 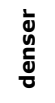 & 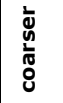 & i & 1 & 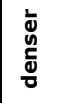 & 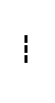 & 1 & : \\
\hline 立 & 3 & i & $\xi$ & $\xi$ & 1 & $\xi$ & 3 \\
\hline ㅊ & & : & I & $\xi$ & I & 3 & : \\
\hline : & & 1 & $\xi$ & & I & 1 & 1 \\
\hline : & $"$ & i & 1 & & $\xi$ & 1 & 1 \\
\hline : & $\xi$ & $\xi$ & 1 & 3 & 치 & $\xi$ & : \\
\hline 1 & & 3 & $\xi$ & $\xi$ & ! & 1 & $\xi$ \\
\hline | & & $\xi$ & i & $\xi$ & 文 & $\xi$ & 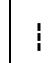 \\
\hline 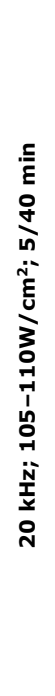 & 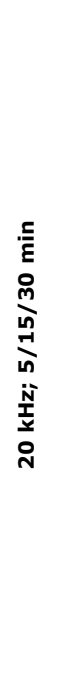 & 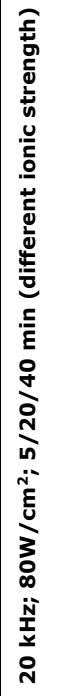 & 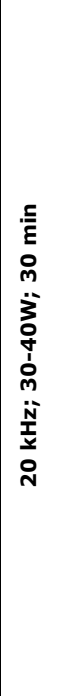 & 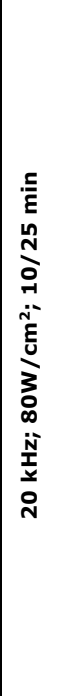 & 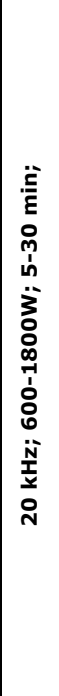 & 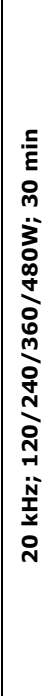 & 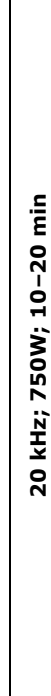 \\
\hline 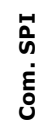 & 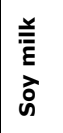 & 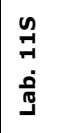 & 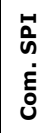 & $\begin{array}{l}\overrightarrow{0} \\
\hat{\omega} \\
\dot{0} \\
\tilde{\omega}\end{array}$ & 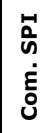 & \begin{tabular}{|l}
$\overrightarrow{0}$ \\
$\hat{\omega}$ \\
$\dot{\omega}$ \\
$\tilde{\omega}$
\end{tabular} & $\begin{array}{l}0 \\
\ddot{0} \\
\dot{\varepsilon} \\
\dot{\Xi}\end{array}$ \\
\hline
\end{tabular}

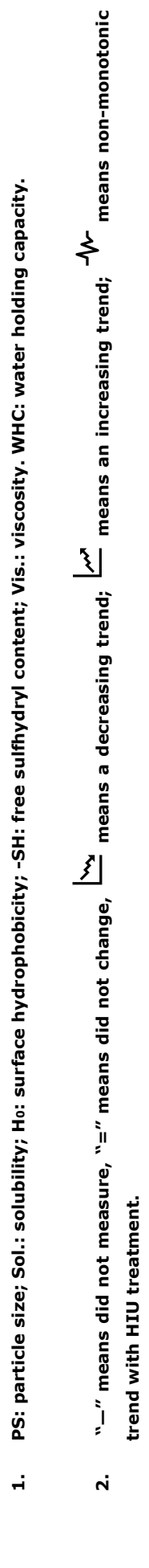


The extent of HIU modification has also been found to depend on the composition and aggregation state of proteins. HIU showed significant effects on commercial SPI/SPC $[94,235]$, native SPI [95], and isolated $\beta$-conglycinin [96], but seemed to have no or minor effects on isolated glycinin, because of its rigid structure and highly aggregated state. For this reason, in Chapter 3, we applied selective proteolysis to first hydrolyze the glycinin and then subjected DGH to a HIU treatment $\left(20 \mathrm{kHz}, 34-37 \mathrm{~W} / \mathrm{cm}^{2}, 5 / 20 / 40 \mathrm{~min}\right)$, with the aim to explore the combined effects of proteolysis and HIU modifications. Our results showed that the effects of HIU on the physical, structural, and aggregation properties of DGH and non-hydrolyzed SPI both depend on the treatment time. Within a certain duration, HIU treatment induced the formation of soluble aggregates and increased the solubility of samples, but these soluble aggregates were unstable and could be dissociated again under prolonged sonication. Pretreating SPI by selectively hydrolyzing the glycinin component contributed to the HIU-induced formation of soluble aggregates because the enzymatic cleavage disrupted the original macroaggregates and unfolded the rigid structure of glycinin, exposing more reaction sites for the subsequent aggregation. Furthermore, the increased ratio of $\beta$-conglycinin: glycinin could also be beneficial to the formation of soluble aggregates as Guo et al. found that $\beta$-conglycinin tended to form soluble aggregates while glycinin formed insoluble aggregates during thermal treatment. Overall, under the same time of HIU treatment, DGH displayed higher solubility and better gelling ability than non-hydrolyzed SPI, showing the synergistic effects of selective proteolysis and HIU modification.

Apart from our study, there is one study by Tian et al. which also treated soy protein hydrolysates with HIU [236]. In that study, soy protein hydrolysates with different DH (not provided) were prepared by traditional proteolysis with Alcalase, and HIU treatment also proved to increase the solubility of these hydrolysates as well as the antioxidant capacity. However, the authors attributed the improved solubility to the degradation of peptide aggregates. These divergent mechanisms, again, cement the point that the effects of HIU treatment depend on the aggregation state of the starting material, since the hydrolysates in the study of Tian et al [236] contained a large number of insoluble aggregates and even two separated phases, while these phenomena were not observed in our DGH samples. For the reversed order, so HIU pretreatment and subsequent proteolysis, synergistic effects have been reported more often. It has been found that HIU pretreatment could benefit the proteolysis of soy protein by improving the accessibility of protein subunits to the enzyme such as papain [97], Alcalase [237], or bromelain [233]. The hydrolysates from HIUpretreated soy protein showed higher solubility, emulsifying ability, and antioxidant capacity than the hydrolysates from untreated SPI $[97,233,237]$. 


\subsubsection{Blending soy protein with animal-based proteins}

Blending plant-based proteins with animal-based proteins becomes attractive in the formulation of food products due to advantages in the economic, nutritional, environmental, and techno-functional aspects [238]. To this end, it is important to understand the aggregation and gelation properties of soy protein in the admixture of animal-based proteins. Table 6.2 summarizes the studies on the mixed systems of soy protein and animalbased proteins, with the focus on the aggregation and gelation properties. As shown, most of the studies investigated heat-induced aggregates or hybrid gels, while few of them focused on cold-set hybrid gels. The research group of Corredig has first explored the GDLinduced gelation of the heated mixture of commercial SPC and commercial skim milk powder [194]. At the same solid content $(12 \% \mathrm{w} / \mathrm{w})$, replacing commercial skim milk powder with commercial SPC, up to a ratio of 1: 1, shortened the onset of gelation and strengthened the gels, while further replacement did not affect the gelation anymore [194]. The microstructure of these hybrid gels did not show phase separation, although larger aggregates were observed in the gel network when the amount of SPC was higher [194]. They further explored the effects of soluble soy protein (SSP) on the gelation of casein micelles with/ without whey protein and found that the heated mixture of SSP and casein micelles showed an earlier onset of gelation and a higher $\mathrm{G}^{\prime}$ value compared to casein micelles alone [188]. However, when mixing SSP with casein micelles and whey protein, the changes in the gelation behavior seemed to be dominated by whey protein, while the effects of soy protein were negligible [188]. The authors attributed these discrepancies to the different composition and processing history of soy proteins [188]. In fact, the starting materials in previous studies were mainly commercial SPC, soy milk, reconstituted and fresh milk [188,194,239-241], which were rather complicated and impure protein systems even before blending, making it difficult to ascertain the gelling behavior of soy protein in the admixture of a specific dairy protein. 


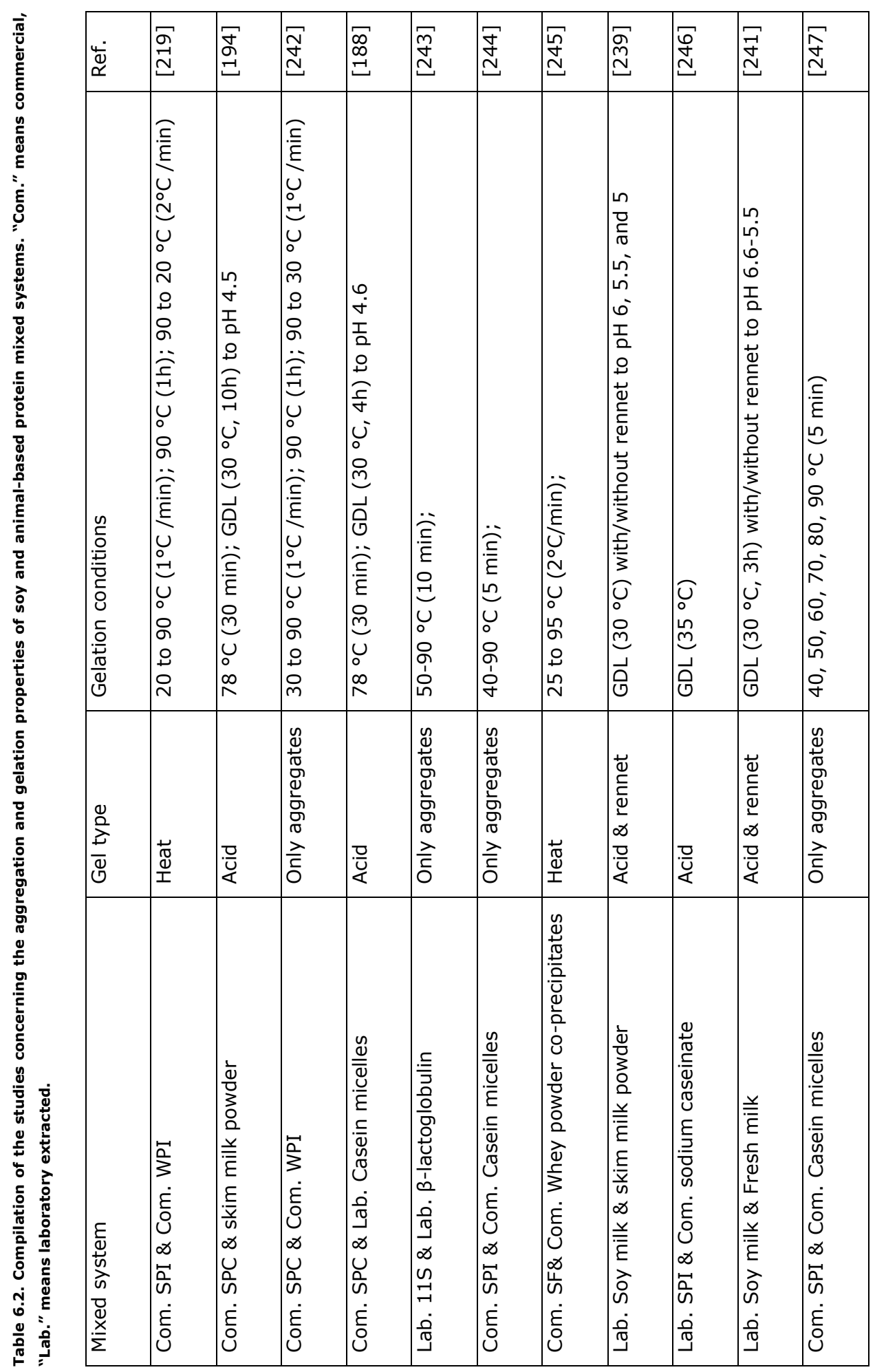




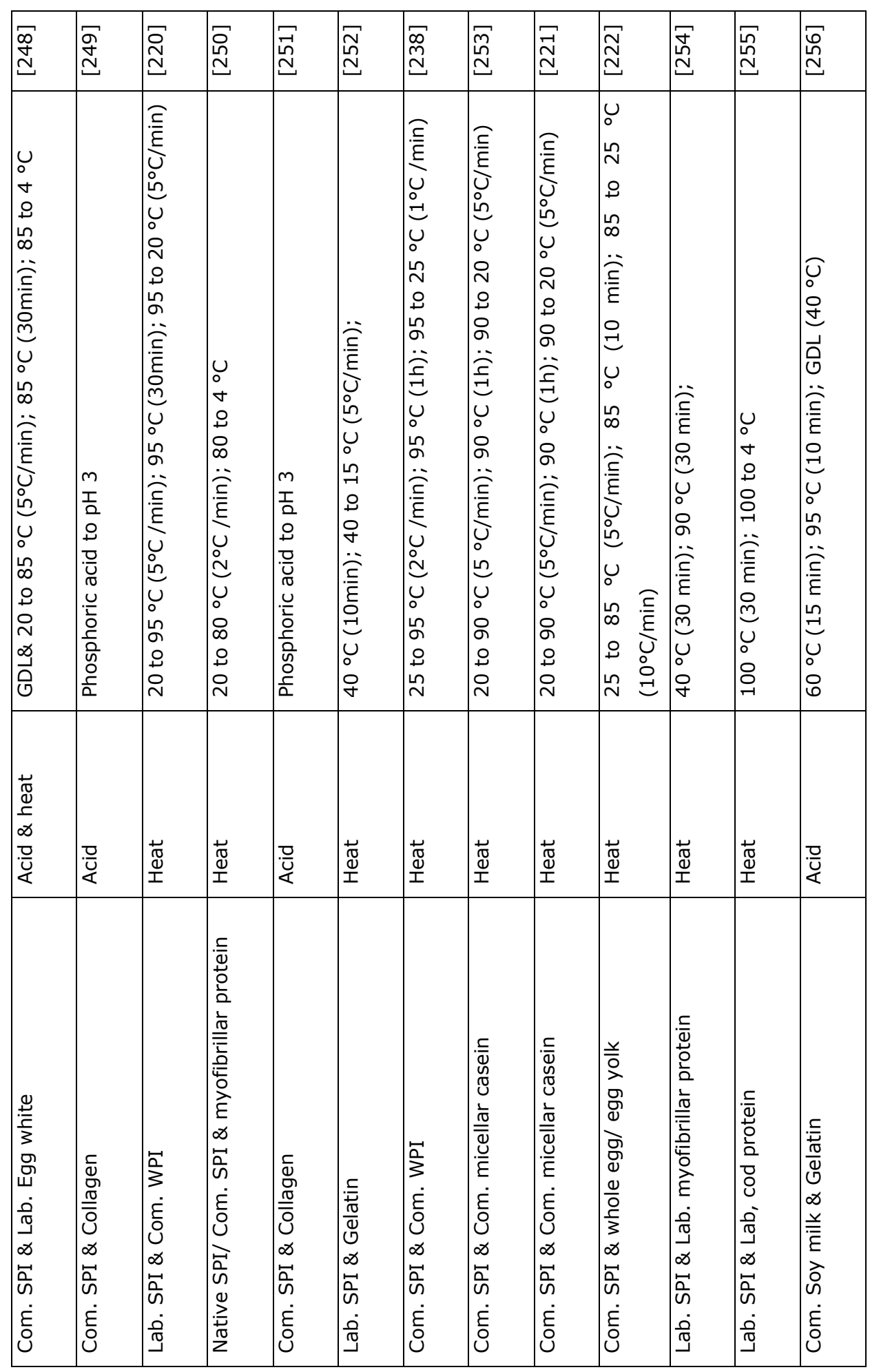


Taking the above into consideration, commercial SPI with commercial WPI were used in Chapter 5, both of which had protein content higher than $90 \%$. We blended them at different ratios and protein concentrations, to explore the thermal aggregation and acidinduced gelation of soy and whey protein mixtures. We found that more insoluble aggregates and fewer soluble aggregates were formed in the mixture during heating ( $95^{\circ} \mathrm{C}, 30 \mathrm{~min}$ ) when the SPI proportion was higher. Similarly, Anuradha et al. found that the mixture of $\beta$ lactoglobulin and glycinin showed higher turbidity and lower solubility after heating $\left(90^{\circ} \mathrm{C}\right.$, $10 \mathrm{~min}$ ) when the proportion of glycinin was higher. Our results also indicated that the strength of the electrostatic repulsion between protein aggregates could be similar in all heated mixtures as their zeta-potential were similar (around $-28 \mathrm{mV}$ ), at the same $\mathrm{pH}$ and ionic strength. The surface hydrophobicity of the heated mixture was higher when the proportion of SPI was higher, indicating the aggregates in these mixtures had a higher tendency for hydrophobic interaction. We also found that the secondary structures of mixtures were dependent on the ratio of SPI: WPI before heating: the a-helix and $\beta$-sheet structures proportionally decreased with SPI content while the random coil and $\beta$-turn structures increased. Interestingly, these trends were not observed after heating, and the secondary structures of soluble aggregates seemed to be similar and independent of the protein composition. (Fig. 6.3). To our knowledge, these are the first findings on the secondary structures of soy and whey protein mixed aggregates.
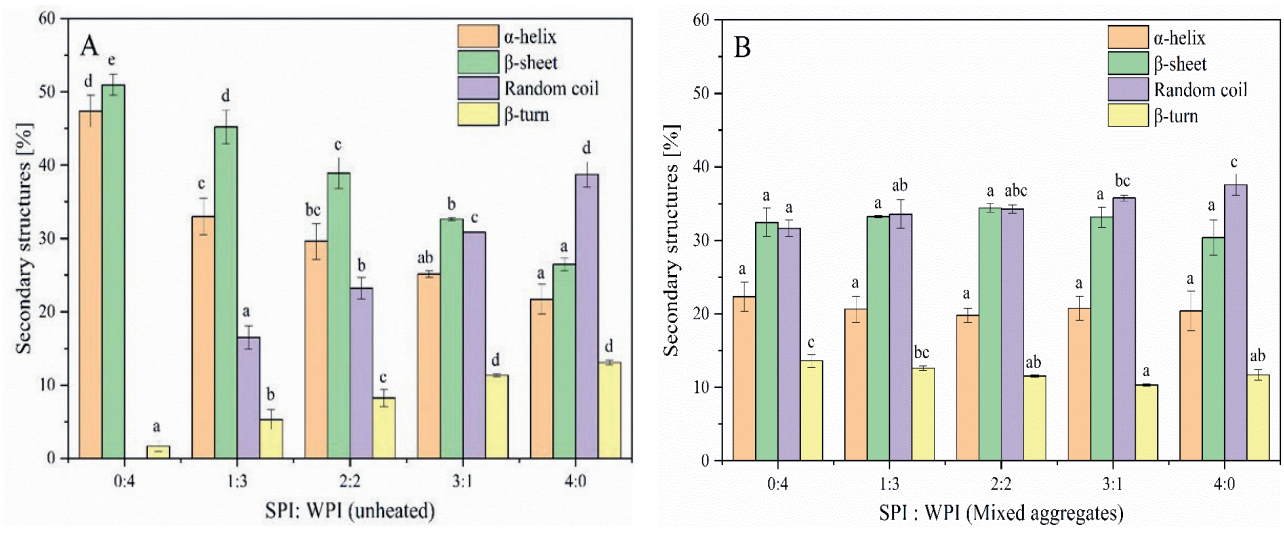

Figure 6.3. Secondary structure composition of soluble fractions of the soy protein and whey protein mixtures before heating (A) and after heating (B). 
Subsequently, we investigated the GDL-induced gelation of these mixed protein aggregates. It was found that the acidification rate and gelling rate of samples were decreased with increasing ratios of SPI: WPI, and the resultant gels had lower strength (lower $\mathrm{G}^{\prime}$ ), stretchability (lower $Y_{c}$ ), and water holding capacity. Furthermore, we found that the rheological behaviors of the hybrid gels in the non-linear viscoelastic (NLVE) regime were more similar to SPI gels than to WPI gels. Compared to WPI gels, the hybrid gels and SPI gels displayed a relatively more elastic response in the NLVE regime and a more gradual transition to the plastic behavior, which could be explained by the different microstructures and driving forces as we will discuss later on. Previous studies reported that soy protein had little impact on the rheological properties of heat-induced hybrid gels formed by soy and whey protein mixtures [238]. The formation of heat-induced gel networks was dominated by whey proteins, and the gel networks were not influenced by the distribution of soy proteins [238]. When the total protein content was fixed, replacing WPI with SPI led to lower gel strength but higher WHC and fracture strain [220]. These different findings show that the acid-induced gelation of soy and whey protein mixtures is significantly different from their heat-induced gelation. Our results will contribute to the understanding of the partial replacement of whey protein with soy protein in acid-induced gel matrices.

\subsection{Linking rheological properties with gel microstructure}

Although it has been generally accepted that the rheological properties of gel matrices are dependent on their microstructures, a clear relationship between these two is difficult to established. To contribute to this area, this thesis explored in detail how the linear and nonlinear rheological properties of soy protein heat-induced gels (Chapter 4) and acid-induced gels (Chapter 5) changed after protein modifications like selective proteolysis (Chapter 4 ) and blending with whey proteins (Chapter 5). We used various microscopy techniques to visualize the gel microstructures and applied fractal scaling theory to interpret the different rheological properties from the microstructural perspective.

The rheological properties of soy protein gels are related to the density of gel networks. As shown by SAOS tests, the $\mathrm{G}^{\prime}$ of all gels in this thesis increased with protein concentrations. This indicated that soy protein gels were stiffer when more proteins participated in the gelation and formed a denser microstructure. However, the density of the microstructure is not the only factor determining gel strength. As shown in Fig. 6.4, heat-induced soy protein gels formed at $10 \% \mathrm{w} / \mathrm{w}$ protein concentration showed a much denser microstructure than the acid-induced gels formed at $4 \% \mathrm{w} / \mathrm{w}$, although these two gels displayed similar $\mathrm{G}^{\prime}$ (around $1000 \mathrm{~Pa}$ ) in the SAOS tests. To understand this difference, other factors need to be considered, e.g. the type of gel network which is formed. It can be seen that soy proteins formed a coarse particulate or aggregated gel network after heat-induced gelation, while 
they formed a more fine-stranded gel network in acid-induced gelation. Using the fractal scaling model of $\mathrm{Wu} \&$ Morbidelli, we found these two gels both belong to the intermediate gel type, in which both inter-floc and intra-floc links contribute to the overall gel elasticity. Despite this, heat-induced gels were relatively closer to weak-link type gels compared to acid-induced gels, as the $a$ value of the former was higher than the latter $(0.75$ versus 0.32$)$ and comparatively closer to one (the value for an ideal weak-link system). So, here the observation that a dense particulate gel network formed by heat-induced gelation did not exhibit a higher $\mathrm{G}^{\prime}$ than a fine-stranded gel network form by acid-induced gelation, could be attributed to the weaker inter-floc links in the former.
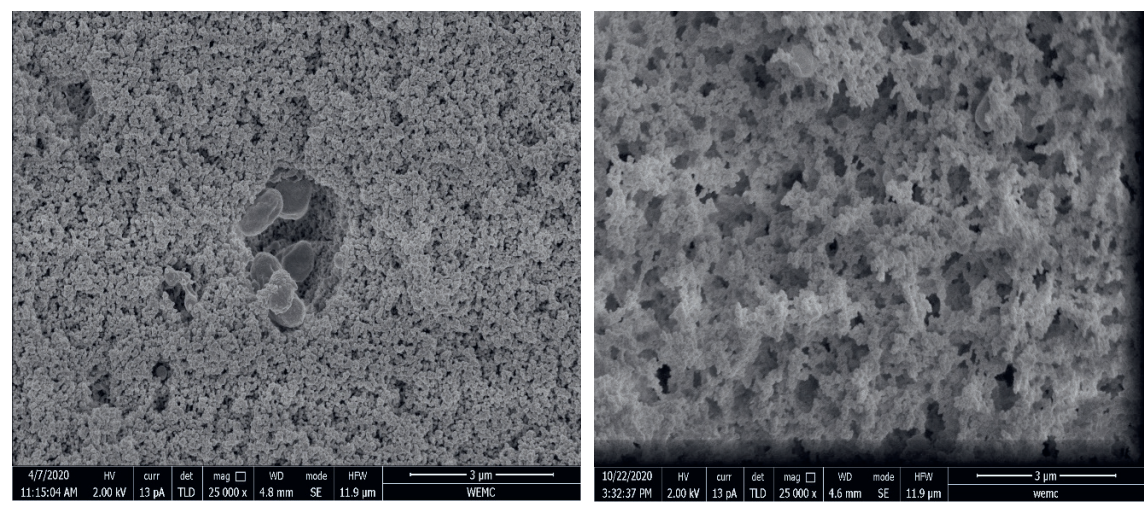

Figure 6.4. SEM images (magnification $25,000 \times$ ) of $10 \% \mathrm{w} / \mathrm{w}$ heat-induced soy protein gels (left) and $4 \% \mathrm{w} / \mathrm{w}$ acid-induced soy protein gels (right).

At the same protein concentration and using the same gelation process, we found selective proteolysis significantly changed the microstructure of soy protein gels as well as their rheological properties (Chapter 4). Through CLSM images, we found D $\beta H$ formed a denser and more homogenous gel microstructure than native SPI, which well explained their higher $G^{\prime}$ in the linear viscoelastic (LVE) regime. In general, the $G^{\prime}$ of protein gels increase with the homogeneity of their gel networks, since the finer gel structures have a higher water holding capacity, and when more water is held inside the gel, less energy will be lost via dissipation [257]. With SEM, we found that although DBH gels and SPI gels both belonged to the class of particulate gel network, the constituent flocs in the former were more compact and consisted of larger protein particles. The higher rigidity of these compact flocs could contribute to the stiffness of $D \beta H$ gels, but also made $D \beta H$ gels much more brittle, as verified by their lower $\gamma_{c}$ during LAOS tests. The microstructure of DGH gels was much more heterogeneous, and contained larger pores and cracks. Under an applied stress, DGH gels could be easily disrupted as a result of these pores and cracks which were probably the weakest elements of the gel network [258]. As a result, DGH gels displayed the lowest G' and a clear syneresis. With SEM, we found that the constituted flocs in DGH gels appeared 
to be smaller and more branched. Although these flocs may have resulted in a lower stiffness, they were more entangled and intertwined with each other, and as a result DGH gels were classified in the strong-link regime. The inter-floc links in DGH gels manifested in a weak strain overshoot (Type III) behavior and a gradual yielding behavior at large deformation.

Acid-induced gels formed by WPI showed a homogenous and fine-stranded gel network, and the constituent flocs were closely packed in 3D space. These structural features made the WPI gels very stiff and stretchable, showing the highest $\mathrm{G}^{\prime}$ and $\gamma_{c}$ among all the acid-induced gels. Replacing WPI with SPI significantly coarsened the gel microstructure, and more gaps and pores were observed with increased SPI content. In these gels that contained SPI, the constituent flocs became larger and had more elongated and irregularly branched structures. Again, using the model of Wu \& Morbidelli, we found that the inter-floc links in WPI gels were very strong and dominating the behavior, as the $a$ value (0.03) was almost equal to zero, while the gels that contained SPI were all categorized as intermediate type gels. Due to the coarse and more weakly-interconnected network, the gels containing SPI could not resist high stress and strain, which was manifested by a low $\mathrm{G}^{\prime}$ and $\gamma_{c}$. Meanwhile, more branched structures would increase the chance of these flocs to entangle with each other under shear flow, and to rejoin the network. As a result, the gels that contained SPI exhibited a much slower transition to plastic behavior during LAOS tests and retained some elasticity even at the highest strain amplitude we studied $\left(y_{0}=1000 \%\right)$. Overall, the results in this thesis demonstrated that the rheological properties of soy protein heat-induced gels and acid-induced gels can be modified by selective proteolysis and blending with whey proteins. The different rheological properties, especially in NLVE regime, can be well correlated with the microstructural features, including the density, homogeneity, and type of gel networks, as well as the conformation and interactions of constituent flocs.

\subsection{Reflections and recommendations}

The protein transition from animal-based to plant-based resources can be expected to continue in the future. Motivated by this trend, this thesis took soy protein, a benchmark of plant-based proteins, as the subject, and investigated their aggregation and gelation properties after several enzymatic and physical modifications, such as selective proteolysis and high-intensity ultrasound. In general, all these approaches successfully changed the aggregation and gelation properties of soy protein, by altering their particle sizes, conformational structures, protein compositions, and molecular interactions. Which method to choose, depends largely on the application one is considering, and on several other factors. 
To begin with, the source of the soy protein should be understood thoroughly before a choice is made for a particular modification method. In the literature, different studies often started with different soy protein materials. These protein materials could have different protein purities (e.g. soy flour and soy protein isolate), protein compositions (e.g. isolated 7S and isolated 11S), and denaturation state (e.g. commercial SPI and lab-extracted SPI). As a result, the interstudy comparison between different modification methods is almost impossible, and divergent results are often found in the soy protein research field, even from the same research group. To solve this problem, a more detailed characterization of the starting material should be advocated as a standard requirement for future studies. Just like most of the current studies measured the protein content of soy protein materials, future studies should also check the denaturation state of the starting material via differential scanning calorimetry (DSC) or the ratio of $\beta$-conglycinin to glycinin via SDS-PAGE.

During soy protein modification, one should also realize that the final effects often depend on the extent of treatment, and the latter is decided by the operating parameters. For example, when applying enzymatic proteolysis, the final effects depend on the degree of hydrolysis $(\mathrm{DH})$, and this $\mathrm{DH}$ is decided by operating parameters such as time, $\mathrm{pH}$, temperature, and enzymatic activity. Within a certain limit, increasing the $\mathrm{DH}$ will contribute to the unfolding of soy protein structures, and thus benefit the aggregation and gelation. When the $\mathrm{DH}$ is too high, soy protein could be broken down into small peptides, which lose the reactive groups and their ability to form gel networks. Similarly, when applying highintensity ultrasound (HIU), the duration and intensity of ultrasonic treatment are important. Proteins could be partially unfolded and form soluble aggregates within a certain time and power range, while intensive treatment could disrupt the aggregates and trigger undesired protein oxidation. This kind of phenomenon can be also seen in other modification methods such as heating and high-pressure treatments. Therefore, for achieving the desired improvement and saving modification costs, future studies on soy protein modification should carefully consider the extent of treatment and explore the optimal processing parameters.

When the gelation properties are of interest, large amplitude oscillatory shear (LAOS) tests should be performed on a more regular basis in addition to small amplitude oscillatory shear (SAOS) tests. In this thesis, we successfully applied LAOS tests on both soy protein heatinduced gels and acid-induced gels, to reveal their rheological properties in the non-linear viscoelastic regime. Normalized Lissajous plots proved to be a very useful tool to analyze the LAOS data, since they visualize the non-linear viscoelastic properties in a more distinctive way than traditionally used plots of first harmonic moduli versus strain. Nevertheless, LAOS tests are still far from mainstream in food science, especially for protein gels. To establish the relationship between the non-linear viscoelastic properties and gel 
microstructures, more work is needed. To this end, the collaborations with other researchers from the engineering and material science fields would be helpful for food scientists to understand the theoretical underpinnings of LAOS tests, and thus to make the best use of this valuable tool in food materials.

Although many studies have realized that oral processing involves large stresses and strains, only two studies so far have explored the relationship between the sensory properties of food products with their nonlinear viscoelastic properties. By applying LAOS tests, descriptive sensory analysis, and oral processing recording, Melito et al. [141,142] demonstrated that the nonlinear viscoelastic properties of WPI/ $\mathrm{k}$-carrageenan gels and different commercial cheeses could be well correlated $\left(R^{2}>0.5, P<0.05\right)$ to their sensory perception. It was found that $G_{L}^{\prime} / G_{M}^{\prime}$ and $G_{3}^{\prime} / G_{1}^{\prime}$ were negatively correlated with compressibility (degree of sample deformation/compression before fracture using tongue and hard palate), while positively correlated with chalkiness (extent of perception of fine, chalk-like particles during mastication) and residual particles (number of particles remaining in mouth after expectorating a sample). In addition, nonlinear viscoelastic properties were also found to correlate with oral processing characteristics $(P<0.05)$ including jaw movement and muscle activity $[141,142]$. These results suggest that LAOS tests can be used to indicate how gel-based foods deform and break down in the mouth, as these soft solids are normally chewed by shear forces rather than compressive forces. However, whether these sensory correlations are universal and applicable to our LAOS data of soy protein gels still needs to be verified in the future. In conclusion, the present thesis not only expands the knowledge of soy protein aggregation and gelation, but also provides a research mode for other protein gels in terms of the interplay between protein modification, rheological properties, and microstructures, which will benefit the rational design of gelbased foods and accelerate the transition to plant-based proteins. 




\section{Summary}

The concept of sustainable development drives food manufacturers and consumers to reduce the consumption of animal-based proteins and switch to plant-based proteins. Soy protein is such a plant-based protein which has been used in traditional Asian cuisine for thousands of years. Soy protein contains well-balanced amino acids and physiologically beneficial components, which makes it a promising substitute for animal-based proteins. However, the functional properties, such as emulsification, gelation, foaming properties, of soy proteins are often not comparable to that of traditional animal-based proteins, which limits the application of soy proteins in food industry. Research efforts have therefore been made to improve the functional properties of soy proteins, and various protein modification methods have been studied. Aggregation and gelation properties are the most important properties of soy proteins, which have been applied in the production of many texturized foods, such as tofu, sausage, and meat analogues. In this thesis, we focused on the aggregation and gelation properties of soy proteins, and investigated how these properties were modified by several state of the art modification methods and the mechanisms behind these changes. As the application of a new gel system needs a comprehensive understanding of its rheological properties, and the latter are closely related to the microstructure of gels, we also aimed to gain deeper insight into the interplays between protein modification, rheology, and gel microstructures. In Chapter 1, as a basis to the aim of this thesis, we presented an overview on the composition, aggregation, and gelation of soy proteins. As the most common methods in protein modification research, we explain the principle of enzymatic and physical modifications, specifically selective proteolysis and ultrasound treatment. We also provided a detailed introduction on the rheological methods that were applied in this thesis, such as large amplitude oscillatory shear (LAOS) tests and fractal scaling theory.

In Chapter 2, a novel method called selective proteolysis was applied on soy protein isolates (SPI). By this method, we selectively and exclusively hydrolyzed the $\beta$-conglycinin component of soy protein while keeping glycinin unaffected, which resulted in a degraded $\beta$-conglycinin hydrolysate ( $\mathbf{D} \boldsymbol{\beta} \mathbf{H})$. Subsequently, we compared $D \beta H$ with a non-hydrolyzed control SPI (CSPI) sample, in terms of the aggregation behavior upon heating $\left(95^{\circ} \mathrm{C}\right)$ at $\mathrm{pH}$ 2.0 and low ionic strength $(20 \mathrm{mmol} / \mathrm{L})$. We were interested in these conditions since a special aggregation behavior of proteins, i.e. amyloid fibrillation, has been known to occur at these conditions. Our results showed that both DBH and SPI formed protein aggregates that meet the criteria of amyloid fibrils: high content of $\beta$-sheet structure, fibrillar morphology, and high Thioflavin T (Th T) fluorescence intensity. In addition, we found this amyloid fibrillation of both CSPI and DBH was a multistep process, following a sequence of preparatory stage, emergence stage, development stage, and reverse stage. At these 
conditions, we observed that CSPI formed shorter and worm-like fibrils while DBH formed longer and semiflexible fibrils. These results, for the first time, showed that amyloid fibrils can be formed by soy protein enzymatic hydrolysates, and selectively hydrolyzing $\beta$ conglycinin changed the morphology of soy protein fibrils. The long and semiflexible fibrils formed by $\mathrm{D} B \mathrm{H}$ could be a promising thickening agent or structural ingredients.

In Chapter 3, selective proteolysis was applied on soy protein again, but targeted at the glycinin component, thus a degraded glycinin hydrolysate (DGH) was obtained. We further treated this DGH and non-hydrolyzed control SPI with high intensity ultrasound (HIU) (20 $\mathrm{kHz}$ at $400 \mathrm{~W}, 0,5,20$, and $40 \mathrm{~min}$ ), a physical modification approach that has drawn increasing attention in food protein science. Since HIU was reported to have limited effects on isolated glycinin, we aimed to explore the combined effects of selective proteolysis of glycinin and HIU on soy protein. Our results showed that the physical, structural, and aggregation properties of DGH and non-hydrolyzed SPI were both dependent on the HIU treatment time. At an early stage, HIU treatment induced these proteins to form soluble aggregates, while prolonged HIU treatment dissociated these newly formed aggregates again. Selectively hydrolyzing glycinin contributed to the formation of soluble aggregates under the subsequent HIU treatment, which could be attributed to the unfolding of the compact glycinin structure, the generation of small protein fractions, and the increased ratio of $\beta$-conglycinin: glycinin. As a result, under the same time of HIU treatment, DGH displayed higher solubility and better gelling ability than CSPI, proving the synergistic effects of selective proteolysis and HIU modifications on soy proteins.

In Chapter 4, we prepared both $\mathbf{D} \boldsymbol{\beta} \mathbf{H}$ and $\mathbf{D G H}$ based on the methods used in the above chapters and compared these two distinct hydrolysates with native SPI in terms of heatinduced gelation properties. Heat-set gels were formed by SPI, hydrolysates, and dialyzed hydrolysates at different protein concentrations (6\%, 10\%, 14\%). To thoroughly investigate the rheological properties of these gels, both small amplitude oscillatory shear (SAOS) and large amplitude oscillatory shear (LAOS) tests were applied, as such we were able to show, for the first time, the complete "rheological fingerprint" of soy protein heat-set gels. Our results showed that both $D \beta H$ and DGH samples could form heat-set gels at a shorter time and lower temperature, compared to native SPI. However, the existence of non-network peptides made hydrolysate gels generally less elastic and stretchable than SPI gels. Selectively hydrolyzing $\beta$-conglycinin to a limited extent (lower $\mathrm{DH}$ ) allowed $\mathrm{D} \beta \mathrm{H}$ to form a stiffer gel than native SPI at a shorter gelling time, which makes it a promising gelling agent in meat products. On the other hand, hydrolyzing glycinin made DGH form weaker gels than SPI, regardless of the $\mathrm{DH}$ and protein concentrations. We also found the gels formed by hydrolysates and native SPI showed different non-linear rheological behaviors. D $\beta H$ gels displayed intercycle strain softening behavior (Type I), while DGH and SPI displayed a weak 
(Type III) and strong (Type IV) intercycle strain overshoot, respectively. By applying Lissajous plots, we revealed that in the medium strain range, SPI showed stronger intracycle shear stiffening, while $D \beta H$ gels showed stronger intracycle shear thinning. At the large strain regime, SPI and DBH gels showed abrupt yielding behavior while DGH gels yielded more gradually. By applying fractal scaling theory, confocal laser scanning microscopy (CLSM) and scanning electron microscopy (SEM), we found that D $\beta H$ gels are weak-link type gels with higher $D_{f}$, while DGH gels are strong-link type gels with lower $D_{f}$. SPI gels are transition type gels with a $D_{f}$ in between. Consistently, D $\beta H$ and SPI gels showed relatively denser networks which were built by large and compact flocs, while DGH gels showed a more coarse and heterogeneous structure, with smaller and more branched constituent flocs. These different gel microstructures could explain the different rheological behaviors of these gels in both in linear and non-linear regimes.

As a benchmark for plant-based proteins, the research on soy protein shares a same final goal: increasing the usage of soy protein in food products or the human diet, which is still mainly based on animal-based proteins. To achieve this goal, understanding the aggregation and gelation behavior of soy protein when it is mixed with animal-based proteins is essential. In Chapter 5, we explored how substituting whey protein isolate (WPI) with SPI affects the rheological behavior and microstructure of acid (GDL)-induced gels. Before gelation was induced by GDL, commercial SPI and WPI dispersions ( $\mathrm{pH} 7.0,3.0 \mathrm{mS} / \mathrm{cm}$ ) were preheated (95 $\left.{ }^{\circ} \mathrm{C}, 30 \mathrm{~min}\right)$ at different total concentrations $(2 \%, 4 \%, 6 \%$, and $8 \% \mathrm{w} / \mathrm{w}$ ) and SPI: WPI ratios $(0: 4,1: 3,2: 2,3: 1$, and $4: 0)$. During this preheating step, we found that more insoluble aggregates were formed while fewer soluble aggregates were formed when the SPI: WPI ratio was higher. The electrostatic repulsion between these protein aggregates could be similar as they showed similar zeta-potential, while the hydrophobic interactions could be stronger when the proportion of SPI was higher, as the surface hydrophobicity of samples increased with increasing SPI: WPI ratio. Subsequently, GDL-induced gels were prepared with these mixed protein aggregates, and both SAOS and LAOS tests were used to provide a full picture of the rheological properties of these cold-set gels. With increasing SPI: WPI ratios, the acidification rate and gelling rate of samples were decreased, while the resultant gels displayed lower strength (lower $\mathrm{G}^{\prime}$ ), stretchability (lower $\gamma_{c}$ ), and water holding capacity (WHC). By applying Lissajous plots, we were able to reveal, for the first time, that the rheological behaviors of the hybrid gels in the non-linear viscoelastic (NLVE) regime were more similar to SPI gels than to WPI gels. As the strain amplitude increased, gels that contained SPI displayed a more gradual transition from elastic behavior to the plastic behavior, and a relatively more elastic response in large strain amplitude, compared to WPI gels. By applying fractal scaling theory, multiphoton laser scanning microscopy (MLSM) and scanning electron microscopy (SEM), we found that WPI gels formed a dense, homogeneous fine-stranded gel network, in which the constituent flocs were strongly 
interconnected. Gels that contained SPI showed significant coarser gel networks which were classified as intermediate type gels. With increased SPI content, more gaps and pores were observed in the gel network, and the constituent flocs became larger, more elongated, and irregularly branched. These significant microstructural changes could be the reason behind the changes in rheological properties induced by replacing WPI with SPI.

This thesis concluded in Chapter 6, in the context of a general discussion tying together the main findings in each chapter, and comparing them with relevant studies in the literature. The effects of several novel methods, i.e. selective proteolysis, ultrasound, and blending with whey protein, on the properties of soy proteins were discussed in terms of amyloid fibrillation, heat-induced, and acid-induced gelation. The results highlighted that selective proteolysis could modify the aggregation and gelation properties of soy proteins more efficiently compared to traditional proteolysis. The link between the rheological properties of soy protein gels with their microstructures was established, and fractal scaling theory proved to be a useful tool. We also concluded that the non-linear rheological properties could provide additional detailed information on protein hydrogel microstructures. Overall, the modification of soy protein cannot be a "one-size-fit-all" approach. Which method to choose, depends largely on the application one is considering, and on several other factors. Large amplitude oscillatory shear (LAOS) tests should be standard in studying the rheological properties of protein gels. The present thesis provided new knowledge on the aggregation and gelation of soy proteins, and also deeper understanding of the interplay between protein modification, rheological properties and gel microstructures, which benefits the application of soy proteins as well as the studies on other plant-based proteins. 



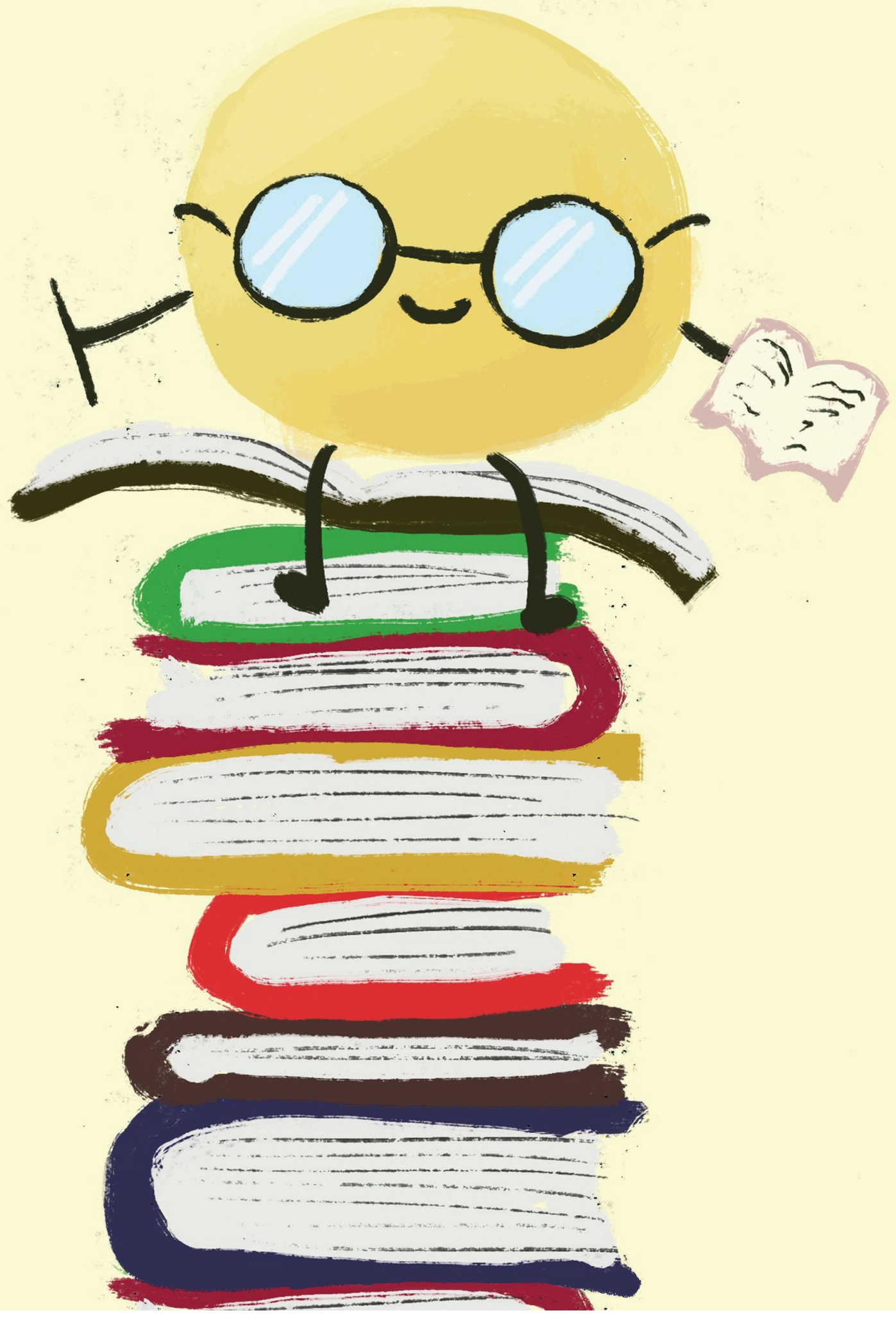




\section{References}

[1] United Nations 2030 Agenda, Transforming Our World: The 2030 Agenda for Sustainable Development, A New Era Glob. Heal. (2018). doi:10.1891/9780826190123.ap02.

[2] J. Guo, X.Q. Yang, X.T. He, N.N. Wu, J.M. Wang, W. Gu, Y.Y. Zhang, Limited aggregation behavior of $\beta$-conglycinin and its terminating effect on glycinin aggregation during heating at pH 7.0, J. Agric. Food Chem. 60 (2012) 3782-3791. doi:10.1021/jf300409y.

[3] R.A. Badley, D. Atkinson, H. Hauser, D. Oldani, J.P. Green, J.M. Stubbs, The structure, physical and chemical properties of the soy bean protein glycinin, BBA Protein Struct. 412 (1975) 214-228. doi:10.1016/0005-2795(75)90036-7.

[4] S. Damodaran, J.E. Kinsella, Effect of Conglycinin on the Thermal Aggregation of Glycinin, J. Agric. Food Chem. 30 (1982) 812-817. doi:10.1021/jf00113a003.

[5] M.R. Nilsson, Techniques to study amyloid fibril formation in vitro, Methods. 34 (2004) 151-160. doi:10.1016/j.ymeth.2004.03.012.

[6] C. Akkermans, A.J. Van Der Goot, P. Venema, H. Gruppen, J.M. Vereijken, E. Van Der Linden, R.M. Boom, Micrometer-sized fibrillar protein aggregates from soy glycinin and soy protein isolate, J. Agric. Food Chem. 55 (2007) 9877-9882. doi:10.1021/jf0718897.

[7] C.H. Tang, C.S. Wang, Formation and characterization of amyloid-like fibrils from soy $\beta$-conglycinin and glycinin, J. Agric. Food Chem. 58 (2010) 11058-11066. doi:10.1021/jf1021658.

[8] Y. Wang, Y. Shen, G. Qi, Y. Li, X.S. Sun, D. Qiu, Y. Li, Formation and physicochemical properties of amyloid fibrils from soy protein, Int. J. Biol. Macromol. 149 (2020) 609-616. doi:10.1016/j.ijbiomac.2020.01.258.

[9] T. Nakamura, S. Utsumi, T. Mori, Network Structure Formation in Thermally Induced Gelation of Glycinin, J. Agric. Food Chem. 32 (1984) 349-352. doi:10.1021/jf00122a042.

[10] J.M.S. Renkema, J.H.M. Knabben, T. Van Vliet, Gel formation by $\beta$-conglycinin and glycinin and their mixtures, Food Hydrocoll. 15 (2001) 407-414. doi:10.1016/S0268-005X(01)00051-0. 
[11] J.M.S. Renkema, C.M.M. Lakemond, H.H.J. De Jongh, H. Gruppen, T. Van Vliet, The effect of $\mathrm{pH}$ on heat denaturation and gel forming properties of soy proteins, $\mathrm{J}$. Biotechnol. 79 (2000) 223-230. doi:10.1016/S0168-1656(00)00239-X.

[12] J.M.S. Renkema, H. Gruppen, T. Van Vliet, Influence of $\mathrm{pH}$ and ionic strength on heat-induced formation and rheological properties of soy protein gels in relation to denaturation and their protein compositions, J. Agric. Food Chem. 50 (2002) 60646071. doi:10.1021/jf020061b.

[13] J.M.S. Renkema, T. Van Vliet, Heat-induced gel formation by soy proteins at neutral pH, J. Agric. Food Chem. 50 (2002) 1569-1573. doi:10.1021/jf010763I.

[14] T. Nagano, H. Mori, K. Nishinari, Effect of Heating and Cooling on the Gelation Kinetics of 7S Globulin from Soybeans, J. Agric. Food Chem. 42 (1994) 1415-1419. doi: 10.1021/jf00043a005.

[15] C.M.M. Lakemond, H.H.J. De Jongh, M. Paques, T. Van Vliet, H. Gruppen, A.G.J. Voragen, Gelation of soy glycinin; influence of $\mathrm{pH}$ and ionic strength on network structure in relation to protein conformation, Food Hydrocoll. 17 (2003) 365-377. doi: 10.1016/S0268-005X(02)00100-5.

[16] J.M.S. Renkema, Relations between rheological properties and network structure of soy protein gels, Food Hydrocoll. 18 (2004) 39-47. doi:10.1016/S0268005X(03)00040-7.

[17] A. Maltais, G.E. Remondetto, M. Subirade, Mechanisms involved in the formation and structure of soya protein cold-set gels: A molecular and supramolecular investigation, Food Hydrocoll. $22 \quad$ (2008) 550-559. doi:10.1016/j.foodhyd.2007.01.026.

[18] A. Maltais, G.E. Remondetto, M. Subirade, Soy protein cold-set hydrogels as controlled delivery devices for nutraceutical compounds, Food Hydrocoll. 23 (2009) 1647-1653. doi:10.1016/j.foodhyd.2008.12.006.

[19] A. Maltais, G.E. Remondetto, M. Subirade, Tabletted soy protein cold-set hydrogels as carriers of nutraceutical substances, Food Hydrocoll. 24 (2010) 518-524. doi:10.1016/j.foodhyd.2009.11.016.

[20] A. Maltais, G.E. Remondetto, R. Gonzalez, M. Subirade, Formation of soy protein isolate cold-set gels: Protein and salt effects, J. Food Sci. 70 (2005) C67-C73. doi:10.1111/j.1365-2621.2005.tb09023.x. 
[21] L.J. Campbell, X. Gu, S.J. Dewar, S.R. Euston, Effects of heat treatment and glucono- $\delta$-lactone-induced acidification on characteristics of soy protein isolate, Food Hydrocoll. 23 (2009) 344-351. doi:10.1016/j.foodhyd.2008.03.004.

[22] S. Schuldt, N. Raak, D. Jaros, H. Rohm, Acid-induced formation of soy protein gels in the presence of $\mathrm{NaCl}$, LWT - Food Sci. Technol. 57 (2014) 634-639. doi:10.1016/j.Iwt.2014.02.013.

[23] Y. Wan, Y. Li, S. Guo, Characteristics of soy protein isolate gel induced by glucono$\delta$-lactone: Effects of the protein concentration during preheating, Food Hydrocoll. 113 (2021). doi:10.1016/j.foodhyd.2020.106525.

[24] T. Kanchan, K. Krishan, Questioning the impact of journal impact factor on research?, Biomed. J. 38 (2015) 462. doi:10.4103/2319-4170.165000.

[25] R.H. Ewoldt, A.E. Hosoi, G.H. McKinley, New measures for characterizing nonlinear viscoelasticity in large amplitude oscillatory shear, J. Rheol. (N. Y. N. Y). 52 (2008) 1427-1458. doi:10.1122/1.2970095.

[26] W.H. Shih, W.Y. Shih, S. Il Kim, J. Liu, I.A. Aksay, Scaling behavior of the elastic properties of colloidal gels, Phys. Rev. A. 42 (1990) 4772-4779. doi:10.1103/PhysRevA.42.4772.

[27] H. Wu, M. Morbidelli, Model relating structure of colloidal gels to their elastic properties, Langmuir. 17 (2001) 1030-1036. doi:10.1021/la001121f.

[28] A.P. Williams, Enzymic hydrolysis of food proteins, Elsevier applied science publishers, 1987. doi:10.1016/0308-8146(87)90169-5.

[29] S.R. Drago, R.J. González, Foaming properties of enzymatically hydrolysed wheat gluten, Innov. Food Sci. Emerg. Technol. 1 (2000) 269-273. doi:10.1016/S14668564(00)00034-5.

[30] D.J.L. Mat, T. Cattenoz, I. Souchon, C. Michon, S. Le Feunteun, Monitoring protein hydrolysis by pepsin using $\mathrm{pH}$-stat: In vitro gastric digestions in static and dynamic pH conditions, Food Chem. 239 (2018) 268-275. doi:10.1016/j.foodchem.2017.06.115.

[31] K. Tsumura, T. Saito, W. Kugimiya, K. Inouye, Selective proteolysis of the glycinin and $\beta$-conglycinin fractions in a soy protein isolate by pepsin and papain with controlled pH and temperature, J. Food Sci. 69 (2004) C363-C367. doi:10.1111/j.1365-2621.2004.tb10698.x. 
[32] F. Chemat, Zill-E-Huma, M.K. Khan, Applications of ultrasound in food technology: Processing, preservation and extraction, Ultrason. Sonochem. 18 (2011) 813-835. doi:10.1016/j.ultsonch.2010.11.023.

[33] K. Nishinari, Y. Fang, S. Guo, G.O. Phillips, Soy proteins: A review on composition, aggregation and emulsification, Food Hydrocoll. 39 (2014) 301-318. doi:10.1016/j.foodhyd.2014.01.013.

[34] B. Li, Z. Bao, W. Xu, Y. Chi, Influence of glycation extent on the physicochemical and gelling properties of soybean $\beta$-conglycinin, Eur. Food Res. Technol. 240 (2015) 399-411. doi:10.1007/s00217-014-2339-0.

[35] V.M. Pizones Ruiz-Henestrosa, M.J. Martinez, C. Carrera Sánchez, J.M. Rodríguez Patino, A.M.R. Pilosof, Mixed soy globulins and $\beta$-lactoglobulin systems behaviour inaqueous solutions and at the air-water interface, Food Hydrocoll. 35 (2014) 106114. doi:10.1016/j.foodhyd.2013.04.021.

[36] D.B. Yuan, W. Min, X.Q. Yang, C.H. Tang, K.L. Huang, J. Guo, J.M. Wang, N.N. Wu, H.G. Zheng, J.R. Qi, An improved isolation method of soy $\beta$-conglycinin subunits and their characterization, JAOCS, J. Am. Oil Chem. Soc. 87 (2010) 997-1004. doi:10.1007/s11746-010-1583-0.

[37] H.G. Zheng, X.Q. Yang, I. Ahmad, W. Min, J.H. Zhu, D.B. Yuan, Soybean $\beta-$ conglycinin constituent subunits: Isolation, solubility and amino acid composition, Food Res. Int. 42 (2009) 998-1003. doi:10.1016/j.foodres.2009.04.018.

[38] K. Tsumura, Improvement of the physicochemical properties of soybean proteins by enzymatic hydrolysis, Food Sci. Technol. Res. 15 (2009) 381-388. doi:10.3136/fstr.15.381.

[39] W. Margatan, K. Ruud, Q. Wang, T. Markowski, B. Ismail, Angiotensin converting enzyme inhibitory activity of soy protein subjected to selective hydrolysis and thermal processing, J. Agric. Food Chem. 61 (2013) 3460-3467. doi: $10.1021 / \mathrm{jf} 4001555$.

[40] K. Tsumura, T. Saito, K. Tsuge, H. Ashida, W. Kugimiya, K. Inouye, Functional properties of soy protein hydrolysates obtained by selective proteolysis, LWT - Food Sci. Technol. 38 (2005) 255-261. doi:10.1016/j.Iwt.2004.06.007.

[41] H. Choi, H.J. Chang, Y. Shin, J.I. Kim, H.S. Park, G. Yoon, S. Na, The molecular mechanism of conformational changes of the triplet prion fibrils for $\mathrm{pH}, \mathrm{RSC}$ Adv. 5 
(2015) 49263-49269. doi:10.1039/c5ra08015k.

[42] W. Lee, H. Jung, M. Son, H. Lee, T.J. Kwak, G. Lee, C.H. Kim, S.W. Lee, D.S. Yoon, Characterization of the regrowth behavior of amyloid-like fragmented fibrils decomposed by ultrasonic treatment, RSC Adv. 4 (2014) 56561-56566. doi: $10.1039 / c 4 r a 08270 b$.

[43] J. Liu, C.H. Tang, Heat-induced fibril assembly of vicilin at pH2.0: Reaction kinetics, influence of ionic strength and protein concentration, and molecular mechanism, Food Res. Int. 51 (2013) 621-632. doi:10.1016/j.foodres.2012.12.049.

[44] M.G. Santangelo, V. Foderà, V. Militello, V. Vetri, Back to the oligomeric state: PHinduced dissolution of concanavalin A amyloid-like fibrils into non-native oligomers, RSC Adv. 6 (2016) 75082-75091. doi:10.1039/c6ra16690c.

[45] J.R. Wagner, D.A. Sorgentini, M.C. Anon, Relation between solubility and surface hydrophobicity as an indicator of modifications during preparation processes of commercial and laboratory-prepared soy protein isolates, J. Agric. Food Chem. 48 (2000) 3159-3165. doi:10.1021/jf990823b.

[46] F. Chemistry, Improved Method for Determining, J. Food Sci. 66 (2001) 642-646.

[47] U.K. Laemmli, Cleavage of structural proteins during the assembly of the head of bacteriophage T4, Nature. 227 (1970) 680-685. doi:10.1038/227680a0.

[48] C.H. Tang, Y.H. Zhang, Q.B. Wen, Q. Huang, Formation of amyloid fibrils from kidney bean 7s globulin (phaseolin) at pH 2.0, J. Agric. Food Chem. 58 (2010) 8061-8068. doi:10.1021/jf101311f.

[49] E.M. Bainy, S.M. Tosh, M. Corredig, L. Woodrow, V. Poysa, Protein subunit composition effects on the thermal denaturation at different stages during the soy protein isolate processing and gelation profiles of soy protein isolates, JAOCS, J. Am. Oil Chem. Soc. 85 (2008) 581-590. doi:10.1007/s11746-008-1238-6.

[50] A.M. Nik, S.M. Tosh, V. Poysa, L. Woodrow, M. Corredig, Protein recovery in soymilk and various soluble fractions as a function of genotype differences, changes during heating, and homogenization, J. Agric. Food Chem. 56 (2008) 10893-10900. doi:10.1021/jf800897s.

[51] A. Malaki Nik, S.M. Tosh, L. Woodrow, V. Poysa, M. Corredig, Effect of soy protein subunit composition and processing conditions on stability and particle size distribution of soymilk, LWT - Food Sci. Technol. 42 (2009) 1245-1252. 
doi:10.1016/j.Iwt.2009.03.001.

[52] aleksander ilic vera popovic, jegor miladinovic, milos vidic, vojislav mihailovic, jela ikanovic, Genotipe X Environment Interaction Between Yield and Quality Components of Soybean (Glycine Max), Agric. For. 60 (2014) 33-46.

[53] V. Poysa, L. Woodrow, K. Yu, Effect of soy protein subunit composition on tofu quality, Food Res. Int. 39 (2006) 309-317. doi:10.1016/j.foodres.2005.08.003.

[54] K. Taski-Ajdukovic, V. Djordjevic, M. Vidic, M. Vujakovic, Composição de subunidades de proteínas de reserva em genótipos de soja com alto teor de proteína, Pesqui. Agropecu. Bras. 45 (2010) 721-729. doi:10.1590/S0100204X2010000700013.

[55] L.N. Arnaudov, R. de Vries, H. Ippel, C.P.M. van Mierlo, Multiple steps during the formation of $\beta$-lactoglobulin fibrils, Biomacromolecules. 4 (2003) 1614-1622. doi: $10.1021 / \mathrm{bm034096b.}$

[56] A. Kroes-Nijboer, P. Venema, J. Bouman, E. Van Der Linden, Influence of protein hydrolysis on the growth kinetics of $\beta$-Ig fibrils, Langmuir. 27 (2011) 5753-5761. doi:10.1021/la104797u.

[57] C. Akkermans, P. Venema, A.J. van der Goot, H. Gruppen, E.J. Bakx, R.M. Boom, E. van der Linden, Peptides are building blocks of heat-induced fibrillar protein aggregates of $\beta$-lactoglobulin formed at pH 2, Biomacromolecules. 9 (2008) 14741479. doi: $10.1021 / \mathrm{bm} 7014224$.

[58] S.G. Bolder, A.J. Vasbinder, L.M.C. Sagis, E. van der Linden, Heat-induced whey protein isolate fibrils: Conversion, hydrolysis, and disulphide bond formation, Int. Dairy J. 17 (2007) 846-853. doi:10.1016/j.idairyj.2006.10.002.

[59] R. Mishra, K. Sörgjerd, S. Nyström, A. Nordigården, Y.C. Yu, P. Hammarström, Lysozyme Amyloidogenesis Is Accelerated by Specific Nicking and Fragmentation but Decelerated by Intact Protein Binding and Conversion, J. Mol. Biol. 366 (2007) 1029-1044. doi:10.1016/j.jmb.2006.11.084.

[60] Q. Zhong, C.R. Daubert, O.D. Velev, Physicochemical variables affecting the rheology and microstructure of rennet casein gels, J. Agric. Food Chem. 55 (2007) 2688-2697. doi:10.1021/jf0625914.

[61] G.D. Rose, L.M. Glerasch, J.A. Smith, Turns in Peptides and Proteins, Adv. Protein Chem. 37 (1985) 1-109. doi:10.1016/S0065-3233(08)60063-7. 
[62] A.H. CLARK, D.H.P. SAUNDERSON, A. SUGGETT, Infrared and Laser-Raman Spectroscopic Studies of Thermally-Induced Globular Protein Gels, Int. J. Pept. Protein Res. 17 (1981) 353-364. doi:10.1111/j.1399-3011.1981.tb02002.x.

[63] F. Meersman, L. Smeller, K. Heremans, Comparative Fourier transform infrared spectroscopy study of cold-, pressure-, and heat-induced unfolding and aggregation of myoglobin, Biophys. J. 82 (2002) 2635-2644. doi:10.1016/S00063495(02)75605-1.

[64] B. Jachimska, M. Wasilewska, Z. Adamczyk, Characterization of globular protein solutions by dynamic light scattering, electrophoretic mobility, and viscosity measurements, Langmuir. 24 (2008) 6867-6872. doi:10.1021/la800548p.

[65] B.M. McKenna, Functional properties of food macromolecules, Springer Science \& Business Media, 1988. doi:10.1016/0260-8774(88)90038-6.

[66] B. Zhang, X. Guo, K. Zhu, W. Peng, H. Zhou, Improvement of emulsifying properties of oat protein isolate-dextran conjugates by glycation, Carbohydr. Polym. 127 (2015) 168-175. doi:10.1016/j.carbpol.2015.03.072.

[67] A.C. Krämer, P.W. Thulstrup, M.N. Lund, M.J. Davies, Key role of cysteine residues and sulfenic acids in thermal-and H2O2-mediated modification of $\beta$-lactoglobulin, $\begin{array}{lllll}\text { Free } \quad \text { Radic. } & \text { Biol. } & \text { (2016) } & \text { 544-555. }\end{array}$ doi:10.1016/j.freeradbiomed.2016.07.010.

[68] L. Wang, M. Zhang, Z. Fang, B. Bhandari, Gelation properties of myofibrillar protein under malondialdehyde-induced oxidative stress, J. Sci. Food Agric. 97 (2017) 5057. doi:10.1002/jsfa.7680.

[69] T. Grune, Oxidative stress, aging and the proteasomal system, Biogerontology. 1 (2000) 31-40. doi:10.1023/A:1010037908060.

[70] E.N.C. Mills, L. Huang, T.R. Noel, A.P. Gunning, V.J. Morris, Formation of thermally induced aggregates of the soya globulin $\beta$-conglycinin, Biochim. Biophys. Acta Protein Struct. Mol. Enzymol. 1547 (2001) 339-350. doi:10.1016/S01674838(01)00199-6.

[71] M.C. Puppo, M.C. Añón, Structural Properties of Heat-Induced Soy Protein Gels As Affected by Ionic Strength and pH, J. Agric. Food Chem. 46 (1998) 3583-3589. doi:10.1021/jf980006w.

[72] L.M.C. Sagis, C. Veerman, E. Van Der Linden, Mesoscopic properties of semiflexible 
amyloid fibrils, Langmuir. 20 (2004) 924-927. doi:10.1021/la035390s.

[73] S.M. Saeed, G. Fine, Thioflavin-T for amyloid detection., Am. J. Clin. Pathol. 47 (1967) 588-593. doi:10.1093/ajcp/47.5.588.

[74] S.A. Hudson, H. Ecroyd, T.W. Kee, J.A. Carver, The thioflavin T fluorescence assay for amyloid fibril detection can be biased by the presence of exogenous compounds, FEBS J. 276 (2009) 5960-5972. doi:10.1111/j.1742-4658.2009.07307.x.

[75] A. Kroes-Nijboer, Y.S. Lubbersen, P. Venema, E. van der Linden, Thioflavin T fluorescence assay for $\beta$-lactoglobulin fibrils hindered by DAPH, J. Struct. Biol. 165 (2009) 140-145. doi:10.1016/j.jsb.2008.11.003.

[76] R. Sabaté, S.J. Saupe, Thioflavin T fluorescence anisotropy: An alternative technique for the study of amyloid aggregation, Biochem. Biophys. Res. Commun. 360 (2007) 135-138. doi:10.1016/j.bbrc.2007.06.063.

[77] M. Zhu, S. Han, F. Zhou, S.A. Carter, A.L. Fink, Annular oligomeric amyloid intermediates observed by in situ atomic force microscopy, J. Biol. Chem. 279 (2004) 24452-24459. doi:10.1074/jbc.M400004200.

[78] R. Sabate, L. Rodriguez-Santiago, M. Sodupe, S.J. Saupe, S. Ventura, Thioflavin-T excimer formation upon interaction with amyloid fibers, Chem. Commun. 49 (2013) 5745-5747. doi:10.1039/c3cc42040j.

[79] K.N. Ryan, E.A. Foegeding, Formation of soluble whey protein aggregates and their stability in beverages, Food Hydrocoll. 43 (2015) 265-274. doi:10.1016/j.foodhyd.2014.05.025.

[80] C. Schmitt, C. Bovay, A.M. Vuilliomenet, M. Rouvet, L. Bovetto, R. Barbar, C. Sanchez, Multiscale characterization of individualized $\beta$-lactoglobulin microgels formed upon heat treatment under narrow $\mathrm{pH}$ range conditions, Langmuir. 25 (2009) 7899-7909. doi:10.1021/la900501n.

[81] C. Schmitt, C. Moitzi, C. Bovay, M. Rouvet, L. Bovetto, L. Donato, M.E. Leser, P. Schurtenberger, A. Stradner, Internal structure and colloidal behaviour of covalent whey protein microgels obtained by heat treatment, Soft Matter. 6 (2010) 48764884. doi:10.1039/c0sm00220h.

[82] J. Romero, S.-M.M. Sun, R.C. McLeester, F.A. Bliss, T.C. Hall, Heritable Variation in a Polypeptide Subunit of the Major Storage Protein of the Bean, Phaseolus vulgaris L. , Plant Physiol. 56 (1975) 776-779. doi:10.1104/pp.56.6.776. 
[83] C. Veerman, H. Ruis, L.M.C. Sagis, E. van der Linden, Effect of electrostatic interactions on the percolation concentration of fibrillar $\beta$-lactoglobulin gels, Biomacromolecules. 3 (2002) 869-873. doi:10.1021/bm025533+.

[84] M.I. Viseu, T.I. Carvalho, S.M.B. Costa, Conformational Transitions in $\beta$ Lactoglobulin Induced by Cationic Amphiphiles: Equilibrium Studies, Biophys. J. 86 (2004) 2392-2402. doi:10.1016/S0006-3495(04)74296-4.

[85] R.W. Alston, M. Lasagna, G.R. Grimsley, J.M. Scholtz, G.D. Reinhart, C.N. Pace, Tryptophan fluorescence reveals the presence of long-range interactions in the denatured state of Ribonuclease Sa, Biophys. J. 94 (2008) 2288-2296. doi:10.1529/biophysj.107.116954.

[86] C.M.M. Lakemond, H.H.J. De Jongh, M. Hessing, H. Gruppen, A.G.J. Voragen, Soy glycinin: Influence of $\mathrm{pH}$ and ionic strength on solubility and molecular structure at ambient temperatures, J. Agric. Food Chem. 48 (2000) 1985-1990. doi:10.1021/jf9908695.

[87] K. Deng, Y. Huang, Y. Hua, Isolation of glycinin (11S) from lipid-reduced soybean flour: Effect of processing conditions on yields and purity, Molecules. 17 (2012) 2968-2979. doi:10.3390/molecules17032968.

[88] N. Traverso, S. Menini, E.P. Maineri, S. Patriarca, P. Odetti, D. Cottalasso, U.M. Marinari, M.A. Pronzato, Malondialdehyde, a lipoperoxidation-derived aldehyde, can bring about secondary oxidative damage to proteins, Journals Gerontol. - Ser. A Biol. Sci. Med. Sci. 59 (2004) 890-895. doi:10.1093/gerona/59.9.b890.

[89] W. Wu, C. Zhang, X. Kong, Y. Hua, Oxidative modification of soy protein by peroxyl radicals, Food Chem. 116 (2009) 295-301. doi:10.1016/j.foodchem.2009.02.049.

[90] J.A. Téllez-Morales, B. Hernández-Santo, J. Rodríguez-Miranda, Effect of ultrasound on the techno-functional properties of food components/ingredients: A review, Ultrason. Sonochem. 61 (2020). doi:10.1016/j.ultsonch.2019.104787.

[91] M. Zhou, J. Liu, Y. Zhou, X. Huang, F. Liu, S. Pan, H. Hu, Effect of high intensity ultrasound on physicochemical and functional properties of soybean glycinin at different ionic strengths, Innov. Food Sci. Emerg. Technol. 34 (2016) 205-213. doi:10.1016/j.ifset.2016.02.007.

[92] A.R. Jambrak, V. Lelas, T.J. Mason, G. Krešić, M. Badanjak, Physical properties of ultrasound treated soy proteins, J. Food Eng. 93 (2009) 386-393. doi:10.1016/j.jfoodeng.2009.02.001. 
[93] C. Arzeni, K. Martínez, P. Zema, A. Arias, O.E. Pérez, A.M.R. Pilosof, Comparative study of high intensity ultrasound effects on food proteins functionality, J. Food Eng. 108 (2012) 463-472. doi:10.1016/j.jfoodeng.2011.08.018.

[94] H. Hu, J. Wu, E.C.Y. Li-Chan, L. Zhu, F. Zhang, X. Xu, G. Fan, L. Wang, X. Huang, S. Pan, Effects of ultrasound on structural and physical properties of soy protein isolate (SPI) dispersions, Food Hydrocoll. 30 (2013) 647-655. doi:10.1016/j.foodhyd.2012.08.001.

[95] T. Zheng, X. Li, A. Taha, Y. Wei, T. Hu, P.B. Fatamorgana, Z. Zhang, F. Liu, X. Xu, S. Pan, H. Hu, Effect of high intensity ultrasound on the structure and physicochemical properties of soy protein isolates produced by different denaturation methods, Food Hydrocoll. $97 \quad$ (2019) 105216. doi:10.1016/j.foodhyd.2019.105216.

[96] H. Hu, I.W.Y. Cheung, S. Pan, E.C.Y. Li-Chan, Effect of high intensity ultrasound on physicochemical and functional properties of aggregated soybean $\beta$-conglycinin and glycinin, Food Hydrocoll. 45 (2015) 102-110. doi:10.1016/j.foodhyd.2014.11.004.

[97] L. Chen, J. Chen, J. Ren, M. Zhao, Effects of ultrasound pretreatment on the enzymatic hydrolysis of soy protein isolates and on the emulsifying properties of hydrolysates, J. Agric. Food Chem. 59 (2011) 2600-2609. doi:10.1021/jf103771x.

[98] W. Li, Y. Wang, H. Zhao, Z. He, M. Zeng, F. Qin, J. Chen, Improvement of emulsifying properties of soy protein through selective hydrolysis: Interfacial shear rheology of adsorption layer, Food Hydrocoll. 60 (2016) 453-460. doi:10.1016/j.foodhyd.2016.04.019.

[99] W. Li, Y. Wang, J. Li, Y. Jiao, J. Chen, Synergistic and competitive effects of monoglycerides on the encapsulation and interfacial shear rheological behavior of soy proteins, Food Hydrocoll. 89 (2019) 631-636. doi:10.1016/j.foodhyd.2018.11.023.

[100] W. Xia, H. Zhang, J. Chen, H. Hu, F. Rasulov, D. Bi, X. Huang, S. Pan, Formation of amyloid fibrils from soy protein hydrolysate: Effects of selective proteolysis on $\beta$ conglycinin, Food Res. Int. $100 \quad$ (2017) 268-276. doi:10.1016/j.foodres.2017.08.059.

[101] L. Huang, X. Ding, Y. Li, H. Ma, The aggregation, structures and emulsifying properties of soybean protein isolate induced by ultrasound and acid, Food Chem. 279 (2019) 114-119. doi:10.1016/j.foodchem.2018.11.147. 
[102] A. Kato, S. Nakai, Hydrophobicity determined by a fluorescence probe method and its correlation with surface properties of proteins., Biochim. Biophys. Acta. 624 (1980) 13-20. doi:10.1016/0005-2795(80)90220-2.

[103] L. Jiang, J. Wang, Y. Li, Z. Wang, J. Liang, R. Wang, Y. Chen, W. Ma, B. Qi, M. Zhang, Effects of ultrasound on the structure and physical properties of black bean protein isolates, Food Res. Int. 62 (2014) 595-601. doi:10.1016/j.foodres.2014.04.022.

[104] E.C.Y. Li-Chan, The applications of Raman spectroscopy in food science, Trends Food Sci. Technol. 7 (1996) 361-370. doi:10.1016/S0924-2244(96)10037-6.

[105] M.C. Puppo, M.C. Añón, Soybean protein dispersions at acid pH. Thermal and rheological properties, J. Food Sci. 64 (1999) 50-56. doi:10.1111/j.13652621.1999.tb09859.x.

[106] C.H. Tang, X.Y. Wang, X.Q. Yang, L. Li, Formation of soluble aggregates from insoluble commercial soy protein isolate by means of ultrasonic treatment and their gelling properties, J. Food Eng. $92 \quad$ (2009) 432-437. doi:10.1016/j.jfoodeng.2008.12.017.

[107] J.M. Wang, N. Xia, X.Q. Yang, S.W. Yin, J.R. Qi, X.T. He, D.B. Yuan, L.J. Wang, Adsorption and dilatational rheology of heat-treated soy protein at the oil-water interface: Relationship to structural properties, J. Agric. Food Chem. 60 (2012) 3302-3310. doi:10.1021/jf205128v.

[108] A. Shanmugam, J. Chandrapala, M. Ashokkumar, The effect of ultrasound on the physical and functional properties of skim milk, Innov. Food Sci. Emerg. Technol. 16 (2012) 251-258. doi:10.1016/j.ifset.2012.06.005.

[109] T. MORI, T. NAKAMURA, S. UTSUMI, Gelation Mechanism of Soybean 11S Globulin: Formation of Soluble Aggregates as Transient Intermediates, J. Food Sci. 47 (1982) 26-30. doi:10.1111/j.1365-2621.1982.tb11019.x.

[110] S. Petruccelli, M.C. Añón, Thermal Aggregation of Soy Protein Isolates, J. Agric. Food Chem. 43 (1995) 3035-3041. doi:10.1021/jf00060a009.

[111] W. Xiong, J. Li, B. Li, L. Wang, Physicochemical properties and interfacial dilatational rheological behavior at air-water interface of high intensity ultrasound modified ovalbumin: Effect of ionic strength, Food Hydrocoll. 97 (2019) 105210. doi:10.1016/j.foodhyd.2019.105210. 
[112] W. Xiong, Y. Wang, C. Zhang, J. Wan, B.R. Shah, Y. Pei, B. Zhou, J. Li, B. Li, High intensity ultrasound modified ovalbumin: Structure, interface and gelation

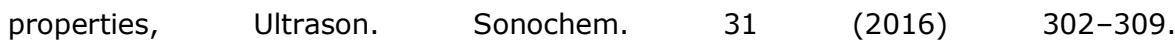
doi:10.1016/j.ultsonch.2016.01.014.

[113] Y. Sun, J. Chen, S. Zhang, H. Li, J. Lu, L. Liu, H. Uluko, Y. Su, W. Cui, W. Ge, L. Jiaping, Effect of power ultrasound pre-treatment on the physical and functional properties of reconstituted milk protein concentrate, J. Food Eng. 124 (2014) 1118. doi:10.1016/j.jfoodeng.2013.09.013.

[114] J. Chandrapala, B. Zisu, M. Palmer, S. Kentish, M. Ashokkumar, Effects of ultrasound on the thermal and structural characteristics of proteins in reconstituted whey protein concentrate, Ultrason. Sonochem. 18 (2011) 951-957. doi:10.1016/j.ultsonch.2010.12.016.

[115] M.A. Malik, C.S. Saini, Heat treatment of sunflower protein isolates near isoelectric point: Effect on rheological and structural properties, Food Chem. 276 (2019) 554561. doi:10.1016/j.foodchem.2018.10.060.

[116] Z. Zhu, W. Zhu, J. Yi, N. Liu, Y. Cao, J. Lu, E.A. Decker, D.J. McClements, Effects of sonication on the physicochemical and functional properties of walnut protein isolate, Food Res. Int. 106 (2018) 853-861. doi:10.1016/j.foodres.2018.01.060.

[117] H. Lee, G. Yildiz, L.C. dos Santos, S. Jiang, J.E. Andrade, N.J. Engeseth, H. Feng, Soy protein nano-aggregates with improved functional properties prepared by sequential pH treatment and ultrasonication, Food Hydrocoll. 55 (2016) 200-209. doi:10.1016/j.foodhyd.2015.11.022.

[118] L. Jiang, Z. Wang, Y. Li, X. Meng, X. Sui, B. Qi, L. Zhou, Relationship between surface hydrophobicity and structure of soy protein isolate subjected to different ionic strength, Int. J. Food Prop. 18 (2015) 1059-1074. doi:10.1080/10942912.2013.865057.

[119] I. Gülseren, D. Güzey, B.D. Bruce, J. Weiss, Structural and functional changes in ultrasonicated bovine serum albumin solutions, Ultrason. Sonochem. 14 (2007) 173-183. doi:10.1016/j.ultsonch.2005.07.006.

[120] J. yu Wang, Y. ling Yang, X. zhi Tang, W. xi Ni, L. Zhou, Effects of pulsed ultrasound on rheological and structural properties of chicken myofibrillar protein, Ultrason. Sonochem. 38 (2017) 225-233. doi:10.1016/j.ultsonch.2017.03.018. 
[121] E.C.Y. Li-Chan, A.A. Ismail, J. Sedman, F.R. van de Voort, Vibrational Spectroscopy of Food and Food Products, in: Handb. Vib. Spectrosc., John Wiley \& Sons, Ltd, Hoboken, New Jersey, 2006. doi:10.1002/0470027320.s6501.

[122] N. Linlaud, E. Ferrer, M.C. Puppo, C. Ferrero, Hydrocolloid interaction with water, protein, and starch in wheat dough, J. Agric. Food Chem. 59 (2011) 713-719. doi:10.1021/jf1026197.

[123] B. Byanju, M.M. Rahman, M.P. Hojilla-Evangelista, B.P. Lamsal, Effect of high-power sonication pretreatment on extraction and some physicochemical properties of proteins from chickpea, kidney bean, and soybean, Int. J. Biol. Macromol. 145 (2020) 712-721. doi:10.1016/j.ijbiomac.2019.12.118.

[124] T. Nagano, H. Mori, K. Nishinari, Rheological properties and conformational states of $\beta$-conglycinin gels at acidic pH, Biopolymers. 34 (1994) 293-298. doi:10.1002/bip.360340215.

[125] A.P. Batista, C.A.M. Portugal, I. Sousa, J.G. Crespo, A. Raymundo, Accessing gelling ability of vegetable proteins using rheological and fluorescence techniques, Int. J. Biol. Macromol. 36 (2005) 135-143. doi:10.1016/j.ijbiomac.2005.04.003.

[126] Y. Cao, R. Mezzenga, Design principles of food gels, Nat. Food. 1 (2020) 106-118. doi:10.1038/s43016-019-0009-x.

[127] C. Wu, W. Ma, Y. Hua, The relationship between breaking force and hydrophobic interactions or disulfide bonds involved in heat-induced soy protein gels as affected by heating time and temperature, Int. J. Food Sci. Technol. 54 (2019) 231-239. doi:10.1111/ijfs.13931.

[128] C. Wu, Y. Hua, Y. Chen, X. Kong, C. Zhang, Effect of temperature, ionic strength and $11 \mathrm{~S}$ ratio on the rheological properties of heat-induced soy protein gels in relation to network proteins content and aggregates size, Food Hydrocoll. 66 (2017) 389-395. doi:10.1016/j.foodhyd.2016.12.007.

[129] N.C. Nielsen, C.D. Dickinson, T.-J. Cho, V.H. Thanh, B.J. Scallon, R.L. Fischer, T.L. Sims, G.N. Drews, R.B. Goldberg, Characterization of the Glycinin Gene Family in Soybean, Plant Cell. 1 (1989) 313. doi:10.2307/3869011.

[130] Y.C. Tseng, Y.L. Xiong, F. Yang, Influence of inulin/oligofructose on the acid-induced cold aggregation and gelation of preheated soy proteins, J. Sci. Food Agric. 89 (2009) 2650-2658. doi:10.1002/jsfa.3770. 
[131] S. Petruccelli, M.C. Añón, Relationship between the Method of Obtention and the Structural and Functional Properties of Soy Protein Isolates. 2. Surface Properties, J. Agric. Food Chem. 42 (1994) 2170-2176. doi:10.1021/jf00046a018.

[132] C. Wu, Y. Hua, Y. Chen, X. Kong, C. Zhang, Effect of 7S/11S ratio on the network structure of heat-induced soy protein gels: a study of probe release, RSC Adv. 6 (2016) 101981-101987. doi:10.1039/c6ra22388e.

[133] T.V. Nieto-Nieto, Y.X. Wang, L. Ozimek, L. Chen, Effects of partial hydrolysis on structure and gelling properties of oat globular proteins, Food Res. Int. 55 (2014) 418-425. doi:10.1016/j.foodres.2013.11.038.

[134] M. Klost, G. Giménez-Ribes, S. Drusch, Enzymatic hydrolysis of pea protein: Interactions and protein fractions involved in fermentation induced gels and their influence on rheological properties, Food Hydrocoll. 105 (2020). doi:10.1016/j.foodhyd.2020.105793.

[135] C. Li, X. Wu, D. Mu, Y. Zhao, S. Luo, X. Zhong, S. Jiang, X. Li, Z. Zheng, Effect of Partial Hydrolysis with Papain on the Characteristics of TransglutaminaseCrosslinked Tofu Gel, J. Food Sci. 83 (2018) 3092-3098. doi:10.1111/17503841.14403.

[136] J.A. Lopes-da-Silva, S.R. Monteiro, Gelling and emulsifying properties of soy protein hydrolysates in the presence of a neutral polysaccharide, Food Chem. 294 (2019) 216-223. doi:10.1016/j.foodchem.2019.05.039.

[137] J. Fan, M. Saito, Z. Yanyan, T. Szesze, L. Wang, E. Tatsumi, L. Li, Gel-forming ability and radical-scavenging activity of soy protein hydrolysate treated with transglutaminase, J. Food Sci. 70 (2005). doi:10.1111/j.13652621.2005.tb09027.x.

[138] E.A. Foegeding, J.P. Davis, Food protein functionality: A comprehensive approach, Food Hydrocoll. 25 (2011) 1853-1864. doi:10.1016/j.foodhyd.2011.05.008.

[139] K. Hyun, M. Wilhelm, C.O. Klein, K.S. Cho, J.G. Nam, K.H. Ahn, S.J. Lee, R.H. Ewoldt, G.H. McKinley, A review of nonlinear oscillatory shear tests: Analysis and application of large amplitude oscillatory shear (LAOS), Prog. Polym. Sci. 36 (2011) 1697-1753. doi:10.1016/j.progpolymsci.2011.02.002.

[140] H.S. Melito, C.R. Daubert, Rheological innovations for characterizing food material properties., Annu. Rev. Food Sci. Technol. 2 (2011) 153-179. doi:10.1146/annurev- 
food-022510-133626.

[141] H.S. Melito, C.R. Daubert, E.A. Foegeding, Relating large amplitude oscillatory shear and food behavior: Correlation of nonlinear viscoelastic, rheological, sensory and oral processing behavior of whey protein isolate/k-carrageenan gels, J. Food Process Eng. 36 (2013) 521-534. doi:10.1111/jfpe.12015.

[142] H.S. Melito, C.R. Daubert, E.A. Foegeding, Relationships between nonlinear viscoelastic behavior and rheological, sensory and oral processing behavior of commercial cheese, J. Texture Stud. 44 (2013) 253-288. doi:10.1111/jtxs.12021.

[143] H.S. Melito, C.R. Daubert, E.A. Foegeding, Validation of a large amplitude oscillatory shear protocol, J. Food Eng. 113 (2012) 124-135. doi:10.1016/j.jfoodeng.2012.05.008.

[144] C. hao Bi, D. Li, L. jun Wang, B. Adhikari, Effect of LBG on the gel properties of acidinduced SPI gels, LWT - Food Sci. Technol. 75 (2017) 1-8. doi:10.1016/j.Iwt.2016.08.028.

[145] C. hao Bi, Y. dan Zhu, L. tong Li, Y. lai Zhang, Z. Hua, J. yi Zhu, Y. Liu, Y. de Liu, Z. gang Huang, Rheological properties and microstructure of soy protein isolate $/ \mathrm{k}$ carrageenan gels under high-speed shear treatment, J. Food Eng. 236 (2018) 4450. doi:10.1016/j.jfoodeng.2018.05.006.

[146] C. hao Bi, M. Zhang, D. yu Sun, Z. Hua, Y. dan Zhu, Y. de Liu, Z. gang Huang, F. Gao, A novel critical point for isotropic gel in rheological-fractal model, J. Food Eng. 244 (2019) 40-46. doi:10.1016/j.jfoodeng.2018.09.023.

[147] S. Precha-Atsawanan, D. Uttapap, L.M.C. Sagis, Linear and nonlinear rheological behavior of native and debranched waxy rice starch gels, Food Hydrocoll. 85 (2018) 1-9. doi:10.1016/j.foodhyd.2018.06.050.

[148] R. Kornet, J. Veenemans, P. Venema, A.J. van der Goot, M. Meinders, L. Sagis, E. van der Linden, Less is more: Limited fractionation yields stronger gels for pea proteins, Food Hydrocoll. 112 (2021) 106285. doi:10.1016/j.foodhyd.2020.106285.

[149] M. Klost, C. Brzeski, S. Drusch, Effect of protein aggregation on rheological properties of pea protein gels, Food Hydrocoll. 108 (2020) 106036. doi:10.1016/j.foodhyd.2020.106036.

[150] F.K.G. Schreuders, L.M.C. Sagis, I. Bodnár, P. Erni, R.M. Boom, A.J. van der Goot, Small and large oscillatory shear properties of concentrated proteins, Food 
Hydrocoll. 110 (2021). doi:10.1016/j.foodhyd.2020.106172.

[151] J. Adler-Nissen, Methods in food protein hydrolysis. In: Enzymic Hydrolysis of Food Protein, Elsevier Applied Science Publishers, New York, NY, 1986.

[152] S.Y. Kim (Lee), P.S.W. Park, K.C. Rhee, Functional Properties of Proteolytic Enzyme Modified Soy Protein Isolate, J. Agric. Food Chem. 38 (1990) 651-656. doi:10.1021/jf00093a014.

[153] J.Y. Lee, H.D. Lee, C.H. Lee, Characterization of hydrolysates produced by mild-acid treatment and enzymatic hydrolysis of defatted soybean flour, Food Res. Int. 34 (2001) 217-222. doi:10.1016/S0963-9969(00)00155-1.

[154] S. Damodaran, Refolding of thermally unfolded soy proteins during the cooling regime of the gelation process: Effect on gelation, J. Agric. Food Chem. 36 (1988) 262-269. doi:10.1021/jf00080a007.

[155] T. Nakamura, S. Utsumi, T. Mori, Interactions during heat-induced gelation in a mixed system of soybean 7s and 11s globulins, Agric. Biol. Chem. 50 (1986) 24292435. doi:10.1080/00021369.1986.10867783.

[156] R. Mujoo, D.T. Trinh, P.K.W. Ng, Characterization of storage proteins in different soybean varieties and their relationship to tofu yield and texture, Food Chem. 82 (2003) 265-273. doi:10.1016/S0308-8146(02)00547-2.

[157] M.Z. Pavlicevic, M.D. Tomic, J.A. Djonlagic, S.P. Stanojevic, B. V. Vucelic Radovic, Evaluation of Variation in Protein Composition on Solubility, Emulsifying and Gelling Properties of Soybean Genotypes Synthesizing $\beta^{\prime}$ Subunit, JAOCS, J. Am. Oil Chem. Soc. 95 (2018) 123-134. doi:10.1002/aocs.12002.

[158] E.J.G. CHACON, L.D. SATTERLEE, M.A. HANNA, Heat Induced Gels From Partially Hydrolyzed Soy Protein Isolate, J. Food Biochem. 14 (1990) 15-29. doi:10.1111/j.1745-4514.1990.tb00818.x.

[159] C. Wu, Y. Hua, Y. Chen, X. Kong, C. Zhang, Release behavior of non-network proteins and its relationship to the structure of heat-induced soy protein gels, J. Agric. Food Chem. 63 (2015) 4211-4219. doi:10.1021/acs.jafc.5b00132.

[160] S. Ikeda, E.A. Foegeding, T. Hagiwara, Rheological study on the fractal nature of the protein gel structure, Langmuir. 15 (1999) 8584-8589. doi:10.1021/la9817415.

[161] T. Hagiwara, H. Kumagai, T. Matsunaga, Fractal Analysis of the Elasticity of BSA and 
$\beta$-Lactoglobulin Gels, J. Agric. Food Chem. 45 (1997) 3807-3812. doi:10.1021/jf970348m.

[162] H. Zhao, B. Yu, Y. Hemar, J. Chen, B. Cui, Improvement of calcium sulfate-induced gelation of soy protein via incorporation of soy oil before and after thermal denaturation, Lwt. 117 (2020) 108690. doi:10.1016/j.Iwt.2019.108690.

[163] K. Shimada, S. Matsushita, Gel formation of soybean $7 \mathrm{~s}$ and $11 \mathrm{~s}$ proteins, Agric. Biol. Chem. 44 (1980) 637-641. doi:10.1080/00021369.1980.10863995.

[164] Y. Zhu, S. Fu, C. Wu, B. Qi, F. Teng, Z. Wang, Y. Li, L. Jiang, The investigation of protein flexibility of various soybean cultivars in relation to physicochemical and conformational properties, Food Hydrocoll. $103 \quad$ (2020) 105709. doi:10.1016/j.foodhyd.2020.105709.

[165] T. Gisler, R.C. Ball, D.A. Weitz, Strain hardening of fractal colloidal gels, Phys. Rev. Lett. 82 (1999) 1064-1067. doi:10.1103/PhysRevLett.82.1064.

[166] J.D. Park, K.H. Ahn, S.J. Lee, Structural change and dynamics of colloidal gels under oscillatory shear flow, Soft Matter. 11 (2015) 9262-9272. doi:10.1039/c5sm01651g.

[167] R.H. Ewoldt, P. Winter, J. Maxey, G.H. McKinley, Large amplitude oscillatory shear of pseudoplastic and elastoviscoplastic materials, Rheol. Acta. 49 (2010) 191-212. doi:10.1007/s00397-009-0403-7.

[168] R.H. Ewoldt, G.H. McKinley, On secondary loops in LAOS via self-intersection of Lissajous-Bowditch curves, Rheol. Acta. 49 (2010) 213-219. doi:10.1007/s00397009-0408-2.

[169] C.F. Diedericks, L. de Koning, V.A. Jideani, P. Venema, E. van der Linden, Extraction, gelation and microstructure of Bambara groundnut vicilins, Food Hydrocoll. 97 (2019) 105226. doi:10.1016/j.foodhyd.2019.105226.

[170] E. Van der Linden, E.A. Foegeding, Gelation. Principles, Models and Applications to Proteins, Elsevier Inc., 2009. doi:10.1016/B978-0-12-374195-0.00002-1.

[171] V. Urbonaite, S. van der Kaaij, H.H.J. de Jongh, E. Scholten, K. Ako, E. van der Linden, L. Pouvreau, Relation between gel stiffness and water holding for coarse and fine-stranded protein gels, Food Hydrocoll. 56 (2016) 334-343. doi:10.1016/j.foodhyd.2015.12.011. 
[172] A. -M Hermansson, Structure of soya glycinin and conglycinin gels, J. Sci. Food Agric. 36 (1985) 822-832. doi:10.1002/jsfa.2740360911.

[173] L. Day, Proteins from land plants - Potential resources for human nutrition and food security, Trends Food Sci. Technol. 32 (2013) 25-42. doi:10.1016/j.tifs.2013.05.005.

[174] C. Wu, T. Wang, C. Ren, W. Ma, D. Wu, X. Xu, L.S. Wang, M. Du, Advancement of food-derived mixed protein systems: Interactions, aggregations, and functional properties, Compr. Rev. Food Sci. Food Saf. 20 (2021) 627-651. doi:10.1111/15414337.12682 .

[175] A.M. Hermansson, Soy protein gelation, J. Am. Oil Chem. Soc. 63 (1986) 658-666. doi: $10.1007 /$ BF02638232.

[176] W. Xia, W.K. Siu, L.M.C. Sagis, Linear and non-linear rheology of heat-set soy protein gels: Effects of selective proteolysis of $\beta$-conglycinin and glycinin, Food Hydrocoll. 120 (2021) 106962. doi:10.1016/j.foodhyd.2021.106962.

[177] T. Nicolai, C. Chassenieux, Heat-induced gelation of plant globulins, Curr. Opin. Food Sci. 27 (2019) 18-22. doi:10.1016/j.cofs.2019.04.005.

[178] A.C. Alting, R.J. Hamer, C.G. De Kruif, R.W. Visschers, Formation of disulfide bonds in acid-induced gels of preheated whey protein isolate, J. Agric. Food Chem. 48 (2000) 5001-5007. doi:10.1021/jf000474h.

[179] C.M. Bryant, D. Julian McClements, Molecular basis of protein functionality with special consideration of cold-set gels derived from hat-denatured whey, Trends Food Sci. Technol. 9 (1998) 143-151. doi:10.1016/S0924-2244(98)00031-4.

[180] A. Abaee, M. Mohammadian, S.M. Jafari, Whey and soy protein-based hydrogels and nano-hydrogels as bioactive delivery systems, Trends Food Sci. Technol. 70 (2017) 69-81. doi:10.1016/j.tifs.2017.10.011.

[181] A. Kharlamova, C. Chassenieux, T. Nicolai, Acid-induced gelation of whey protein aggregates: Kinetics, gel structure and rheological properties, Food Hydrocoll. 81 (2018) 263-272. doi:10.1016/j.foodhyd.2018.02.043.

[182] M. Britten, H.J. Giroux, Acid-induced gelation of whey protein polymers: Effects of $\mathrm{pH}$ and calcium concentration during polymerization, Food Hydrocoll. 15 (2001) 609-617. doi:10.1016/S0268-005X(01)00049-2. 
[183] J.T. de Faria, V.P.R. Minim, L.A. Minim, Evaluating the effect of protein composition on gelation and viscoelastic characteristics of acid-induced whey protein gels, Food Hydrocoll. 32 (2013) 64-71. doi:10.1016/j.foodhyd.2012.12.006.

[184] C. Schmitt, J.V. Silva, L. Amagliani, C. Chassenieux, T. Nicolai, Heat-induced and acid-induced gelation of dairy/plant protein dispersions and emulsions, Curr. Opin. Food Sci. 27 (2019) 43-48. doi:10.1016/j.cofs.2019.05.002.

[185] M.L. Chihi, N. Sok, R. Saurel, Acid gelation of mixed thermal aggregates of pea globulins and $\beta$-lactoglobulin, Food Hydrocoll. 85 (2018) 120-128. doi:10.1016/j.foodhyd.2018.07.006.

[186] J.L. Mession, S. Roustel, R. Saurel, Interactions in casein micelle - Pea protein system (Part II): Mixture acid gelation with glucono-ס-lactone, Food Hydrocoll. 73 (2017) 344-357. doi:10.1016/j.foodhyd.2017.06.029.

[187] A.H. Martin, M.L. De Los Reyes Jiménez, L. Pouvreau, Modulating the aggregation behaviour to restore the mechanical response of acid induced mixed gels of sodium caseinate and soy proteins, Food Hydrocoll. 58 (2016) 215-223. doi:10.1016/j.foodhyd.2016.02.029.

[188] R.R. Roesch, M. Corredig, Study of the effect of soy proteins on the acid-induced gelation of casein micelles, J. Agric. Food Chem. 54 (2006) 8236-8243. doi:10.1021/jf060875i.

[189] H.S. Joyner, Nonlinear (Large-Amplitude Oscillatory Shear) Rheological Properties and Their Impact on Food Processing and Quality, Annu. Rev. Food Sci. Technol. 12 (2021) 591-609. doi:10.1146/annurev-food-061220-100714.

[190] Z. Yang, Y. Hemar, L. Hilliou, E.P. Gilbert, D.J. McGillivray, M.A.K. Williams, S. Chaieb, Nonlinear Behavior of Gelatin Networks Reveals a Hierarchical Structure, Biomacromolecules. 17 (2016) 590-600. doi:10.1021/acs.biomac.5b01538.

[191] T.B. Goudoulas, N. Germann, Phase transition kinetics and rheology of gelatin$\begin{array}{lllll}\text { alginate mixtures, Food Hydrocoll. } 66 & \text { (2017) 49-60. }\end{array}$ doi:10.1016/j.foodhyd.2016.12.018.

[192] J. John, D. Ray, V.K. Aswal, A.P. Deshpande, S. Varughese, Dissipation and strainstiffening behavior of pectin-Ca gels under LAOS, Soft Matter. 15 (2019) 68526866. doi:10.1039/c9sm00709a.

[193] T.H. McCann, L. Guyon, P. Fischer, L. Day, Rheological properties and microstructure 
of soy-whey protein, Food Hydrocoll. $82 \quad$ (2018) 434-441. doi:10.1016/j.foodhyd.2018.04.023.

[194] R. Roesch, M. Juneja, C. Monagle, M. Corredig, Aggregation of soy/milk mixes during acidification, Food Res. Int. 37 (2004) 209-215. doi:10.1016/j.foodres.2003.11.003.

[195] S.G. Anema, Effect of whey protein addition and pH on the acid gelation of heated skim milk, Int. Dairy J. 79 (2018) 5-14. doi:10.1016/j.idairyj.2017.11.008.

[196] A.S. Eissa, S.A. Khan, Acid-induced gelation of enzymatically modified, preheated whey proteins, J. Agric. Food Chem. 53 (2005) 5010-5017. doi:10.1021/jf047957w.

[197] P.N. KOCHER, E.A. FOEGEDING, Microcentrifuge-Based Method for Measuring WaterHolding of Protein Gels, J. Food Sci. 58 (1993) 1040-1046. doi:10.1111/j.13652621.1993.tb06107.x.

[198] L. Chu, L. Yang, J. Li, L. Lin, G. Zheng, Effect of Smilax china L. starch on the gel properties and interactions of calcium sulfate-induced soy protein isolate gel, Int. J. Biol. Macromol. 135 (2019) 127-132. doi:10.1016/j.ijbiomac.2019.05.130.

[199] X. Gu, L.J. Campbell, S.R. Euston, Influence of sugars on the characteristics of glucono- $\delta$-lactone-induced soy protein isolate gels, Food Hydrocoll. 23 (2009) 314326. doi:10.1016/j.foodhyd.2008.01.005.

[200] B.T. Nguyen, C. Chassenieux, T. Nicolai, C. Schmitt, Effect of the pH and $\mathrm{NaCl}$ on the microstructure and rheology of mixtures of whey protein isolate and casein micelles upon heating, Food Hydrocoll. 70 (2017) 114-122. doi:10.1016/j.foodhyd.2017.03.013.

[201] X. Zhou, G. Sala, L.M.C. Sagis, Bulk and interfacial properties of milk fat emulsions stabilized by whey protein isolate and whey protein aggregates, Food Hydrocoll. 109 (2020) 106100. doi:10.1016/j.foodhyd.2020.106100.

[202] G. Wang, M. Guo, Manufacturing technologies of whey protein products, in: Whey Protein Prod. Chem. Funct. Appl., John Wiley \& Sons, Ltd, 2019: pp. 13-37. doi:10.1002/9781119256052.ch2.

[203] K.N. Ryan, B. Vardhanabhuti, D.P. Jaramillo, J.H. van Zanten, J.N. Coupland, E.A. Foegeding, Stability and mechanism of whey protein soluble aggregates thermally treated with salts, Food Hydrocoll. $27 \quad$ (2012) 411-420. doi:10.1016/j.foodhyd.2011.11.006. 
[204] M.J. Spotti, P.A. Loyeau, A. Marangón, H. Noir, A.C. Rubiolo, C.R. Carrara, Influence of Maillard reaction extent on acid induced gels of whey proteins and dextrans, Food Hydrocoll. 91 (2019) 224-231. doi:10.1016/j.foodhyd.2019.01.020.

[205] T. Nicolai, M. Britten, C. Schmitt, $\beta$-Lactoglobulin and WPI aggregates: Formation, structure and applications, Food Hydrocoll. 25 (2011) 1945-1962. doi:10.1016/j.foodhyd.2011.02.006.

[206] A.C. Alting, E.T. Van Der Meulena, J. Hugenholtz, R.W. Visschers, Control of texture of cold-set gels through programmed bacterial acidification, Int. Dairy J. 14 (2004) 323-329. doi:10.1016/j.idairyj.2003.09.008.

[207] Y.Y. Zhao, F.H. Cao, X.J. Li, D.D. Mu, X.Y. Zhong, S.T. Jiang, Z. Zheng, S.Z. Luo, Effects of different salts on the gelation behaviour and mechanical properties of citric acid-induced tofu, Int. J. Food Sci. Technol. 55 (2020) 785-794. doi:10.1111/ijfs.14348.

[208] X. Li, L. Chen, Y. Hua, Y. Chen, X. Kong, C. Zhang, Effect of preheating-induced denaturation during protein production on the structure and gelling properties of soybean proteins, Food Hydrocoll. $105 \quad$ (2020) 105846. doi:10.1016/j.foodhyd.2020.105846.

[209] E. Ringgenberg, M. Alexander, M. Corredig, Effect of concentration and incubation temperature on the acid induced aggregation of soymilk, Food Hydrocoll. 30 (2013) 463-469. doi:10.1016/j.foodhyd.2012.05.011.

[210] A.H. Clark, S.B. Ross-Murphy, Structural and mechanical properties of biopolymer gels, Biopolymers. (2005) 57-192. doi:10.1007/bfb0023332.

[211] Y. dan Zhu, D. Li, L. jun Wang, Dynamic rheological properties of peanut protein isolate and aggregation suspension and acid-induced gel, Powder Technol. 358 (2019) 95-102. doi:10.1016/j.powtec.2018.08.052.

[212] A.C. Alting, R.J. Hamer, C.G. De Kruif, R.W. Visschers, Cold-set globular protein gels: Interactions, structure and rheology as a function of protein concentration, $\mathrm{J}$. Agric. Food Chem. 51 (2003) 3150-3156. doi:10.1021/jf0209342.

[213] V. Urbonaite, H.H.J. de Jongh, E. van der Linden, L. Pouvreau, Water holding of soy protein gels is set by coarseness, modulated by calcium binding, rather than gel stiffness, Food Hydrocoll. 46 (2015) 103-111. doi:10.1016/j.foodhyd.2014.12.010.

[214] L. Rabiey, M. Britten, Effect of whey protein enzymatic hydrolysis on the rheological 
properties of acid-induced gels, Food Hydrocoll. 23 (2009) 2302-2308. doi:10.1016/j.foodhyd.2009.06.011.

[215] A.C. Alting, R.J. Hamer, C.G. De Kruif, M. Paques, R.W. Visschers, Number of thiol groups rather than the size of the aggregates determines the hardness of cold set whey protein gels, Food Hydrocoll. 17 (2003) 469-479. doi:10.1016/S0268005X(03)00023-7.

[216] T. Aoyama, K. Fukui, T. Nakamori, Y. Hashimoto, T. Yamamoto, K. Takamatsu, M. Sugano, Effect of soy and milk whey protein isolates and their hydrolysates on weight reduction in genetically obese mice, Biosci. Biotechnol. Biochem. 64 (2000) 2594-2600. doi:10.1271/bbb.64.2594.

[217] A.C. Alves, L. Martha, F. Casanova, G.M. Tavares, Structural and foaming properties of whey and soy protein isolates in mixed systems before and after heat treatment, Food Sci. Technol. Int. (2021) 1-9. doi:10.1177/10820132211031756.

[218] S. Joint FAO/WHO/UNU Expert Consultation on Protein and Amino Acid Requirements in Human Nutrition (2002: Geneva, F. and A.O. of the U. Nations, W.H. Organization, U.N. University, Protein and amino acid requirements in human nutrition: report of a joint FAO/WHO/UNU expert consultation, (2007). https://apps.who.int/iris/handle/10665/43411.

[219] S. Comfort, N.K. Howell, Gelation properties of soya and whey protein isolate mixtures, Food Hydrocoll. 16 (2002) 661-672. doi:10.1016/S0268-005X(02)000334.

[220] J. Jose, L. Pouvreau, A.H. Martin, Mixing whey and soy proteins: Consequences for the gel mechanical response and water holding, Food Hydrocoll. 60 (2016) 216224. doi:10.1016/j.foodhyd.2016.03.031.

[221] J.V.C. Silva, R. Cochereau, C. Schmitt, C. Chassenieux, T. Nicolai, Heat-induced gelation of mixtures of micellar caseins and plant proteins in aqueous solution, Food Res. Int. 116 (2019) 1135-1143. doi:10.1016/j.foodres.2018.09.058.

[222] M. Zhang, J. Li, Y. Su, C. Chang, X. Li, Y. Yang, L. Gu, Preparation and characterization of hen egg proteins-soybean protein isolate composite gels, Food Hydrocoll. 97 (2019) 105191. doi:10.1016/j.foodhyd.2019.105191.

[223] D. An, L. Li, The Effect of Limited Proteolysis by Trypsin on the Formation of Soy Protein Isolate Nanofibrils, J. Chem. 2020 (2020). doi:10.1155/2020/8185037. 
[224] Y. Hou, X.H. Zhao, Limited hydrolysis of two soybean protein products with trypsin or neutrase and the impacts on their solubility, gelation and fat absorption capacity, Biotechnology. 10 (2011) 190-196. doi:10.3923/biotech.2011.190.196.

[225] G. Zhao, Y. Liu, M. Zhao, J. Ren, B. Yang, Enzymatic hydrolysis and their effects on conformational and functional properties of peanut protein isolate, Food Chem. 127 (2011) 1438-1443. doi:10.1016/j.foodchem.2011.01.046.

[226] H. AOKI, O. TANEYAMA, M. INAMI, EMULSIFYING PROPERTIES OF SOY PROTEIN: CHARACTERISTICS OF 7s AND IIS PROTEINS, J. Food Sci. 45 (1980) 534-538. doi:10.1111/j.1365-2621.1980.tb04095.x.

[227] H. Hu, E.C.Y. Li-Chan, L. Wan, M. Tian, S. Pan, The effect of high intensity ultrasonic pre-treatment on the properties of soybean protein isolate gel induced by calcium sulfate, Food Hydrocoll. 32 (2013) 303-311. doi:10.1016/j.foodhyd.2013.01.016.

[228] H. Hu, X. Fan, Z. Zhou, X. Xu, G. Fan, L. Wang, X. Huang, S. Pan, L. Zhu, Acidinduced gelation behavior of soybean protein isolate with high intensity ultrasonic pre-treatments, Ultrason. Sonochem. $20 \quad$ (2013) 187-195. doi:10.1016/j.ultsonch.2012.07.011.

[229] P. Zhang, T. Hu, S. Feng, Q. Xu, T. Zheng, M. Zhou, X. Chu, X. Huang, X. Lu, S. Pan, E.C.Y. Li-Chan, H. Hu, Effect of high intensity ultrasound on transglutaminasecatalyzed soy protein isolate cold set gel, Ultrason. Sonochem. 29 (2016) 380-387. doi:10.1016/j.ultsonch.2015.10.014.

[230] H. Hu, X. Zhu, T. Hu, I.W.Y. Cheung, S. Pan, E.C.Y. Li-Chan, Effect of ultrasound pre-treatment on formation of transglutaminase-catalysed soy protein hydrogel as a riboflavin vehicle for functional foods, J. Funct. Foods. 19 (2015) 182-193. doi:10.1016/j.jff.2015.09.023.

[231] H.F. Lin, C.P. Lu, J.F. Hsieh, M.I. Kuo, Effect of ultrasonic treatment on the rheological property and microstructure of tofu made from different soybean cultivars, Innov. Food Sci. Emerg. Technol. 37 (2016) 98-105. doi:10.1016/j.ifset.2016.08.013.

[232] C.S. Paglarini, S. Martini, M.A.R. Pollonio, Physical properties of emulsion gels formulated with sonicated soy protein isolate, Int. J. Food Sci. Technol. 54 (2019) 451-459. doi:10.1111/ijfs.13957.

[233] F. Zhao, X. Liu, X. Ding, H. Dong, W. Wang, Effects of high-intensity ultrasound pretreatment on structure, properties, and enzymolysis of soy protein isolate, 
Molecules. 24 (2019). doi:10.3390/molecules24203637.

[234] G. Zhou, J. Liu, G. Wang, L. Wang, A. Zhang, Y. Wang, X. Wang, Effect of ultrasonic treatment on freeze-thaw stability of soy protein isolate gel, J. Oleo Sci. 68 (2019) 1113-1123. doi:10.5650/jos.ess19167.

[235] A.B. Khatkar, A. Kaur, S.K. Khatkar, Restructuring of soy protein employing ultrasound: Effect on hydration, gelation, thermal, in-vitro protein digestibility and structural attributes, Lwt. 132 (2020). doi:10.1016/j.Iwt.2020.109781.

[236] R. Tian, J. Feng, G. Huang, B. Tian, Y. Zhang, L. Jiang, X. Sui, Ultrasound driven conformational and physicochemical changes of soy protein hydrolysates, Ultrason. Sonochem. 68 (2020) 105202. doi:10.1016/j.ultsonch.2020.105202.

[237] Y. Wang, Z. Wang, C.L. Handa, J. Xu, Effects of ultrasound pre-treatment on the structure of $\beta$-conglycinin and glycinin and the antioxidant activity of their hydrolysates, Food Chem. $218 \quad$ (2017) 165-172. doi:10.1016/j.foodchem.2016.09.069.

[238] T.H. McCann, L. Guyon, P. Fischer, L. Day, Rheological properties and microstructure of soy-whey protein, Food Hydrocoll. 82 (2018) 434-441. doi: 10.1016/j.foodhyd.2018.04.023.

[239] C. Lin, A. Hill, M. Corredig, Gelation of mixtures of soymilk and reconstituted skim milk subjected to combined acid and rennet, J. Texture Stud. 43 (2012) 468-476. doi:10.1111/j.1745-4603.2012.00357.x.

[240] A. Grygorczyk, L. Duizer, I. Lesschaeve, M. Corredig, Gelation of recombined soymilk and cow's milk gels: Effect ofhomogenization order and mode of gelation on microstructure andtexture of the final matrix, Food Hydrocoll. 35 (2014) 69-77. doi:10.1016/j.foodhyd.2013.04.011.

[241] A. Grygorczyk, M. Alexander, M. Corredig, Combined acid- and rennet-induced gelation of a mixed soya milk-cow's milk system, Int. J. Food Sci. Technol. 48 (2013) 2306-2314. doi:10.1111/ijfs.12218.

[242] R.R. Roesch, M. Corredig, Heat-induced soy-whey proteins interactions: Formation of soluble and insoluble protein complexes, J. Agric. Food Chem. 53 (2005) 34763482. doi:10.1021/jf048870d.

[243] S.N. Anuradha, V. Prakash, Complexation of bovine $\beta$-lactoglobulin with $11 \mathrm{~S}$ protein fractions of soybean (Glycine max) and sesame (Sesamum indicum), Int. J. Food 
Sci. Nutr. 60 (2009) 27-42. doi:10.1080/09637480701877736.

[244] C.M. Beliciu, C.I. Moraru, The effect of protein concentration and heat treatment temperature on micellar casein-soy protein mixtures, Food Hydrocoll. 25 (2011) 1448-1460. doi:10.1016/j.foodhyd.2011.01.011.

[245] M.H. Alu'Datt, I. Alli, M. Nagadi, Preparation, characterization and properties of whey-soy proteins co-precipitates, Food Chem. 134 (2012) 294-300. doi:10.1016/j.foodchem.2012.02.142.

[246] R. Ingrassia, J.P. Costa, M.E. Hidalgo, M. Mancilla Canales, H. Castellini, B. Riquelme, P. Risso, Application of a digital image procedure to evaluate microstructure of caseinate and soy protein acid gels, LWT - Food Sci. Technol. 53 (2013) 120-127. doi:10.1016/j.Iwt.2013.01.021.

[247] C.M. Beliciu, C.I. Moraru, Physico-chemical changes in heat treated micellar casein - Soy protein mixtures, LWT - Food Sci. Technol. 54 (2013) 469-476. doi:10.1016/j.Iwt.2013.06.013.

[248] Y. Su, Y. Dong, F. Niu, C. Wang, Y. Liu, Y. Yang, Study on the gel properties and secondary structure of soybean protein isolate/egg white composite gels, Eur. Food Res. Technol. 240 (2015) 367-378. doi:10.1007/s00217-014-2336-3.

[249] A.M. Oechsle, M. Häupler, M. Gibis, R. Kohlus, J. Weiss, Modulation of the rheological properties and microstructure ofcollagen by addition of co-gelling proteins, Food Hydrocoll. 49 (2015) 118-126. doi:10.1016/j.foodhyd.2015.03.013.

[250] H. Jian, F. Qiao, P. Yang, F. Guo, X. Huang, B. Adhikari, J. Chen, Roles of soluble and insoluble aggregates induced by soy protein processing in the gelation of myofibrillar protein, Int. J. Food Sci. Technol. 51 (2016) 480-489. doi:10.1111/ijfs.12992.

[251] A.M. Oechsle, M. Häupler, F. Weigel, M. Gibis, R. Kohlus, J. Weiss, Modulation of extruded collagen films by the addition of co-gelling proteins, J. Food Eng. 171 (2016) 164-173. doi:10.1016/j.jfoodeng.2015.10.004.

[252] C. Ersch, E. van der Linden, P. Venema, A. Martin, The microstructure and rheology of homogeneous and phase separated gelatine gels, Food Hydrocoll. 61 (2016) 311317. doi:10.1016/j.foodhyd.2016.05.022.

[253] J.V.C. Silva, G. Balakrishnan, C. Schmitt, C. Chassenieux, T. Nicolai, Heat-induced gelation of aqueous micellar casein suspensions as affected by globular protein 
addition, Food Hydrocoll. 82 (2018) 258-267. doi:10.1016/j.foodhyd.2018.04.002.

[254] D. Lin, L. Zhang, R. Li, B. Zheng, M.C. Rea, S. Miao, Effect of plant protein mixtures on the microstructure and rheological properties of myofibrillar protein gel derived from red sea bream (Pagrosomus major), Food Hydrocoll. 96 (2019) 537-545. doi:10.1016/j.foodhyd.2019.05.043.

[255] C. Wu, X. Yan, T. Wang, W. Ma, X. Xu, M. Du, A self-sorted gel network formed by heating a mixture of soy and cod proteins, Food Funct. 10 (2019) 5140-5151. doi:10.1039/c9fo00560a.

[256] Z. Pang, Y. Luo, B. Li, M. Zhang, X. Liu, Effect of different hydrocolloids on tribological and rheological behaviors of soymilk gels, Food Hydrocoll. 101 (2020) 105558. doi:10.1016/j.foodhyd.2019.105558.

[257] C.D. Munialo, E. van der Linden, H.H.J. de Jongh, The ability to store energy in pea protein gels is set by network dimensions smaller than 50nm, Food Res. Int. 64 (2014) 482-491. doi:10.1016/j.foodres.2014.07.038.

[258] M. Stading, M. Langton, A. Hermansson, Small and large deformation studies of protein gels, J. Rheol. (N. Y. N. Y). 39 (1995) 1445-1450. doi:10.1122/1.550646. 


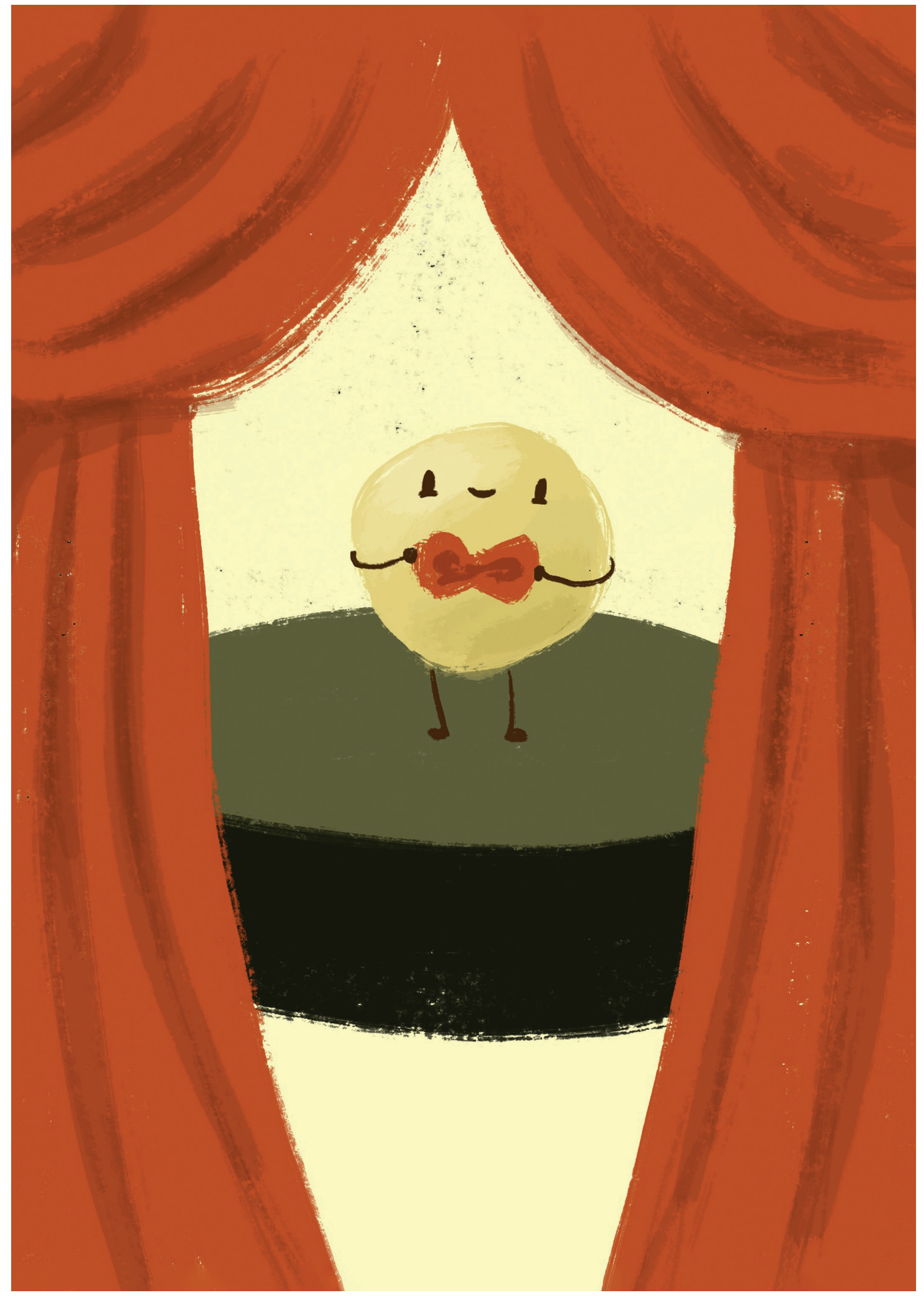




\section{Acknowledgement}

A Saturday morning in the winter, the swans are peacefully sitting beside the lake in front of the Forum, the campus is silent and beautiful, exactly like the day when I came here. Four years ago, I left my hometown Wuhan, a place where I had lived for 25 years, to Wageningen, for my PhD dream. Time flies, now this journey is about to end. It was an amazing journey, full of laughter and tears, full of success and regrets. Most importantly, I have gained a lot of friends, teachers, and mentors during this journey. Without their supports and help, I could not have made it.

First of all, I want to thank my supervisors, Leonard Sagis and Siyi Pan. Leonard, thank you for giving me the opportunity to study at this prestigious university. Your guidance helped me to unlock the door of the rheology world. Now, I know all matters are deforming and flowing in a way that can be explained. Your "freedom" allowed me to become an independent researcher and critical thinker. Now, I know how to solve scientific problems on my own. Most importantly, I appreciate your trust and encouragement in these years. I remember your trust when I tried to improve my oral English; I also remember your "high five" after my presentation in Zurich. All of these scenes will continually inspire me for life! 潘老师, 同样谢谢您。谢谢你接受我作为你的硕士, 后来又支持我来荷兰求学。真的也很庆幸能作为您的 学生。您的学识和成就是我努力的方向, 您的阅历和见解为我的发展指明了道路。您总是能为学生着想, 让我按自己设计的方向好好努力, 无需顾虑其他, 至今学生还深深感动和感激着!

Next, I would like to thank all the teaching staff at the Food Physics group. Erik, although you are not my direct supervisor, I am glad to have a "fibril bond" with you. I sincerely appreciate your help and guidance on my career development. I will also keep your words in mind: do meaningful research and do not make "noise" to the science field. Elke, the teacher of the year, thank you for organizing so many group activities for us, especially the Christmas dinner that year, I felt the warmth of home that night. Paul, Mehdi, Guido, Jasper, thanks for kindly answering all my questions in the last four years. Your expertise and wisdom inspired me a lot. I also want to thank all the technicians in our group: Harry, Miranda, Roy, Floris, David. Without your efforts, I could not perform my experiments in such organized and well-functioned laboratories. Thank you for helping me order reagents and teaching me how to use experimental apparatus. Of course, I am also very indebted to our secretary, Els. Thanks for arranging so many things for me: from a studio at the beginning to a defence date at the end. Your help makes my life in Wageningen much easier.

Finally, I would like to thank all my PhD fellows and students who accompanied me on this journey. First, my "hot pot" officemates (Remco, Xilong, Naomi), and my "hot-pot chicken" officemates (Belinda, Philipp, Evelen, Thiemo, Anteun, Umer). Thank you for all the 
office talks, jokes, and meals. I will miss those joyful moments. Then, my "brother" Lei, my "aunt", Claudine, my "weight buddies" Jack \& Remco, my "HZAU buddies" PDF \& Cai, my "basketball coach" Xiangyu, my "cheerleader" Qi, my "Chocolate queen" Annika, my "pajamas queen" Ninna, and my friends from all over the world: "amigo" Gerard, "amico" Marco, "دوست" Melika, Aref, Parisa, "เพื่อน" Ornicha, "友達" Naoya. I really enjoyed the time we spent together, and I have also learned a lot from you. Also, Zhihong, Xudong, Bo, Penghui, Xiao, Pauline, Raisa, Suraj, Ahmed, Momo, hongwei, zongyao, Wangli, jilu, wanqing, sicong, dazhi, peicheng, Liukun, pingping, Yuena, miaomiao, yifan, Caohui, Liuyan, shanshan, yanjun, xiaofei, Zhuzhe, etc. Thank you for all the nice chats inside and outside of work. And also, the students I supervised: Madelon, Wingkie, Celia, Tjitske, Linfeng, Moyan. Thanks for your contribution to this thesis, especially Wingkie, I am very happy that we have developed our cooperation into friendship. Finally, I would particularly like to thank Huifang Cai (cool Cai, beer queen) and Qiuhuizi (Evelen, mungbean queen) for being my Paranymphs and preparing the big party. Cai, I wish that happy angel can stay in your heart forever. Evelen, I wish your Mr. Right will find you soon.

好了, 响起那首《楼上来的声音》吧, 这是属于我们的歌。哲哥, 谢谢四年的照顾, 我会怀念我们一起 “勤劳致富” 的日子, 这段友谊和经历是我一生的财富。希望以后你能和你家舟妹过上幸福团圆的日子。 蒋爹, 谢谢四年的扶持, 是你开启了我厨艺的新境界, 从此我的 “幸福指数” 和体重大幅度提高。我相信 生活也能对你温柔以待。还有登科, 桃军, 锡龙, 虚船, 谢谢我们一起聚餐吹牛, 骑车放松, 旅行看鲸的 日子。然后, 茄妹。谢谢你多年的陪伴和守候。很庆幸和骄傲我们能够克服重重困难, 不离不弃。感谢你 包容我写论文时的各种少爷病, 替我做饭, 帮忙购物。希望你的博士之路一帆风顺, 也希望我们今后的生 活都够一直幸福。也谢谢我在国内的老师和伙伴们, 谢笔钧老师, 徐晓云老师, 刘风霞老师, 黄行健师 兄, 田明师兄, 燕姐, 源源, 老张, Faru, 海娟, 珊珊, 丹郡, A 哥, 谢谢你们的支持和关心。

最后, 我要谢谢我的家人。首先是我的父母, 夏警官和许会计, 谢谢你们多年来的含辛茹苦和默默付出, 为我创造了一个良好成长环境, 并为我树立了为人处世的榜样。老妈总是说 “有我真好” , 其实我想说, “有你们才真好! ” 其次, 我想谢谢姨妈, 漺夫, 小贯, 还有外婆, 谢谢你们从小到大, 在我上学路上给 我做的后勤工作, 尤其是外婆, 高三那年你冒着大雪给我送的饭是我人生最难忘的一餐。最后, 在 2020 年相继离世的外公和伯, 谢谢你们从小到大的照顾, 原凉我没能回去送你们最后一程, 愿天堂再无伤痛。

Very heavily I take my leave, as heavily as I came here. I came with dreams full of my heart, and I leave with memories full of my mind. (我没有轻轻的走, 正如我没有轻轻的来。我来时揣着 满怀的梦想, 我走时带着无限的回忆) 。 


\section{About the author}

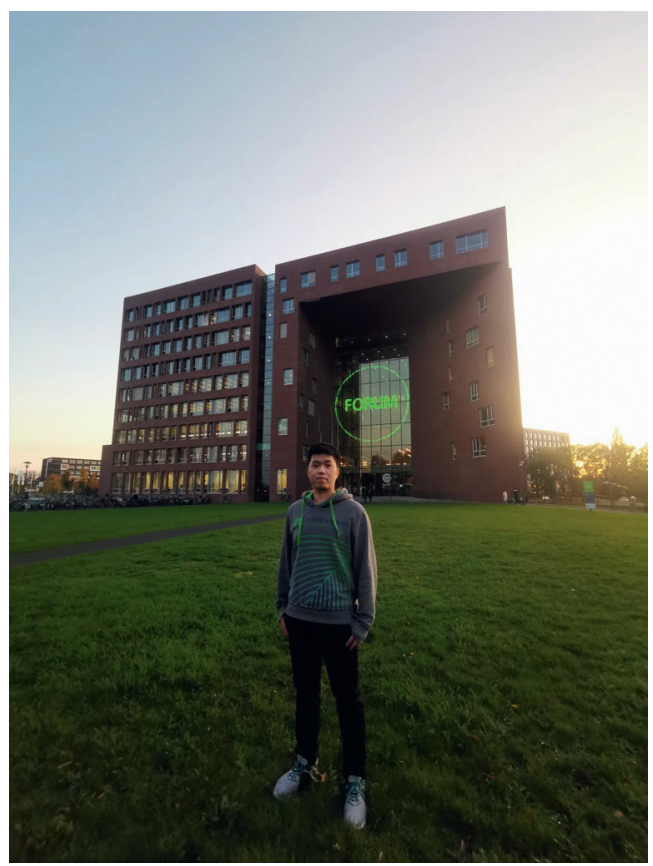

Wenjie Xia was born on July 16, 1992 in Wuhan city, China. After he obtained his master's degree, with the subject "Food Science and Technology", at Huazhong Agricultural University in Wuhan. His interest in food science, especially plant-based proteins, brought him to Wageningen University in 2018 to pursue a doctoral degree. During his PhD, he worked on the effects of different protein modifications on the aggregation and gelation of soy protein. The results of his $\mathrm{PhD}$ project are presented in this thesis. After his PhD, Wenjie Xia will continue working as a researcher in the field of food science.

Wenjie Xia: 411951439@qq.com

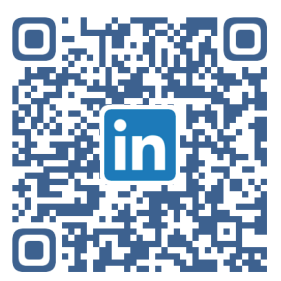




\section{List of publications}

\section{This thesis}

W. Xia, H. Zhang, J. Chen, H. Hu, F. Rasulov, D. Bi, X. Huang, S. Pan, Formation of amyloid fibrils from soy protein hydrolysate: Effects of selective proteolysis on $\beta$-conglycinin, Food Res. Int. 100 (2017) 268-276. doi: 10.1016/j.foodres.2017.08.059.

W. Xia, S. Pan, Z. Cheng, Y. Tian, X. Huang, High-intensity ultrasound treatment on soy protein after selectively proteolyzing glycinin component: Physical, structural, and aggregation properties, Foods. 9 (2020) 839. doi: 10.3390/foods9060839.

W. Xia, W.K. Siu, L.M.C. Sagis, Linear and non-linear rheology of heat-set soy protein gels: Effects of selective proteolysis of $\beta$-conglycinin and glycinin, Food Hydrocoll. 120 (2021) 106962. doi: 10.1016/j.foodhyd.2021.106962.

W. Xia, L Zhu, R. Delahaije, Z Cheng, X Zhou, L.M.C. Sagis. Acid-induced gels from soy and whey protein thermally-induced mixed aggregates: Rheology and microstructure. (Accepted)

\section{Other works}

J. Yang*, W. Xia*, T. Botma, L.M.C. Sagis. Selective proteolysis of $\beta$-conglycinin as a tool to increase air-water interface and foam stabilising properties of soy proteins. (In preparation)

W. Xia*, Q. Yang*, M. Zhang, L.M.C Sagis, P Venema. Linear and non-linear rheological properties of mung bean protein gels: effects of mild processing (In preparation)

D. Xie, X. Liu, H. Zhang, W. Xia, X. Huang, D. Bi, S. Pan, Textural properties and morphology of soy $7 \mathrm{~S}$ globulin-corn starch (amylose, amylopectin), Int. J. Food Prop. 20 (2017) 2197-2205. doi:10.1080/10942912.2016.1233430.

Shared first authorship: * 


\section{Overview of completed training activities}

\section{Discipline specific courses}

Food proteins: functionality, modifications and analysis (7th edition) ${ }^{1}$

Advanced food analysis

VLAG, the Netherlands

Rheology short courses

VLAG, the Netherlands

Rheology course

Online

VLAG, the Netherlands

\section{Conferences and Symposia}

11th microencapsulation training school ${ }^{1}$

Loughborough, United

$8^{\text {th }}$ ISFRS conference ${ }^{1}$

Kingdom

Zurich, Switzerland

18th International congress on rheology

Online

2020

Science and Technology for Meat

Online

Analogues

$35^{\text {th }}$ EFFoST conference ${ }^{2}$

Lausanne, Switzerland

$1^{\text {st }}$ International conference on frontier technology of food science ${ }^{1}$

Online

\section{General courses}

VLAG PhD week

Brain Training

Effective behaviour in your professional surroundings

Searching and Organising Literature

Scientific writing

Writing propositions for your PhD

Last Stretch of the PhD Programme

Career Perspectives

Mobilising your scientific network

\section{Other activities}

Preparation of research proposal

Weekly group meetings at $\mathrm{FPH}^{1}$

\footnotetext{
${ }^{1}$ Oral presentation, ${ }^{2}$ poster presentation
} 


\section{Colophon}

This research described in this thesis was supported by the laboratory of Physics and Physical Chemistry of Foods (FPH), Wageningen University \& Research, The Netherlands, and also the State Key Laboratory of Environment Correlative Dietology, Huazhong Agricultural University, China.

The financially support from China Scholarship Council (CSC) and Wageningen University \& Research for performing this research and for printing this thesis is gratefully acknowledged.

Cover design: Moyan Zhang, Wenjie Xia

Thesis layout: Wenjie Xia

Printed by: ProefschriftMaken

Copyright (c) Wenjie Xia, November 2021 